\title{
LA-6654-MS
}

Informal Report

Special Distribution

Issued: November 1977

\section{Uranium Hydrogeochemical and Stream Sediment Reconnaissance of the Northern Part of the Powder River Basin, Wyoming}

by

Wayne A. Morris 
Th1s work was supported by the US Department of Energy,

Division of Uranium Resources and Enrichment. Program Code BO48.

This report was prepared as an account of work sponsored by the United States Government. Neither the United States nor the United States Department of Energy, nor any of their employees, nor any of their contractors, subcontractors, or their employees, makes any warranty, express or implied, or assumes any legal liability or responsibility for the accuracy, completeness, or usefulness of any information, apparatus, product, or process disclosed, or represents that 1 ts use would not infringe privately owned rights. 


\section{CONTENTS}

LIST OF ILLUSTRATIONS iv

LIST OF TABLES iv

ABSTRACT 1

I. INTRODUCTION 2

II. DESCRIPTION OF STUDY AREA

$\begin{array}{lr}\text { Location } & 2 \\ \text { Geographic Setting } & 2 \\ \text { Climate } & 4 \\ \text { Hydrology } & 6 \\ \text { Geology } & 6\end{array}$

III. REPORTED URANIUM OCCURRENCES IN THE STUDY AREA 8

IV. STATISTICAL DATA AND EVALUATION OF URANIUM

CONCENTRATIONS IN WATER 10

V. STATISTICAL DATA AND EVALUATION OF URANIUM
CONCENTRATIONS IN SEDIMENT

VI. CONCLUSIONS 18

$\begin{array}{ll}\text { ACKNOWLEDGMENTS } & 19\end{array}$

REFERENCES CITED $\quad 20$

APPENDIX A - Listings of Field Data and Uranium Concentrations for Water Samples 23

A-I - Water Samples Analyzed by Fluorometry 24

A-II - Water Samples Analyzed by Delayed-Neutron Counting 44

APPENDIX B - Listings of Field Data and Uranium Concentrations for Sediment Sampies 49

APPENDIX C - Explanation of Codes Used in the LASL HSSR Data Listings 69

C-I - Code for LASL HSSK Open-File Data Listings 70

C-II - Numerical Key and Specifications for Sample Types Taken by the LASL

APPENDIX D - Summary of Standard Procedures Used by the LASL in the US ERDA NURE HSSR 77 


\section{LIST OF ILLUSTRATIONS}

Fig. No.

1. Powder River Basin study area, northeast Wyoming.

2. Major structures of the Powder River Basin, showing known uranium districts.

3. East-west section across the Powder River Basin. 7

4. Geologic units of the Powder River Basin. 9

5a. Distribution of uranium concentrations in water samples.

5b. Distribution of uranium concentrations in sediment samples.

Plate No.

I. Geologic map.

In pocket

II. Overlay showing numbered sample locations.

in pocket

III. Overlay showing uranium concentrations in waters.

in pocket

IV. Overlay showing uranium concentrations in sediments. in pocket

\section{LIST OF TABLES}

Table No.

I. Reported Uranium Occurrences in the Powder River Basin Study Area

II. Uranium Concentrations by Type of Water Sample

III. Uranium Concentrations by Ranges for Waters

IV. Uranium Occurrences Reflected in Water Results

16

v. Uranium Concentrations by Type of Sediment Sample

VI. Urantum Concentrations by Ranges for Sediments 


\section{URANIUM HYDROGEOCHEMICAL AND STREAM SEDIMENT RECONNAISSANCE}

OF THE NORTHERN PART

OF THE POWDER RIVER BASIN, WYOMING

by

Wayne A. Morris

\section{ABSTRACT}

During the spring and summer of 1976,2144 water and watertransported sediment samples were collected from 1685 locations spread over an approximate $21000 \mathrm{~km}^{2}$ area of the northern end of the Powder River Basin in northeast Wyoming. All of the samples were analyzed for total uranium at the LASL, using standardized procedures and rigorous quality controls--the waters by fluorometry and the sediments (and those waters with $>10 \mathrm{ppb}$ uranium) by delayedneutron counting. The 1178 water samples were collected from 111 streams, 19 natural ponds, 142 springs, 186 artificial ponds, and 720 wells. The 966 sediment samples were collected from 108 active streams, 415 dry streams, 20 wet natural ponds, 9 dry natural ponds, 131 flowing springs, 12 dry springs, 170 wet artificial ponds, and 101 dry artificial ponds. All of the field collection, treatment, and packaging of the samples was performed following strict LASL specifications. The uranium concentrations measured in the waters range from undetectable $(<0.2 \mathrm{ppb})$ to $295.8 \mathrm{ppb}$ and have a mean value of $7.3 \mathrm{ppb}$. The high uranium concentrations in the waters of this area are thought to be due primarily to a general abundance of readily soluble uranium in the Wasatch and Fort Union formations throughout the Powder River Basin. Those locations which have abnormally high uranium were examined more closely, and follow-up field examinations are recommended in the vicinity of some of these sites. The uranium content of the sediment samples ranges from 0.5 to $11.2 \mathrm{ppm}$ and has a mean value of $3.4 \mathrm{ppm}$. Sample locations with anonalous uranium values were examined with respect to the local geology and other relevant factors, and an area believed to be favorable for a follow-up field investigation is indicated. A distinct correlation between the high uranium in waters and sediments and the Wasatch and Fort Union formations, which are associated with nearby producing uranium districts, is evident at several locations. 


\section{INTRODUCTION}

This report describes work done by the Los Alamos Scientific Laboratory (LASL) as part of the nationwide Hydrogeochemical and Stream Sediment Reconnaissance (HSSR) for uranium. The work is part of the National Uranium Resource Evaluation (NURE) program, being sponsored by the US Energy Research and Development Administration (ERDA), to determine whether the potential uranium resources of the US will meet future needs for nuclear power generation. The HSSR is designed to identify areas having higher than normal concentrations of uranium in ground waters, surface waters, and water-transported sediments and to aid private industry in selection of areas for exploration. The region assigned the LASL for the reconnaissance includes the Rocky Mountain states of New Mexico, Colorado, Wyoming, and Montana and the state of Alaska.

\section{DESCRIPTION OF STUDY AREA}

The study area dealt with covers the northern portion of the Powder River Basin in northeast Wyoming (Fig. 1). Water and sediment samples were collected by a commercial contractor from 1620 sample locations between late March and early July 1976. Some 65 additional locations were sampled in September 1976 to fill gaps in the areal coverage. The sample locations were specified by the LASL, and the samples were collected using maps, field equipment, and materials furnished by the LASL. Standardized field and analytical procedures developed by the LASL (Sharp, 1977) were utilized throughout the work so the data of this study could be compared with those of other LASL HSSR reports.

\section{Location}

The study area is shown in relation to surrounding structural features in Fig. 2. The Powder River Basin lies along the eastern flank of the Rocky Mountains in northern Wyoming and southern Montana. In Wyoming, it is bounded on the west by the Bighorn Mountains, on the south by the Hartville uplift, Casper arch, and Laramie Mountains, and on the east by the Black Hills. To the north, it extends into Montana where it is separated by low arches from the Great Plains. The study area, proper, spreads across $21000 \mathrm{~km}^{2}$ of the northern end of the Powder River Basin, lapping onto the eastern flank of the Bighorn Mountains. It is bounded by $107^{\circ} 7.5^{\circ} \mathrm{W}$ longitude on the west, $105^{\circ} \mathrm{W}$ longitude on the east, $44^{\circ} \mathrm{N}$ latitude on the south (between $105^{\circ}$ and $106^{\circ} 30^{\circ}$ W longitude), and $45^{\circ} \mathrm{N}$ latitude on the north (Montana state line). In the southwest corner of the area, the boundary runs from $44^{\circ} \mathrm{N}$ latitude, $106^{\circ} 30^{\prime}$ $\mathrm{W}$ longitude, southeast to the intersection of $43^{\circ} 30^{\prime} \mathrm{N}$ latitude and $106^{\circ} \mathrm{W}$ longitude, then west along $43^{\circ} 30^{\circ} \mathrm{N}$ latitude to $107^{\circ} 7.5^{\prime} \mathrm{W}$ longitude (Figs. 1 and 2). The irregularity of the southern boundary resulted when the Pumpkin Buttes uranfum mining district was eliminated from this reconnaissance in 1976 at the request of the ERDA.

\section{Geographic Setting}

The area covers parts of three National Topographic Map Series (NTMS) map sheets (1:250 000-scale): the west half of the Gillette sheet, the east half of the Sheridan sheet, and part of the northeast quarter of the Arminto sheet. It is traversed from west to east by I-90 and from south to north by I-25 and has three other main hard-surfaced roads. The area is populated by approximately 35000 people. Sheridan, the largest town in the area, has over 


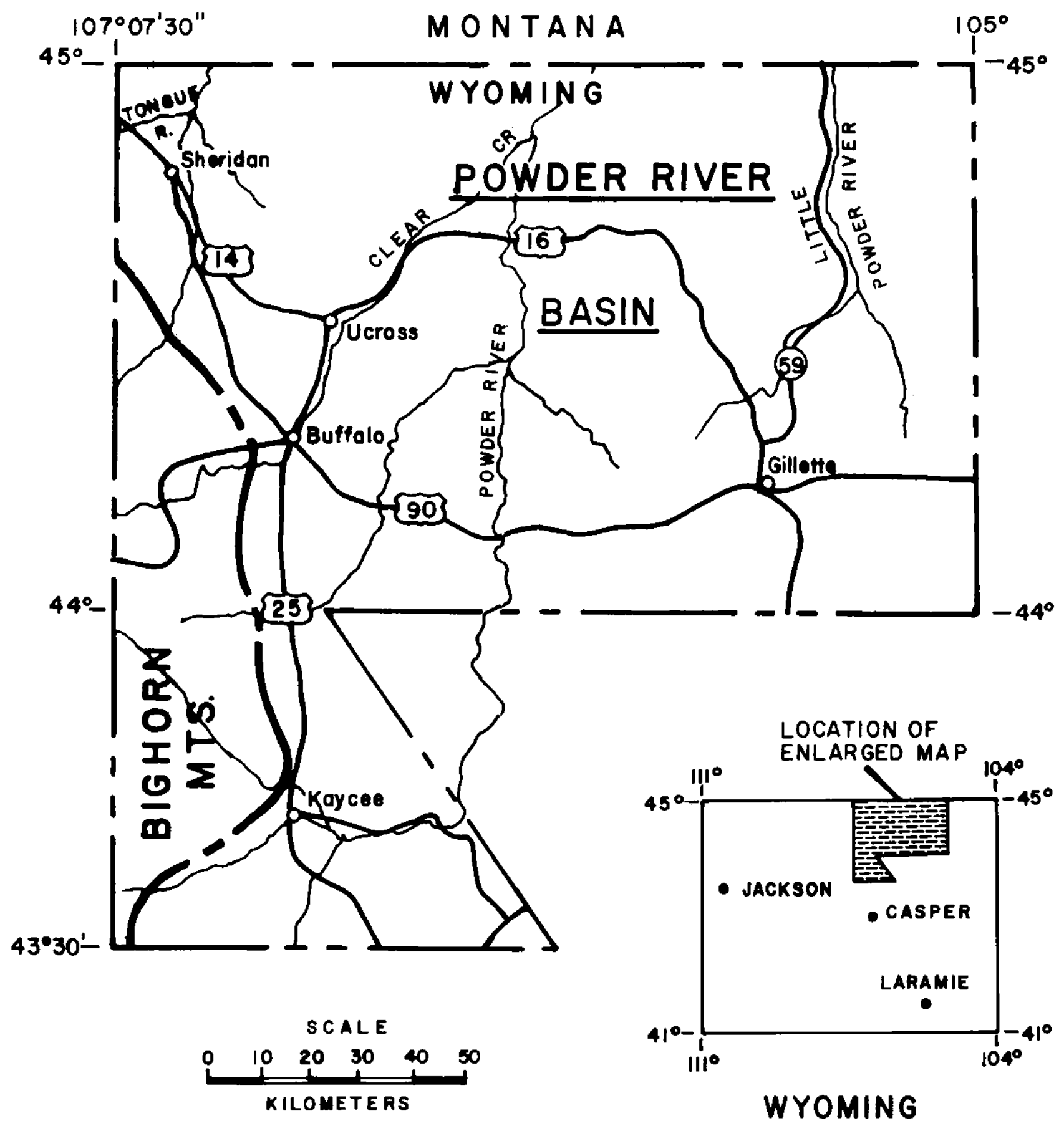

Fig. 1

Powder River Basin study area, northeast Wyoming. 
11000 residents; Gillette, the second largest, has 7300; and Buffalo, 3500 . Gillette is growing rapidly due to the recent expansion of the coal, oil, and gas industries there. The major activities in the area are coal mining, oil and gas production, agriculture, and government. Uranium mining has been an important industry to the east and south of the study area during the last 25 years (Fig. 2). The land ownership is about $68 \%$ private, $25 \%$ federal, and $7 \%$ state.

Elevations in the study area vary from $4030 \mathrm{~m}$ at Cloud Peak in the Bighorn Mountains to $1040 \mathrm{~m}$ at the bottom of the Powder River drainage where it enters Montana. Most of the basin is below $1530 \mathrm{~m}$ elevation, is of low relief, and slopes to the east and north. Vegatation in the basin is principally sagebrush, native grasses, and prickly pear, although dense growths of willows, ash, and cottonwoods flourish along some river valleys. At higher elevations, the slopes and uplands in the Bighorn Mountains support large stands of pine, spruce, and cedar trees. Aspen groves thrive in the mountain meadows. Above the timberline the vegetation is largely restricted to moss, lichens, dwarf willows, and a sparse cover of hardy native grasses in protected areas (Whitcomb et al, 1966).

\section{Climate}

The Powder River Basin has a cool, semiarid climate due to the combination of its relatively high elevation and its loction immediately east of the Bighorn Mountains. The Bighorns, with elevations above $3500 \mathrm{~m}$, strip most of the molsture from the westerly winds that prevall over Hyoming. Also, these westerlies tend to block moist air masses from Canada or the Gulf of Mexico from moving into the basin, particularly in the winter when they are strongest and most predominant. This accounts for the extremely dry winter months which average less than $13 \mathrm{~mm}$ of precipitation. Occasionally, fastmoving winter storms come in from the north and east, causing heavy precipitation when the air is uplifted and cooled as it approaches the mountains. These upslope winds also account for the heaviest amounts of precipitation in the basin during late spring and early summer when the winter westerlies become less predominant and moist air from the Gulf of Mexico circulates into the basin. Thermally-induced thunderistorms account for large amounts of precipitation near the mountains during the sumer months. The annual precipitation in the study area averages between $280 \mathrm{~mm}$ in the central basin to 500-650 $\mathrm{mm}$ in the higher elevations of the Bighorn Mountains. About half of the annual precipitation occurs in late spring and early summer; about a third of the total falls as snow, with the heaviest accumulations in December and early spring.

However, most of the Powder River Basin averages less than $350 \mathrm{~m}$ of precipitation a year and has a generous amount of sunshine the year around. Summer days are hot throughout the basin, with temperatures near $30^{\circ} \mathrm{C}$, but the nights are cool, around $10^{\circ} \mathrm{C}$. Winter temperatures, which average $2-5^{\circ} \mathrm{C}$ for daytime highs and $-12^{\circ} \mathrm{C}$ for nightime lows, are moderated by "Chinook" or downslope winds, which are heated by compression as they descend from higher elevations. Due to the air-mass control of the climate of the basin, large, rapid changes in the temperature can be expected throughout the year. Extreme temperatures of near $38^{\circ} \mathrm{C}$ in summer and $-40^{\circ} \mathrm{C}$ in winter have been recorded. Additional information on the climate of the Powder River Basin is provided by Becker and Alyea (1964a and b).

Except for late May and all of June, which were much wetter than usual, the weather during the sampling period was slightly warmer and wetter than normal. In the eastern part of the study area, the amount of precipitation 

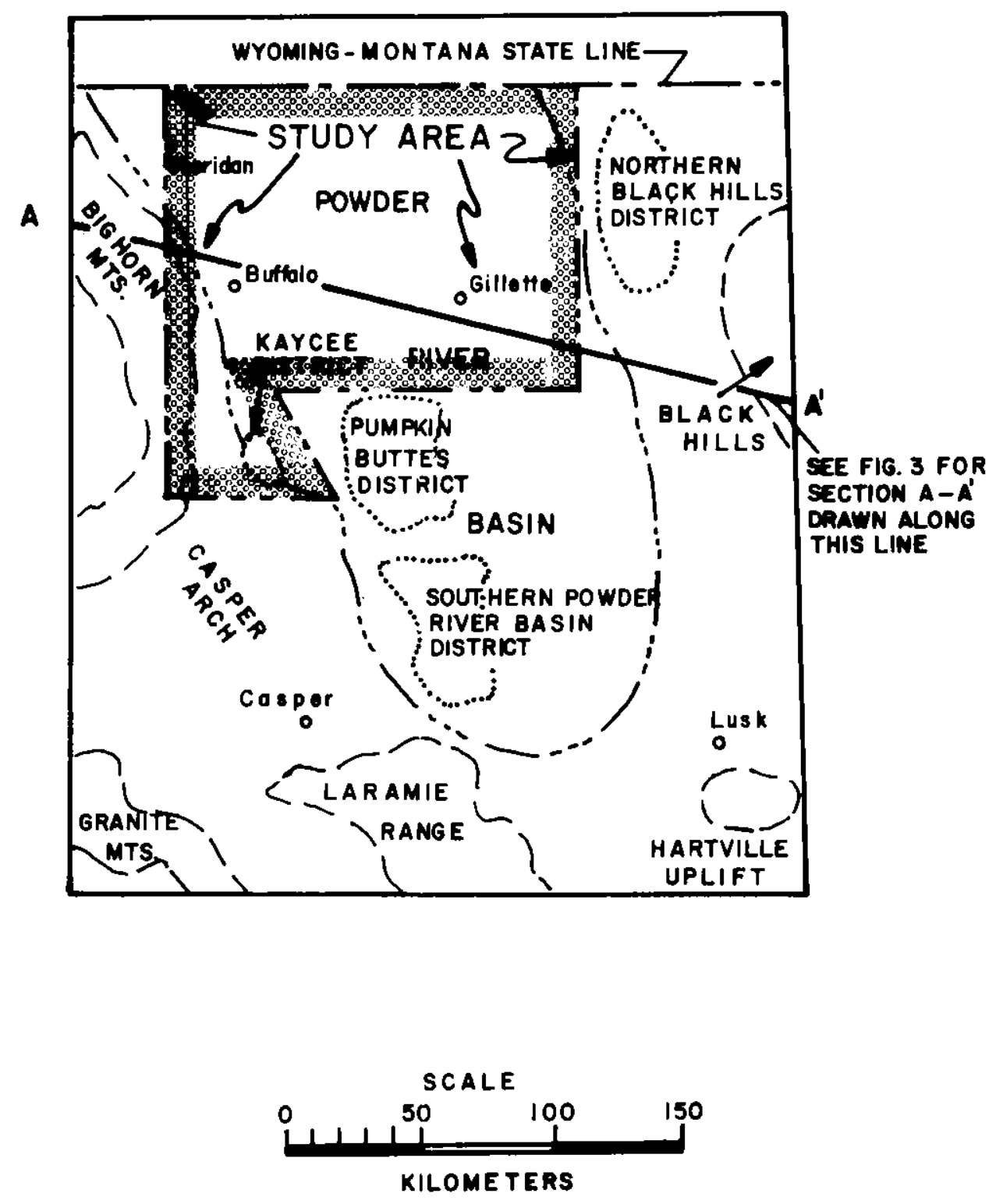

Fig. 2

Major structure of the Powder River Basir, showing known uranium districts. 
during May was about twice the normal average of $50 \mathrm{~mm}$ for that month. During June, the normal average of $100 \mathrm{~mm}$ was exceeded by roughly an equal amount throughout the whole study area. Heavy, basin-wide rains of 25-50 mm fell on May 30 and June 6,14 , and 23 . Some rain (a trace to $15 \mathrm{~mm}$ ) fell almost every day from June 6 to 18 (NOAA, 1976).

\section{Hydrology}

The Powder River Basin study area is drained to the north by the Tongue, Powder, and Little Powder River systems and to the east by the Belle Fouche and Little Missouri River systems. Except for streams in the mountains and the few large rivers, the area has little surface water during much of the year. The evaporation potential in most of the basin is several times higher than the total precipitation. Consequently, except for the surface runoff after storms, much of the surface water, snow, and soil moisture is lost to the atmosphere by evaporation. However, an even balance between the recharge and discharge of the ground water system is indicated by only slight variation in the water levels in observation wells. Recharge of the ground water system is mainly from precipitation in the basin and from precipitation, irrigation, and mountain streamflow near the Bighorn Mountains. Discharge is mainly due to the pumping of wells, evaporation, and transpiration by vegetation.

The predominant use of ground water in the Powder River Basin is for domestic and livestock purposes, although some irrigation wells have been developed from the Madison limestone (Mississippian) along the Bighorn Mountains. The main aquifers throughout most of the basin are the Fort Union and Wasatch formations. The ground waters in these formations are usually close to the surface (within $30 \mathrm{~m}$ ), but wells generally yield less than one liter per second. Flowing wells are abundant, particularly in the north and northeast portion of the basin. Much of the water from both formations is undesirable for domestic use because of the high level of dissolved solids (generally $500-1500 \mathrm{mg} / \ell$, with highs of $8000 \mathrm{mg} / \ell$ ), and some water is unfit for watering stock. The ground water potentials of the Paleozoic and Mesozoic formations, which are deeply buried throughout most of the basin, are largely unknown. Except near the Bighorn Mountains, where the alluvium is thicker and has better recharge, alluvial flood plains along the major rivers of the basin generally do not produce water. Detailed information on the potential water supply of the geologic formations of the Powder River Basin, and on the quantity and quality of both surface and ground water, is provided by Kohout (1957), Lowry and Cummings (1966), Whitcomb et al (1966), Hodson (1971), Hodson et al (1973), and Breckenridge et al (1974), among others.

\section{Geology}

The Powder River Basin is a northwest-trending structural and topographic basin which formed in the Rocky Mountain foreland during the Laramide Orogeny in late Cretaceous-early Tertiary time. It is located in northeast Wyoming and southeast Montana, along the Powder River drainage. In Wyoming, it is surrounded by the Bighorn Mountains on the west, by the Black Hills monocline on the east, and by the Hartville uplift, the Laramie Mountains, and the Casper arch on the south (Fig. 2). It is asymetric, with a steeplydipping western limb ( $\backsim 30$ degrees) and a gently-dipping eastern limb ( $\Omega 3$ to 5 degrees), as shown considerably exaggerated in Fig. 3. The axis of the basin is located west of the Powder River, as shown on Plate I, the generalized geological map of the study area. 


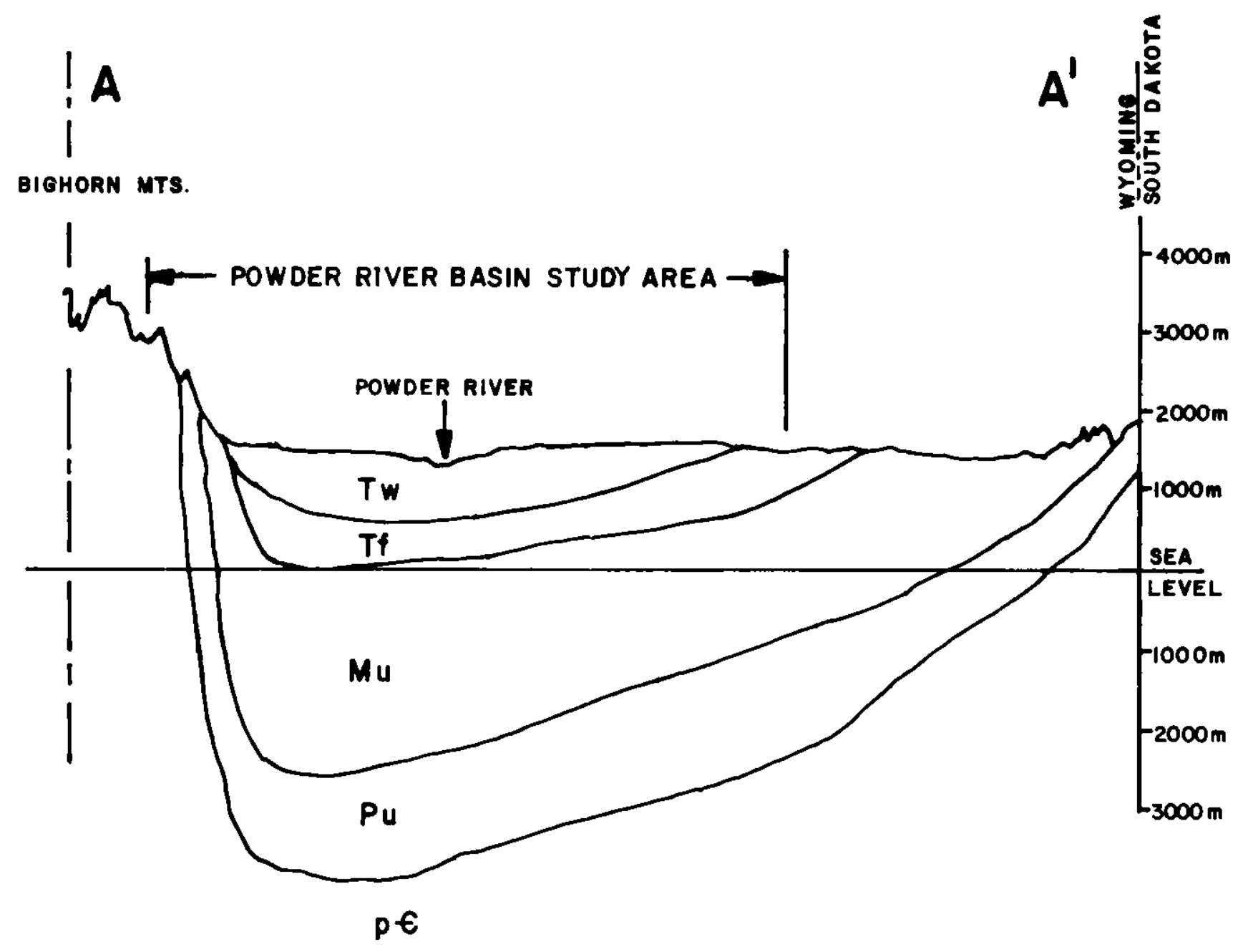

TW - WASATCH FORMATION

Tf - FORT UNION FORMATION

MU-MESOZOIC - UNDIFFERENTIATED

PU-PALEOZOIC - UNDIFFERENTIATED

PE-PRE CAMBRIAN

(AFTER RENFRO AND FERAY, 1975)

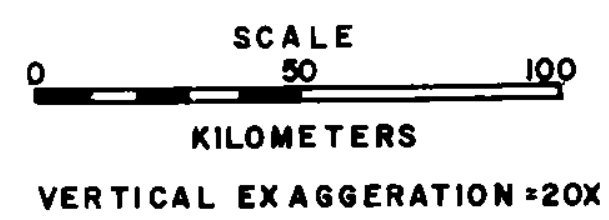

Fig. 3

East-west section across the Powder River Basin. 
The thickest sedimentary section in the basin, comprised of $5500 \mathrm{~m}$ of Cambrian to Tertiary (Eocene) strata, is located alongside the Bighorn Mountains in the southwest corner of the study area. The west edge of the basin section has been sharply folded and faulted along a narrow zone on the eastern flank of the Bighorn Mountains, where near-vertical to overturned Paleozolc and Mesozoic sedimentary rocks are exposed. The Bighorn Mountains themselves are an erosional remnant of an asymetric, north- to northwest-trending anticlinal uplift with its steep limb forming the west edge of the Powder River Basin (Darton, 1906). The most rugged parts of the range and the maximum structural relief are found just west of Buffalo along the western edge of the study area.

Precambrian 1gneous and metamorphic rocks are exposed at the higher elevations in the core of the Bighorn uplift. Paleozolc and Mesozoic strata are exposed in the narrow, highly deformed belt along the eastern flank of the Bighorns, in the southern end of the bighorn uplift (where the mountains have less relief and elevation) and, to a lesser extent, along the east side of the Powder River Basin parallel to the west edge of the Black Hills monocline. These pre-Tertiary sediments consist of a thin sequence of carbonate and clastic marine sediments deposited along a craton-shelf boundary. Many of the Paleozoic and Mesozoic formations differ greatly in their lithologies from west to east and, to a lesser extent, from south to north within the basin. Because of these lateral variations in lithologies, different nomenclatures are often used for equivalent geologic units on opposite sides of the basin, as shown in Fig. 4 (Hodson et al, 1973). Up to $1300 \mathrm{~m}$ of Tertiary rocks lie beneath the surface of the major portion of the basin. Sedimentary rocks of the Fort Union (Paleocene) and Wasatch (Eocene) formations are extensively exposed over nearly equal areas. The Fort Union formation, exposed around the basin rim, consists of fine-grained sandstone and interbedded shale, carbonaceous shale, and coal. The Wasatch formation, exposed in the center of the basin, consists of fine- to coarse-grained, lenticular sandstones and interbedded shale, also with coal.

A regional uplift which began at the close of the Pliocene established the master stream system that exists today. Deposition ceased at that time and erosion of the sediments and volcanic debris that were deposited during late Tertiary began, reexcavating the buried mountains and removing all the Pliocene, Miocene, and Oligocene formations from the basin study area (Houston, 1969; Childers, 1970). Also, parts of older sedimentary formations which are of interest to the uranium geology of the basin (particularly the Wasatch formation) have been removed from the northern end of the Powder River Basin (Bendix Field Engineering Corp., 1976). The detailed geology of the Powder River Basin is described at some length by Darton (1906), Kohout (1957), Thomas (1957), Strickland (1958), Cooper et al (1963), Sharp et al (1964), Lowry and Cummings (1966), Houston (1969), Enyert (1970), McGrew (1971), Renfro (1971), Hodson et al (1973), Breckenridge et al (1974), Wendell et al (1976), and Bendix Field Engineering Corp. (1976) and need not be repeated here.

\section{REPORTED URANIUM OCCURRENCES IN THE STUDY AREA}

Since uranium was first discovered near Lusk (Niobrara County), Wyoming, in 1918, approximately 600 uranium mines, prospects, and occurrences have been reported throughout the state by a number of workers, including Butler et al (1962), Osterwald et al (1966), and Harshman (1968). Elevatorski (1976) has 


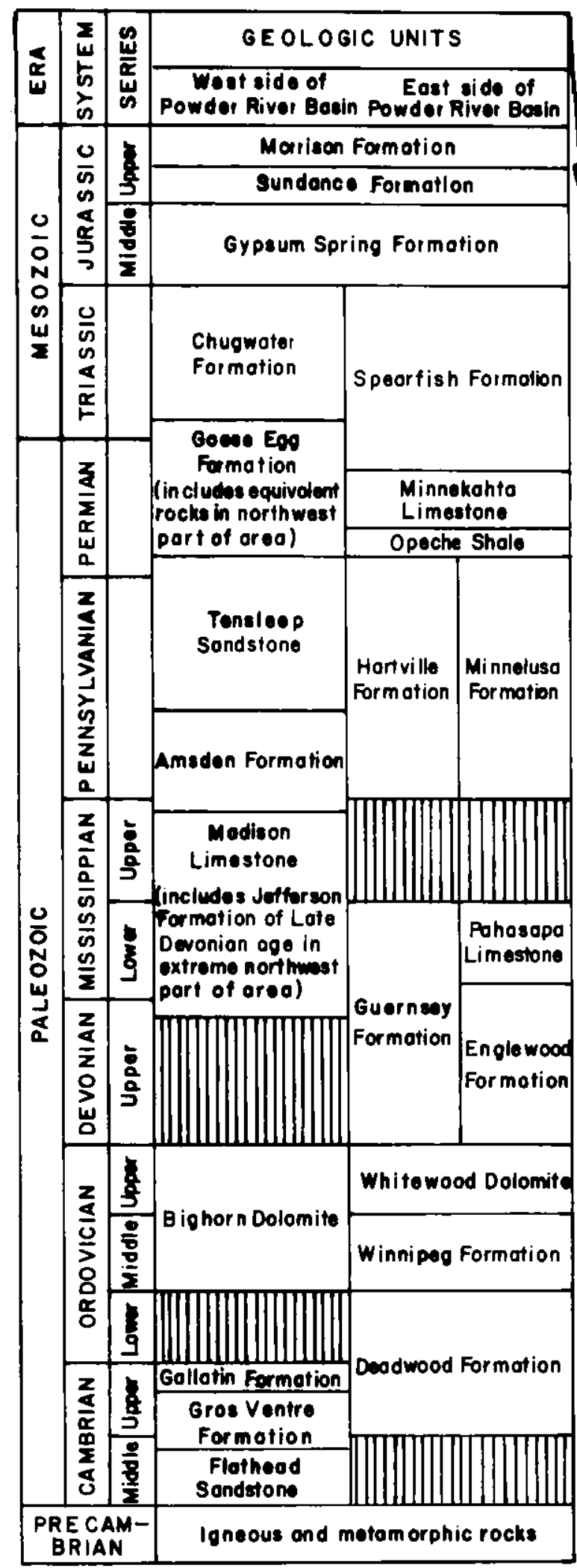

(AFTER HODSON, PEARL AND DRUSE) 1973

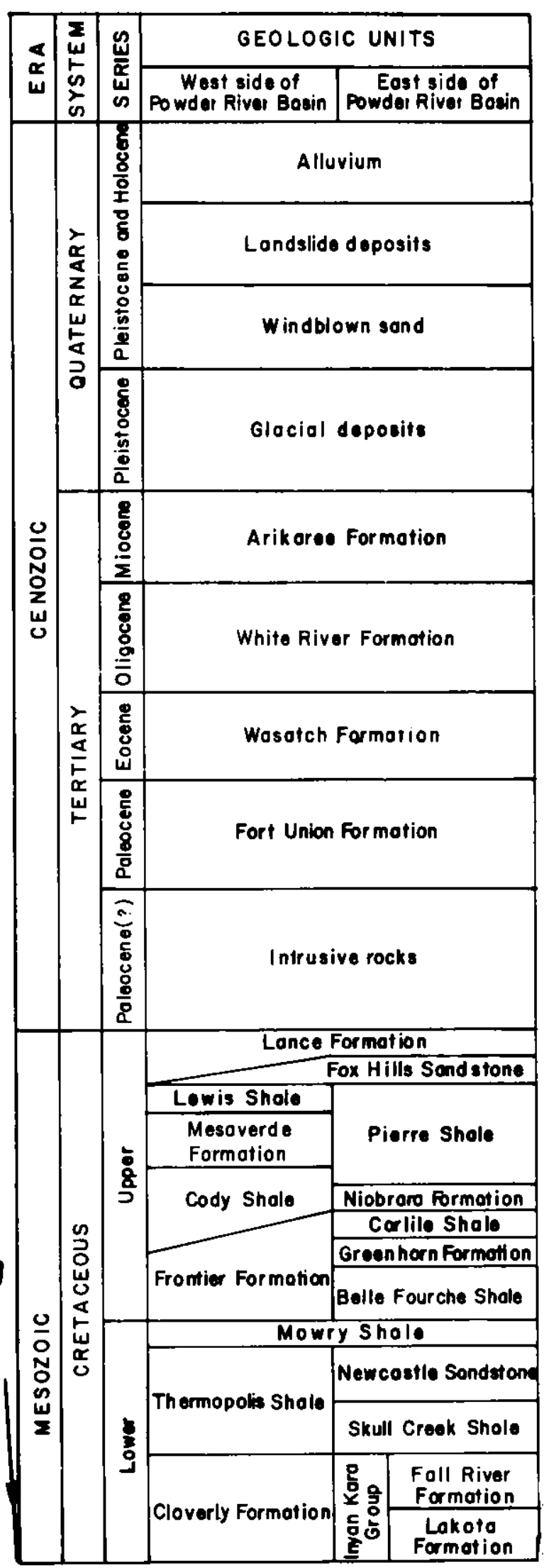

Fig. 4. Geologic units of the Powder River Basin. 
presented them in a complete and thorough review on the uranium geology and mineralization of the individual uranium districts, the areas of mineralization, and the occurrences found in 20 of the 23 counties in Wyoming. The uranium mineralization in the Mayoworth area is west of the Powder River Basin, but lies within the study area. The Northern Black Hills district, in Crook County, is outside the Powder River Basin, immediately east of the study area. The Pumpkin Buttes and Southern Powder River Basin districts, in Campbell and Converse Counties, are within the Powder River Basin but south of the study area. The Kaycee uranium district and Pine Ridge mineralized area are in the Powder River Basin and within the study area (Glass et al, 1975). The uranium districts are shown in Fig. 2; the Kaycee district and 15 other uranium occurrences are shown on Plate $I$.

Princlpal host rocks for uranium mineralization in the Powder River Basin are the permeable arkosic sandstones of the Fort Union and Wasatch formations, which were deposited by a master paleostream system that originated in the Precambrian granitic rocks of the Laramie and Granite Mountains to the south and southwest of the basin (Houston, 1969; Childers, 1970). The Fort Union host produces uraninite and coffinite; the Wasatch, carnotite, tyuyamunite, uranyl-vanadates, and uranophane (Elevatorski, 1976). In the Mayoworth area, beyond the basin, uranium mineralization occurs in the Sundance and Morrison formations (Jurassic) and the Tensleep formation (Pennsylvanian). In the northern Black Hills district, uranium has been produced from sandstones of the Lakota and Fall River formations of the Inyan Kara group (Cretaceous). Although abundant host rocks of the Fort Union and Wasatch formations are present in the northern Powder River Basin, the favorability for uranium mineralization of an area in eastern Johnson and southwest Campbell Counties (where favorable host rocks are on trend with those containing major uranium deposits in the Pumpkin Buttes and Southern Powder River Basin districts) has been rated only as "moderate," while the favorability rating for the rest of the Powder River Basin in Wyoming and Montana has been rated as "poor" (Bendix Field Engineering Corp., 1976).

The possible sources of the urarium mineralization in the Powder River Basin are discussed at length in several reports, including those by Sharp and Gibbons (1964), Houston (1969), Davis (1970), Harshman (1970), DeNault (1974), Langen and Kidwell (1974), and Bendix Field Engineering Corp. (1976). Most papers favor as the source the volcanic debris of the Oligocene White River formation (which once covered the basin), the arkosic host sandstones themselves, the Precambrian granitic rocks of the Granite and Laramie Mountains (from which the arkoses were derived), or some combination of these. However, there are no connections suggested between the uranium mineralization in the Powder River Basin and that found in the Black Hills.

The reported uranium occurrences in the Powder River Basin study area, as shown on Plate $I$, are set forth in Table $I$. Comments concerning the principal mines, host rocks, and type of occurrence or major uranium minerals at each location are also included in this table.

\section{STATISTICAL DATA AND EVALUATION OF URANIUM CONCENTRATIONS IN WATER}

A total of 1178 water samples were collected and analyzed in this study. The sample locations are shown on Plate II. Note on Plate II that 200 000 has been subtracted from each of the sample location numbers as set forth in the data listings of Appendix A. The uranium concentrations are shown graphically for the waters on Plate IrI. These two plates overlay the 
TABLE I

REPORTED URANIUM OCCURRENCES IN THE POWDER RIVER BASIN STUDY AREA

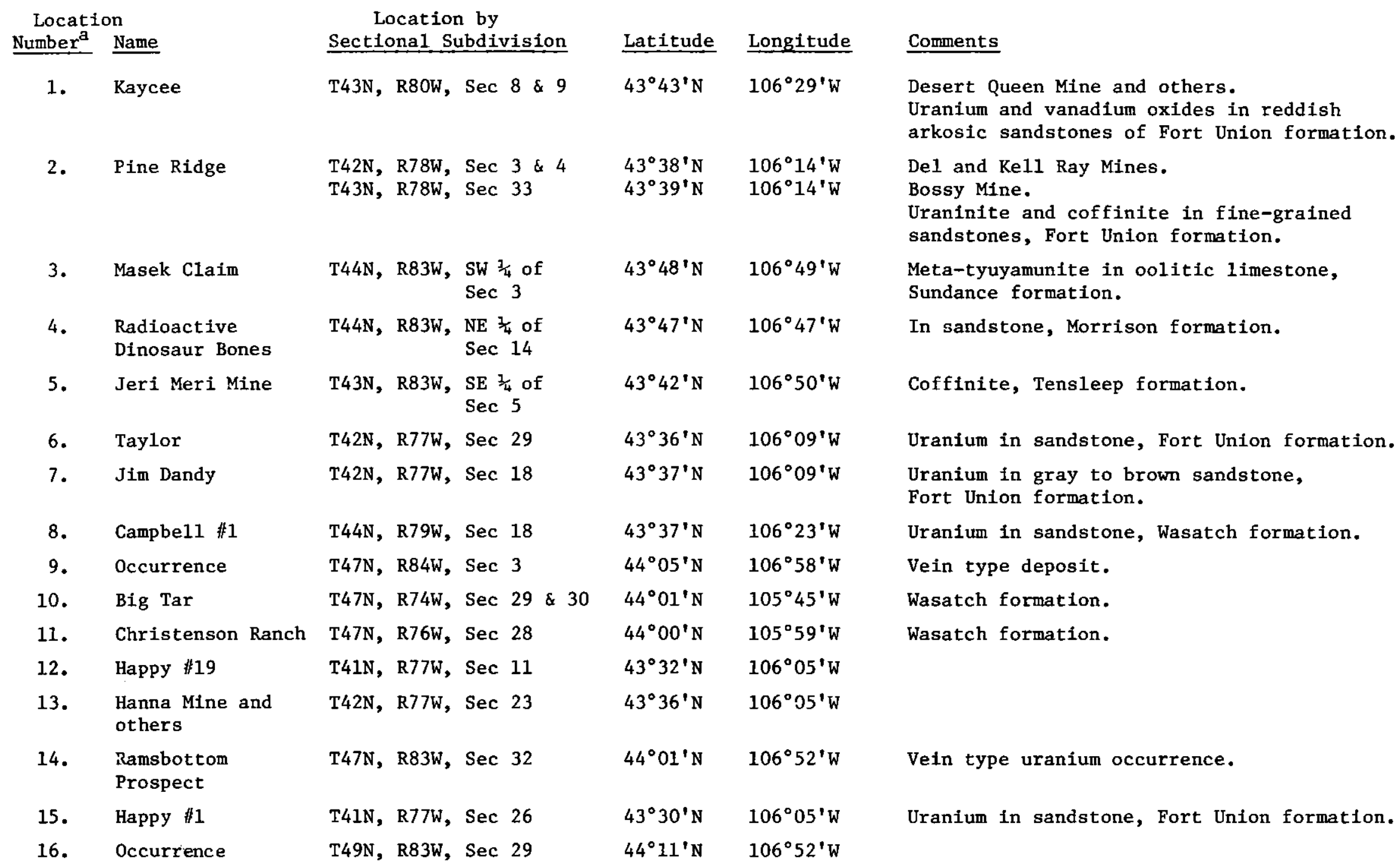

ased to indicate locations on Plate $I$. 
geologic map, Plate $I$, and the portions of the Gillette, Sheridan, and Arminto NTMS topographic maps included on them. The location, site description, field measurements, and uranium content of each water sample are listed in Appendix A. All waters analyzed by fluorometry were done in duplicate with the uranium values shown being the average of the two determinations. The method utilized has been described in detail by Hues et al (1977). Occasionally, one split of a sample would result in a concentration at or slightly above the lower detectable limit ( 0.2 parts per billion ( $\mathrm{ppb})$ ), while the other was below the limit. In such cases, the lower concentration of uranium ( $<0.2 \mathrm{ppb})$ was arbitrarily assigned a value of $0.1 \mathrm{ppb}$, then averaged with the higher value. All of the uranium values for waters in the data listings of Appendix A between 0.1 and $0.2 \mathrm{ppb}$, then, were arrj.ved at in this manner. Samples with uranium concentrations above $10 \mathrm{ppb}$, which could not readily be analyzed by fluorometry without recalibration, were done by delayed-neutron counting (DNC) in the manner described by Sharp and hamodt (1976). Statistical analyses of samples treated in the same way from New Mexico (Whiteman, 1977) and from Colorado (Sharp and Aamodt, 1976) have shown that there is no significant difference between the uranium concentrations in water determined at the LASL by fluorometry and those determined by DNC. The field data for a few samples are missing; in such cases, blanks occur in the data listings of Appendix A. An explanation of the codes used in the data listings of Appendices $A$ and $B$ is given in Appendix C. All samples were treated in accordance with the standard LASL procedures set forth in Appendix $D$; the waters were filtered through a $0.45-\mu$ filter paper and acidified (to $\mathrm{pH} \leq 1$ ) with reagent-grade nitric acid.

The number of samples in the population, the minimum, maximum, and mean uranium concentration values, and the standard deviation for each water sample type reported are listed in Table II. Four samples--stream samples 200148 $(212.2 \mathrm{ppb})$ and $200676(276.4 \mathrm{ppb})$, natural pond sample 200072 (204.3 ppb), and artificial pond sample 200934 (295.6 ppb)--are not included in Table II or in the frequency histogram for water (Fig. 5a) because their uranium concentrations are nearly two or more times the next highest values and markedly distort the mean and standard deviation for these sample types. When evaluating uranium results from mixed water sample populations (different source types), it is important to recognize that they are usually not directly comparable. Several reasons for this are alluded to by Aamodt (1977) in his discussion of the geochemical nature of uranium. However, the results of the

TABLE II

\section{URANIUM CONCENTRATIONS BY TYPE OF WATER SAMPLE}

\begin{tabular}{|c|c|c|c|c|c|}
\hline $\begin{array}{l}\text { Sample } \\
\text { Type }\end{array}$ & $\begin{array}{r}\text { Number of } \\
\text { Sammples }\end{array}$ & $\begin{array}{l}\text { Minimum } \\
\text { (ppb) }\end{array}$ & $\begin{array}{l}\text { Maximum } \\
(\mathrm{ppb})\end{array}$ & $\begin{array}{l}\text { Mean } \\
(\mathrm{ppb}) \\
\end{array}$ & $\begin{array}{l}\text { Standard } \\
\text { Deviation }\end{array}$ \\
\hline Ail waters * & 1174 & 0.1 & 115.3 & 6.51 & 14.8 \\
\hline Springs & 142 & 0.1 & 59 & 4.98 & 10.6 \\
\hline Wells & 720 & 0.1 & 115.3 & 5.5 & 14.2 \\
\hline Streams & 109 & 0.1 & 75.1 & 8.09 & 15.8 \\
\hline Natural Ponds* & 18 & 0.1 & 7.68 & 4.33 & 6.42 \\
\hline Artificial Ponds & 185 & 0.1 & 106.5 & 10.76 & 18.68 \\
\hline
\end{tabular}

* Water samples from two streams, one natural pond, and one artificial pond, all having $>200 \mathrm{ppb}$ uranium, are not included. 
analyses of water samples from the Powder River Basin indicate that they are somewhat more statistically comparable than would normally be expected.

Therefore, for this report, all water samples will be treated together.

The distribution of uranium concentrations in the water is shown in the histogram of Fig. 5a. Note that all water samples having a uranium concentration above $45 \mathrm{ppb}$ are grouped together at the extreme right of the histogram. other than the four water samples with the highest values that are excluded, the highest concentration of uranium found in water is $115.3 \mathrm{ppb}$ and occurs in a sample from a well (200366). The distribution of uranium concentrations by ranges for all waters is shown in Table III.

There are water samples from 41 locations within the study area having a uranium concentration greater than three standard deviations above the mean (i.e., $>50 \mathrm{ppb}--$ represented by the two largest symbols on the water overlay). These are from three springs, six streams, twenty-one wells, one natural pond, and ten artificial ponds. At three standard deviations above the mean, the samples from all of these locations can be considered anomalous (Aamodt, 1977). Thirty-five of the 41 locations providing anomalous water samples are spread across the major portion of the basin of the study area in the Wasatch and Fort Union formations, forming six clusters, most of which contain several additional locations with water samples having above-average uranium. These two formations, particularly the Wasatch, are the uranium producers in the Pumpkin Buttes and Southern Powder River districts.

One cluster of six anomalous well water sites is at the southern edge of the study area, between the Powder River and Crazy Woman Creek in the Four Mile Creek drainage. This area is in the "on trend" region described by the Bendix Field Engineering Corp. (1976) as having the greatest potential for uranium in the Powder River Basin north of the existing districts. A second cluster is around and southwest of Gillette, in the Hoe Creek-Horse CreekCaballo Creek and Wild Horse Creek drainages, where there are seven anomalous water samples: one well, one stream, three springs, and one natural and one artificial pond. A third cluster is east of Gillette, in the Donkey Creek drainage, where there are three anomalous well water samples. A fourth cluster is in the general area of the Buffalo Creek-Clear Creek-Crazy Woman Creek drainages, west of the Powder River, and the SA Creek drainage, east of the Powder River, from northeast of Buffalo on northward to Montana, where there are 11 anomalous sites: six artificial ponds, two wells, and three

TABLE III

URANIUM CONCENTRATIONS BY RANGES FOR WATERS

(Mean: $7.3 \mathrm{ppb}$; standard Deviation: $20.5 \mathrm{ppb}$ )

\begin{tabular}{rr|rr} 
Range (ppb) & No. & Range (ppb) & No. \\
\hline $0.0-10.5$ & 1008 & $63.2-73.7$ & 8 \\
$10.5-21.1$ & 63 & $73.7-84.2$ & 2 \\
$21.1-31.6$ & 32 & $84.2-94.7$ & 5 \\
$31.6-42.1$ & 25 & $94.7-105.3$ & 1 \\
$42.1-52.6$ & 15 & $105.3-115.8$ & 5 \\
$52.6-63.2$ & 10 & $115.8-200.0$ & 0 \\
& & $200.0-295.8$ & 4
\end{tabular}




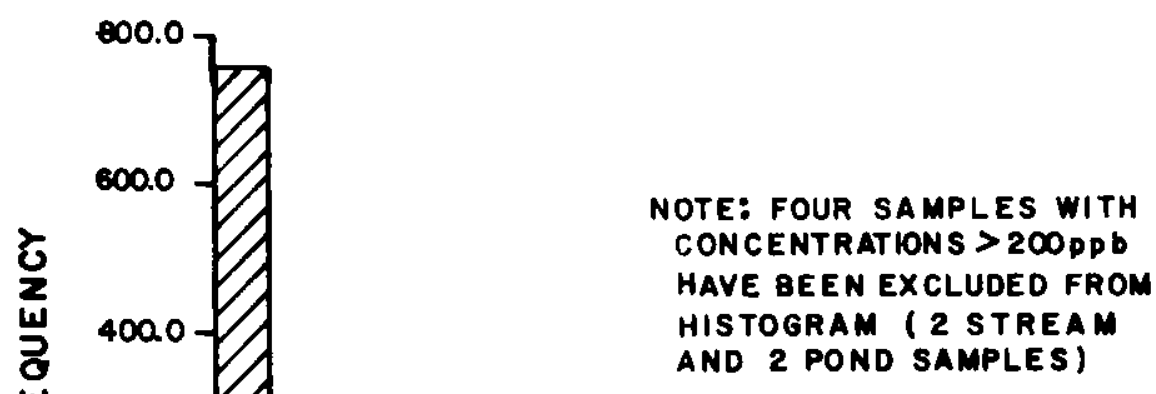

ALL SAMPLES $>$ 45.0, EXCEPT AS NOTEO

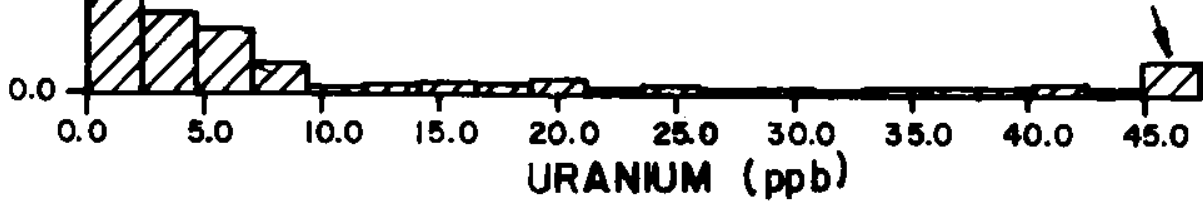

MAXIMUM $=115.30 \quad$ MEAN $=6.51$

MINIMUM $=0.10$

SAMPLE SIZE $=1174 \quad$ STANDARD DEVIATION $=14.80$

a. FREQUENCY HISTOGRAM FOR WATERS

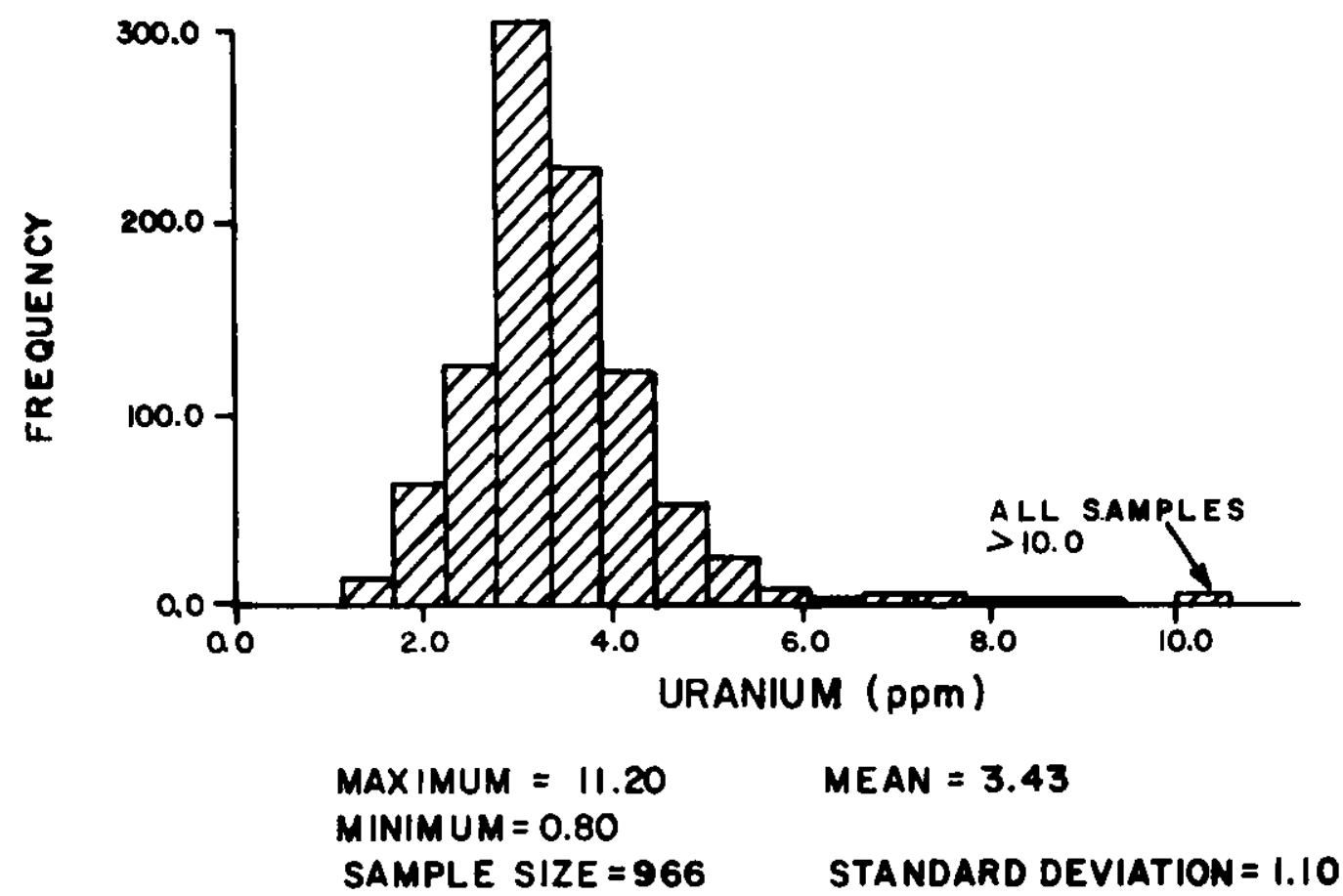

b. FREQUENCY HISTOGRAM FOR SEDIMENTS

Fig. 5

Distribution of uranium concentrations in water and sediment samples. 
streams. The fifth cluster, containing two anomalous well sites, is in the Hanging Woman Creek drainage along the Montana state line. And lastly, the general area around Sherldan (Badger, Prairle Dog, Wlldcat, Little Goose, and Big Goose Creek drainages) has six anomalous sites: two artificial ponds, three wells, and one stream.

There is one anomalous cluster that is not in the Wasatch and Fort Union formations. In this area, along US 87 from $16 \mathrm{~km}$ north of Kaycee on northward to Crazy Woman Creek, there are six anomalous sites: sample locations 201090, 201093, and 201097 (all wells) are in the Lewis, Fox Hills, and Lance formations, undivided (Cretaceous); sample location 200986 (an artificial pond water) is in the Fort Union formation; sample location 200974 (a well) is in the Mesaverde formation (Cretaceous); and sample location 201863 (a stream) is in the Cloverly and Morrison formatioris.

Three of the very high water saniples (>200 ppb uranium) are in the Wasatch formation: sample location 200072 is a natural pond, $3 \mathrm{~km}$ west of Gillette with $204.3 \mathrm{ppb}$ uranium; sample location 200676 is a flowing stream in the Coal Gulch drainage with $276.4 \mathrm{ppb}$ uranium; and sample location 200934 is an artificial pond in the Badger Creek drainage with $295.6 \mathrm{ppb}$ uranium, the highest uranium concentration found in the study. The fourth very high water sample, from location 200148 , is from a flowing stream in the Bitter Creek drainage in the Fort Union formation and has $212.2 \mathrm{ppb}$ uranium. These four samples are spread over the study area; two are in the north part and two are in the southcentral part.

Only six of the 16 uranium occurrences shown on Plate I (Nos. 3, 4, 5, 7,8 , and 10) were detected in the water sampling results. Three others (Nos. 11,14 , and 16) should probably have been detected by the samples as taken but were not; seven (Nos. 1, 2, 6, 9, 12, 13, and 15) could not have shown up either because the occurrence was at the edge of the map (and the drainage was off the map) or a sample was not taken where originally specified on the drainage due to access limitations. Table IV gives the uranium value and location of the sample (if any) taken nearest the known occurrences.

Because most of the sampling was done over a four-month period (65 samples were collected two months later, for a total of six months), a study of the dependency of the uranium concentrations on the time of sampling was done on the ground waters (represented by wells) and surface waters (represented by streams) to see if there was any variance introduced by time. Beckman (1977) found that the uranium concentrations in well waters had no time dependency; this finding is supported by that reported by Fix (1956) for ground waters. He also found that the uranium concentrations in stream waters may be dependent on time. But, since the uranium in the streams was also found to be dependent on elevation and slope (samples from the higher elevations in the west side of the study area had lower values), the effect of the time of sampling on the uranium values could not be determined for certain. A further check of the fleld data reveals that all 111 stream water samples were taken between March 28, 1976, and July 14, 1976. Of these, 84 were taken by June 2 and the remaining 27 from July 4 to July 14 . All 24 of the high uranium values in stream waters ( $>10 \mathrm{ppb}$ ) were spread evenly over the March 28 to June 2 period; none were collected during the July period, which had less rainfall. Therefore, it is concluded that the variation of uranium values in surface waters is due mainly to elevation and ground slope, rather than time (or the change in season experienced). 
TABLE IV

URANIUM OCCURRENCES REFLECTED IN WATER RESULTS

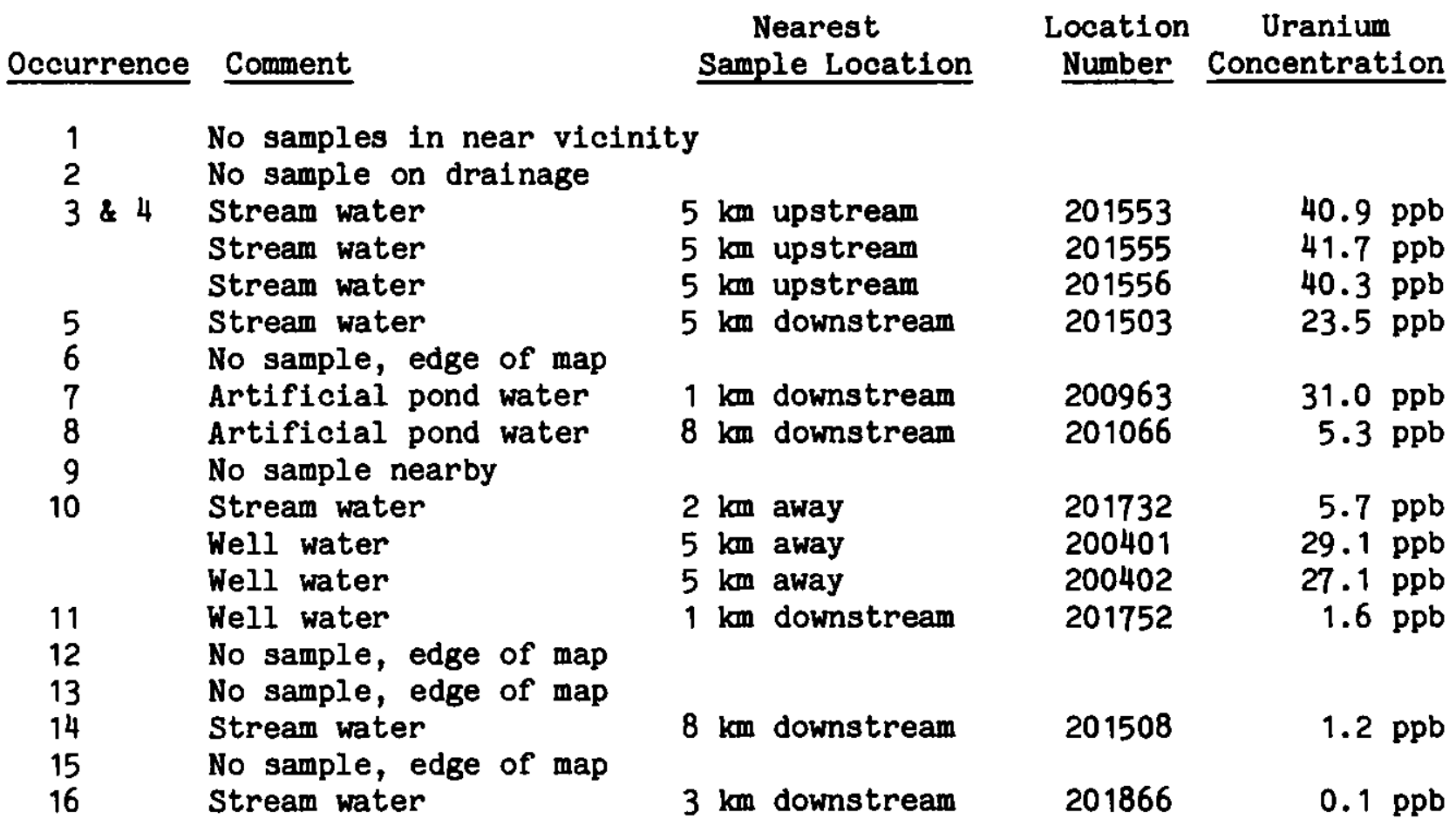

of the 186 artificial pond waters that were sampled, 50 contained greater than $10 \mathrm{ppb}$ uranium and 10 were anomalous ( $>50 \mathrm{ppb}$ ). These data appear to be ercosiaging at first glance, particularly since all of them are in the uraniferous Wasatch or Fort Union formations. However, the uranium concentration in artificial ponds can be misleading as the effect of evaporation can increase it by a factor of 100 or more (Fix, 1956). Therefore, these sample locations should be evaluated carefully.

\section{STATISTICAL DATA AND EVALUATION OF URANIUM CONCENTRATIONS IN SEDIMENT}

A total of 966 sediment samples were collected and analyzed in this study. The sample locations are shown on Plate II. Again, note on Plate II that 200000 has been subtracted fror the sample location numbers as set forth in Appendix B. The uranium concentrations are shown graphically for sediments on Plate IV. Sample locations with uranium concentrations equal to the values that divide the various symbol sizes on Plate IV will appear as the larger symbol. These two plates overlay the geologic map, Plate I, and the portions of the Gillette, Sheridan, and Arminto NTMS topographic maps as indicated on the overlays. The location, site description, field measurements, and uranium content in parts per million ( $\mathrm{ppm}$ ) for each sediment sample are listed in Appendix B. The field data for a few samples are missing; in such cases, blanks cccur in the data listings. All of the sediment samples were analyzed by DNC in the manner described by Sharp and Aamodt (1976). The 12 samples listed in Appendix B as being "Other" sample types (numerical identification 99) are all dry spring sediments. An explanation of the codes used in the 
data listings is given in Appendix C. All sediment samples were treated in accordance with the standard LASL procedure (Appendix D); they were dried and then sieved to -100 mesh using a standard USA No. 100 stainless steel screen.

The number of samples in the population, the minimum, maximum, and mean uranium concentration values, and the standard deviation of each sample type reported are listed in Table $\mathrm{V}$. The distribution of uranium concentrations in the sediments is shown in the frequency histogram of Fig. 5b. Note that all sediment samples having a uranium concentration greater than $10 \mathrm{ppm}$ are grouped together at the extreme right of the histogram. The highest concentration of uranium in sediment is $11.2 \mathrm{ppm}$ in a dry stream sediment located on the north fork of Cabin Creek, in the Fort Union formation (200723). The uranium concentrations by range for sediments are given in Table VI. There are 19 sediment samples with a uranium concentration greater than three standard deviations above the mean (i.e., $>6.7 \mathrm{ppm}$ uranium, represented by the three largest symbols on the sediment overlay). They come from a wet spring, two wet and nine dry streams, two wet natural ponds, and five wet artificial ponds. At three standard deviations above the mean, the samples from all of these locations can be considered anomalous (Aamodt, 1977).

There is only one cluster of anomalous sediment samples. It is in the area between the North Fork, Middle Fork, and Red Fork of the Powder River south of Mayoworth Junction and contains four sites: sample location 201189 (wet natural pond) is in the Frontier formation (Cretaceous); sample locations 201471 (wet artificial pond) and 201472 (wet spring) are in the Cloverly/ Morrison formations (Cretaceous/Jurassic); and sample location 201473 (wet artificial pond) is in the Mowery and Thermopolis shales, undivided (Cretaceous). All the remaining anomalous sediment samples are spread out over most of the study area, and they do not cluster or indicate areas of interest except as individual, isolated points. However, individual, isolated anomalous points should be evaluated very carefully and cannot be assigned the weight of interest that is justified by a cluster of anomalously high values which give form and extent to an anomalous area and thus allow some evaluation in light of the geology, structure, vegetation, and other areal parameters (Grimbert and Loriod, 1968).

Only 2 of the 16 uranium occurrences shown on Plate I (Nos. 2 and 7) were detected in the sediment sampling results. A dry stream sediment from

\section{TABLE V}

\section{URANIUM CONCENTRATIONS BY TYPE OF SEDIMENT SAMPLE}

\begin{tabular}{|c|c|c|c|c|c|}
\hline $\begin{array}{l}\text { Sample } \\
\text { Type }\end{array}$ & $\begin{array}{c}\text { Number } \\
\text { of Samples }\end{array}$ & $\begin{array}{l}\text { Minimum } \\
\text { (ppm) }\end{array}$ & $\begin{array}{l}\text { Maximum } \\
(\mathrm{ppm}) \\
\end{array}$ & $\begin{array}{r}\text { Mean } \\
\text { (ppm) } \\
\end{array}$ & $\begin{array}{l}\text { Standard } \\
\text { Deviation }\end{array}$ \\
\hline $\begin{array}{l}\text { All sediments } \\
\text { Wet Spring } \\
\text { Wet Stream } \\
\text { Wet Natural Pond } \\
\text { Wet Artificial Pond } \\
\text { Dry Stream } \\
\text { Dry Natural Pond } \\
\text { Dry Artificial Pond } \\
\text { Other (Dry Spring) }\end{array}$ & $\begin{array}{r}966 \\
131 \\
108 \\
20 \\
170 \\
415 \\
9 \\
101 \\
12\end{array}$ & $\begin{array}{l}0.8 \\
0.8 \\
1.3 \\
2.2 \\
1.6 \\
1.4 \\
2.5 \\
1.2 \\
1.8\end{array}$ & $\begin{array}{r}11.2 \\
6.9 \\
10.3 \\
8.1 \\
8.7 \\
11.2 \\
4 . \\
5.6 \\
3.5\end{array}$ & $\begin{array}{l}3.43 \\
2.98 \\
3.15 \\
4.0 \\
3.5 \\
3.6 \\
3.6 \\
3.5 \\
2.73\end{array}$ & $\begin{array}{l}1.09 \\
0.98 \\
1.3 \\
1.4 \\
0.97 \\
1.14 \\
0.58 \\
0.73 \\
0.59\end{array}$ \\
\hline
\end{tabular}


URANIUM CONCENTRATIONS BY RANGES FOR SEDIMENTS

\author{
966 Sediments \\ (Mean: $3.4 \mathrm{ppm}$; Standard Deviation: $1.1 \mathrm{ppm}$ )
}

$\begin{array}{cc}\text { Range (ppm) } & \begin{array}{c}\text { Number } \\ \text { of Samples }\end{array} \\ 0.5-1.1 & \\ 1.1-1.7 & 14 \\ 1.7-2.2 & 64 \\ 2.2-2.7 & 125 \\ 2.7-3.3 & 303 \\ 3.3-3.9 & 229 \\ 3.9-4.4 & 122 \\ 4.4-5.0 & 53 \\ 5.0-5.6 & 24\end{array}$

$\begin{array}{rr}\text { Range (ppm) } & \begin{array}{r}\text { Number } \\ \text { of Samp }\end{array} \\ 5.6-6.1 & \\ 6.1-6.7 & \\ 6.7-7.2 & 3 \\ 7.2-7.8 & 5 \\ 7.8-8.3 & 5 \\ 8.3-8.9 & 1 \\ 8.8-9.4 & 2 \\ 9.4-10.0 & 1 \\ 10.0-11.2 & 0 \\ & 5\end{array}$

sample location 201068 with $7.2 \mathrm{ppm}$ uranium is located $6 \mathrm{~km}$ downstream from occurrence No. 2, and a wet artificial pond sediment from sample location 200963 with $5.5 \mathrm{ppm}$ uranium is located within $1 \mathrm{~km}$ of occurrence No. 7 . It might have been expected that five other occurrences (Nos. 1, 6, 10, 14, and 16) would have been detected by the samples taken, but they were not. The nine remaining occurrences (Nos. $3,4,5,8,9,11,12,13$, and 15) could not have been detected, either because the drainages around them were off the map or because no sample was taken as originally specified on the drainage due to access limitations or restrictions on the type of sample specified or available.

\title{
VI. CONCLUSIONS
}

The uranium concentrations and chemical properties of the ground waters from this study are, in general, similar to what Sharp and Gibbons (1964) found in their ground water samples taken near the Monument Hill deposits in the Southern Powder River Basin uranium district. They found the ground waters from that part of the Powder River Basin to be high in dissolved solids and slightly basic and to contain more than the common amount of uranium. Nine of their 32 samples were reported as anomalous and ranged from 41 to 220 ppb; samples from the Wasatch formation averaged $28 \mathrm{ppb}$ and from the Fort Union, $8 \mathrm{ppb}$. These data are slightly lower than that reported by Denson et al (1956) in ground water samples taken from 56 wells and one spring in the Pumpkin Buttes/Southern Powder River Basin uranium districts. They concluded, from their samples from these mineralized areas, which averaged $84 \mathrm{ppb}$ uranium, that ground waters from mineralized areas may contain as much as 10 to $250 \mathrm{ppb}$ uranium. Fix (1956) states that a rough guide to waters requiring further investigation for the Colorado Plateau is $4 \mathrm{ppb}$ in surface waters and $5 \mathrm{ppb}$ in ground waters, and that uraniferous areas generally contain 1 to 10 $\mathrm{ppb}$ uranium in surface waters and 1 to $120 \mathrm{ppb}$ uranium in ground waters. Harshman (1968) reported that an area that contains $10 \mathrm{ppb}$ uranium in the 
ground water may contain uranium deposits and that areas with less than $5 \mathrm{ppb}$ are most likely barren. Based on these criteria and the uranium data of this report, which are similar to those reported from the southern part of the Powder River Basin where uranium has been found, it is concluded that the anomalous water and sediment sample clusters previously mentioned are areas in the northern Powder River Basin that merit further study and examination even though there are no known uranium occurrences nearby.

\section{ACKNOWLEDGMENTS}

The writer gratefully acknowledges the following LASL employees whose assistance was essential to the preparation of this report: Arthur L. Henicksman, Donna Hedrick, Loyola Gurule, Walter Sandoval, and Patricia Bacon of the Analytical Chemistry Group, CMB-1, who performed the fluorometric analyses of the water samples; Merle E. Bunker, Michael M. Minor, Walter $\mathrm{K}$. Hensley, and James S. Bergauer of the Research Reactor Experiments Group, P-2, who performed the delayed-neutron counting of the high water ( $>10 \mathrm{ppb}$ uranium) and sedinert $\cdots$ inles; and Carlotta McInteer, Jesse Cheadle III, and Richard J. Beckman of the Energy Systems and Statistics Group, Q-12, who were responsible for data storage and control, statistical treatments, and graphic plots. A special thanks goes to the following members of the Geochemical Applications Group, G-5: Patricia O'Rourke for typing and editing the text, Alcario Marquez for preparing the figures and plates included in the report, and Robert $R$. Sharp, Jr. for reviewing the entire report and making suggestions for its improvement. Finally, sincere appreciation goes to the landowners and the US Forest Service who granted permission for access to their lands for the purpose of sampling. Without their kind cooperation this reconnaissance could not have been done. 


\section{REFERENCES CITED}

Aamodt, P. L., 1977, Uranium hydrogeochemical and stream sediment reconnaissance in Lincoln and Flathead Counties, northwest Montana, GJBX-48(77), US ERDA, Grand Junction, CO, 138 p.

Becker, C. F. and Alyea, J. D., 1964a, Temperature probabilities in Wyoming, Univ. of Wyoming Agr. Exp. Station Bull. 415, Laramie, WY, 157 p.

Becker, C. F. and Alyea, J. D., 1964b, Precipitation probabilities in Wyoming, Univ. of Wyoming Agr. Exp. Station Bull. 416, Laramie, WY, 97 p.

Beckman, R. J., 1977, Group Q-12, Los Alamos Scientific Laboratory, Los Alamos, NM, personal communication.

Bendix Field Engineering Corporation, 1976, Uranium favorability of the Fort Union and Wasatch formations in the northern Powder River Basin, Wyoming and Montana, GJBX-58(76), Grand Junction, CO, 97 p.

Breckenridge, R. M., Glass, G. B., Root, F. K., and Wendell, W. G., 1974, Geologic map atlas and summary of land, water, and mineral resources, Campbell County, Wyoming, Geol. Survey of Wyoming County Resource Series CRS-3, Laramie, WY, 9 p.

Butler, A. P., Jr., Finch, W. I., and Twenhofel, W. S., 1962, Epigenetic uranium in the United States, USGS Mineral Investigation Resource Map MR-21 ( $1: 3168000$ scale), Washington, DC.

Childers, M. 0., 1970, Uranium geology of the Kaycee area, Johnson County, Wyoming, Wyoming Geol. Assoc. Guidebook, 22nd Annual Field Conf., Symposium on Wyoming sandstones, Casper, WY, pp. 13-20.

Cooper, G. G., Cardinal, D. F., Lorenz, H. W., and Lynn, J. R., Eds., 1963, Northern Powder River Basin, Wyoming Geol. Assoc. and Billings Geol. Assoc., ist Joint Field Conf. Guidebook, Casper, WY, 204 p.

Darton, N. H., 1906, Geology of the Bighorn Mountains, USGS Prof. Paper 51, Washington, DC, 129 p.

Davis, J. F., 1970, Uranium deposits of the Powder River Basin, Wyoming, Symposium on Wyoming sandstones, Wyoming Geol. Assoc. Guidebook, 22nd Annual Field Conf., Casper, WY, pp. 21-29.

DeNault, K. J., 1974, Origin of sandstone type uranium deposits in Wyoming, Ph.D. Dissertation, Stanford University, Palo Alto, CA, 352 p.

Denson, N. M., Zeller, H. D., and Stephens, J. G., 1956, Water sampling as a guide in the research for uranium deposits and its use in evaluating widespread volcanic units as potential source beds for uranium, USGS Prof. Paper 300, Washington, DC, pp. 673-680.

Elevatorski, E. A., Comp., 1976, Uranium Guldebook for Wyoming, Minobras, Dana Point, CA., 63 p. 
Enyert, R. L., Ed., 1970, Symposium on Wyoming sandstones, Wyoulng Geol. Assoc. Guldebook, 22nd Annual Field Conf., Casper, WY, 292 p.

Fix, P. F., 1956, Hydrogeochemical exploration for uranium, USGS Prof. Paper 300, Washington, DC, pp. 667-671.

Glass, G. B., Wendell, W. G., Root, F. K., and Breckenridge, R. M., 1975, Energy Resources Map of Wyoming (1:500 000 scale), Geol. Survey of Wyoming, Laramie, WY.

Grimbert, A. and Loriod, R., 1968, Geochemical prospecting for uranium, AEC-tr-7579 (trans. from French) US AEC, Tech. Info. Center, Springfield, VA, $38 \mathrm{p}$.

Harshman, E. N., 1968, Uranium deposits of Wyoming and South Dakota, in: Ore deposits of the United States, 1933-1967, Am. Inst. Min. Met. Engr., v. 1, New York, pp. 817-831.

Harshmnan, E. N., 1970, Uranium ore rolls in the United States, in: Uranium Exploration Geology, Proc. of a Panel, IAEA, Vienna, pp. 219-232.

Hodson, W. G., 1971, Chemical analyses of ground water in the Powder River Basin and adjacent areas, northeastern Wyoming, USGS Basic Data Report, Wyoming Dept. Bcon. Planning and Development, Cheyenne, WY, 20 p.

Hodson, W. G., Pearl, R. H., and Druse, S. A., 1973, Water resources of the Powder River Basin and adjacent areas, northeast Wyoming, USGS Hydrologic Invest. Atlas HA-465, Washington, DC.

Houston, R. S., 1969, Aspects of the geologic history of Wyoming related to the formation of uranium deposits, Contributions to Geology, Wyoming Uranium Issue, Untv. of Wyoming, Laramie, WY, v. 8, no. 2, pt. 1, pp. 67-80.

Hues, A. D., Hentcksman, A. L., Ashley, W. H., and Romero, D., 1977, The fluorometric determination of uranium in natural waters, GJBX-24(77), US ERDA, Grand Junction, CO, $11 \mathrm{p}$.

Kohout, F. A., 1957, Geology and ground water resources of the Kaycee irrigation project, Johnson County, Wyoming, USGS Water Supply Paper 1360-E, Washington, DC, 374 p.

Langen, R. E. and Kidwell, A. L., 1974, Geology and geochemistry of the Highland uranium deposit, Converse County, Wyoming, Mountain Geologist, v. 11, no. 2 , pp. 85-93.

Lowry, M. E. and Cumings, T. R., 1966, Ground water resources of Sheridan County, Wyoming, USGS Water Supply Paper 1807, Washington, DC, 77 p.

McGrew, P. 0., 1971, The Tertiary history of Wyoming, Wyoming Tectonics Symposium, Wyoming Geol. Assoc. Guidebook, 23rd Annual Field Conf., Casper, WY, pp. 29-34. 
National Oceanic and Atmospheric Administration, 1976, Climatological Data, Wyoming, US Dept. of Commerce, v. 85, nos. 4-10, Ashville, NC.

Osterwald, F. W., Osterwald, D. B., Long, J. S., Jr., and Wilson, W. H., 1966, Mineral resources of Wyoming, Geol. Survey of Wyoming Bull. 50, Laramie, WY, $281 \mathrm{p}$.

Renfro, A. R., Ed., 1971, Symposium on Wyoming Tectonics, Wyoming Geol. Assoc. Guidebook, 23rd Annual Field Conf., Casper, WY, 187 p.

Renfro, H. B. and Feray, D. E., 1975, Geological highway map of the northern Rocky Mountain region (1:158 400 scale), Am. Assn. Petr. Geol. Map No. 5.

Sharp, R. R., Jr., 1977, The LASL approach to uranium geochemical reconnalssance, paper presented at the March 1977 Symposium on Hydrogeochemical and Stream Sediment Reconnaissance for Uranium in the United States, sponsored by the ERDA GJO and Bendix Field Eng. Corp., Grand Junction, C0, 21 p.

Sharp, R. R., Jr. and Aamodt, P. L., 1976, Uranium concentrations in natural waters, South Park, Colorado, GJ-BX-35(76), US ERDA, Grand Junction, CO, $49 \mathrm{p}$.

Sharp, W. N. and Gibbons, A. B., 1964, Geology and uranium deposits of the southern part of the Powder River Basin, Wyoming, USGS Bulletin 1147-D, Washington, DC, $60 \mathrm{p}$.

Sharp, W. N., McKay, E. J., McKeown, F. A., and White, A., M., 1964, Geology and uranium deposits of the Pumpkin Buttes area of the Powder River Basin, USGS Bulletin 1107-H, Washington, DC, pp. 541-638.

Strickland, J., Ed., 1958, Powder River Basin, Wyoming Geol. Assoc. Guidebook, 13th Annual Field Conf., Casper, WY, 341 p.

Thomas, H. D., 1957, Geologic history and structure of Wyoming, Contribution of the Geol. Survey of Wyoming Reprint No. 18 (originally published in 1949), Laramie, WY, 11p.

Wendell, W. G., Glass, G. B., Breckenridge, R. M., Root, F. X., Lageson, D., and the Remote Sensing Lab., Univ, of Wyoming, 1976, Geologic map atlas and summary of land, water, and mineral resources, Johnson County, Wyoming, Geol. Survey of Wyoming County Resource Series CRS-4, Laramie, WY.

Whitcomb, H. A., Cummings, T. R., and McCullough, R. A., 1966, Ground water resources and geology of northern and central Johnson County, Wyoming, USGS Water Supply Paper 1806, Washington, DC, 99 p.

Whiteman, D. E., 1977, Group Q-12, Los Alamos Sctentific Laboratory, Los Alamos, MM, personal communication. 


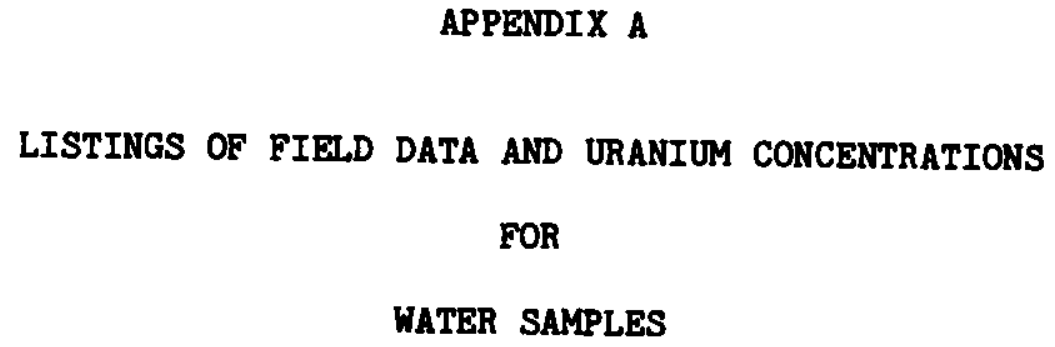

ITEM A-I

WATER SAMPLES ANALYZED BY FLUOROMETRY

(Pages 24 through 43)

ITEM A-II

WATER SAMPLES ANALYZED BY DELAYED-NEUTRON COUNTING

(Pages 44 through 47) 
LASL Uranium Hydrogeochemical and Stream Sediment Reconnaissance Data Listing

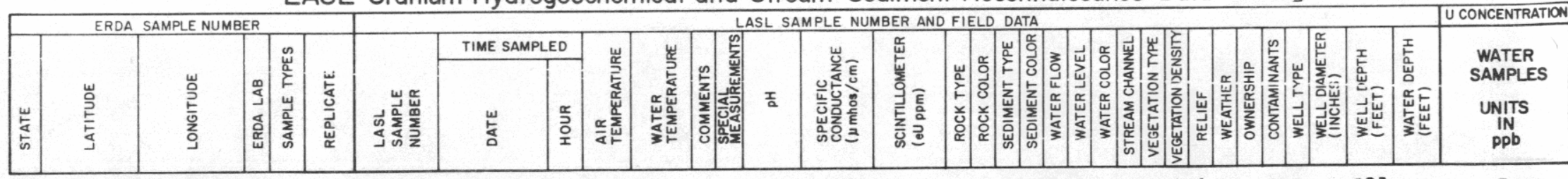

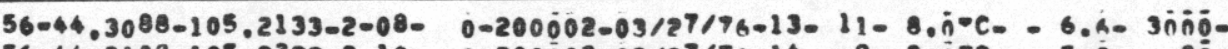
$56-44.3108-105.2322-2-10-0-200003-0319717 n-14-9-9.0-C-0$ - 5.2- 0 - 0 $56-44.3216-105.2044-2-08-$ $56-44.3480-105,1438-2-08-$
$56-44.3158-105,1450-2-109-$ $56-44.31322-105,1861-2-09=$ $56-44.3630-105,1980-2=08$ $56-44.3827-105.1947-2-08-$
$56-44.3986-105.2283-2 \times 06=$ $56=44.3986-105 \cdot 2283-2=06=$ $56-44.4405-105.2261-2-10-$ $56-44.3458-105.1122-2-10-$
$56-44,3911-105.1022-2-10-$ $56-44.3911-105,1022-2-16$. $56-44.4325-105.0886-2-108$
$56-44.469-105.0769-2-08$ $56-44.4591-105.0566-2-68$ $56=44.4800-105.0219-2-08-$ $56-44.3355-105 \cdot 0516-2-080$ $56-44 \cdot 3894-105.0561-2-10=$
$58-4 ., 3847-105.0211-2-08=$ $56.44 .3891-105,0061-2-10$ $56-44.4369-105.0068-2-10-$
$56-44.3497-105.0111-2-06-$ $56-44,3141-105,0088-2-08$ $56-44.2744-105.0741-2-0 \overline{8}-$ $56-4 . .2305-105.0453-2-08$ $56-44.2344-105.0105-2-08$ $56-44.2300-105 \cdot 1194-2-09-$ $56-44.1916-105.0666-2-08$ $56-44.2444-105,2125-2-08$ $56=4.2222-105,2136-2-09=$ $56-44.7405-105,7083-2=06$ $56-44.7280-105,7016-2-09$ $56-44.7216-105.6638-2=06$ $56-44,2913-105,1672-2-08$ $56=44.2902-105.1516-2-10-2$ $56-44.3050-105 \cdot 3616-2=6$ $56-44 \cdot 3188-10^{5}, 3^{5} 16-2-0$ $56=44.3472-105.3705-2-00$ $56-44.3472-105 \cdot 3461-2=10$ $56=44.3216-105.3252-2-06$ $56-44.3319-105 \cdot 3213-2-08$ $56-44.3536-105 \cdot 309 i-2-06$ $56-44.3669-105 \cdot 3494-2-10-$ $56-44.4208-105.2547-2-08$ $56-44.4411-105.2966-2-10=$ $56-44.5544-105.6666-2-08$ $56-44.2736-105.6522-2-08-$ $56-44.2700-105.6327-2-08$
$58-4$. $56-44.2763-105.7069-2-08$
作

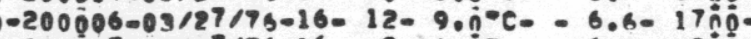
$0-200007-03 / 27 / 76-16-9-14.0-2-6.3-900-$

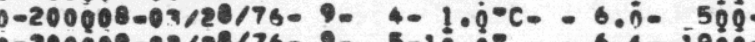
$0-200009-03720176090-5-100 n-0.60-19 n 0$ $0-200010-03 / 20 / 7 b-10-5-6.0-2.6-29 n 9-$ $0-200911-03 / 26 / 96-1109-7.00:-6.6024000$

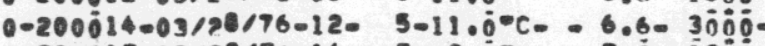
$0-200117-03 / 20 / 76-14-5-6.0=-5.201800=$ $0-200618003 / 26 / 76-15.509 .0^{\circ}-0.3013000$ $0-200019-03 / 26 / 76-16-5-6.00-6.30300-30$ $0-200020-03 / 96 / 76-16-5-9.0-C-2.0-4300-$ $0-260021-03 / 2917 \theta-16-5-6.0-C-6.60600-60$

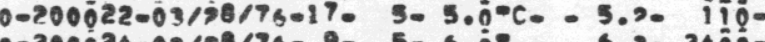
$0-200024-03 / 23 / 7409-5-6.0^{\circ}-0.6034000$

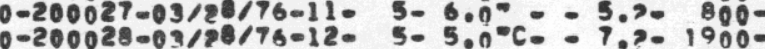
$0-200029-03 / 98 / 76-1205-5.0^{\circ}-5.7-120$ $-200031-03 / 82 / 74-1405-60.0=5.50360$

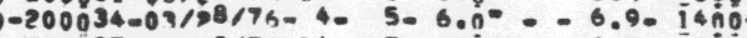
$0-200035-03 / 28 / 76-14-5-6.00=-6.9-1600$ $0-200037-03 / 30 / 7 B=-80-8.0-2.4-1300=$ $0-200040=03 / 30 / 76-10=7-4.0-C-C .6-3600-$ $0-200041003 / 30 / 76-10$ - 7-6.0" - - 6.6- $2100-$ $0-200042-03 / 30 / 75-12-7-7.0--5.5-130-$ $0-200044-03 / 30 / 76-13-7-8.0^{-C}-C_{-}=6.6-2300-$ $0-200045-03 / 30 / 76-12-3-0.0-30-6.3-30$ $0-200946-03 / 30 / 76-13-8-8.0-0_{-}-5.5-99-$ $0-200047-03 / 31 / 76-10-13-7 \cdot 0=\mathrm{C}-6 . h-999-$ $0-200048-03 / 31 / 76-10-13-9 \cdot 0-0-6.9-1800-$

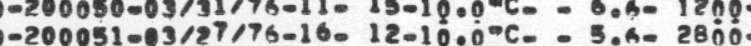
$0-200032-03 / 27 / 76-19.909 .0^{-0}-5.9-2830-$

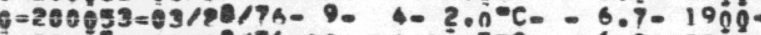
$0-200054-03 / 28 / 76-100 \quad 4-10.5-C_{-}-6.8-2200$ -200055-03/88/76-11- 3- 6.2-C- - 7.5- i4ho$0-200056-03 / 28 / 76-120-2-7.5=0.1-0.1090$

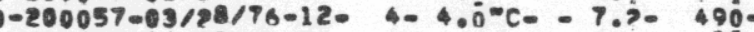

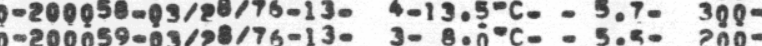
$0-200059-03 / 28 / 76-130-3=8$ $0-200060-03 / 28 / 76-1403-7 \cdot 0-0.5 .5-500-50$

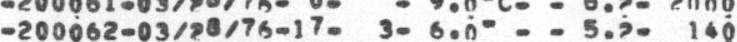
$0-200064-03 / 30 / 76-10=70.0^{\circ} 0^{\circ}=-6.60740-$ $0-200065-04 / 75 / 76-8-12-8.0^{\circ}-=6.9-1200^{\circ}-$

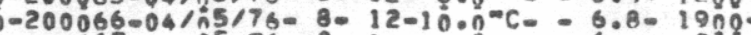

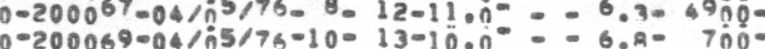

$9-1-7-4-6-2-3-1--4-3-2-2--1-3-a-250-$ $6-1-7-5-6-1-3-3-6-4-3-2-2--9-1$

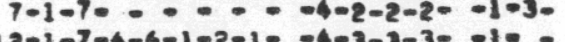
$12-1-7-4-6-1-2-1<-4-3-3-3--1-$ $6-1-6-4-6-1-2-10-4-9-2-2--1-3$

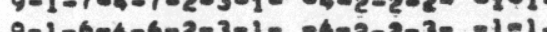
$9-1-604-602-3-1=04-2-2-30-1-10$

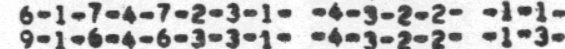
$9-1-7-4-7-1-3-2--4-3-2-3-3-9-$ $\begin{aligned} & 6-1-6-4-6-1-2-2-4-9-2-2-10 \\ & 6-1-6-4-6-1-2-20\end{aligned}-4-3-2-2-10=$ $9-1-7-4-7-2-3-5--4-3-2-3-1-1-$ $9-1-7-4-7-1-2-20-4-3-2-2--1-1$ $9-1-7-4-7-1-2-2=-4-3-2-2--1-10$ $16-1-7-4-7-1-2-1=-4-3-3-2--1-3-$ $12-1-7-4-7-2-3-10-4-2-3-1=-1-4$ $12-1-6-4-6-1-3-1<-4-3-3-2--1=-$
$6-1-7-4-7-1-2-1<-4-2-3-3-1-4=$ $9-1-6-4-6-1-3-2-04-2-3-3--10$ $12-1-6-4-6-1-3-2-6-3-3-3-3-3-10$ $9-1-6-4-6-2-3-1--4-2-3-3--1-4-$ $2-1-6-4-6-5-3-1=-4-3-5-1=-1-1=$ $12-1-6-4-6-1-2-1--3-2-4-2--1=4-$ $11-1-7-4-7-3-3-1=-4-3-4-2=-1=3$ $9-1-6-4-6-1-2-4<-4-3-2-2--1=$
$8-1-6-4-6-1-2-1-04-3-2-2-2-1-3=$ $16-1-6-4-6-2-2-1<-3-3-2-1--1-3-$ $9-1-7-4-7-1-2-2--4-3-2-1--1-1-$ $9-1-6-4-6-3-3-10-4-3-2-1--1-1$ $6-1-6-4-6-1-3-2-04-4-2-1--3-1-$ $12-1-6-4-6-2-3-1-1-4-9-2-1--1-1-$
$6-1-7-4-7-2-3-1--4-3-2-3--1-3-$ $9-1-7-4-7-2-3-1=-4-3-1-3--1=$ $16-1-70=-20-0-3-2-3--1-3-$ $12-1-70 .-0-4-3-2-3-1-1-5-6-210=$ $10-1-6-\cdots-2-4-2-3-3-1-3-3-60$ $15-1-7-5-2-1-3-2--4-2-2-5-10-10$
$9-1-70-0-20-10-4-3-3-3--10$

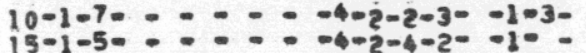
年 $10-1-7-5-8-2-3-2--4-3-3-2--1-1-6-200$

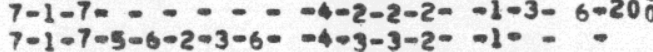
$15-1-7-\ldots-0-4-2-2-1--1-3-60$ $10-1-6-4-6-3-3-1--4-3-2-1=-1-1-$
$6-1-6-6-6-3-3-10-4-3-2-1=-1-8-$ $10-1-7-4-7-3-3-10-4-3-2-10-1-1=$ $10-1-7-4-7-3-3-10-4-3-2-1-1-1-1=$
$13-1-6-4-6-3-3-1-4-3-2-1--1-1=$
5.55 0.61 0.04 0.16 1.68 1.03 0.7 1.71 3.10 2.29 0.10 0.10 4.44 0.33 0.32 2.17 0.68 0.47 0.82 4.42 8.21 9.40 1.22 0.10 0.86 0.10 0.10 1.46 0.10 4.31
0.91 0.52 1.53 1.40 0.45 1.63 0.70 0.65 0.97 6.16 0.35 0.10 0.52 0.28 0.10 
LASL Uranium Hydrogeochemical and Stream Sediment Reconnaissance Data Listing

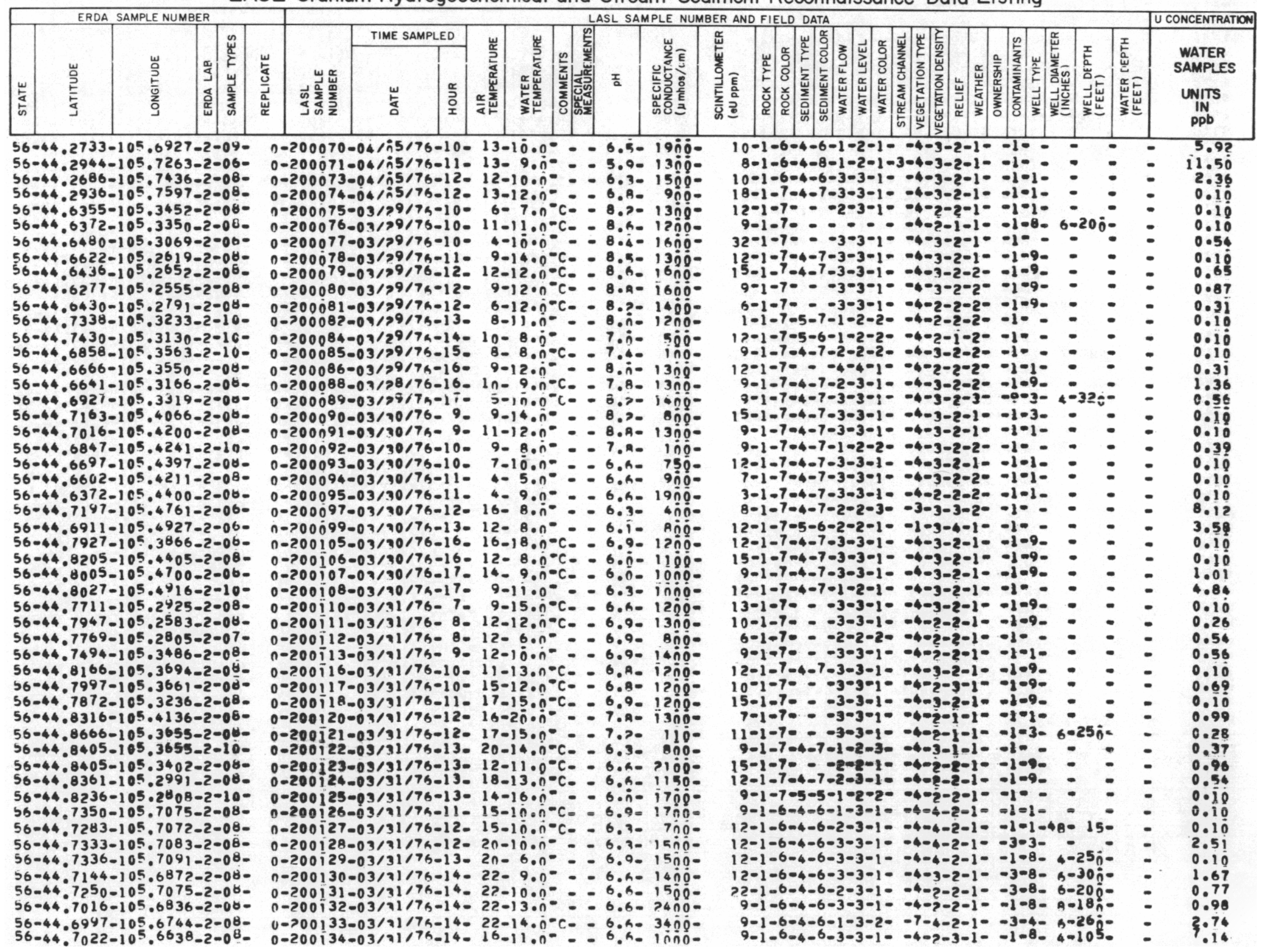


LASL Uranium Hydrogeochemical and Stream Sediment Reconnaissance Data Listing

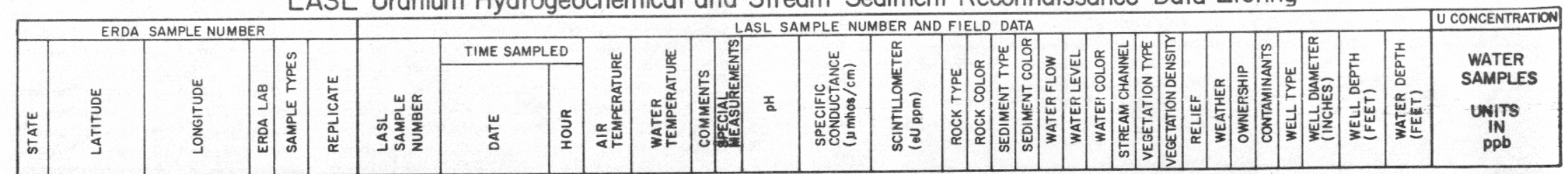

$56-44.6980-105.6955-2-66-0-200135-03 / 31 / 76-14-15-10.0-0-6.3-900-$ $56-44.6811-105.7116-2-08-0-200136-03 / 91 / 76-15-14-1900^{-C} \mathrm{C}-0.6-9-1100-$ $56-44.6747-105.6888-2-04-0-200137-03 / 31 / 76-17-15-10.0=-6.3-2500-$ $56-44.7650-105.6944-2-08-0-200139-04 / 11 / 7 h-9-18-9.00^{\circ}-0.4-1300-$

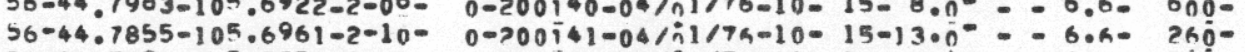

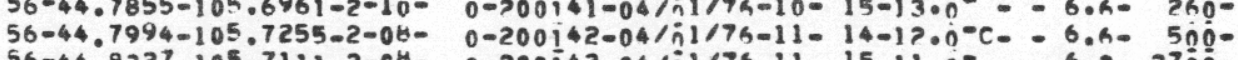

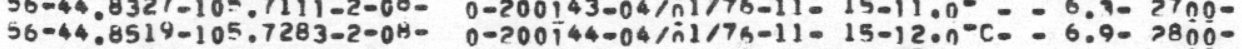
$56-44.8338-105.8100-2-08-0-200147-04 / n 11 / 79-13-21-10.00-0.90-2500-$ $36-44.8227-105.2666-2-08$ - 0-200151-03/71/7a-14- 14-11.n- - - 6.4- $12000-$ $56-44.8519-105.2680-2-08-0-200152-03 / 91 / 76-14-17-16 \cdot n-0-6.9-12 n 0-$ $56-44.7594-105.5077-2-10-0-200157-04 / 12 / 76-10-0-500-\mathrm{C}-0.3-1200-$ $56-44.7769-105.5550-2-06-0-200158-04 / 32 / 75-0-0-5 \cdot n-$ - $-6.4-1100-$

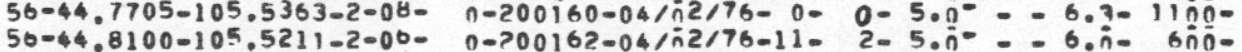
$56-44.8386-105.5416-2-08-0-200166-04 / i 2 / 76-12=2-4.0^{-}=-t_{0}=15=150^{\circ}$ $56-44.6905-105.5641-2-08-0-200169-04 / 72 / 76-14-2-8.00^{-}-0.6-69000-$ $56-44.7027-105.5472-2-08-0-200170-04 / h_{2} / 7 h-15-1-8 \cdot n^{-}=-7 . ?-2000-$

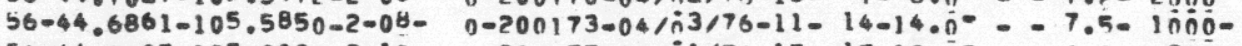

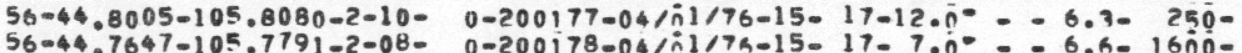

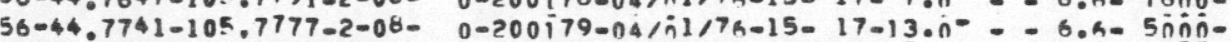
$56-44.7627-105.7625-2-08-0-200180-04 / 01 / 76-16-13-10.00^{-C}-C_{-}-6.7-3200-$ $56-44.7544-105.7244-2-08-0-200181-04 / 11 / 7 h-16-13-11,0=-0.4-45 n n-$

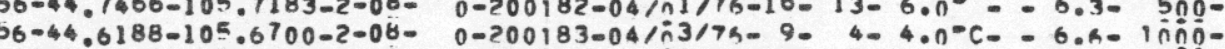
b6-44.6802-105.6494-2-08- 0-200i85-04/i3/76-11- 7-4.0-C- - G.A- $1200-$

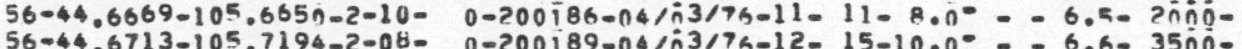

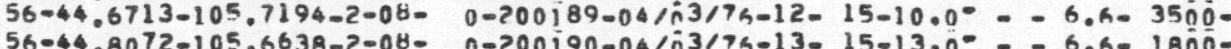

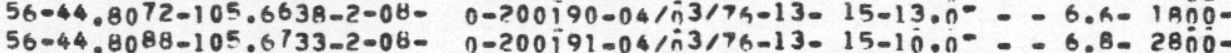
$56-44.8347-105.6333-2-10-0-200192-04 / 033 / 74-14-10-12 . n^{-}-0.1-690-$

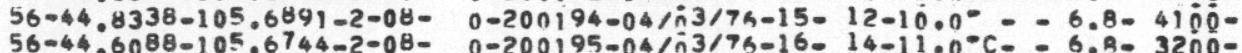
$56-44.6077-105.6713-2-08-0-200196-04 / 03776-16-14-11.0=-6.8-3500$ $56-44.5997-105.6450-2-06-0-00700197-04 / n 3 / 76-16-14-9.01-=-6.3-1400-$ $56-44.5808-105.6686-2-08-0-200198-04 / 73 / 76-17-15-12.0^{-0} \mathrm{C}-\mathrm{C}_{-6.8-1700-}$

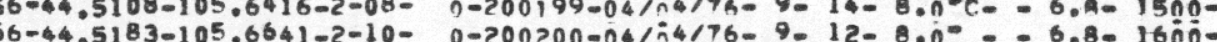
$56-44.7294-105.5733-2-08-0-200202-04 / i 3 / 76-12-13-10.00-07.5-1000-$ 56-44. $9016-105.5397-2-08-0-200207-04 / 33 / 76-14-11-14 \cdot 0^{\circ}-0.6-2800-$

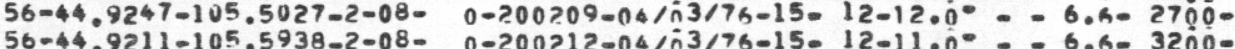
$36-44.9413-105.6127-2-08-0-200214-04 / 33 / 76-16-13-13.0-C=-6.6-3500-$ $56-44.9916-105.5911-2-10-0-200217-04 / 03 / 76-16-14-11.0-\mathrm{C}-0.6 .3-200-$ $56-44.9461-105.4922-2-16-0-200221-04 / 76 / 75-9-14-10.00=-6 . \dot{n}-1200=$

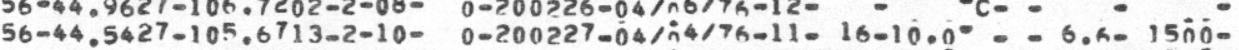
$56-44.5372-105-7208-2-16-0-200228-04 / 84 / 7 a-11-18-13.00=-6.3-170-$
$12-1-6-4-6-3-3-1--4-3-2-1--1-8-6-90-$ $12-1-6-4-6-1-3-1=-4-3-2-1--1-4-6-443-$ $9-1-6-4-6-3-3-1--4-3-3-1=-1-8-6-1330$ $9-1-6-4-6-2-3-1--4-2-3-1--1=-:-$ $16-1-6-4-6-1-3-2-1-4-2-3-1--1=1-$ $12-1-6-4-6-1-2-3--4-2-4-1--1-1-$
$9-1-6-4-6-2-2-1-0-4-2-4-1-=1-3-$ $9-1-6-4-6-2-2-1=-4-2-4-1=-1-3-$
$6-1-6-4-6-1-2-1=-4-2-2-1--1-10$ $9-1-6-4-6-3-2-1--1-2-4-1--1-3-$ $10-1-7-0-2-2-1--4-2-2-1--1-1-$. $15-1-7-5-6-2-2-1--4-2-2-1=-1-1-$

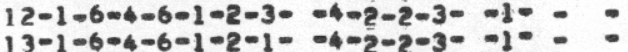
$13-1-6-4-6-1-2-1-4-2-2-3-1-1-0$.

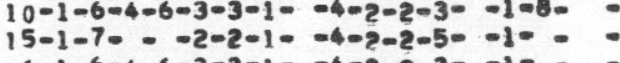
$\leqslant-1-6-4-6-2-2-1--4-3-2-3--1-$ $12-1-6-4-6-3-3-1=-4-3-2-3--1-3=$ $12-1-6-4-6-3-3-1--6-3-2-3--1-1-$
$20-1-7--3-3-1--4-2-2-1--1-1-$ $12-1-6-4-6-1-2-3--4-3-2-2--1=-$ $17-1-6-4-6-3-3-1=-4-2-4-3--1-3-$ $12-1-6-4-6-2-3-1--4-2-4-3--1-3-$
$16-1-6-4-6-2-3-1--4-2-3-3--1-3-$ $16-1-6-4-6-2-3-1--4-2-3-3--1-3-$
$6-1-6-4-6-3-3-1--4-2-3-3--1-3-$ $6-1-6-4-6-3-3-1--4-2-3-3--1-3-$
$9-1-6-4-6-3-3-1--4-3-3-3--1-3-$ $12-1-6-4-6-1-3-1--4-3-3-1--1-1-6-18 j$ $6-1=6-4-6-1-2-3--4-4-1-1--1-1-6-180$ $6-1-7-4-7-1-2-2--4-3-2-1=-1-1-$
$8-1-6-4-6-3-3-1--4-3-1-1=-1-1-60-130-$ $2-1-6-4-6-3-3-1--4-3-2-1--1-4-$. $12-1-6-4-6-3-3-1--4-3-2-1--1-3-$. $9-1-6-4-6-1-2-2-3-4-2-3-1--1-0$ $12-1-7-4-7-3-3-1--4-3-2-1--1-3-6-27$
$6-1-6-4-6-3-3-1-4-3-2-1=-1-3-6-275$ $6-1-6-4-6-3-3-1=-4-3-2-1=-1-3-6-4400$. $19-1-6-4-6-3-3-1--4-3-2-1--1-1<=$ $16-1-6-4-6-1-2-2-4-4-2-2-1=-1-1=$
$0-1-6-4-6-1-2-1-0-3-2-1--2+10$ $7-1-7-4-7-1-2-1--4-3-2-1=-3=$ $13-1-7--2-2-1--4-2-2-1--1-1-$ $12-1-7--3-3-1--4-2-2-1--1-3-6-3000$ 15-1-7-- - 3-3-1- $-4-2-2-1--1-1-=$ $15-1-7--3-3-1--4-2-2-1=-1-1-$

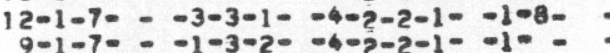
$6-1-6-4-6-1-2-4--4-2-2-2--1-$ $16-1-6-4-6-1-2-1--4-3-2-1--1=-$ $16-1-6-4-6-1-3-3--4-3-2-1--10=$ $9-1-7-4-7-1-2-1--4-3-2-1--1=1-$
1.77

0.10

3.36

0.28

1.14

0.51

5.04

6.04

3.13

0.10

0.16

0.10

3.96

2.20

1.73

3.21

0.52

0.68

0.32

1.20

6.31

3.41

2.74

1.14

0.10

8.06

5.73

0.91

4.58

2.69

1.44

0.51

2.40

0.24

0.10

.43

0.10

1.67

0.36

0.18

7.60

0.10

0.24

1.81

1.86

0.38

0.76 
LASL Uranium Hydrogeochemical and Stream Sediment Reconnaissance Data Listing

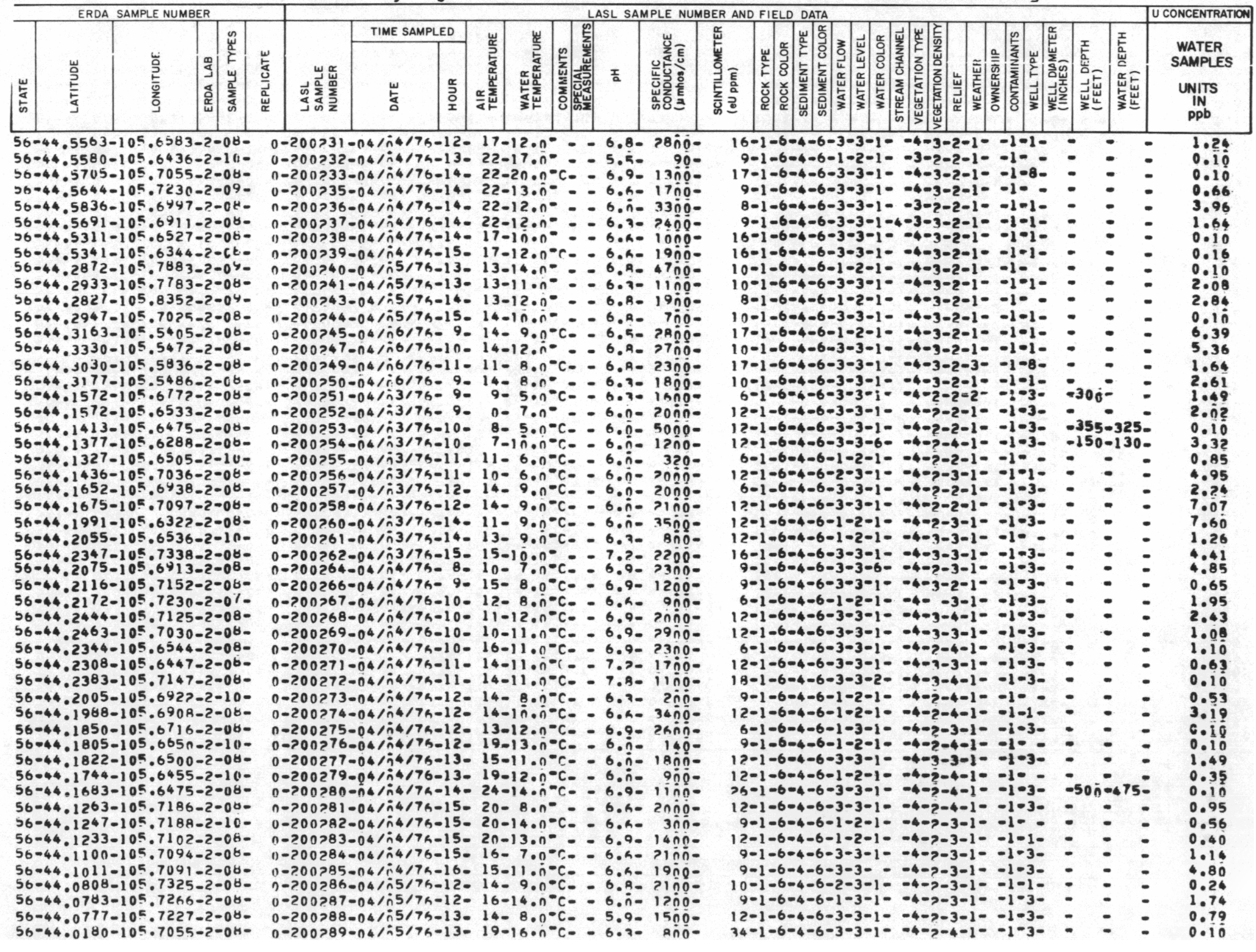




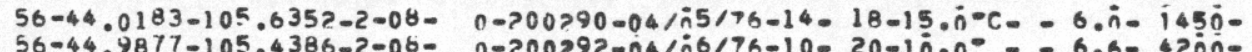

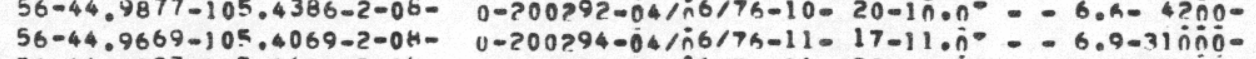
$56-44.9827-105.3661-2-08-0-200296-04 / 86 / 7 A-11-22-16.0-C-0-6.9-1100-$ $56-44.9780-105 \cdot 3144-?-08-\quad 0=200299-04 / 16 / 76-12-22-14 \cdot 0-C-6-6 \cdot 9-1 n n 0-$

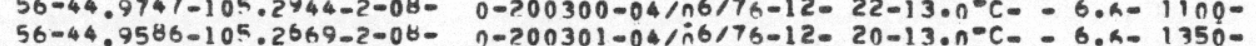
$56-44.9266-105.3561-2-0 H-0-200302-04 / n 6 / 7 h-12-19-18 \cdot n-C-0-6.9-1 n i 0-$

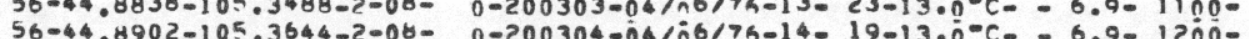
$56-44.8902-10503644-2-08-0-200304-04 / 26 / 76-14-13-1300^{-}-C_{-}-6.9-12 n 0-$

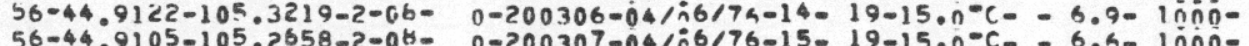
56-44.9105-105.2658-2-08- 0-200307-04/r6/76-15-19-15.n-C- - 6.4- $10 \Omega 0-$

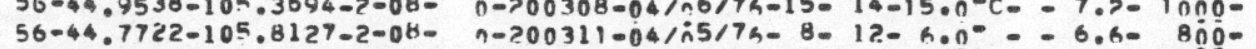

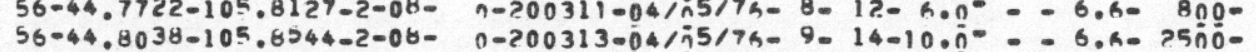

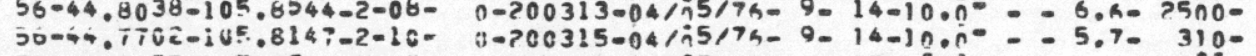
56-44.7255-105.7772-2-04- 0-200316-04/R5/7h-11- 15-10.00- - - G.R- Año$56-44.7183-105.7661-2-08-0-200317-03 / n 5 / 7 a-11-15-9 \cdot 0^{\circ}=-6.9-900-$ $56-44.7116-105.7930-2-06-0-200319-04 / n 5 / 76-13-21-10.0-0.60-90-2000-$

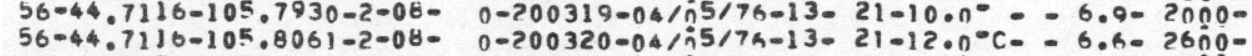
$56-44.7372-105.8094-2-08-0-200321-04 / 75 / 76-13-22-11 \cdot 0_{-}-0.3-190_{0-}$

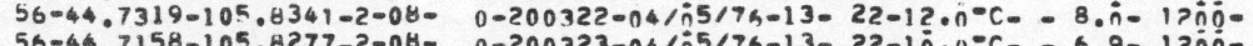
$56-44.7158-105.8277-2-08-\quad 0-200323-04 / 55 / 7 A-13-22-1 n_{0} \cdot 0^{\circ} C_{-}-6.9-1300-$ $56-44.7075-105.7755-2-08-0-200324-04 / n 5 / 76-13-22-9 \cdot 0=-6.6-3 n n 0-$ $56-44.6833-105.8347-2-08-0-200325-04 / 26775-9-18-12 \cdot 0=-8.5-2900-$

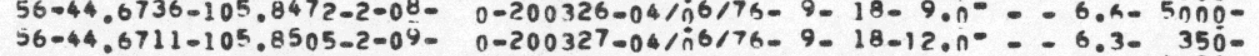
$36-44.6544-105.847 ?-2-04-0-200328-04 / 76 / 75-10-17-9.0^{\circ} \mathrm{C}-0-6.6-1600-$ $56-44.6827-105.8663-2-04-0-200329-04 / 76 / 7 k-10-17-14.0-c_{-}-6.9-1 ?_{0} 0-$

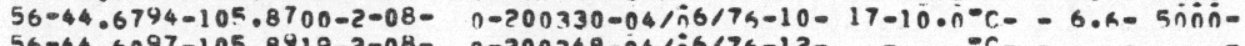
$56-44.6097-105.8919-2-08-$ $56-44.9200-105.4183-2-08-$
$56-44.9177-105.4450-2-08-$ $56=44.9258-105.4733-2-08-$ $56-44.9841-105.7044-2-08-$ $56-44.9916-105.6972-2-08-$ $56-44.8877-105.7100-2-10-$ $56-44.9016-105.7191-2-08-$
$56-44.9086-105.7358-2-08-$ $56-44.9086-105,7358-2-08-$
$56-44.3150-105.4463-2-08-$ $56-44.3411-105 \cdot 4305-2-08=$ $36=44,3630-105 \cdot 3833-?-08-$ $56-44.3127-105.4880-2-06-$ $56-44.3241-105.4283-2-06-$ $56=44.3616-105.4069-2-08-$ $56-44.2972-105,4444-2-08-$ $56-44.2566-105.5408-2-06-$ $56-44 \cdot 3244-105.7063-2-10-$ $56-44.3194-105.8016-2-10=$
$56-44.3558-105.7966-2-96-$

$0-200351-04 / \hat{n} 6 / 7$ B -15 - 14-13. $0-C_{-}=6.3-4100-$ $0-200353-04 / 26 / 75-16-13-12 . n^{-}-7_{0}-8$ - $800-$

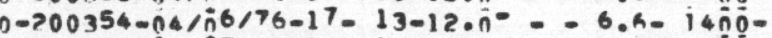
$0-200359-04 / 07 / 7 a-10-14-13.0^{\circ} \mathrm{C}=-7.201400-$

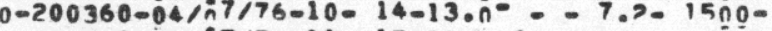
$0-200363-04 / n 7 / 76-11$ - $15-13 . n^{-C} C_{-}-6.9-600-$ $0-200364-04 / 0^{7} / 76-11-15-13 \cdot 0^{-} \mathrm{C}-{ }_{-}-7.2-2900-$ $0-200365-04 / A^{7} / 76-11-16-14 \cdot n^{-1} C_{-}-7.5-1800-$ $0-200367-04 / 10 / 76-12-$ $0-200368-04 / n 6 / 76-12$. $0-200369-04 / \mathrm{n} 6 / 7 \mathrm{~h}-12$ $0=700370-14 / 26 / 76-12$. $0-200372-04 / 66 / 76-12-$ $0-200375-04 / 26 / 75-15-$ $-\mathrm{C}-=$
$-\mathrm{C}-\mathrm{-}-$

$-c_{-}=$

$-c_{-}=$

c.:

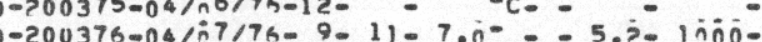

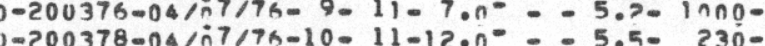
$0-200379-04 / 57756-10-11-12 \cdot n^{\circ}-5.5-230-$ $n=200381-04 / n 7 / 7 n-12=14-11,0 n=:-5.9-3200-$ $12-1-6-4-6-3-3-1--4-2-2-1--1-3-\div:=0.16$ $12-1-6-4-6-1-3-1--4-3-2-2--1-2-$ $9-1-6-4-6-1-2-1--4-3-2-2--1-2-\div \quad-\quad 7.42$ $9-1-6-4-6-4-3-1=-4-3-2-2-1-1-0.77$ $0-1-6-4-6-3-3-1=-2-3-2-2--1-9-2=0.45$ $9-1-7-4-7-3-3-1--4-3-2-2--1-9-=$ $15-1-7-4-7-3-3-1--4-3-2-2--1-9-\quad-\quad-0.10$ $12-1-7-4-7-2-3-1--4-3-2-3--1-9-:=0: 0.10$ $12-1-7-4-7-2-3-1--4-3-2-3--1-9-\div-0.22$ $9-1-7-4-7-3-3-1=-4-3-2-3--1=9-\infty-0.22$ 0 $10-1-6-4-6-3-3-1--4-5-3-1--1-3-0.10$ $16-1-604-603-3-1=-402-3-1-10-103-0.10$ $16-1-6-4-6-2-3-1--4-3-3-1--1-1=0.5$ $12-1-6-4-6-3-3-1--4-2-3-1--3-2-6-360-$ - 0.10 $16-1-6-4-6-3-3-1--4-2-3-1--1-1-6-190-0.10$ 作 $16-1-6-4-6-3-3-1-04-2-3-1--3-2-\div \quad 2.40$ $9-1-7-4-7-1-2-2-04-3-2-1--3-1-0=30$ $10-1-6-4-6-2-3-1--4-3-2-1-0-3-1=0$ $15-1-7-4-7-3-3-1=-3-3-3-1-09-3-0.5$ $9-1-6-4-6-3-3-1=-4-2-2-1-0.3-3-0$ $9-1-6-4-6-3-3-1--4-3-3-1--1-1-3-\div:=2.16$ $16-1-4-4-6-2-3-1--4-2-3-2--1-1-\div-0.29$ $16-1-4-4-6-1-2-3--4-2-3-3--1--\ldots+1.5$ $9-1-6-4-6-1-2-1--4-2-3-3--1-1-\div=0.77$ $12-1-6-4-6-2-3-1-04-3-3-3-0.63$ $9-1-7-4-7-1-2-1--4-3-3-3--1-1-\div ;=9.82$ $9-1-7-4-7-0-4-2-2-3--1-9-10.41$

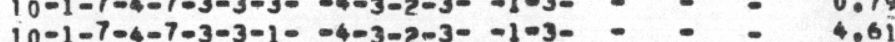
$10-1-7-4-7-3-3-1--4-3-2-3--1-3-\div:=4.61$

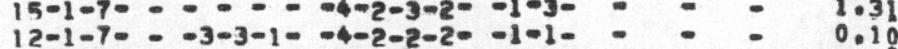
$9-1-7--1-2-3--4-2-2-1--10-0-0.40$ $15-1-70--3-3-1-4-4-2-2-1--1=3-6-30 j 0 ; 0.21$ $15-1-70-0.10$

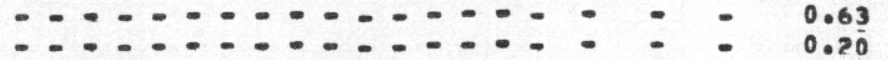

:......... - $=1.74$

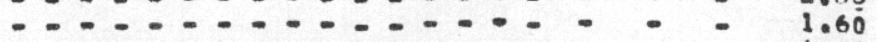

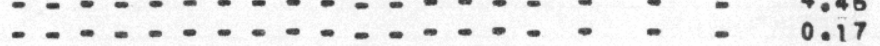
$10-1-6-4-6-3-3-1--4-3-2-2--1-1-=-\quad=1.36$

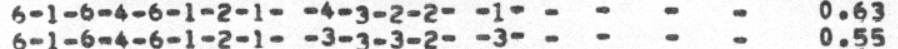
$6-1-6-4-6-1-2-1--3-3-3-2--3-1$
$10-1-6-4-6-3-3-1--4-3-2-2--1-1-$ 
LASL Uranium Hydrogeochemical and Stream Sediment Reconnaissance Data Listing

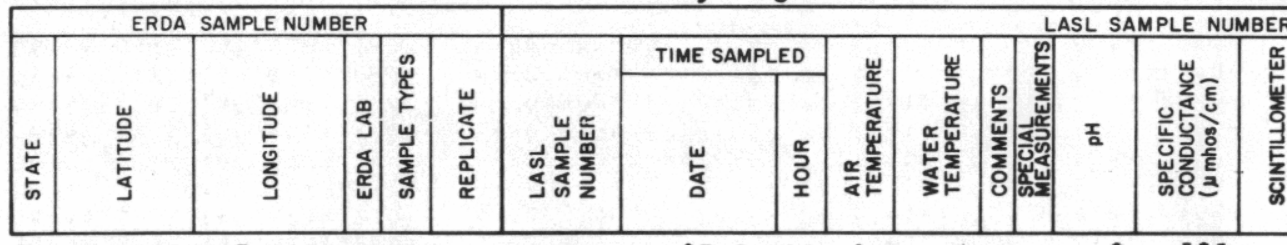

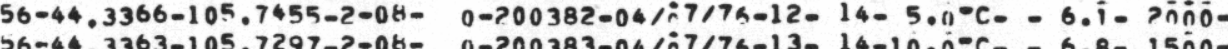

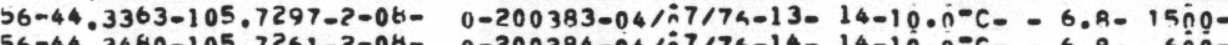

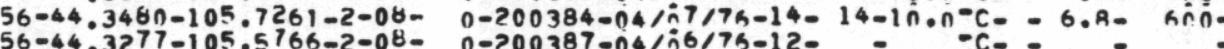
(1) $56-44.2794-105.4288-5-060$ $56-44.2794-105.4288-2-06$ $36-44.2419-104.6755-2-08$ $56-44.2072-106,6316-2-07-$ $56-44.2072-106.6316-2-07$ $56-44.1200-106.5825-2-06$ $56-04.1277-106,5075-2-06-$ $56-44.1594-1065961-5-080$

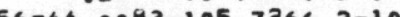
$56=44.0003-105.7266-2-10-$ $56-44.0619-105.7216-2-06$ $56-44.0713-105,7233-2-08-$ $56-44.0521-105.6883-2-08-$ $56-44.0663-105.7105-2-10-$ $56-44.0861-105.6125-2-08$ $56-44.0861-105.6222-2-04-$ $56-44.0655-105.5880-2-06$ $56-44.0566-105.5283-2=06$ $56-44.0672-105.5005-2=0$ H $56-44.0994-105.5916-2-0 \mathrm{H}$ $56-44.0877-105.5722-2-0 \%$ $56-44.0877-105.5722-2-0$
$56-44.0947-105.5452-2-08$ $56-44.1341-105.5472-2-08$ $56-44,1444-105.5250-2-06-$ $56-44.1444-105.5250-2-08-2$ $56-44.9655-106.2677-2-080$ $56-44.9930-106.3202-2-08$ $56-44.9425-106.4961-2-08-$ $56-44.9425-106.4961-2-08$
$56-44.6230-105.2944-2-08$ $56-44.5994-105.2577-2-08-$ $56-44.5994-105 \cdot 2577-2-08-$
$56-44.5713-105.2658-2-06-2$ $56-44.5713-105 \cdot 2658-2-06-$ $56-44.3758-105.4583-2-06-3933-105,3902-2-04-2$ $56-44.3933-105.3902-2-08-$
$56-44.4019-105.3613-2-06-$ $56-44.4430-105.3847-2-06=$ $56-44.4555-105 \cdot 4100-2-06$ $56-44.4241-105 \cdot 3$ 358-2-08 $26-44.4580-105 \cdot 3327-2-06-$ $56-44.4127-105 \cdot 3105-2-06-$ $56-44.5491-105.5655-2-06$ $56-44.5283-105.5461-2-08$ $56-44.6800-106.1311-2=08$ $36-44.7183-106.2677-2-08$ $56-44.6783-106.3666-2-08-$
$56-44.7130-106,3336-2-08-$ $0=200387004$ in $n \rightarrow 00389-04 / \pi 6 / 76-12-\quad-C_{-}^{-C}-C_{0}^{-}$

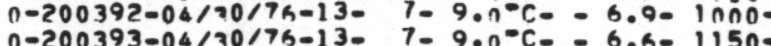
$0=200393-0420175-13-1150-$ n-200396-04/30/74-14- $11-100.5-c=6.9-3950-$ $0-200396-04 / 20174-14-11-10.5-c-6 \cdot 3-1300-$ $0-200398-04 / 3017 n-15-11-10=0-c=0.9-17 n 0-$ $0 \rightarrow 200399-04 / 20176-16-12-9.5-c=-6=0$

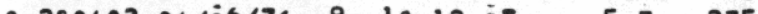

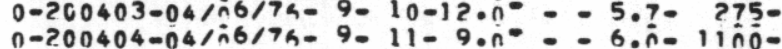
$0-200405-04 / 16 / 7 h-9.12-16.0-C_{-}=6.4-1500-$

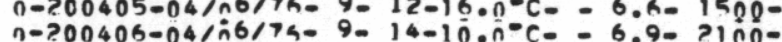
$0-200408-04 / j 6 / 75-10-16-10 \cdot n^{-C} C_{-}-6.9=2250-$ $0-200409-04 / n 6 / 74-11-13-1 n \cdot n-$ - 6.a- 390$0-200420-04 / 26 / 76-12-$ $0-200421-04 /$ h6/7k-12. $0=700423-04 / 26 / 76-12$ $0=200424004 / 36175-120$ $0-200426-04 / 46 / 7 k-12$. $0-200427-04 / 26 / 76-12$ 0 $n=? 00430-04 / n 6 / 7 h-12$. $0-200431=04 / 66 / 76-12=$

$0-200435-04 / \mathrm{n} 6 / 75-$ - 0 - 0 -

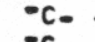

-C=-

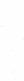
$0-200436-04 / 47175-14-14-9 . n=-6.3033 n 0-$ $0-200440-04 / 20 / 7 h-10-17-12 \cdot 0^{-}-6 . \hat{n}-250-$ 0-200443-04/20/76-12- $17-1100$ - - 6.h- >7no$0-200450-04 / 30 / 76-14-20-15.0^{-}-6.9-240-$ $0=700451-0412176-16-27-18.0-C=6.9-15 n 0-$ $0-200452-04 / 12 / 7 h-16-28-19.0^{\circ} \mathrm{C}-6.8-1550-$ $0-200453-04 / 1274-16-27-18 . n^{-}-0.9-13 n O-$ $0-200455-04 / 13176-8-11-13.0^{-C}-C_{-}-6.9-160-$

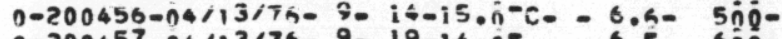
$0-200457-04131760$ - 19.14.0 - 6.5. 6no$0-200458-04 / 13 / 75-9-20-15.0^{\circ}-0.8-8 \mathrm{nij}-$ $0-200459-04113176-9-20-1200-0.10175$ $0-200460-04 / 13 / 76=9-2 n-13.0=-6.303100-$ $n \rightarrow>00461-04 / 13 / 7 a-10=20-15 \cdot 0^{-}-6.6-20 n 0^{-}$ $n=200464-n 4 / 13 / 76-11-21-11 \cdot n=-6 \cdot n-360-$ $n-200469-04 / 13 / 74-15-22-15 . n^{-}-7.2-2300-$ $0-200470-04 / 13 / 79-15-21-14 . n--5.6-170-$ n-200473-04/477n-9- 12-16.n- - 7.2- $12 n 0-$ 0-200475-04/i4/7G-10- 13-13.0-C- - G.4- 15ño$n-200480-n 4 / i 4 / 7 a-11-16-11 \cdot n-C-E=6 . R-1100-$
$0-200486-04 / i 4 / 74-12-16-16 \cdot 0-C=-6.8-21 n 0-$

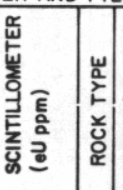

$10-1-6-4-6-1-2-1--4-3-2-2--1-1-$ $6-1-6-4-6-1-2-1--4-3-3-2--1-1=$ $10-1-6-4-6-1-2-1=-4-3-3-2--1-1=$

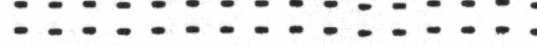
$\ldots \ldots \ldots$ $8-1-7-2=-1--4-3-2-1--1-3-6-24 j=$ $9-1-7-5-7-2-2-1-2-4-3-2-1--1-1-$ $8-1-7-5-7-2-2-1-2-4-3-2-1--1<-$

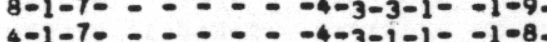

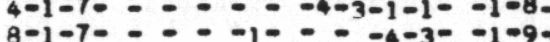
$8-1-70-0-1=-4-3-3-1=-1-9-$ $18-1-6-4-6-1-2-1--4-2-2-3--1--$ $6-1-6-4-6-3-3-1--4-2-3-2--1-3-$ $6-1-6-4-6-3-3-1=-4-2-3-2--1-3-$
$15-1-6-4-6-2-3-1=-4-2-2-2--1-3-$ $12-1-6-4-6-1-3=3=-4-2-3-3--1-3-$ $9-1-6-4-6-1-2-2--4-2-3-3--10$

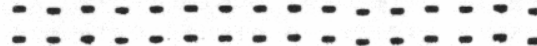
-. - . - . - - - - - -

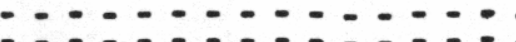

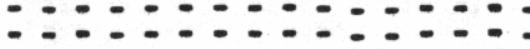

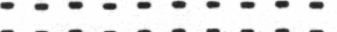

-

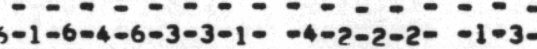
$12-1-7-5-7-2-2-1--4-2-3-1--1-1-$

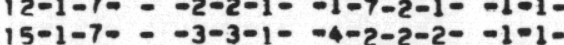

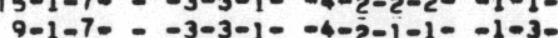
$9-1-7-0-3-3-1--4-2-1-1--1-8$ $9-1-7-1-1-3-4-2-1-1--1-1-$
$9-1-6-4-6-2-3-3-4-3-3-2-9-9-1-$ $12-1-6-4-6-2-2-3-=4=3-2-2--1-9-$ $12-1-6-4-6-2-3-1=-4-3-3-2=-1-$ $6-1-6-4-8-2-2-1-4-3-3-1--1-$
$2-1-6-4-6-2-2-1-0-4-3-3-1--1-$ $18=1=6-4-6=3-3=1=-4-3-2-1=-1=1$ $2-1-7-4-7-3-3-1--1-3-3-5-1-3-3$ $9-1-6-4-6-3-3-1--4-3-3-2--1-1-$
$9-1-6-4-6-2-3-1--4-3-2-2--1-3-$ $9-1-6-4-6-3-3-1--4-3-2-2--1-3-$ $9-1-7--2-3-1=-4-2-2-3--1-1-$
$15-1-7-0-3-3-1=-4-7-2-3-1-8-$ $9-1-7-1--1--4-2-1-2--1-8-$
$9-1-7-3-3-1=-4-2-2-3-1-8-$ U CONCENTRATION WATER UNITS Ppb 
LASL Uranium Hydrogeochemical and Stream Sediment Reconnaissance Data Listing

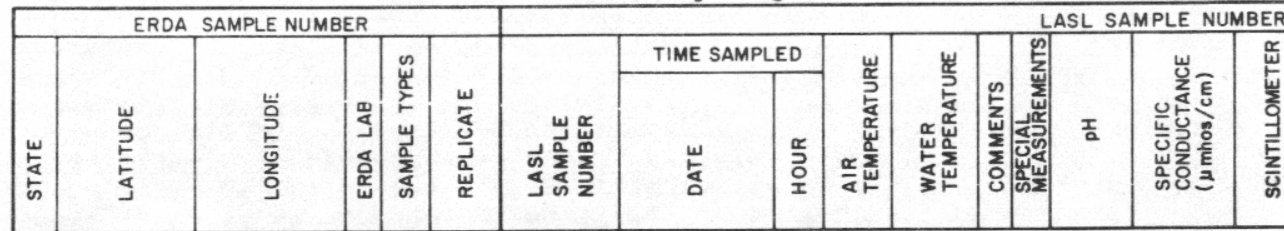

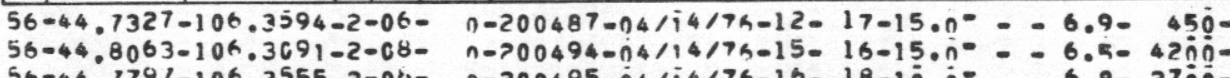

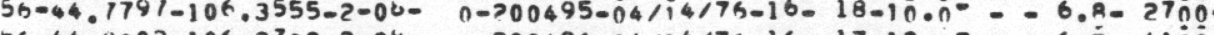
$36-44.8002-106.2702-2-08-0-200496-04 / 14 / 74-16-17-13 \cdot 0-0-0.5-4100-$ $56-44.9305-105 \cdot 7741-2-06-0-200501-04 / \hat{A} 7 / 76-11-19-17 \cdot 0^{\circ} C_{-}-6.9-15 n 0-$ $56=44.9650-105.8097-2-08-0-200504-04 / 77 / 74-12-12-8 \cdot n-C=-6 \cdot 7-2800-$

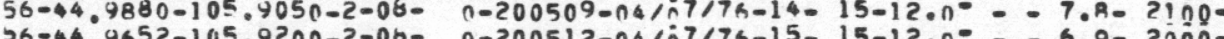
56-4. $9650-105.9413-2-06-0-200515-0404700$

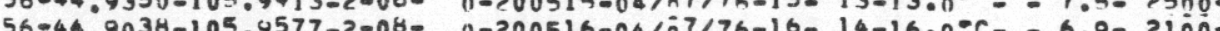
(56-44.9038-105. $56-44.9225-105.9050-2-08-n=200519-04 / n 7774-16-14-15 \cdot n-c-7.2-? n Q 0-$ 56-44.7769-105.8902-2-04- 0-200525-04/i8/7h-15-14-13.n-C- - 6.n- 2ño-

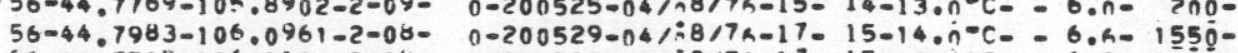

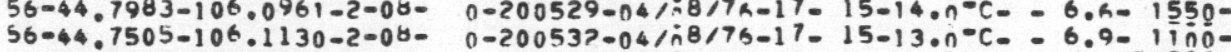

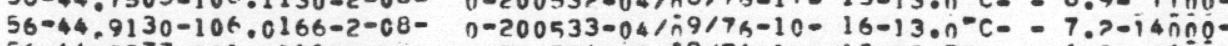
(1)

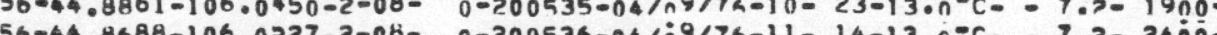
$56-44.8688-106.0027-2-06-0-200536-0472977 h-11-14-13.0-C=-7.2-2400-$ 56-4. $8461-10$ h. 0466-2-08- n-200538-04/i9/7h-11- 14-16.0-C- - T.2- 15ño$56-44.8380-106.0658-2-00-\quad n-200542-n 4 / i 9 / 7 h-13-24-14 \cdot n^{-C} \mathrm{C}=0.9-1600-$

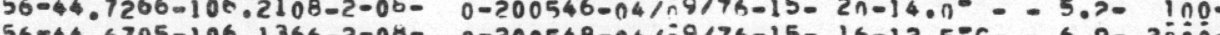
$56-44.6705-106.1366-2-08-\quad n-200548-04 / n 9776-15-16-12.5-C=-6.9-2 n n 0-$ $56-44.6988-106.2027-2-10-\quad 0-200551-04 / n 9 / 76-15-16-14 . n^{-}-0.9-15 n 0^{-}$ $36-44.4991-105.5491-2-06-\quad 0-200552-04 / 10 / 76-10-9-7.5^{\circ}-0-6.5-370_{0}-$ $56-44.4827-105.5625-2-00-n=200553-04110776-10-9-11 . n-c=-7.2-19 n 0-$ $56-44.4650-105 \cdot 5475-2-0 b-\quad 0-200554-04 / i 0 / 76-1 n-9-11 \cdot n^{-C} C_{-}-6.3-\quad 750-$ $56-44.4663-105.5694-2-08-0-200555-04 / 10 / 76-10-8-10 \cdot n^{-}=-6.5-8 n \overline{2} 0-$ $50-44.4111-105.5497-2-080$ n-2C0557-04/10776-11- 9-10.n- - - 6.5- $2900-$ $56-44.3866-105.5416-2-08-n-200558-04 / i 0 / 76-11-11-9 \cdot 0^{-0}-0.5-19 n_{0} 0_{-}$ 56-44.4302-105.5277-2-06- n-200559-04/i0/7n-13-1n-9.n- - - 6.6- 45056-44.4000-105.5175-2-06- 0-200560-04/10/7a-13-10-11.n- - - 7.2- 3ñก0$56-44.4530-105.6238-2-08-0-200561-04 / 10 / 75-13-14-12.0^{-}-0.5-4 n n Q-$ $56-44.4852-105.6838-2-08-0-700564-04 / 10 / 76-15-11-100 n=-0.60-33 n 0-$ 56-44.4736-105.7297-2-06- n-200565-04/10/76-15-13-1n -n - - 6.5- 45ño-

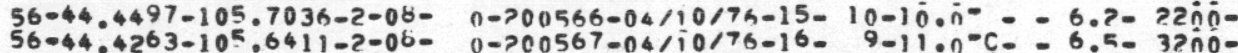
$56-44.4616-105.5827-2-06-0-200572-04 / 10 / 76-15-10-7.0=-6.902200-$ 56-44.3794-105.4961-2-06- 0-700573-04/12176-7- 8-7.0- - - 6.9- 6n0-

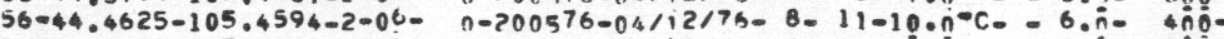

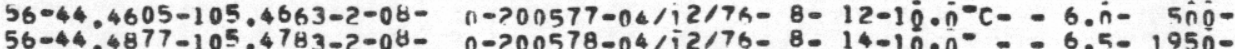

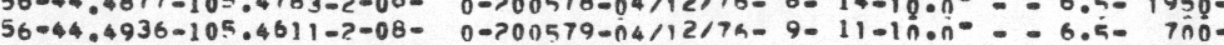
56-44.5183-105.4B61-2-06- n->00580-04/12/7a-9-14-12.10- - 6.h- 2ñno$56-44.5172-105.4519-? \cdot 08-n-200581-n 4 / 12 / 7 h-9-15-11 \cdot n^{-}-0.7-11 n 0_{-}$

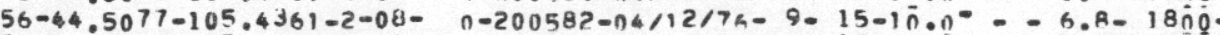
$56-44.4819-105.4000-2-06-0-200584-04 / 12 / 76-10-17-11 . n^{\circ}-0.9-700-$ $56-44.6102-105 \cdot 3997-2-06-0-200589-04 / 72 / 7 n-11-22-14 \cdot n^{\circ}=-6 \cdot n=4 n 0^{-}$ $56-44.5411-105.3508-2-08=0-200592-04 / 12 / 7 B-12-26-12.5-$ - - 6.9- $2900^{\circ}$

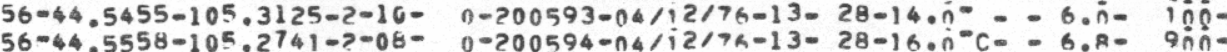

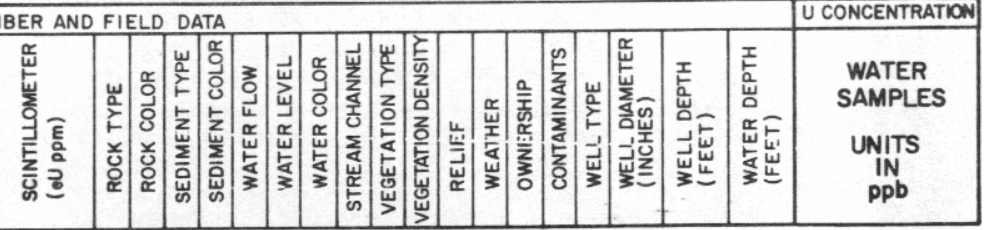

$10-1-7-5-6-1-2-2--4-2-2-3--10-$ $6-1-7---2-2-1--4-2-2-5--1-1=$ $7-1-7--2-2-1--4-2-2-2--1-1$

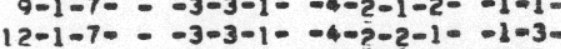

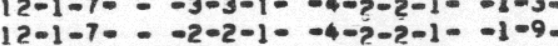
$12-1-7-=-2-2-1--4-2-2-3--1-1-$

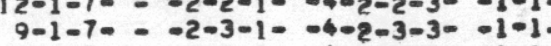
$12-1-70=-2-2-3--4-2-2-2=-1-1$ $13-1-7--2-3-1--4-2-3-2--1-9$ $15-1-7-=-3-3-1--4-2-2-2--1-1-$

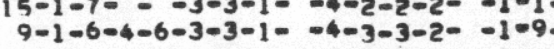
$12-1-6-4-6-1-2--4-3-2-2--10$ $9-1-7--3-3-1=-4-\bar{c}-3-1=-1-9-$ $12-1-70-7=3-3-1=-4-2-3-1=-1-9$ $12-1=6-4-6-1-2-1--4-3-2-1--1-9$ $6-1-6-4-6-2-3-1<-4-3-3-1-=1-1$ $15-1-6-4-6-3-3-1=-4-3=2-1--1$
$6-1-6-4-6-2-3-1=-4-3-2-1--1-9$ $6-1-6-4-6-2-3-1--4-3-2-1--1-9$
$12-1-6-4-6-3-3-1--4-3-2-1--1-8$ $12-1-6-4-6-3-3-1=-4-3-2-1--1-8-$
$9-1-6-4-6-3-3-10-4-3-1-1--1-9-$ $9-1-6-4-6-3-3-1=-4-3-1-1--1-9$
$9-1-6-4-6-1-2-3-0-4-3-2-2-1-1$ $9-1-6-4-6-1-2-3=-4-3-2-2=-1-1=$
$15-1-6-4-6-3-3-1-0-4-3-3-4-1-1-9=$ $9-1-6-4-6-1-2-4--4-3-3-4--10-$ $6-1-7---2-2-1--4-2-2-3--1-1-$

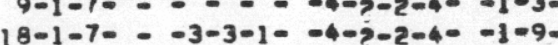
$18-1-7=-3-3-1=-4-2-2-4--1-9-$
$6-1-7-0-0-0-4-2-2-4--1-10$ $6-1-7-1-1-2-1=-4-2-2-4-1-1$
$18-1-70-1-2-3-1=-4-2-2-3-1-10$
$9-1-70-10$ $9-1-7--3-3-1=-4-2-2-3--1-1-$ $2-1-7-1-1-1-4-2-2-3-1-1$ $6-1-7-5-8-2-3-1--4-2-2-3--1-1$
$15-1-7-7--3-3-1--4-2-2-3--1-1$ $15-1-7-0-3-3-1=-4-2-2-3--1-1-$
$12-1-70=-2-3-1=-1-7-2-3--1-1-$ $12-1-7=-2-2-3-1=-1-7-2-3--1-1=$
$9-1-7-0-2-3-1=-1-7-2-3--1-1$ $6-1-7-=-2-3-1=-4-2-2-3--1-1-$
$15-1-7-0-2-3-1=-4-2-2-3--9-1=$ $10-1-7---2-2-1--4-2-1-3--1-1-$ $1>-1-7---2-2-1--4-2-2-3--1-1=$ $12-1-7-4-6-3-3-1=-4-2-2-3--1-$

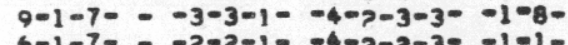
$6-1-7--2-2-1=-4-2-2-3--1-1-$
$7-1-7--2-3-1--4-2-3-3--1-3-8-200$ $15-1-7--2-3-1--4-2-3-5--1--0$ $2-1-7---3-3-1--4-2-2-2--1-1=$ $12-1-7--2-2-1--4-2-2-2--1-1=$ $15-1-7-4-6-2-2-1=-1-2-3-3--1=$ $9-1-7--1-2-2--4-2-1-1--1-1-$

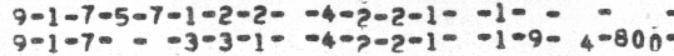


LASL Uranium Hydrogeochemical and Stream Sediment Reconnaissance Data Listing

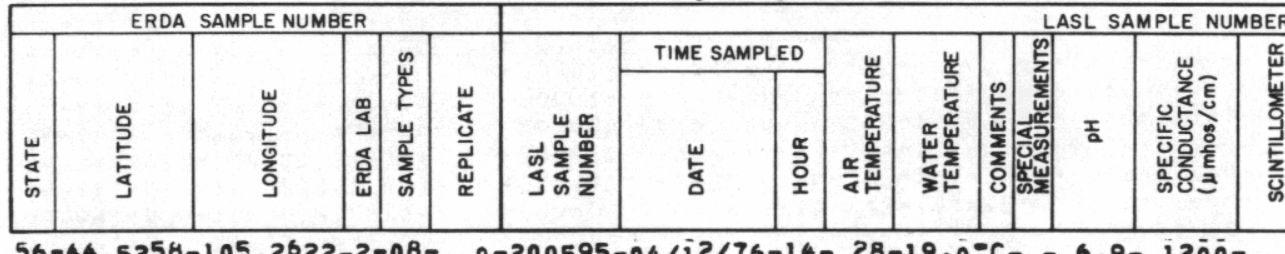

年 $36-44.5344-105.2747-2-08-0-200597-04 / i 2175-14-27-16.0-0-7.2-13 n \overline{0}-$ $56-44.5825-105.3127-2-08-0-700598-04 / 12 / 7 b-15-27-19 \cdot n=-0.8-1150-$ 56-44.5827-105.3366-2-08- 0-200599-04/12/75-15- $28-19.0-=-6.9-1200$

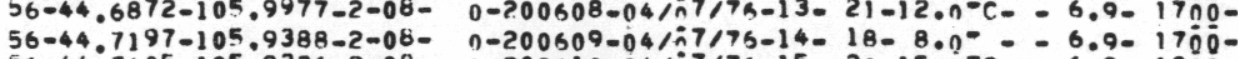
56-44.7436-105.9358-2-06- 0-200612-n4/i7/7h-16- 18-9.n- - G.R- $40 \hat{n} 0-$ $56-44.7122-105.8913-2-08-0-200614-04 / 78 / 7 h-16-15-9.0-0-6.3-5 n n 0-$

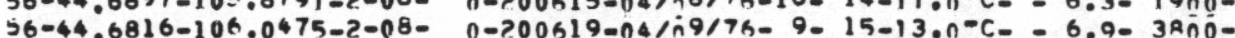

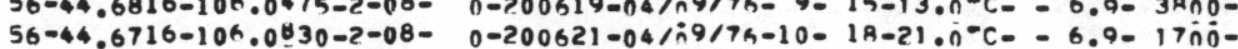

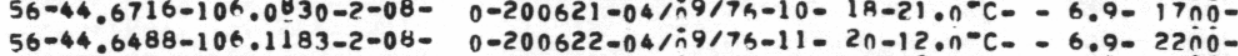

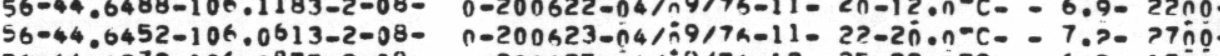
$56-44.6372-106.0875-2-08-0-200625-04 / j 9 / 76-12-25-22.0-c-0.9-17 n 0-$ $56-44.6355-106.0200-2-08-0-200626-04 / 19 / 76-12-25-22 \cdot n-C_{-}-6.9-2200-$ $56-44.6194-106 \cdot 025 n-2-08-\quad n-200627-04 / i 9 / 7 h-13-25-15 \cdot n-c-C=6.9-6 n d-$

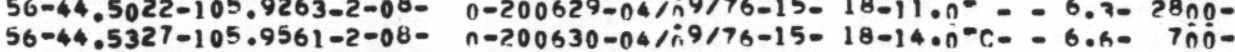
56-44.5383-105.9486-2-08- 0-20n632-04/:9/7h-16- 15-12.n-C- - 6.h- 6njo-

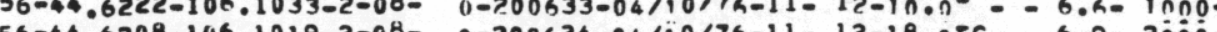
56-44.6208-106.1019-2-08- n->00634-04/10/76-11- 12-18.n-C- - 6.9- 2 nño$56-44.5211-106.1097-2-08-0-200636-04 / 10 / 76-12-12-18 \cdot 0_{-1}^{-C}-6.9-1890-$ (1) $56-44.6002-106.0927-2-08-n-200640-04 / i 0 / 7 n-13-12-10 . n-C-6.8-15 n 0_{-}^{-}$ 56-44.6980-106.1163-?-08- 0-200641-04/i0/7A-14- 11-17.n-C- - 6.9- $17000-$ 56-44.7305-106.1197-2-08- n-20064?-04/10/76-14- 14-14.n-C- - 6.h- 11 $56-44.7463-106.1166-2-06-0-200643-04 / 10 / 7 h-14-14-16 . n-c=-6.0-18 n n-$ $56-44.5494-106.1936-2-09-\quad 0-200649-04 / 1217 h-10=20-13 . n^{\circ}=-6.3-450$ 56-44.4933-106.1744-2-08- 0-200651-04/12/76-14-26-18.n-C- - 7.P- ?2ñn-

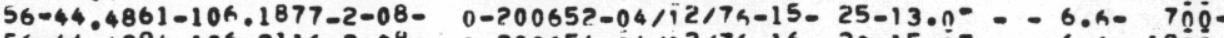
56-44.4894-106.2116-2-08- n-200654-n4/12/76-16- 2n-15.n- - 6.h- 1900 $56-44.4627-106.2994-2-08-0-200657-04 / i 3 / 76-9-22-13.5-C-C-6.9-8000-$ $56-44.4458-166.2497-2-08-0-700659-04 / 13 / 76-10-22-15 \cdot 0-C-0.909-31000-$ $56-44.4366-106.3494-2-08-0-200660-n 4 / 13 / 7 h-11-22-14 \cdot n-C=-6 . h-1100-$ $56-44.4216-106.3333-2-08-0-200662-n 4 / 13 / 7 h-13-22-17 . n-c=-6.9-13 n 0-$

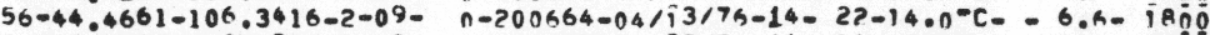
56-44.4844-106.1341-2-08- 0-700667-04/i3/7h-14-14-13.5-C - - 6.9-22ñó$56-44.4822-106.1422-2-08-0-200668-04 / 13 / 76-14-15-15 \cdot 0-\mathrm{C}-0.6 .8-1850-$

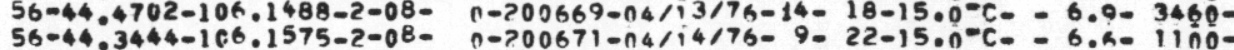
$56-44.3794-106.1600-2-08-0-200673-n 4 / 14 / 76-9-16-18.0-C=-6.9-550-50$ 55-44.3175-106.1458-2-08- 0-200677-04/14/7b-10-14-19.0- - - 7.ñ- 18 ñó-

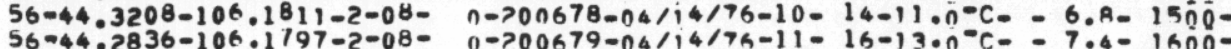
$56-44.4755-106.2783-2-08-$ $36-44.2911-106.2236-2-c 8-$ $56-44.2694-166.1458-2-08-$
$56-44.2822-106.1275-2-08-$ $56-44.2605-106.1708-2-08-$ $56-44.8502-106.2061-2-68$

$0 \rightarrow 200480-04 / 13 / 7 h-8-22-12 . n-C_{-}-6 . h-21 n i n-$ $0-200681-04 / 14 / 76-11-16-12 \cdot n^{-C}-7 . ?-3900-$ $n-200682-04 / 14 / 74-12-16-14.5-\mathrm{C}-7.1-1>n 0-$ $0-200684-04 / 14 / 75-12-16-14 \cdot n^{-C}-C_{-}-6.9-1100-$ $0-200685-04 / 14 / 76-12-16-12 \cdot n^{-C} \mathrm{C}-=6 . \mathrm{h}-5 \mathrm{n} 0-$ $0-200701-04 / 14 / 76-17-14-13.0^{-}-0.3-2 n^{\circ} 0^{-}$

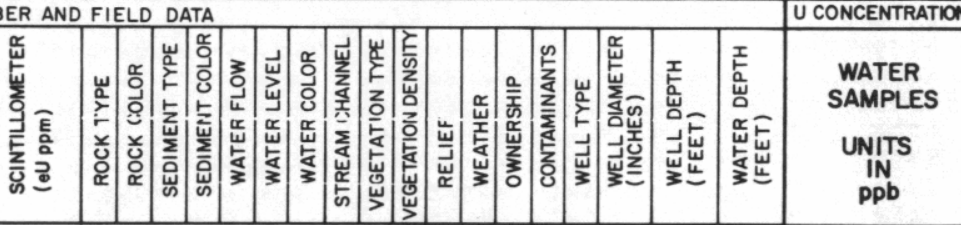

9-1-7-- -3-3-1- -4-2-2-1- -1-9$7-1-7-=-2-3-1--4-2-2-1--1-1=$

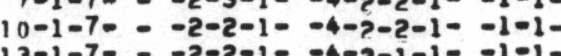

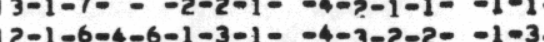
$-10-3-2-2--1=3$ $16-1-6-4-6-3-3-1=-4-3-2-4-4-3-3-6-350=$ $6-1-6=4-6=2-3-1=-3-2-4-2=-1=$ $6-1-6-4-6-2-3-1-4-3-2-4-2--10$ $12-1-6-4-6-2-3-1-4-3-2-1=-1-1$ $15-1-6-4-6-1-2-1=-4-3-2-1=-1-1=$ $15-1-7-4-7-3-3-1=-4-3-2-1=-1-9-9$ $9-1-7-4-7-3-3-1--4-3-2-1--3-9$
$9-1-6-4-6-2-3-1--4-3-2-1--1-9$ $9-1-6-4-6-2-3-1--4-3-2-1--1-9$
$12-1-6-4-6-2-3-1=-4-3-2-1=-3-9$ $12-1-6-4-6-2-3-1=-4-3-2-1=-3-9$
$12-1-7-4-7-3-3-1=-4-3-2-1=-1-9$ $9-1-6-4-6-2-3-1=-4-4-2-1--1-9$ $16-1-6-4-6-2-3-1=-4-3-2-1=-1-9-$ $19-1-7-4-6-3-3-1=-4-3-3-2-1-3-10$ $16-1-7-4-7-1-3-1=-4-3-2-2=-1-3-3$ $9-1-6-4-6-1-3-1--4-3-2-2--1-3-$ $9-1-6-4-6-3-3-1=-4-2-2-3-3-3-3-\alpha=140$ $12-1-6-4-6-1-3-1--4-2-2-3--3-3-6-15 n-$ $\begin{aligned} 12-1-6-4-6-2-3-1=-3-2-3-3- & -1=9-\end{aligned}$ $12-1-6-4-6-2-3-1=-4-3-2-3--3-9$ $12-1-6-4-6-2-3-1--4-3-2-3--1-9$ $13-1-6-4-6-2-3-1--3-2-3-3--1-9$ $12-1-6-4-6-3-3-1=-3-2-2-3--1-9-$ $9-1-6-4-6-2-3-1--2-2-2-1--1-9-2-800$ $9-1-6-4-6-2-3-1--2-2-3-1--1-3-6-13$ h$15-1-6-4-6-2-3-1=-2-2-2-2-=1-9$ $1-1-6-4-6-1-2-1--4-2-3-2--1-1-$ $4-1-6-4-6-2-3-1=-3-3-3-2=-1-9$ $9-1-6-4-6-2-3-1--3-3-3-2--1-1-$ $6-1-6-4-6-1-3-1--4-3-3-2--1-3$ $12-1-6-4-6-1-3-1--3-4-3-2--1--$ $12-1-6-4-6-2-3-1--4-3-3-4-=9-9$ $19-1-6-4-6-3-3-1=-4-2-3-3--1-9-$ $9-1-6-4-6-1-3-1--4-3-3-2-1-1-9-$
$16-1-6-4-6-2-3-1--3-2-3-2--1-4-$ $12-1-6,4-6-2-3-1=-4-3-3-3=-1-3$ $12-1-6-4-6-2-3-1--4-3-3-3--1-6-$
$12-1-6-4-6-2-3-1--2-2-3-2--1-3-$ $9-1-6-4-6-2-3-1=-4-3-3-2 \div-1-9$ $12-1-6-4-6-2-3-1--3-2-3-2--1-9$ $6-1-6-4-6-1-3-1--3-2-3-3--1-1-$ $16-1-6-4-6-1-2-2--3-2-2-2--1-1-$ $16-1-6-4-6-3-3-1=-4-3-2-2--1-9-$
$16-1-6-4-6-2-3-1--2-2-2-3--1-9$ $16-1-6-4-6-2-3-1--3-2-3-2--1-9$ $11-1-7---2-2-1--4-2-2-2--1-1-$ $\mathrm{ppb}$ 
LASL Uranium Hydrogeochemical and Stream Sediment Reconnaissance Data Listing

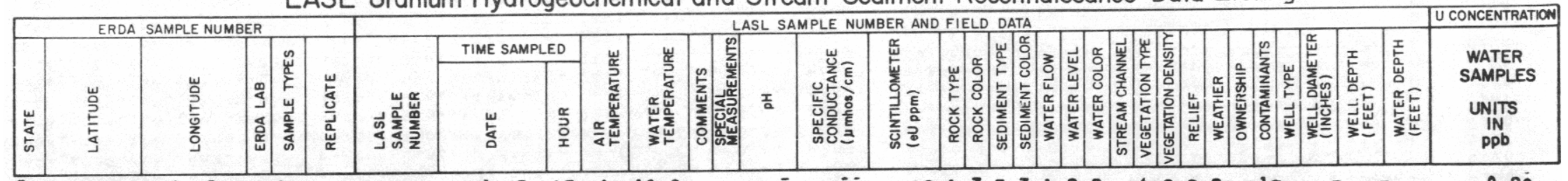

$56-44.8300-106.2233-2-10-0-200702-04 / 14 / 76-17-14-13.0--0 . \overline{6}-40 \overline{0}-$ $56-44.8141-106.2263-2-08-0-200704-04 / 14 / 76-18-13-14 \cdot 0-0-6.3-1100-$ $56-44.7666-164.1772-2-C B-0-200707-n 4 / 15 / 7 B-8-14-14 \cdot n-C-D-7 . n-10 n 0-$ $36-44.8961-10 \mathrm{~K} .2975-2-08-0-200716-04 / 15 / 76-11-18-12 \cdot 0^{-C} \mathrm{C}--6.8-2 ? 00-$

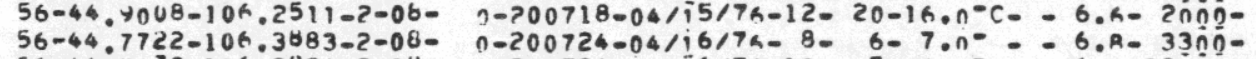

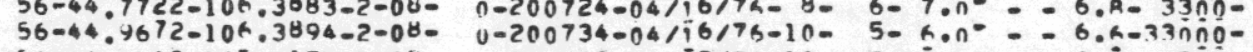
$56-44.2113-105.9172-2-08-n-200738-04 / 99 / 76-10-9-1 n_{0} 0-0-6 . n-3 n 0-$

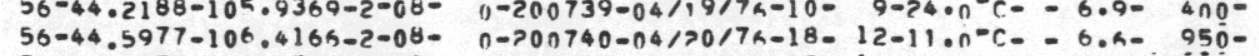
$56-44.6372-106.39 n n-2-08-n-200747-n 4 / 21 / 7 a-12-13-11 \cdot n-C-0.3-17 n n 0-$ $56-44.5644-106.4788-2-08-00-200756-04 / 21 / 76-14-14-13.0-0-8.1-1$ inñ$56-44.5422-106.4744-2-08-0-200758-04 / 21 / 74-14-14-14 \cdot 0^{-}:-6.3-37 n 0^{-}-$ $56-44.5852-106.4636-2-08-00700762-04 / 21 / 76-15-14-24.0-C_{-}-6.9-7 n 0^{-}$

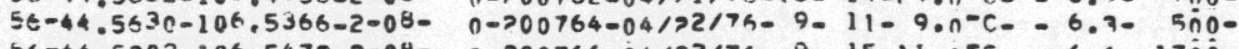

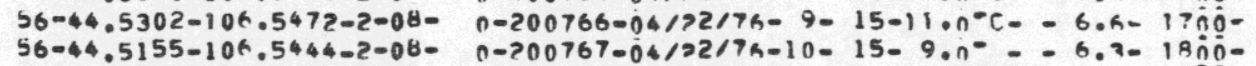

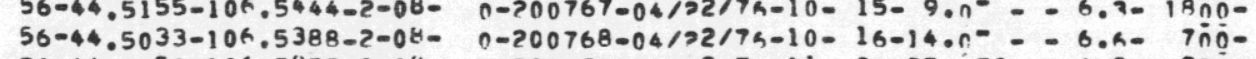
$56-44.5650-106.5855-2-08-0-200770-n 4 / 22 / 7 a-11-2 n-25 \cdot n-C-D-6.9-950-$

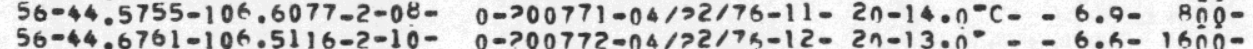

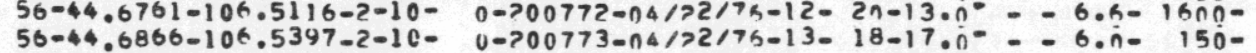
$56-44.7100-106.5344-2-06-00-700774-04 / 22 / 76-14-21-15.0-=-5.7-45-$ $56-44.7111-106.5658-2-06-0=20 n 775-04 / 22176-14-21-17 \cdot 0^{\circ}-0.6 \cdot n-1150-$ 56-44.0416-106.5383-2-06- n->00779-04/32/7h-15-19-16.n- - - 6.h- AnO$56-44.6491-106.5063-2-10-0-200780-04 / 22 / 7 h-15-21-13.00^{-}-0.6-$ - BnO$56-44.6502-106.5969-2-08-0-200783-04 / 32 / 7 h-15-20-120 n^{\circ} \mathrm{c}=-6.9-430-$ 56-44.6152-106.5891-2-08- 11-300784-04/32/7h-15-19-1h-n-C= - 6.1- 9ñ$56-44.5977-10 \% .5908-2-10-0-200785-n 4 / 32 / 7 a-16-18-14.0-0-6.9-12 n 0-$ $36-44.5900-106.5655-2-10-0-200786-04 / 72 / 76-16-20-14 . n-$ - - 6.5- $1350-$

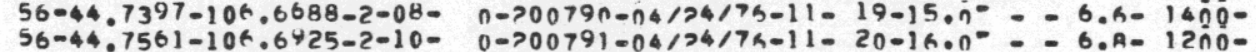

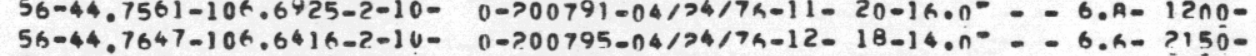
$56-44.7516-104.6033-2-0 b-n-200796-04 / 24 / 7 h-12-19-16 . n=-0.4-13 n 0-$

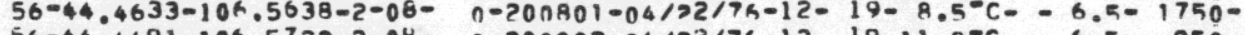
$56-44.4491-106.5722-2-08-0-200802-04 / 32 / 76-12-19-11.8-C=-6.5-950-$ 56-44.4386-106.5855-2-08- 0-700803-n4/22/7a-13-19-100n- - - 6.a- 12 ño$56-44.4250-106.5+38-2-08-n-2.00905-04 / 22 / 74-14-22-13 . n^{-C} C_{-}-6.7-1150-$

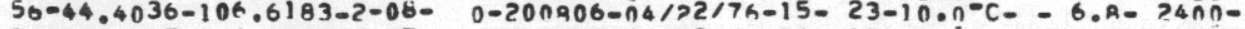
$56-44.5047-106.8038-2-07-n-200807-n 4 />2 / 7 B-16-17-14.0=-0.8-1250-$ $56-44.4913-106.8033-2-07-0-200808-04 / 32 / 7 h-17-16-12.5^{\circ}$ - - 6.A- 7ño56-44.3283-106.692?-2-06- n-200R09-04/33/76-9- 9-16.0-C- - 6.R- $2400-$

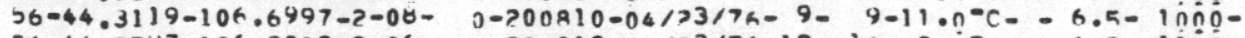

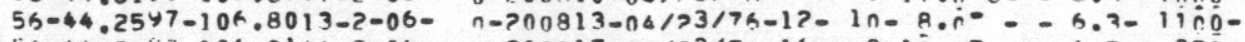
$56-44$.3083-106.8144-P-06- 1)-200317-04/33/7R-16- 8-100n- - - 6.5- 950-

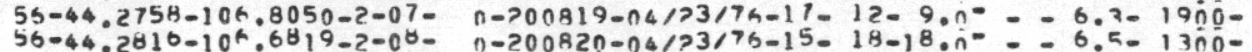
$56-44.3716-106.6408-2-08-n-200821-04 / 34 / 74-9-8-1 n \cdot 0^{\circ}-0.5-650-$

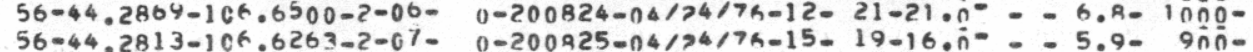

$15-1-7-5-7-1-2-2--4-2-2-2--1-1=$

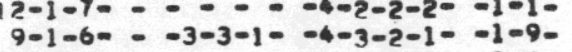
$6-1-6--3-3-1=-4-3-3-1--9-2-$ $3-1=6--2-3-1--4-3-3-2--1-9-$

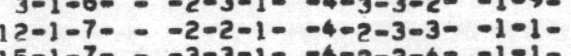
$9-1-60--1-2-1=-4-3-2-2--1-1-$

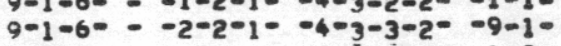
$0-1-7-\ldots-0-4-5-1-1--1-8-$ $12-1-6--3-3-1--4-3-2-2=-1-9-$ $6-1-6=-3-3-1--4-3-3-2--1-8-$ $12-1-6--2-2-1=-4-3-3-1--1-2-$ $9-1-6---2-5-1=-4-3-3-1=-1-5-$ $9-1-6--3-3-1--4-3-2-1--1-8-$ $9-1-7---3-3-1--4-2-1-2--1-9-$ $9-1-7---3-3-1--4-2-1-2--1-3-4-1000$ $9-1-7--3-3-1--4-2-5-5-1-1-1-$ $9-1-7-=-3-3-1--4-2-1-2--1-1-<10$

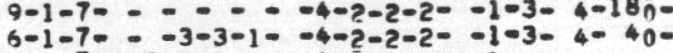

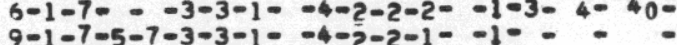
$0-1-7-5-7-1-2-2--4-7-2-1--10$
$9-1-7-5-7-3-3-10$ $9-1-7-5-7-2-2-1--4-2-3-5--1=-$ $9-1-7-5-7-2-2-1--4-2-3-2--10-$ $9-1-7-0-0---4-2-2-5-1-1-$ $12-1-7-5-7-2-2-2-4-4-2-2-2--10-$

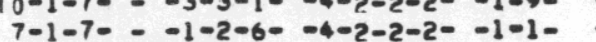
$7-1-7-5-1-1-2-6--4-2-2-2--1-1-$
$7-1-7-5-7-3-3-2--4-2-2-2--1-$ $12-1-7-5-7-2-2-2-0-4-2-2-2--1-$ $6-1-6--3-3-1--4-3-2-1--1-1=$ $12-1-6-4-6-1-3-1-1-6-3-2-1-1-1-$
$9-1-6-4-6-1-3-1-0-4-3-2-1--1-$ $6-1-6-4-6-2-2-1--4-3-3-1--1--\ldots$ $17-1-1=-\ldots-1-3-2-2-1-1-3-4-2000$

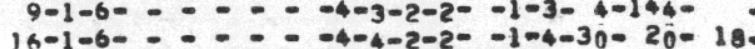
$12-1-6=\ldots-0-4-3-2-2--1-3-$ $11-1-6--1-1-1-4-4-3-2--1-9-$
$9-1-6-4-6-2-2-1-2-4-4-3-3--1--$ $9-1-6-4-6-2-2-1-2-4-4-3-3-3-1=$
$4-1-8-5-8-2-2-1--4-4-1-3--10$ $11-1-7-\ldots-0-4-3-2-2--1-3-$ $9-1-7-0---4-3-2-3--1-9$ $11-1-6-4-6-2-2-1--4-7-2-2--1=$
$14-1-6-5-6-2-2-1-2-4-4-4-2--1=$ $11-1-6-5-6-3-2-1--4-4-3-2--1-$ $3-1-6---2-4-3-1-2--1-3-$ $9-1-6-1-8-6-8-2-3-1=-4-4-2-1=-1-3-$
$9-1-8-1-1-$ $6-1-6-4-6-2-2-1--4-3-2-1--10=$
0.90

0.27

0.15

3.81
1.57
1.84

1.86

0.2

0.10

0.10

4.4

0.10

. 27

4.96

0.3

0.15

0.10

4.9

0.10

0.10

.

1.26

..03

0.18

0.20
3.57

3.57

5.47

0.49

0.10

8.01

.

0.10

0.01

0.33

4.45

0.42

0.88

5.14

2.27

2.63

5.45

2.49

3.69

0.80

5.39

6.13

4.89

1.31

0.78

0.10 
LASL Uranium Hydrogeochemical and Stream Sediment Reconnaissance Data Listing

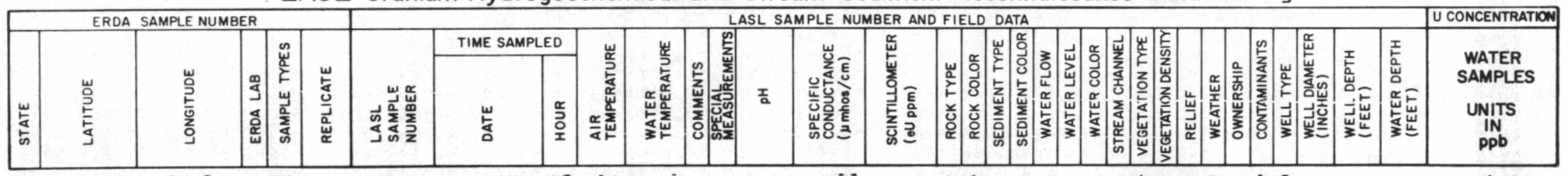

$56-44.3536-106.6730-2-08-n-200827-04 / 24 / 7 a-17-18-11.0^{-}=-6.5-9000-$ $36-44.7119-106.9716-2-08-0-200 B 28-04 / 26 / 7 A-9-5-16.0-1=-6.5-750-$ $56-44.8063-106.9030-2-06-0-200829-04 />4 / 76-11-18-18.0=-6.8-750-$

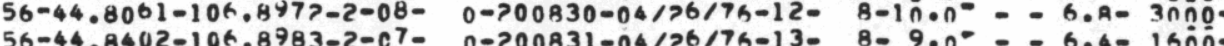
$56-44.8402-106.8983-2-c 7-0-200831-04 / 26 / 76-13-8-9.00=-6.4-16 n 0-$ $56-44.8472-106.8936-2-06-0-200833-04 / 26 / 76-13-16-8.00--6.7-950-$

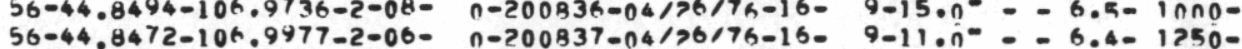

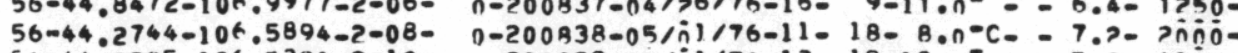

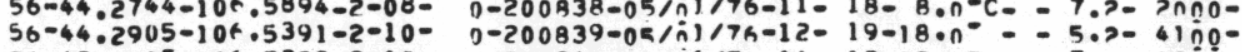

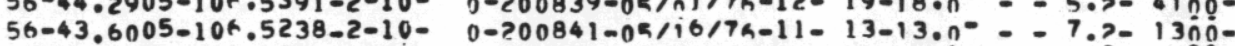

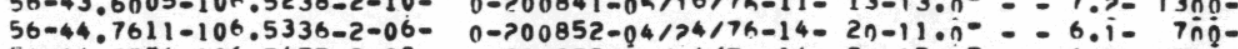

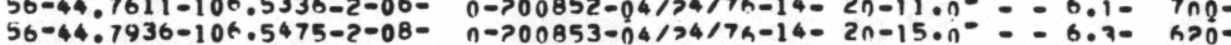
56-44.8655-106.5783-2-10- 0-200858-04/24/76-15-18-16.0- - 6.7- iñó

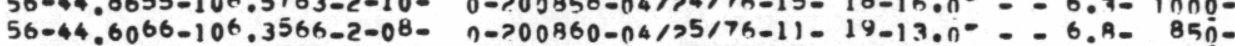
$56-44.60150-106.2777-2-10-00-200864-04 / 25 / 79-12-20-16.0^{-}=-6.30$ - $270-$

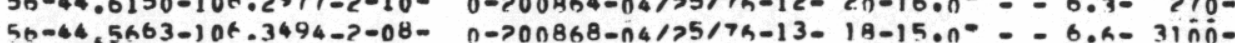

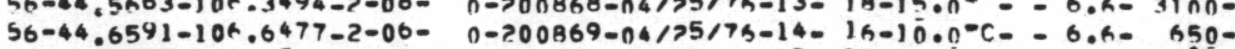

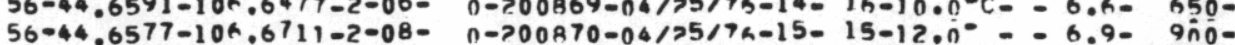

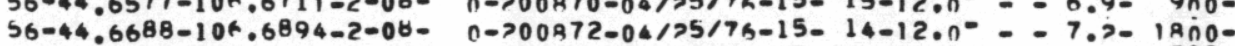

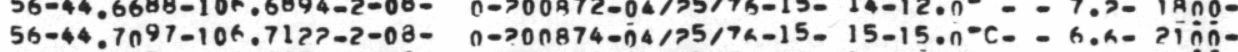
$30-44.6550-106.7450-2-08-n-200277-04 / 25 / 76-13-16-10.0=-0.2-1100-$ 50-44.6401-10k.718.

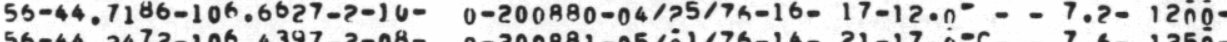

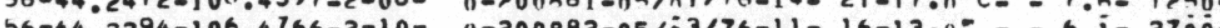
$36-44.2294-106.4766-2-10-0-200882-05 / 23 / 7 h-11-16-1300=-6.1-27 n 0-$

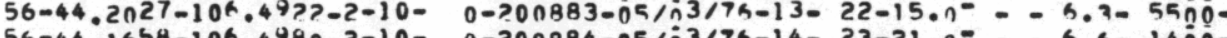

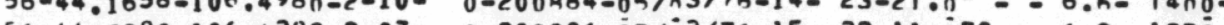
$56-44.2880-106.428 R-2-07-0-200886-05 / 23 / 7 A-15-22-11 \cdot \hat{n}=C-C=0.9-1250-$

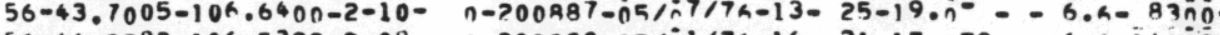
$56-44.3283-106.5322-2-08-0-200888-05 / 1+1 / 76-16-21-17 \cdot n^{-C} C=-6 . h-140 n 00-$

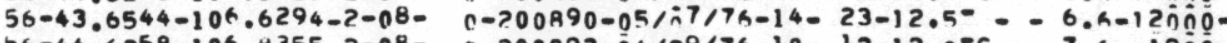

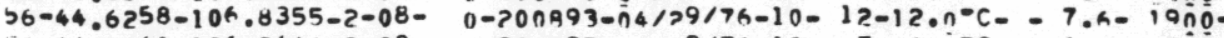
$56-44.6441-106.8444-2-08-\quad n-200895-04 / 29 / 7 h-10-7-6.0^{-C} C-$ - 6.h- Bño-

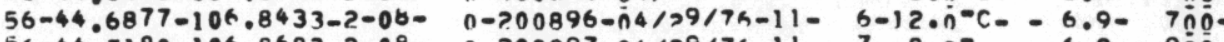
$56-44.7180-106.8683-2-08-0-200897-04739 / 75-11-7-8.0^{\circ}-0.6 .9-90^{-}-0$ 56-44.7763-106.8275-2-08- 0-700R99-n4/>9/76-12- B- 8.n-C- - 7.4- 2ñó$56-44.2455-105.6916-2-08-0-200900-04 / 19 / 76-8-1 n-9.0^{\circ} C-C-6.9-32 n 0-$ 56-44.7594-106.8338-2-08- 0-200001-04/79/76-13- 8-11.5-C- - 5.R- BO$56-44.7600-106.8422-2-08-0-200902-n 4 / 29 / 75-13-9-11.0-C=-6 . A-\operatorname{Bn} n \overline{0}-$

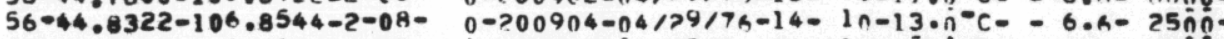
$56-44.8058-106.7625-2-08-\quad n-200905-04 / 29 / 75-14-1 n-100.0-C-6.9-700-$ 56-44.7402-106.7519-2-08- 0-200907-04/29/7a-15-11-16.0-C_ - 7.7- 12 no$56-44.7222-106.8025-2-10-00-200910-04 / 39 / 7 h-15-10-7.0-=6.9-$ h5056-44.7000-106.8666-2-08- n-20091?-n4/39/7h-16-12-17.n-C- = h.h- Rñ56-44.8568-106.8744-2-08- n->00913-04/30/7h-9-1>-9.n- - - 6.3- 9no56-44.9547-106.8500-2-64-0-700916-04/30/7h-11-17-11.00- - 6.0- $2800-$ 56-44.9716-106.7772-2-08- n->00917-04/78/7h-11-17-14.n-C- - 7.P- P2no-

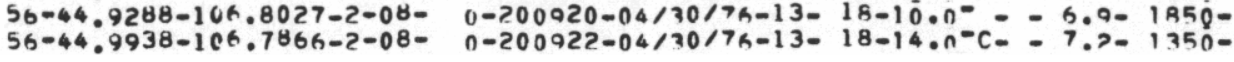
56-44.9127-106.86n5-2-08- n->n09915-04/90/76-10-2n-21.n- - - 6.9- 1ñño- $6-1-6-\ldots-0-4-3-2-1--1 \cdot 3-$ $12-1-6-\ldots-0-4-3-1-3--1-4-$ $6-1-6-1-0-3-3-1-3--1-4$ $3-1-8-6-8-2-2-1=-4-3-2-3--1-$

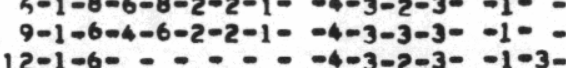
$11-1-6-4-6-2-2-1--4-3-2-3--1-$

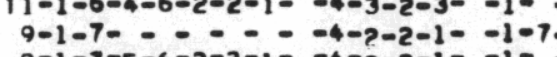

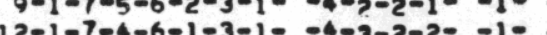
$15-1=6-4-6-1-3-1=-4-3-2-2=-10$ $6-1-6-4-6-2-3-1=-4-3-3-1=-1=$
$9-1-6--2-2-2-1=-4-3-3-1=-1-2=$ $9-1-6-4-6-1-3-1--4-3-3-1--1-$ $9-1-7---3-3-1=-4-2-2-2--1-1$ $9-1-7-5-3-3-1=-4-2-2-2=-1-1=$
$15-1-7-5-7-2-2-2=-4-2-2-2=-1-$

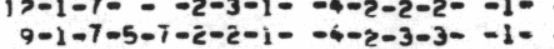

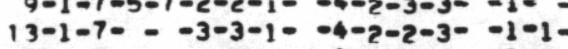
$21-1-7--3-3-1--1-7-2-3--1-1-$

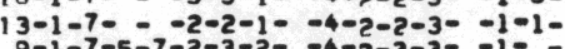
$13-1-7-5-7-2-2-2--4-2-3-3--10$ $14-1-7-0--1--4-7-1-1--1-1$ $12-1-7-5-7=---4-2-1-2--1=$

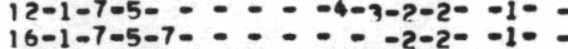
$11-1-7-10-1-10-4-3-2-3--1=$ $11-1-7=5-8-1-2-1-3-4-3-2-1=-10$ $9-1-7-0-0-4-3-2-1--1-1-$ 16-1-7- $13-1-4-3-1-1--1-8$ $13-1-7-=-3-3-1--4-2-2-3--1-8$ $13-1-7---2-2-1--4-2-2-3--1-8-$ 15-1-7- - -3-3-1- -4-2-2-3- -1-8$7-1-7-0-2-2-3-3--1-8-36-20=$ $9-1-6-4-6-3-3-1--4-2-2-1--9-3-$
$9-1-7-7--1-2-2--4-2-3-3--1-8-$ $20-1-7-=-1-1-2--4-2-3-3--1-6$ $12-1-7--\cdots--4-2-2-3--1-8$ $15-1-7-=-3-3-1--4-2-2-2--1-8-$ $12-1-7--3-3-1--4-2-2-3--1-3-4-19 n=$ 13-1-7-- - - - -4-2-3-3- -1-8$15-1-6--2-3-3-1=-4-3-5-1=-1-3-$ 6-1-6- - -3-3-1- -4-3-2-1- -1-8 $12-1-6=-3-3-1--4-3-3-1=-1-3-$ 6-1-6- - -3-3-1- -4-3-3-2- -1-9. $9-1-6-=-2-2-1--4-3-3-2--1-1-$
$6-1-6-=-3-3-1--4-3-3-2--1-9-$

$-180^{-}$

$-4 n=$

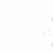
$1>-1-7---2-3-1=-4-2-2-5--1-$ $18-1-7--3-3-1--4-7-2-3--1-3-4-138$ $19-1-7-\ldots-0-4-2-3-2--1-8$ 6-1-7-5-7-3-3-2- -4-2-3-3--1--

$-160-$ $-200-$

$-\quad$

-

$\div$

$-$

$-$

$-$

$\mathrm{ppb}$

4.92

0.10

0.10

5.35

8.28

1.32

0.86

0.86

0.27

4.05
3.72

5.31

5

0.91

4.16

2.86

1.23
3.99

0.11

7.04

7.54

0.10

0.44

2.43
0.18

0.10

0.86

5.73

5.85

8.42

2.94

2.67

7.29
0.88

1.18

0.36

7.23

1.70

0.10

3.64

1.74

2.01

0.74

4.88

0.38

0.10 
LASL Uranium Hydrogeochemical and Stream Sediment Reconnaissance Data Listing

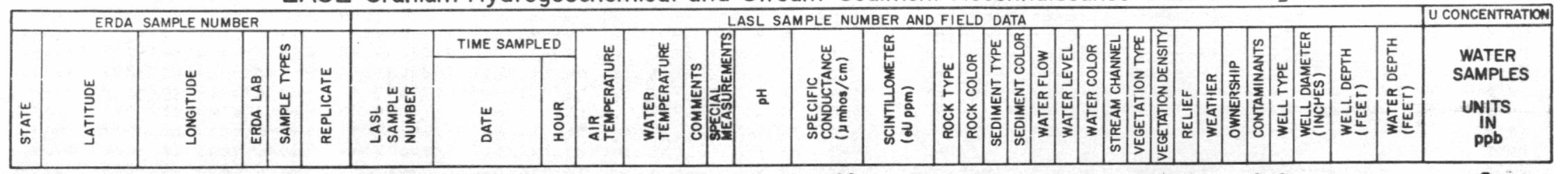

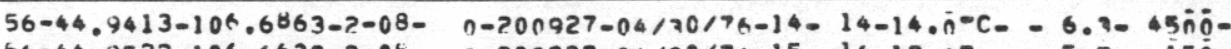
$56=44.9522-106.6633-2-06-$ $56=44.9741-107.0966-2-10$ $56-43.5525-106.5530-2-68-$ $56-44.0277-104.6272-2-08-$ $36-44.0855-106.6722-2-07-$ $36-44.0855-106.6722-2-07$ $56-44.0941-106.71116-2-00-$ $16-44.1672-10 \% .7422-2-10-$ $56-44.1847-106.7138-2-10-$ $56-44.2305-106.7105-2-08$ $56-44.0516-106.6950-2-07$ (36-40.2402-105.5480-2-09$36-44.1261-106.7236-2-10=$ - $44.1027-1$ 1 $540 n-2=10-$ $56-44.0750-106.6216-2-68-$ $36-44.0613-106.5816-2-07$ $36=44 \cdot 1055-106 \cdot 5697-2-10-$ $56-44.0444-106.4144-2-10-$ $36-43.5177-106.5177-2-10-$ $56-43.5200-106.523 n-2-08$ 56-4.5305-106. $5377-2-10$ $56-43.7261-106.6033-2-08=$ $26-43.5505-106 \cdot 1877-2-10=$ $56-43.5205-106.1372-2-08=$ $56-43.5205-106.1372-2-08-$ $56-44.8208-106.9475-2-08-$
$56-44.7750-106.9100-2=08-$ $56-44.7736-106.9455-2-08=$ $56-44.6755-106.9927-2-080$ $56-44.6433-106.9672-2-09=$ $56-44.6758-10+.9038-2-08-$ $56-44.6788-106.9223-2-07$ $56-44.6755-106 \cdot 9177-2-06$ $56-44.6800-106,9466-2-10$ $56=44.7061-106.9450-2-06$ $56-44.6000-106.8672-2-06$ $56-44.5750-106.8813-2-08$ 5o $-44.5747-106.9069-2-08$ $56-64.5700-106.8911-2-08$ $56=44.5788-106.9022-2-07$ $56-43.5866-106.6844-2-06-$
$56=43.6922-106.5313-2-06-$ $50-43.6788-106.5261-2-06-$ $56-43.6466-106.6044-2-10-$ $5 b=43.6977-106.4083-2-06$ $56-43.7322-106 \cdot 3122-2-16$ $56-43.7100-106.292 ?-2-06-$ $56-43.6827=106.290 n-?-10-$ $56-43.6858-106.3069-2-06$ $56-43.6950-106.2 \div 38-2-08-$ $n-200932-04 / 20 / 7 A-15-16-12.00=-5.7-150-$
$0-200943-05 / 11 / 7 a-11-13-12.0^{\circ}=-6.3-130-$

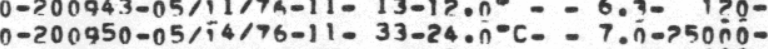

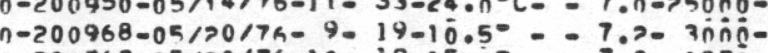
$0-200969-05 / 30 / 76-10=19-15.0^{\circ}=-7.8=1350$ $n->00971-05 / 18 / 74-11=2 n-12.0-0-7.4-390-$ $0-200973-05 / 18 / 74-12-27-14 \cdot 00^{\circ} \mathrm{C}-\mathrm{T}_{-7.4-34} \mathrm{C} 0 \mathrm{O}-$

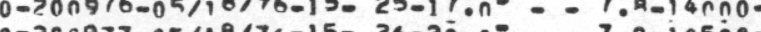
$0-200977-05 / 1877 a-15-26-20.0-2.8-105 n 0-$ n-200979-0a/i8/7a-16-26-20. $0^{\circ} \mathrm{C}=-\mathrm{T} \cdot \mathrm{h}-950-$ $0=200980-05 / i 8 / 76-18-25-14.00-2.20140-$

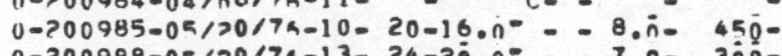

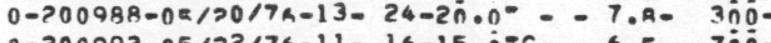
$0-200993-05 / 22 / 76-11-16-15 \cdot \hat{n}^{-C}-C_{-}-6.5-7 n^{\circ}-$

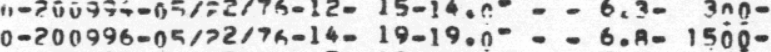
$000998-05 / 72 / 76-15-13-11 . n^{-1}=$ - 7.20 150 $0-201004-05 / 14 / 76-17-28-21 \cdot 00^{\circ}-7.9-300-$ - $201005-05 / 14 / 74-17-28-17 \cdot n-2$ - $7.8-28 n 0-$ $n-201006-05 / 14776-17-27-19.00^{-}-8 . n-14 n O-$ $n-201017-05 / 16 / 7 n-17-2 n-18 \cdot n-0-7 . A-950-$ $0-201018-05 / 17 / 76-13-26-18 \cdot n^{-C}-C_{-}-7 . ?-6 n i 00-$ $0-201021-05 / i 7 / 7 n-140-25-16 \cdot n-C=-7.8-9 n 0^{-}$ $n-201027-04 / 30 / 7 n-11-12-11,00=-6.8-750-$
$n-201028-04 / 30 / 74-12-10-11.5=-6.8-28100-$

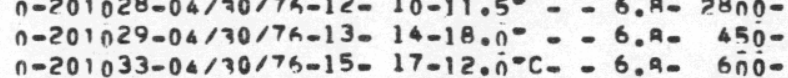
$n-201033-04 / 30 / 75-15-17-12 \cdot n^{-C} \mathrm{C}-6.9-6 n 0-$
$n-201034-04 / 30 / 7 n-16-14-12 . n^{-}-0.3-5 n 0^{-}$ $n=201035-04 / 30 / 7 n-16-16-13 . n^{\circ}-0.8-11 n 0-$ n-201038-0E/ñ1/73-12- 14-12.0 - - 6.R- 950-

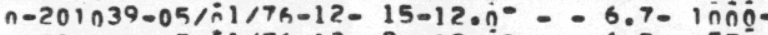
$0 \rightarrow>01040-05 / i 1 / 76-13-2 n-12 . n-2-6.5-550-$ $n-201042-05 / n 1774-14-18-12.0^{\circ}-0.7-950-$ $n \rightarrow>01043-05 / n 1776-15-18-15 . n^{-}-6.5-450-$ $n-201044-05 / i 1 / 76-16-17-11 . n^{-}-6.5-95$ $n-201045-05 / n 1 / 7 a-16-14-100 n^{-}=-6.5-1 n j-$ h->01046-05/i1/7h-16- 14-10.0 - - 6.50 1nQ $n-201048-05 / 51776-17-14-10.0^{-}-6.5-100-$

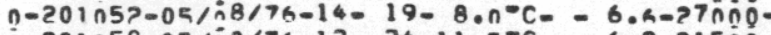
$\rightarrow 01058-05 / 10 / 76-12-24-11.5-C_{-}-6.9-315 n 0_{-}-$ $0-201059-05 / i 0 / 76-12-24-14.5^{\circ} \mathrm{C}-6.9-17 \operatorname{1n} 00-$ $0-201062-05 / 10776-14-24-18.55^{-}-6.5-11250-$ $0->01064-05 / 11 / 7 h-12-20-17 \cdot n^{\circ} C_{-}-6.5-14250-$ n-201066-05/11/76-17- 18-15.0- - 6.4-75nno$n-201067-05 / 11 / 76-18-14-1 n .5-\mathrm{C}=-6.4-1650-$ $n=201071-05 / 12 / 76-12-14-15 \cdot n-\mathrm{C}=-6.4-3850-$ n->01074-0a $12-1-6---2-2-1--4-3-3-1--1-3-$ $9-1-6--3-3-1--4-3-3-1--1-3-$ $15-1-7-4-6-1-2-3--4-3-2-4--1-0$
$15-1-70--3-3-1--4-2-2-1--1-1=$ $3-1-7--2-3-1--4-3-2-2--1-2-$

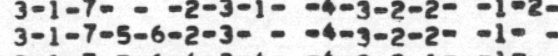
$12-1=7-5-6-4-3-4--4-3-3-1--1=$ $9-1-7--3-3-1--4-3-3-1=-1-9-$ $9-1-7-5-6-1-3-2--4-3-1-1=-1-$
$1-1-7-4-7-1-3-2--4-3-1-1--10$ $7-1-7-0-2-2-1--4-3-3-2--1-9-$ $7-1-7-7-4-2-2-1--4-3-3-2--1-9-$
$9-1-7-4-6-4-3-1--4-3-2-2--1=-$ $6-1-7-5-6-1-3-3--4-7-3-2--1=-$ $6-1-7-5-6-1-3-3--4-3-3-2--1=$
$6-7-6-5-4-1-3-3--4-3-3-2--1=$ $6-1-7-6-5-4-1-3-4-3-3-2--1-1-8-$ $6-1-7-7-6-4-4-4-2-4-2-2-3--1=8-$
$9-1-6-4-6-4-4-4-2-3-2-3--10-$ $7-1-6-5-8--4-2--4-3-2-3--10-$ $12-1-7-5-7-2-2-20-4-2-4-1--10=$

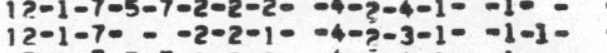

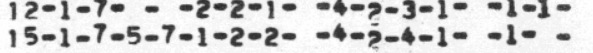
$15-1-7-5-7-1-2-2-1-4-2-4-1--1-$
$9-1-7---3-3-1--4-3-3-1--1-2=$ $9-1-7-5-7-2-3-1--4-2-2-1=-9-=$ $15-1-7-0-2--4-2--1=-1-8=$ $8-1-6=0=-4-3-2-2--1-4-$

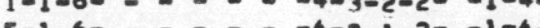
$5-1-6-\ldots-0-3-1-2--1-4-$ $8-1-6-1-6-4-6-2-2-1 \div-4-3-2-2--1-2-2-1-$
$2-1-6-4-2-$ b-1-6- - - - - - -4-3-2-3- - - $1-4=$ $16-1-6-4-6-2-2-1=-4-3-2-1=-1-=$ $19-1-6--1--1-4-3-2-1=-1-4-$
$3-1-6-3-6-3-2-1=-4-3-2-1=-10-$ $3-1-6-3-6-3-2-1=-4-3-2-1=-10$
$12-1-6-0-1-0-3-2-1=-1-4-$

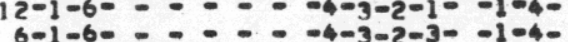
$9-1-4-\ldots-0-4-3-5-1--1-4-$ $9-1-40-0-04-3-5-1--1-4-$ $12-1-4--6-3-4-1--4-3-5-1--1-1-4=$ $9-1-1=-2-3-1=-4-3-3-1--1=$

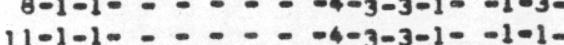
$11-1-1-1-1-1-3-4-3-3-1--1-1-$ $11-1-1-5-1=-3-1-3-4-3-2-3--1-2-240$ $14-1-1-5-7-2-3-1-2-4-3-2-2--1--$ $7-1-1--2-2-1-1-4-3-1-2--1-9-3$
$4-1-4-5-1--3-1-3-4-2-3-2--1-0$ $14-1-4-5-1--3-1-3-4-2-3-2--10$
$16-1-1--3--1--4-3-2-2--1-8-$ $16-1-1--3--1--4-3-2-2--1-8-1-8 j=$ 
LASL Uranium Hydrogeochemical and Stream Sediment Reconnaissance Data Listing

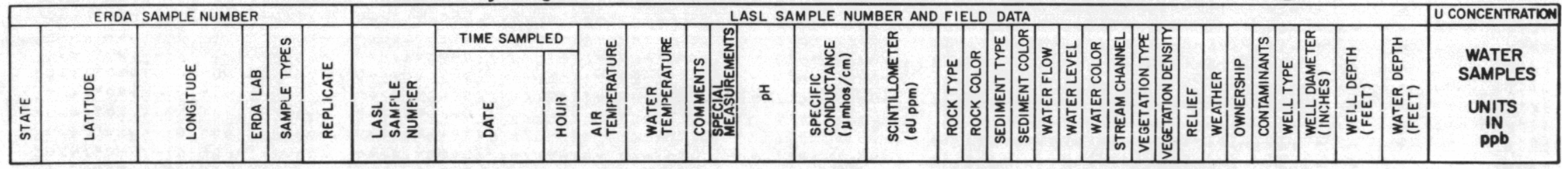

$56-43.8025-106.4338-2-68-0-201082-05 / 13 / 7 h-18-23-11.5-C$ - $-6 . h-22 n \overline{0}-$ $36-43.8055-106.4738-2-10-0-201084-05 / 14176-11-24-17.5-0-6.5-210-$ (56-430-

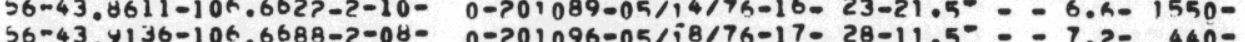
$36-43.7136-106.6688-2-08-0=201096-05178776-17-28-11.5=-7.7-440-$

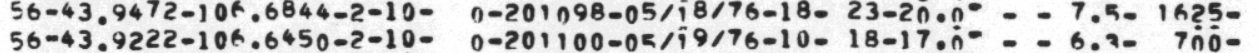

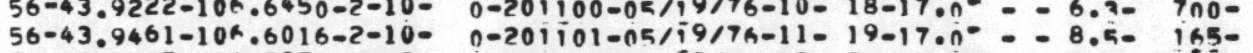

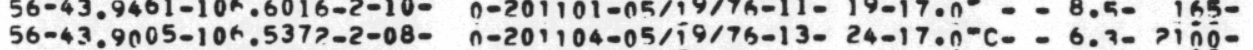

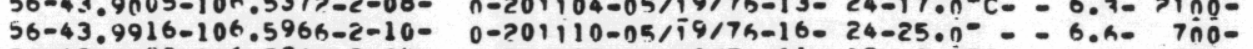
$56-43.8688-106.5261-2-06-00-701112-05 / 20 / 76-11-19-9.0-C_{-}-6.3-1750-$

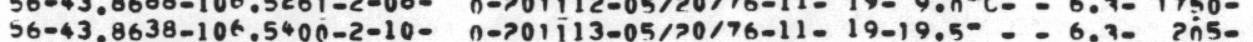
$56-43.8833-106.5133-2-08-00-201114-05 / 30 / 76-12-21-12.0^{-}=-6.3-1550-$

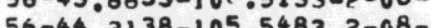
$56-44.2138-105.5433-2-08-$ $56-44.9550-1006.937-2-08-$ $56-44.9560-106.9605-5-07-$ $56-44.9560-106.9605-2-07-$ $56-44.9522-106.9416-2-08$. $56-43.5844-106.5097-2-10-$ $56-43.5844-106.5097-2-10-$
$56-43.5844-106.5061-2-10-$ $56-43.5400-106.4661-2-10-$ $56-43.5122-106.3969-2-08-$

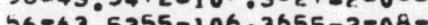
$56043.5355-10603655-5-08-$ $56-44.6977-104.9838-2-08-$ $36-43.5258-10<.6422-2-08=$ $56-43.6044-10 \% .6655-2=08=$ $36-44.4813-10$ h. $6488-2-08$ $56-44.2077-106 \cdot 3436-2-16-$ $56-44 \cdot 1750-106.2755-2-10-$ $55-44.2005-106.2738-2-10=$ $56-44.3702-106.327 ?-2-08=$ $3 B=44.4133-106.4483-2-08-$ $56-44.4150-106.3994-2-1 C-$ $56-44.4269-106.3755-2-10-$ $50-44$.3963-106.4416-2-08$56-44.1061-105.3186-2-06-$ $56-44.1322-105.405 ?-2-08$ $56-44.1652-105 \cdot 4100-2-08-$ $56-44.1905-105.3805-2-08=$ $56-44.2069-105.4108-2-08=$ $56-44.1936-105.4363-2-08=$ $56-44.1413-105.4544-2-04-$ $50-44.1677-105.4572-2-04-$ $36-44 \cdot 1902-165.4747-2-08-$ $56-44.2347-105.4207-2-08-$ $56-44.8211-107.0261-2-07-$ $56-44.8577-107 \cdot 0^{766-2-07-}$ $56-44.8283-107.0627-2-10-$
$56-44.7977-107.0097-2-10-$ $n=201125-04 / n 6 / 76-12-13-9 . n^{-C-C}-7.4-31000-$

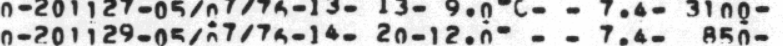

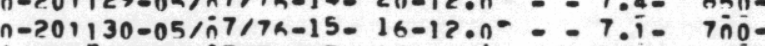

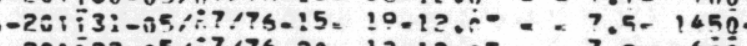
作 - $001136-n=/ 14 / 7 n-11-28-18 \cdot n-C=-7 \cdot A-23 n 0-$

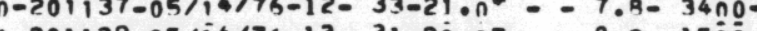
$0-201139-05 / 14 / 73-13-31-2000-0-8 . ?-15 n Q-$ n作 $n=201151-05 / n 3 / 7 h-12-18-15 \cdot n^{-}-0.5-5 n^{-}-6-$ n->01

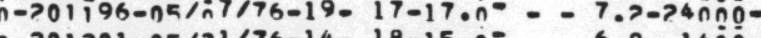
$0-201201-05 / 31796-14-18-15 . n-0.8-14 n 0-$ $0-201>11-05 / 1175-12-26-22.0^{\circ}-0.3-250-$

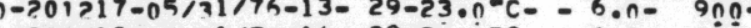
n-201>19-05/71/7n-14-29-200n-C- - 6.3- 6nOn-201224-05/z1/7h-17- 24-17. - $201327-05 / h 172-13-24-13 . n-C=-6.4=1550-$

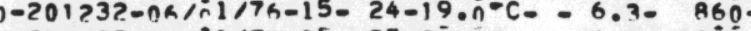

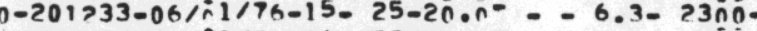

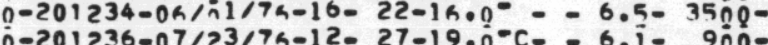

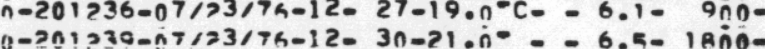
$0-201>340-n>1 / 33 / 7 a-13-30-1 k_{0} n-0-6.2-2800-$ $n \rightarrow 01241-07 / 23 / 7 n-13-30-16 \cdot n^{\circ}-0.6 .4-31 n 0-$ $0-201242-07 / 33 / 76-13-3 n-15.00=-6 . n-1900-$ $n-201>43-n>133 / 74-140-3 n-16 \cdot n=-6.2-24 n 0-$ $n-201>45-07 / 23 / 75-14-30-20 . n^{-}-C_{-}-6.7-7 n 0^{-}$ $0-201>44-n 7 / 23 / 76-14-28-24.00^{-}-6.5-27 n 0-$ $n \rightarrow 01>48-n 7 />3 / 76-14-26-15 \cdot n-0-6.3-3 n n 0-$

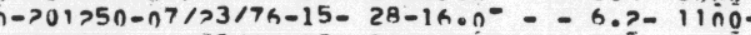
$n-201301-05 / 13 / 7 h-12-26-11 \cdot n^{-}-7.1-350-$ $n=201302-n 5 / 13 / 7 n-13-26-12 . n-0.8-300-$

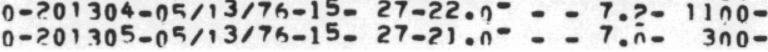

9-1-6- - - - - -4-3-1-1- -1-8$11-1-6-5-7-1-3-1-3-4-3-2-1--1--$ $10-1-1-5-7--2-1--4-3-2-1--1-=$

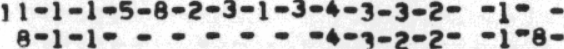
$15-1-1--1-3-1--4-3-2-2--1=$ $15-1-1-5-7-1-3-1--4-3-2-2--1=$
$11-1-7-5-7-1-3-3--4-3-2-1--10$ $11-1-7-5-1-1-3-3-4-3-2-1=-1-0$ $13-1-7-0-0-1--4-3-1-1--1-3=0,300$

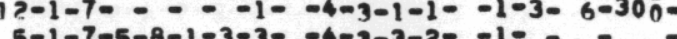
$5-1-7-5-8-1-3-3-4-4-3-3-2--1-3-6$
$12-1-1-800-$ $12-1-1-1-7-1-2-2-3-4-3-2-2-1-3-6-800$
$11-1-1-4-7-1-2-2-3-4-3-2-2-1-1-$ $11-1-1-4-7-1-2-2-3-4-3-2-2--1-1=:=$

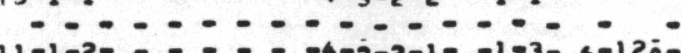
$11-1-2-0-8-120$ $6-1-1-6-8-4-3-4-2-6-3-1-1=-1=$ 10-1-6-5-8-3-3-4-2-4-3-2-1=-1-3-

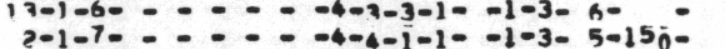
$12-1-7-5-7-3-3-1--4-5-2-1--1---$ $15-1-7-5-7-3-3-1=-4-2-2-1--10$ $6-1-7-5-7-2-2-1=-4-2-2-1=-1-1=$
$12-1-7-7-3-2-1=-4-2-2-1=-1-1=$

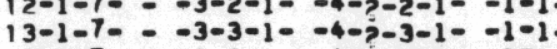

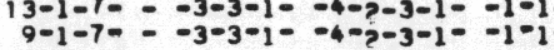

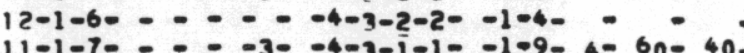
$11-1-7-2-0-3-4-3-1-1--1-9-4-60-40$ $11-1-7=-10=-1-2-4-2-3-1--1-1-$ $12-1-7-0-1-2-1--4-3-2-2--1-5$ 6-1-6-5-6-1-2-2- $-4-2-3-1--1-$ $7-1-6-5-7-1-2-2-4-4-2-2-1--1=-$
$12-1-6-5-6-1-2-2-4-4-3-1=-1=-$ $7-1-6--3-3-1--4-p-3-1--1-1$ $6-1-6--1-4-2-3-1--1-1=$ $9-1-6-5-6-2-3-1--4-2-2-1--1-=$
$15-1-6-5-6-2-2-2--4-2-2-1--1-$ $15-1-6-5-6-2-2-2--4-2-2-1--1-1-$
$13-1-6--2-2-1--4-2-2-2--1-1$ $13-1-6--2-2-1--4-2-2-2--1-1-$
$0-1-1-1-0-1-4-3-2-1--10$ $10-1-7-\ldots .--4-3-1-1--1-2$

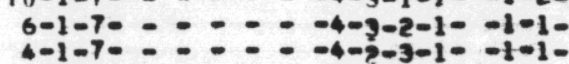
$6-1-7-\ldots-0 .-4-3-2-1--1-1$ $4-1-7-\cdots--4-3-2-1--1-1$ $7-1=7-==---4-3-2=1--1-4$ $3-1-7=-2=-8-3-2-1=-1-4-6-210=90$ $1-1-6-0--4-3-2-2--1-4-$ $1>-1-1-5-8-3-3-4-2-4-4-2-2--1=-$
$9-1-6---4-3-1-2-4-2-1-2-1-$ 9-1-6- - - -4-3-1-2-4-3-1-2--1$15-1-1=-1-3-2--4-4-1-1--1=-$
$10-1-1-=-1-1-4--4-4-1-2--1=-$
2.79

1.59

0.72

9.10

8.68

2.79

0.15

1.93

1.09

1.99

0.76

0.86

2.11

2.46

3.65

0.35

0.47

3.65

3.22

2.99

0.10

0.16

0.61

$7.4 ?$

2.70

6.56

1.20

4.55

5.48

$0.7 \mathrm{i}$

0.58

5.01

0.70

1.14

3.68

1.25

0.10

2.60

1.00

0.10

0.10

0.99

2.32

0.82

5.50
2.67 
LASL Uranium Hydrogeochemical and Stream Sediment Reconnaissance Data Listing

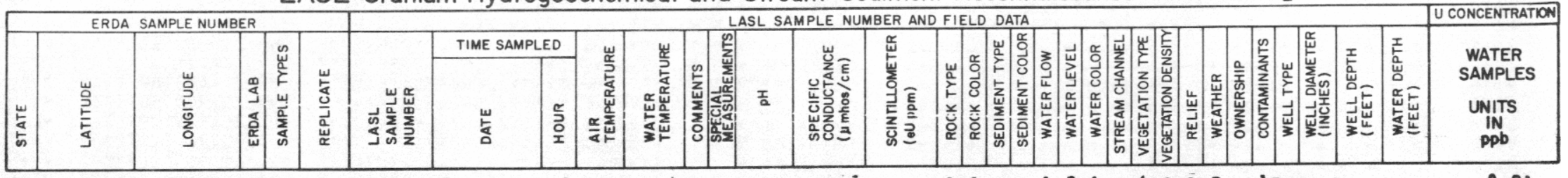

$56-44.7433-107.0277-2-10-0-201307-05 / 14 / 7 h-9-22-16.0^{-0} \mathrm{C}-0.8-250_{-}-$ $56-4.0 .2183-105.4361-2-08-$ $36-44.1025-105.068 n-2-08-$ $56-44.1005-105.1061-2-c 8-$ $56-44.0750-105.1052-2-68$ $56-44.0538-10^{5} .0933-2-08$ $36-44.0988-105.1700-2-08$ $50-44.0963-105.1988-2-08-$ $36-44.0866-165.225 n-2-08$ $56-44.4966-1 c 5.8566-2-C 3-$ $36-44.4858-105.9044-2-08-$ $56-44.4819-105.9047-2-08-$ $56-44.4688-105.8877-7-08$ $56-44.4577-105.618 n-2-00-$ $56-44.4875-105.7991-2-08$ $56-44.4202-105-7897-2-08-$ $56-44.4250-10=.8572-2-08$ $56-44.6508-165.1127-2-66-$ $56-44.6344-105.1102-2-06-$ $56-44.5016-105.2333-2-08-$ $56-44.5188-105 \cdot 2450-?-08$ $56-44.5419-105 \cdot 2083-2-08$ $56-44.5113-105.1611-2-C 6-$
$56-44.5130-105.1394-2-08$. $56-44.5611-105.1866-2-08-$ $56-44.5619-105.1333-2-04-$ $36-44.5927-105.1402-2-08=$ $56=44.5511-165.227 ?-2-04-$ $56-44.5733-105.2433-2-08$ $56-44.6022-105.1755-2-08$ $56-44.6094-105.2188-2-08-$ $36-44.1463-105 \cdot 1361-2-10-$ $56=44.0980-105 \cdot 1400-2-08-$ $56-44.0344-105.1405-2=08-$ $36-44.0650-105 \cdot 1627-2-10$ $56-44.0452-105.0355-2-04$ $56-44.0072-105.0066-2-08$ $56-44.1002-105.038 n-2-08$ $55-43.6652-107.0000-2-10=$ $56-43.6522-107.0355-2-09$ $56-43.6477-107.0361-2-08-$ $50-43.6444-107.053 n-2-06-$ $56-43.6594-107.0544-2-06=$ $56-43.6638-107.068 n-2-c^{7}=$ $56=43.6647-107.0688-2=07=$ $56-43.6877-107.1211-2-06$ $56-43.6661-107.1094-2-06=$ $56-43.6766-107.0827-2=06$ $56=43.6544-107.067 ?-2-06$ $56-43.6288-107 \cdot C>88-2-06$ $56-43.6127-107 \cdot c 61 b-2-06-$ $n=>01313-07 / 23 / 76-15-31-16 . n^{\circ}=-6.4-15 n^{\circ}-$ $9-201315-0713417 n-15030-16 \cdot n=-6.4016 n 0-$

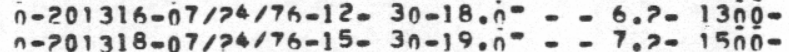
$0-201319-n 7 / 24 / 7 n-14-29-18.0^{-}-6.4-130^{-0}-$ $0-201321-07 / 24 / 79-16-29-12 . n^{-}-0.4-40^{0}-$

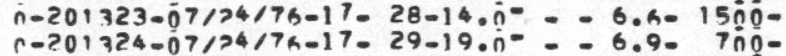

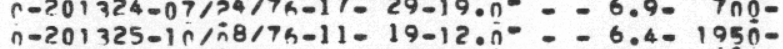

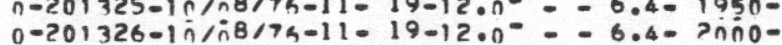

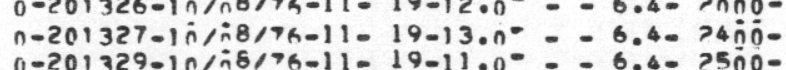
$0-201329-1 n / i 8 / 76-11-19-11 \cdot 0^{\circ}=-6.4-25 n 0^{-}$
$n-201332-1 n / i 8 / 7 n-12-2 n-13.0^{\circ}=-6.9-7 n n^{-}$

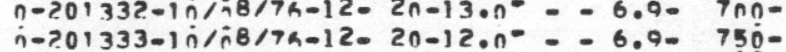
$n \rightarrow 01335-1 n / 88 / 76-12-2 n-14 \cdot n^{-}=-6.9-80^{-}-$ $n-201338-10 / n 8 / 7 a-13-21-13 \cdot n--6.9-7 n o-$

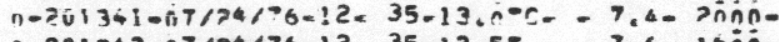
$n-201343-07 / 24 / 75-12-35-13.5^{\circ}-$ - 7.6- $16 n^{\circ} 0^{-}$ $0-201344-07 / 24 / 76-13-35-14.5-\mathrm{C}-$ - 7.4- $120.00-$ $n=>01345-n 7 / 24 / 76-13-35-13 \cdot \hat{n}-C_{-}-7.4-1 n i g-$ $n-201346-07 / 24 / 74-140-36-12 . n^{\circ} \mathrm{C}-{ }_{-}-7.9-11 n 0-$

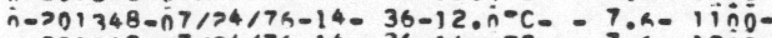

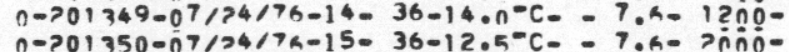
$0-201351-07 / 24 / 75-15-36-12 . n-C_{-}-7.6-16 n 0-$ $n=201352-07 / 24 / 7 h-16=36-13.5-C-7.2-17 n 00-$ $n-201353-07 / 24 / 7 h-16-36-12.5-c_{-}-7.4-11 n 0-$ $n \rightarrow 201354-07 / 24 / 7 b-17-35-13 . n_{-1}^{-C}-C_{-}+R_{-}-1100-$ $0-201356-07 / 24 / 75-18-35-14 . n^{-C} C_{-}-7 . h-160_{0}-0_{-}$ $n=201357-07 / 24 / 7 a-18-35-12.5^{-C}-c_{-}-7.4-12 n n-$ $n-201366-07 / 24 / 7 n-14-38-25 . n^{-}-0.3-230^{\circ}$ $n-201371-07 / 26 / 76-12-3 n-21 \cdot n^{-}-T_{-2}-1150-$ $n-201373-07 />6 / 7 h-12-31-13 . n^{\circ}-0.9 .9-950-$ $n-201375-n 7 / 26 / 7 n-13-32-25 \cdot n^{-2}=6.4-43 n n^{-}-$ $n-201980-07 / 26 / 7 h-15-27-18 \cdot r^{-}-0.20$ ? $2100-$ $n \rightarrow>01381-07 / 26 / 79-15-27-17 . n_{-}-0 . \hat{n}-19 n \hat{n}-$ $n \rightarrow>01382-07 />6 / 7 a-15-26-17 \cdot n^{-}=-6 . n-28 n 0^{-}$

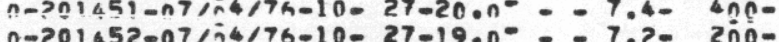

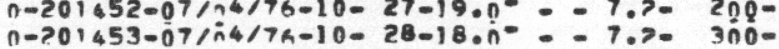

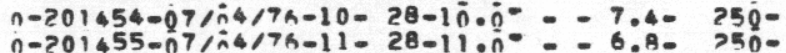
$n-201456-07 / n 4 / 76-11-28-15 . n^{\circ}=-7.2-250-$ $n-201457-n 7 / 14 / 7 n-11-28-11 \cdot n^{\circ}=-6 \cdot R-3 n 0-$ $n-201458-07 / 24 / 75-12-28-15 . n^{\circ}=-6 . A_{-}-2 n^{\circ}-$ $n=201459-07 / 24 / 7 n-12-28-150 n^{-}=-7.2-3 n 0-$ $n=701460-n 7 / 24774-12-28-19.0=-7.7-15 n-$ $10-201461-07 / 74 / 76-12-29-19 . n^{2}-0.8-300-$ $n-201462-n 7 / 34 / 7 a-10-3 n-10 \cdot n^{-}-6 \cdot 8-2 n 0^{\circ}$ $0-201463-07 / 84 / 7 n-14-31-17.0^{-}-7_{0}-5 n 0^{-}$
$10-1-1---1-3-1--4-3-1-2--1-$ $4-1-60=-0-4-3-1-1--1-1-$ $3-1-6-\ldots-0-3-2-1--1-1-$

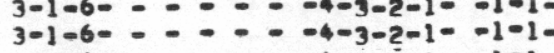
$3-1-6-\ldots-0-3-1-1--1-1-$ $1-1-6=-20=-0-7-3-2-1=-1-10$

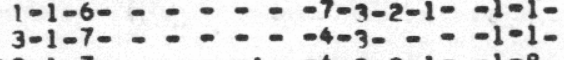
$12-1-7-\cdots--1 \div-4-3-2-1 \div-1-8$ $11-1-7-7=-1--4-3-2-1--1-1-$
$14-1-70-0-1--4-3-2-1--1-1-$ $8-1-7-\ldots--1<-4-3-2-1--1-1-$

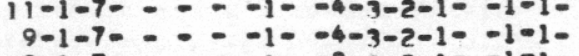

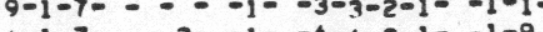

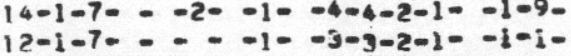
$14-1-7---2--1=-4-2-2-1--1-9-$

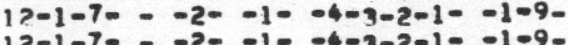
$6-1-7-=-2--1=-4-3-5-1=-1-9$ $0-1-7=-2=-1--4-4-2-1=-1-9$ $10-1-7---2--1--2-3-3-1--1-9-$ $11-1-7---2--1--3-3-3-1--1-9-$

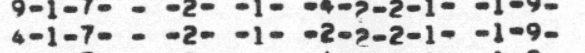
$11-1-7---2--1--4-3-2-1--1-9-$ $3-1-7---2--1--3-3-2-1--1-9-$
$12-1-6-5-6-2-2-2--4-2-2-1--1--$

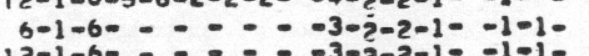
$15-1-6-10-0-3-2-5-1--1-1=$

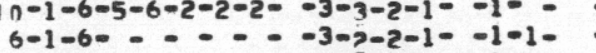
6-1-6- - . - - - -3-क- - $-1-1=-1-1-$ $6-1-6-0-3-2-2-1--1-1-$ $8-1-7-5-6-2-2-4--4-3-3-1--1-=$
$16-1-7-4-7-3-3-1--4-3-3-1-1-1=$ $16-1-7-4-7-3-3-1--4-3-3-1--1-1=$
$11-1-7--2-3-1--4-3-3-1--1-1=$ $16-1-7-5-6-3-3-1=-4-3-3-1--1=$
$9-1-7-5-6-2-2-1=-4-3-3-1--1=$ $11-1-7-5-6-3-3-1--4-3-3-1--10-$ $11-1-7-4-6-3-3-1--4-3-3-1--1=-$ $12-1-7-5-6-3-3-1--4-3-3-1--10=$
$>2-1-7-5-6-3-3-1--4-3-3-1=-1-$ $4-1-7-5-6-3-3-1--4-7-3-1--1=-$
$6-1-7-5-6-2-3-1--4-3-3-1--1-$

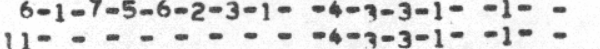
$16-1-7--2-3-1--4-3-3-1--1-7-$ 
LASL Uranium Hydrogeochemical and Stream Sediment Reconnaissance Data Listing

\begin{tabular}{|c|c|c|c|c|c|c|c|c|c|c|c|c|c|c|c|c|c|c|c|c|c|c|c|c|c|c|c|c|c|c|c|c|}
\hline \multirow[b]{3}{*}{ 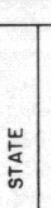 } & \multicolumn{5}{|c|}{ ERDA SAMPLE NUMBER } & \multirow{2}{*}{\multicolumn{26}{|c|}{ LASL SAMPLE NUMBER AND FIELD DATA }} & \multirow{3}{*}{\begin{tabular}{|c|} 
U CONCENTRATION \\
WATER \\
SAMPLES \\
UNITS \\
PDb \\
PDD
\end{tabular}} \\
\hline & & & & of & & & & & & & & & & & & & & & & & & & & & & & & & & & & \\
\hline & 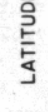 & $\begin{array}{l}\frac{3}{5} \\
\frac{1}{0} \\
\text { g. }\end{array}$ & 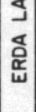 & 崩 & $\frac{\mathbb{d}}{\frac{d}{a}}$ & 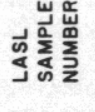 & 岁 & 홈 & 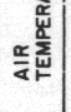 & 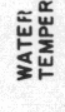 & 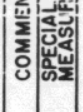 & $\bar{z}$ & 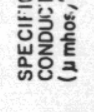 & 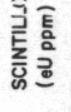 & 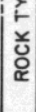 & 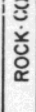 & & 嵓 & $\begin{array}{l}\frac{1}{\alpha} \\
\frac{a}{4} \\
\frac{5}{3} \\
3\end{array}$ & $\begin{array}{l}-\bar{x} \\
\underline{w} \\
\frac{5}{3} \\
3\end{array}$ & $\begin{array}{l}0 \\
\frac{\pi}{4} \\
\end{array}$ & 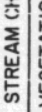 & 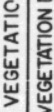 & $\stackrel{\underline{u}}{\underline{u}}$ & 竞 & 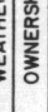 & 位 & $\mid \begin{array}{l}F \\
- \\
\frac{\mathrm{w}}{3} \\
3\end{array}$ & 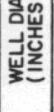 & 总 & $\mid \begin{array}{l}\text { 辞点 } \\
\text { 㫐 } \\
\text { 出 }\end{array}$ & \\
\hline
\end{tabular}

$56-43.6100-107.0666-2-06-$ $36-43.6227-107.0794-2-06-$ $56-43.6222-107.0622-2-06-$ $56-43.6383-107.0850-2-06-$
$56-43.6352-107.1150-2-06=$ $56-43.6722-107.0200-2-10-$ $56-43.6722-107.020 n-2-10-$ $56-43.6788-107 \cdot 0338-2-06-$ $56-43,7055-106.7777-2-10-$
$56-43,7000-106.7763-2-06-$ $56-43.7388-106.7791-2-10-$ $56-43.6888-106.7791-2-16-$ $36-43.6958-106.7916-2-06-$ $56-43.6958-106.7916-2-06-$
$56-43.6458-106.7461-2-10-$ $56-43.5958-106.8527-2-06$ $56-43.5875-106.8416-2-06-$ $56-43.5768-164.8375-2-16-$ $56-43.5722-104.8875-2-08-$ $56-43.6208-106.9222-2-06-$

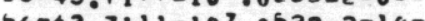
$56-43.7111-107.0522-2-10-$ $36-43.7200-107.050-2-060$ $56-43.7583-106.9341-2=07$ $56-43.7583-106.9541-2-07-$ $56-43.7375-106.9388-2=08$ 56-43.6833-10र. $111-2-00-$ 56-43.6680-106. $8166-2-10-$ 56-43.9961-10k. $7172-2-07-$ $56-43.9944-10 k .7625-2-0 \%$ $56-43.9625-104.7541-2-01=$ $56-43.8930-106.8361-2-06-$ $36-43.9125-106.8444-2-06-$ $56-43.9055-106.8055-2-06-$ $56-43.8527-106.7777-2-10-$
$56-43.5305-106.8625-2-07-$ $56-43.5305-106.8625-2-0 \%=$ $56-43.5319-106.8666-2-07-$
$56-43.5069-106.9583-2-08-$ $56-43.5027-106.9208-2-08-$ 56-43.5255-107.0311-2-06$56-43.5583-107.0377-2-06-$ $56-43.5430-107.0613-2-06=$ $56-43.5305-107.0727-2-06-$ $56-43.5294-107.0761-2-07-$ $56-43.5561-107.0888-2-06=$ $36-43.5400-107,1100-2-0 \%$ $56-43.5702-107 \cdot 0894-2-00-$ $56-43.5716-107.115 n-7-06-$ $56-43.5950-107,1044-2-06-$ $56-43.7633-167.1113-2-66-$ $56-43.7844-107.0527-2-06-$ $56-43,7811-107.1183-2-06=$ $56-43.7938-107.1033-?-06-$ $n-201464-n 7 / i 4 / 74-14-31-11 \cdot \bar{n}-=-6.8-450-$ $0-201465-07 / 24 / 76-14-31-9.0--0^{-7.2-} 200-$ $0-201467-07 / 14 / 75-16-32-18,00:-7.4=150-$ $0-201468-07 / \hat{n} 4 / 74-14032-15.0^{-}=-7.3-2 n 0^{-}$ $n-201469-07 / i 4 / 7 h-18$ - 32-13.ñ- - - 6.3- 35ñ $0-201470-07 / n 4 / 76-18=32-11, n-=-6, R_{-} 3 n_{0}-$

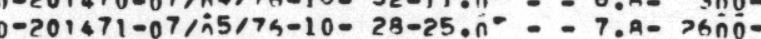

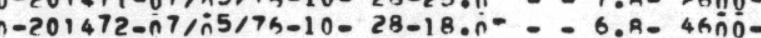

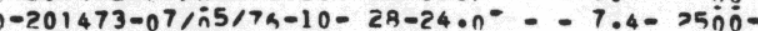
$0-201475-07 / 25 / 76-11-29-25.0^{-}-0^{-6.8}-11 n^{-0}$ n-201476-07/i5/76-11- 29-19.n:- - 6.R- 50̄ 0 作 (201480-07)

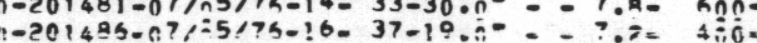
$n-201489-07 / n 5 / 7 n-16-35-19.0^{-}-0.8-300-$ $11.00-=600-550-$ $0-201494-07 / i 8 / 7 a-10-30-23 . n^{-}-0.6-200-$ - 0149507 her 201496-07/28 1->01498-n>tis - $01499-077877 h-12032-170 n=-6.90 \quad 370$ $0-201504-07148742-20-28-26.0--6.9-$ anno-

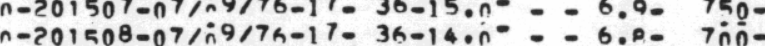
$n \rightarrow 01510-07 / i 9 / 7 n-18-36-19.0-=7.4080^{-}$ $n-201513-n>1 / 29 / 7 n-20-27-16.0^{\circ}-8_{-5}-7 n^{-}$ $n-201514-07 / i 917 n-20-27-19.00^{-}=8.205 n n^{-}$ n-201515-n7/i9/7n-20-27-18,n-:-8.i- 55j$n-201520-07 / i 9 / 7 h-21-25-21 . n-=8.2019000-$ n-201522-07/i0/7a- 1- 18-15.n- - - 6.R- 11 innn-201523-07/1017k- 7- 18-15 on- - - 6.8- ling-

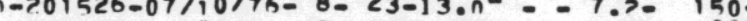
n-201527-0711017he-8. 23-14.n- - - 7.40 2 no n-201529-07/1017h- 9- 27-13.0.- - 7.2- $79 \mathrm{k}$ $n-201531-07 / 10176-9-27-12 . n=-6.9-250$ $0-201532-07 / 10 / 7 a-10-28-8 . n^{-1}--6.50$ ThO $n-201533-07 / 70 / 7 h-1 n-28-6 . n^{-}-6_{0}-2-240-$ $n-201534-07 / 1017 h-10=28-15 \cdot n^{-}=-6 . h-3 n \rho-$ $n \rightarrow 01535-07 / 1017 a-11-31-13 \cdot n^{-}-6.8-350-$ n-701536-07/70176-11- 31-14.00= - 7.4- 310$n-201537-07 / 10 / 7 a-11-31-11 \cdot n=-6.3-3 n n-$

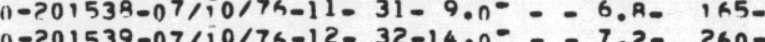
$n-201539-07 / 10 / 74-12-32-14 \cdot 0 n^{-}-7.2-26 n-$ $n-201540-07 / 10 / 7 h-13-35-17.0-0-6.8-150-$ n-201541-07/io/7h-13-35-15.n- - - 6.9- $2 n 0-$ $n-201543-07 / 1017 n-14-35-12 . n-=-5.8-50-$
$12-1-7---3-3-1--4-3-3-1--1-=$ $12-1-7---3-1-1--4-3-3-1--1=-$ $11-1-7-5-2-3-3-1=-4-3-3-1=-1<=$ $16-1-7-5-6-3-3-1=-4-3-3-1=-1=$ $8-1-7-5-6-3-3-1--4-3-3-1--10$ $8-1-7-5-6-3-3-1=-4-3-3-1=-1-$ $11-1-7-5-6-2-3-2-0-4-3-3-1--1-=$ $1-1-7-5-6-3-3-1--4-3-3-1--1-=$ $8-1-7-5-6-2-3-2--4-3-3-1=-1-:$ $12-1-7-5-6-3-3-2--4-3-3-1--1-$ $9-1-7-0-3-3-1--4-3-3-1--10=$

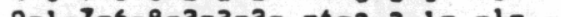
$14-1-7-6-8-2=2-20-4-3-3-1=-1-$ $16-1-7-5-6-2-2-3--4-3-3-1--1--1$

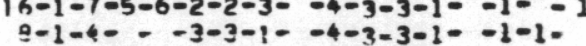
$6-1-4-4-6-3-3-1--4-7-3-1--1-$ $0,1-7-5-6-2-3-5-(-4-3-3-1=-10=$ $9-1-7-5-6-2-3-2=-4-3-3-1=-1=$ $9-1-7-5-6-3-3-1=-4-3-3-1=-1-$ : $9-1-4--3-3-1--4-3-3-1--1-$ $11-1-4=-3-3-1--4-3-3-1--1-3-$ - $1-1-4-0-3-3-10-4-3-3-1-1-3-$ $11-1-4-5-7-3-3-10-4-9-2-1--10-$ $8-1-7-5-6-3-3-1=-4-3-2-1--1=$ $11-1-7-5-6-3-3-1=-4-3-2-1=-1=$ B-1-7-5-6-3-3-1- -4-3-3-1- -1- $11-1-7-5-6-3-3-1--4-3-3-1--1=$ $9-1-7-5-6-3-3-1--4-3-3-1--1-$ $11-1-7-6-8-3-3-1--4-3-2-1--1=$
$3-1-4-5-4-3-3-1--4-3-2-1--1-$ $3-1-4-5-4-3-3-1--4-3-2-1--1-$
$3-1-4-5-4-3-3-1--4-3-2-1--1-$ $16-1-7---3-3-1--4-3-2-1--1-3-$ $12-1-7--3-3-1--4-3-2-1--1-1-$
$16-1-4-5-6-3-3-1--4-3-2-1--10$ $16-1-4-5-6-3-3-1=-4-3-2-1=-10$
$14-1-4-5-6-3-3-1=-4-3-2-1=-1=$
$11-1-4-5-6-3-3-1=-4-3-2-1=-1=$ $6-1-4-5-6-3-3-1--4-3-2-1<-1-0$ $6-1-4-5-6-3-3-1=-4-3-2-1--10$
$1 \geq-1-4-5-6-3-3-1=-4-3-3-1--10$ $12-1-4-5-6-3-3-1--4-3-3-1--1<-$
$12-1-4-5-6-3-3-1--4-3-2-1--1<-$ $11-1-4-5-6-3-3-1--4-3-3-1--1=$ $9-1-4-5-6-3-3-1--4-3-4-1--1=-$
$9-1-7-5-6-3-3-1--4-3-4-1--1-$ $16-1-7-5-6-3-3-1--4-3-4-1--1-=$ $14-1-7-5-6-3-3-1=-4-3-4-1--1=-$ $16-1-7-6-8-3-3-1--4-3-4-1--1=-$
$9-1-7--3-3-1--4-3-4-1--1=$
1.38

0.45

0.87

0.78

0.56

0.10

8

01

2.74

5.11

6.44

1.2

1.81

-

0.10

.

1.10

0.1

0.10

0.29

0.55

0.36

1.35

0.58

1.49

.

1.21

.? 7

3.71

2.83

.49

6.71

5.53

0.21

0.16

0.21

0.19

0.60

0.10

0.10
0.87

0.52

0.61

0.10

1.07

0.10

$0.1 ?$

0.10 
LASL Uranium Hydrogeochemical and Stream Sediment Reconnaissance Data Listing

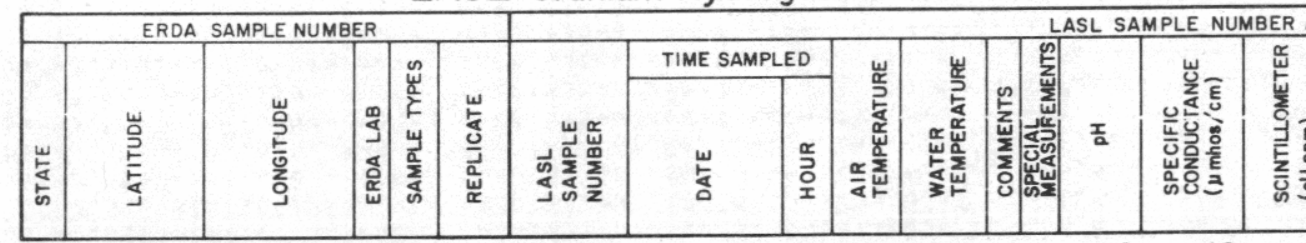

$56-43,8044-107,1113-2-06-$ $56-43.8238-107.0888-2-06-$ $56-43.8133-107.0488-2-67-$ $56-43.8543-107.0961-2-06-$ $36-43.8402-167.0738-2-16-$ $56-43.8794-107.1083-2-06-$ $36-43.8838-107.080 n-2-10-$ $56-43.8977-107.0766-2-06-$ $56-43.9083-107.0283-2-n 7=$ 56-43.01 $38-10 \mathrm{r}$. $56-43.8277-10 \mathrm{r} .825 \mathrm{c}-2-006$ $56-44.2416-106.9633-2-07-$ $36-44.1894-106.9100-2-07-$ $56-44 \cdot 1855-107 \cdot 0119-2-c 7-$ $56-44.1711-107 \cdot 0119-2-06-$ $56-44 \cdot 1600-107.0227-2-07-$ $35-64.1325-107.0043-2-06-$ $56-44.1322-107 \cdot 1205-2-07-$ $56-44.1061-107.1100-2-07-$ $56-44.0977-107.0938-2-n 7-$ $56-44.0677-107 \cdot 1072-2-07-$ $56-44.0311-107.105 ?-7-166-$ $56-44.0172-107.0938-2-06-$ $56-44.0255-107.0222-2-07-$ $56-44.0133-106.9936-7-07=$ $56-44.01111-106.97 n 5-7-06-$ $56-44.0144-1 c 6.9483-2-06-$ $56-44.0177-106.9244-2-06=$ $36-44.0272-10 \mathrm{H} .8955-2-06-$ $56=44.0238-106.8683-2-06-$ $56=44.0277-104.8627-2-06-$ $56-44.0438-106.8505-2-06-$ $56-44.0305-106.9261-2-07=$ $56-44.0313-106.9255-2-07-$ $56-44.0694-106.9005-2-07-$ $56-44.0827-106.8419-2-06-$ $56-44.0827-1$ UR. $9055-2-06$ $56-44.0927-106.8116-2-06-$ $56-44.0177-107.0527-2-07=$ $56-44.0038-107.0705-7-10-$ $56-43.9872-107.6366-2-16=$ $56-43.9788-107.0594-2-16-$ $56-43,9805-107,1194-2-06-$ $56-43.9783-107.0838-2-10-$ $26-43.9616-107.0744-7-10-$ $56-43.9511-107.0572-2-10-$ $56=43.9555-107.0327-2-06-$ $56-43.9583-1 C^{7} .0911-2-06-$ $56-43.9238-107.0588-2-06-$

\section{$0-201544-07 / 10 / 7 n-14-35-10 \cdot n^{-}=-5.7$} n-201545-07/i0/74-14-36-12.0- - 5.R- 4ñח-201546-07ri0 $n-201548-07 / 10 / 74-15-35-9 . n^{-}-5.5-50-$ $n \rightarrow>01549-07 / 1017 k-15-35-13 . n^{-}=-6 . h-350-$ $0-201550-07 / 10 / 76-15-35-21 . n^{-}-0_{0}-6-250-$

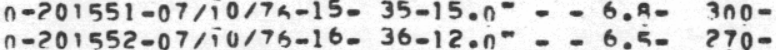
$n=201552-07 / 10 / 76-16-36-12 \cdot n=-: 6.5-270-$
$n \rightarrow 001554-07 / 10 / 74-17-37-18.0-0$ $n-201557-07 / 1017 h_{-17}-37-26 . n^{-}=-6.9-26 n 0-$

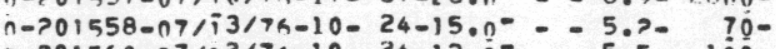

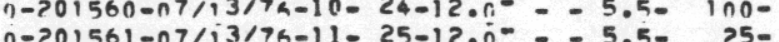
$n=201561-n 7 / 13 / 76-11-25-12 \cdot n^{-1}=-5.5-\quad 25-$ $n-? 01563-n 7 / ; 3 / 7 a-11-25-16 . n^{-}=-5.5-30^{\circ}-$ n-P01564-n7/i3/76-i2- 26-13.0. - - 5.2- 50-

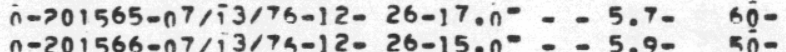
n-201567-07/36-13-26-17.0-: - 5.7- 40$0-201568-07 / 337 A^{-13}-26-18 . n^{-}-5.7-50-$ $0-201569-07,13 / 74-140-29-2 n 0_{0} 0^{-}-5.90$ $n-20157 n-07 / 13 / 76-14-29-11.0-0-5.0-5 n-$ $n-201571-n 7 / 13 / 7 h-15-29-18 \cdot 0^{-}-5-5.7-60-$ $n \rightarrow 01572-07 / 13 / 7 h-15-29-19 \cdot n=-5.9-70-$ $n \rightarrow 01574-071,3376=10-23-2000-=0.30$ 90$n-701575-07 / 13 / 75-17-23-13, n_{-}=-5.9-$ $n-201576-n 7 / 13 / 7 n-17-23-14 \cdot n^{-}-5=5.9-$
$n-201577-07 / 13 / 7 n-17-23-12 \cdot 0^{-}-0-$ $n-201578-07 / 13 / 7 n-17-23-14 \cdot n^{-}-5.9090-$ $n-201579-07 / 13 / 7 h-17-23-12.00=-5.90 \quad 60-$ $n \rightarrow 01581-07 / 13 / 7 n-18=23-15 . n^{-}=-5.9-60-$ $n \rightarrow 201583-07 / i 3 / 7 a-18-23-16 . n^{-}-$- 5.7- $70-$ $n-201584-07 / 13 / 7 h-18-23-14 \cdot n^{-}-5.9-50-$ n) $=201585-n 7 / i 3 / 7 a-19-22-13 . n-2-5.7-90-$

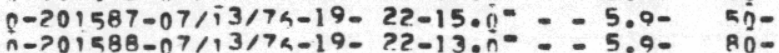
$1-201589-07 / 14 / 76-8-15-10.0^{\circ}--5.5=30-$ $0-201590-07 / 14 / 7 a-8015-12.0-0-6.7-i 50-$ $0-201591-07 / 14 / 76-8-15-9.00--6.3-270-$ $n \rightarrow 01593-n 7 / 14 / 7 a_{0}=9-2 n-12 . n^{-}=6.10300-$ $n \rightarrow>01594-n 7 / 14 / 76-9-2 n-13 . n^{-}-$- 6.20 3no$n \rightarrow 201595-07 / 14 / 76-9-20-11, n^{-}=-6.5-350-$
$n \rightarrow>01596-07 / 14 / 75-90$ $n=>01597-07 / 14 / 74-10-20-10, n=-0.504 n 0-$ $n=201598-07 / 14 / 7 k-10-2 n=9.0^{\circ}--6.1-2900$

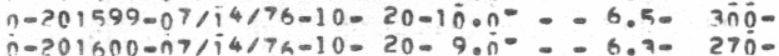

$12-1-7-5-6-3-3-1--4-3-4-1--1--=$ $6-1-7-5-4-3-3-1--4-3-4-1--1=-$ $3-1-7---3-3-1=-4-3-4-1=-1=$ $9-1-7-5-6-3-3-1--4-3-4-1=-1-$
$8-1-7-5-6-3-3-1--4-3-4-1--1-$ $8-1-7-5-6-3-3-1--4-3-4-1--1-1=$
$12-1-7-4-6-3-3-1--4-3-3-1--1<-$ $2-1-7-4-6-3-3-1--4-3-3-1=-1=-$
$16-1-7-5-6-3-3-1--4-3-4-1--1=-$ $3-1-7-5-6-3-3-1=-4-3-3-1=-1<-$ $12-1-7-5-6-3-3-1--4-3-4-1--1=$ $12-1-7-5-6-3-3-1=-4-3-4-1=-1=-$
$8-1-7-5-6-3-3-1=-4-3-3-1=-1=-$ $12-1-7-5-4-3-3-1=-4-3-3-1--2=$ $12-1-7-5-8-3-3-1=-4-3-4-2--1-=$ $3-1-7-4-6-3-3-1=-4-3-2-2--10=$ $4-1-7-4-6-3-3-1=-4-3-4-2--1=$ $4-1-7-4-6-3-3-1=-4-3-4-2-1-10$
$6-1-7-5-6-3-3-10-4-3-4-2--1-$ $4=1-7-4-6-3-3-1--4-3-4-2--1--$ $6-1-7-4-6-3-3-1=-4-3-4-2--1=-$ $9-1-7-5-6-3-3-1--4-3-4-1--1=$ $9-1-7-4-8-3-3-1<-4-3-4-1--1--$ $16-1-7-4-6-3-3-1=-4-3-2-1--1=$
$9-1-7--6-3-3-1=-4-3-4-1--1=$ $9-1-7--3-3-1=-4-3-4-1--1=$
$1-1-7-5-6-3-3-1--4-3-4-1--1=$ $2-1-7-5-6-3-3-1--4-3-4-1--1-=$ $8-1-7-5-6-3-3-1--4-3-4-1--1=-$ $6-1-7--3-3-1--4-3-4-2--1<-$ $9-1-7-5-6-3-3-1--4-3-4-1--1<-$ $8-1-7-5-6-3-3-1--4-3-4-1--1=$
$1-1-7-5-6-3-3-1=-4-3-4-2--1=$ $11-1-7-5-6-3-3-1--4-3-4-2--1=$
$8-1-7-5-6-3-3-1--4-3-4-2--1=$ $8-1-7-5-6-3-3-1=-4-3-4-1--10=$ $11-1-7-5-6-3-3-1=-4-3-4-1--1=-$
$9-1-7-4-8-3-3-1:-4-3-4-2--10$
$6-1-7-4-8-3-3-3-10$ $9-1-7-4-8-3-3-1=-4-3-4-2--1=-$
$6-1-7-4-8-3-3-10-4-3-4-2--1=$ $6-1-7-4-8-3-3-1-=4-3-4-2--1-$
$6-1-7-4-6-3-3-1--4-3-3-1--10=$ $6-1-7-4-6-3-3-1<-4-3-3-1--1<-$ $9-1-7-4-6-3-3-1=-4-3-4-1--10=$
$9-1-7-4-6-3-3-1=-4-3-4-1--1=$ $9-1-7-4-6-3-3-1=-4-3-4-1=-1=$
$3-1-7-4-6-3-3-1=-4-3-4-1--1=$ $6-1-7-4-6-3-3-1--4-3-4-1=-1=$ $9-1-7-5-6-3-3-1--4-3-4-1--1-$ $11-1-7-4-6-3-3-1--4-3-4-1--1=$ $12-1-7-4-6-3-3-1=-4-3-4-1--1=$ $8-1-7-5-6-3-3-1=-4-3-2-1=-1=$ $8-1-7-5-6-3-3-1--4-3-4-1--1=$
$9-1-7-5-6-3-3-1--4-3-4-1--1=$ $9-1-7-5-6-3-3-1--4-3-4-1--1=$ $6-1-7-5-6-3-3-1=-4-3-4-1=-1<:$
$11-1-7-5-6-3-3-1=-4-7-3-1=-1=$ $12-1-7-4-4-3-3-1=-4-3-4-1=-1=$
$11-1-7-5-6-3-3-10-4-3-4-1=-1=$ $9-1-7-5-6-3-3-1=-4-3-3-1=-1-=$ $14-1-7-5-6-3-3-1--4-3-4-1--1=$
WATER SAMPLES UNITS Ppb 
LASL Uranium Hydrogeochemical and Stream Sediment Reconnaissance Data Listing

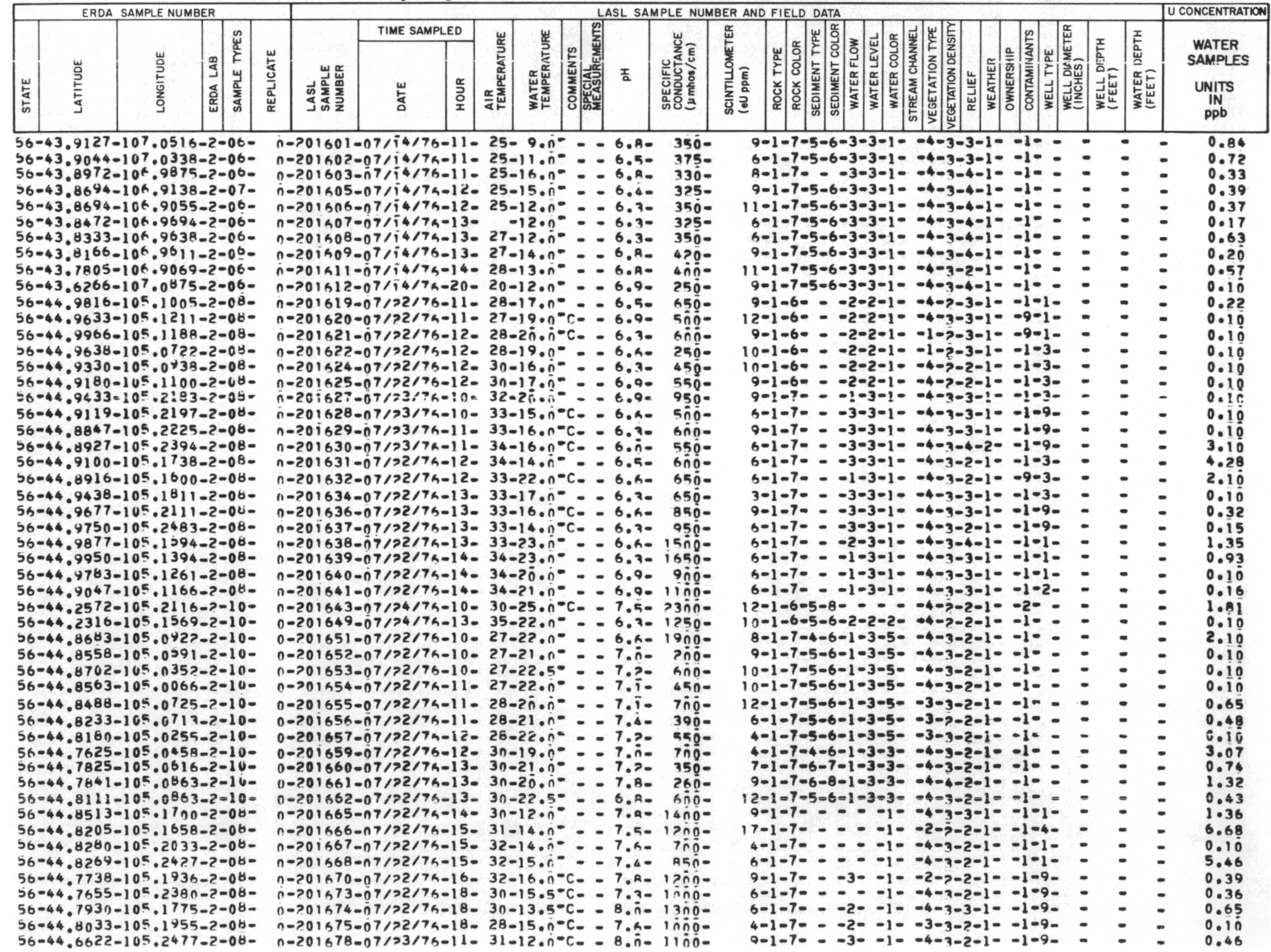


LASL Uranium Hydrogeochemical and Stream Sediment Reconnaissance Data Listing

\begin{tabular}{|c|c|c|c|c|c|c|c|c|c|c|c|c|c|c|c|c|c|c|c|c|c|c|c|c|c|c|}
\hline \multirow[b]{3}{*}{$\frac{5}{5}$} & \multirow{3}{*}{$\begin{array}{l}\text { ERDA } \\
\text { 㟧 } \\
\text { 兽 }\end{array}$} & \multirow[b]{3}{*}{$\begin{array}{l}\frac{\mathrm{u}}{2} \\
\frac{1}{0} \\
\frac{0}{0}\end{array}$} & \multirow{3}{*}{\multicolumn{2}{|c|}{ 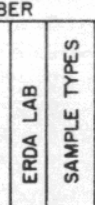 }} & \multirow[b]{3}{*}{ } & \multirow[b]{3}{*}{ 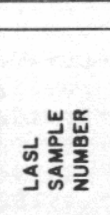 } & \multirow{2}{*}{\multicolumn{2}{|c|}{ TIME SAMPLED }} & \multirow{3}{*}{ 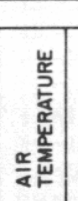 } & \multirow{3}{*}{ 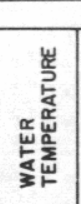 } & \multirow{3}{*}{ 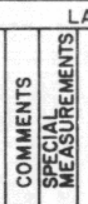 } & \multirow[b]{3}{*}{ I } & \multirow[b]{3}{*}{ 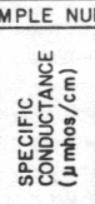 } & \multirow{3}{*}{ 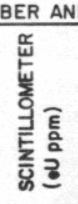 } & \multirow{3}{*}{ 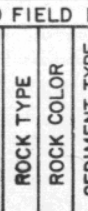 } & DATA & & \multirow{3}{*}{\multicolumn{2}{|c|}{ 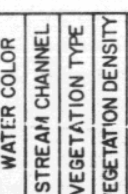 }} & & & & & & & 0 \\
\hline & & & & & & & & & & & & & & & & \multirow{2}{*}{ 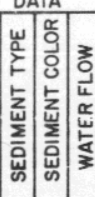 } & \multirow{2}{*}{ 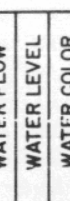 } & & & \multirow{2}{*}{\multicolumn{2}{|c|}{ 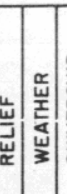 }} & \multirow{2}{*}{ 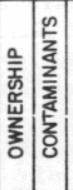 } & \multirow{2}{*}{ 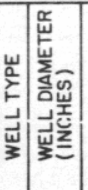 } & \multirow{2}{*}{ 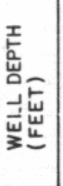 } & \multirow{2}{*}{ 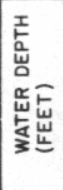 } & \multirow{2}{*}{$\begin{array}{c}\text { WATER } \\
\text { SAMPLES } \\
\text { UNITS } \\
\text { PpD } \\
\text { Ppb }\end{array}$} \\
\hline & & & & & & & 占 & 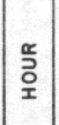 & & & & & & & & & & & & & & & & & & \\
\hline
\end{tabular}

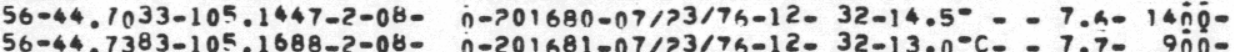

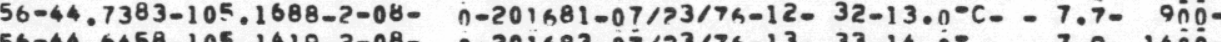
$56-44.6458-105.1419-2-08-\quad n-201683-07 / 23 / 76-13-33-14.0 n^{\circ}=-7.9-14 n 0-$ $56-44.6816-105.1686-2-08-n-201686-07723776-14-33-13.5-2-7.7-6 n 0-$ $56-44.7022-105.1241-2-08-0-201691-07 / 33 / 7 h-15-32-13.5-0.8 . n-19 n 0-$ $56-44.7016-105.0008-2-08-0-201696-07 / 33 / 76-17-30-14 \cdot 00=-8.5-15 n 0-$ $56-44.6772-105.0758-2-08-n-201699-07 / 74 / 7 a-11-34-11.00--7.4-2100-$ $56-44.6597-105.0666-2-08-\quad h-201700-07 / 74 / 74-11-35-1200=-07.40-2108-$ $56-44.1591-105.9144-2-08-\quad n-201705-10 / B 8 / 7 A-14-22-9.0-C=-6.5-680-$ $56-44.1488-105.9066-2-08-0-201707-10 / 38 / 7 a-14-22-1000-C-0.5-70 j-$

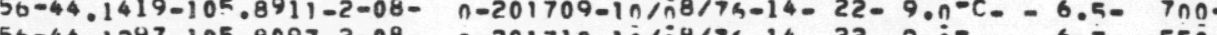

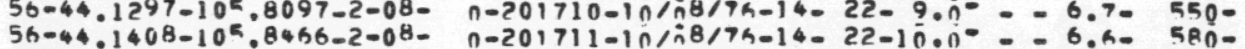
$5 B-44.1727-105.8688-2-08-n-201713-1 n / i 88 / 7 a-15-22-11 \cdot n-C=-6 . h-6 n O-$

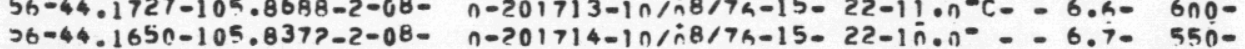

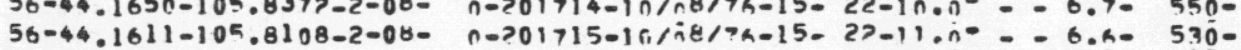

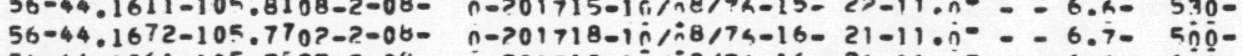
$56-44.1861-105.7527-2-08-n-201719-1 n / n 8 / 76-16-21-11 \cdot n=-6.6-6 n 0-$

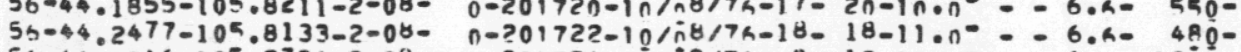
$56-44.1216-105.7736-2-08-n-201724-10 / 79175-8-12-1000=-0.4012 n 0^{-}$ $56-44.1227-105.7919-2-08-n-201725-10 / n 917 a-8-12-11 \cdot n_{0}^{-0}-0.4-130_{0-}^{-}$

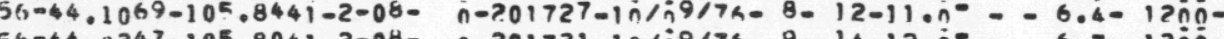
$56-44.0347-105.8041-2-08-n-201731-10 / 2917 n-9-14-12 . n=-6.7-13 n 0-$ $56-44.0133-105.7977-2-08-n=201734-1 n / n 972-9-14-12 \cdot n=-6.3-14 n 0-$

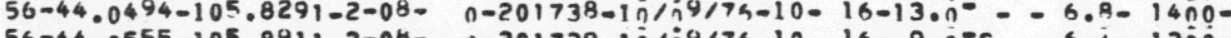

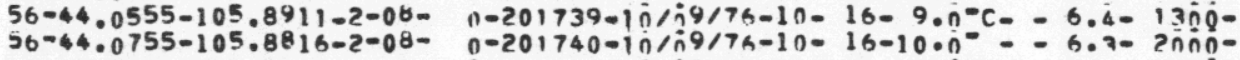
$56-44.0544-105.9063-2-08-$ $56-44.0758-105.9538-2-08-$ $56-44.0805-105.9791-2-08-$ $56-44.0577-105.9594-2-08-$ $56-44.0605-105.9730-2-08-$ $56-44.0436-105.9744-2-08-$ $56-44.0108-105.9605-2-08$ $56-44.0158-104.0083-2-108$ $56-44.0269-10 \% .0247-2-08$. $56-44.0038-106.0261-2-08-$ $56-44.0069-106.0566-2-08$ $56-44.0300-106.0530-2-08-$ $56-44.0650-106.0669-2-08=$ $36-44.0786-106.1294-2-108-$ $56-44.1033-10 \% \cdot 1483-2-08-$ $56-44 \cdot 1191-106,1263-2-08$ $55=44.1102-106.0 \times 38-2=08$ $56-44.1111-106.0005-2-08-$ $56-44.1000-106.0622-2-10-$ $56-44.0925-106.0169-2-08-$ $56-44.1291-106.0447-2-08-$

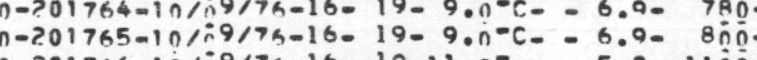
$0-2017 k h-1 n / 49 / 7 a-16-19-11 . n--5.8-11$ - $0=$ $n-201772-1 n / 10 / 7 h-8-12-9.0^{-}-6.9-1 n n \overline{0}-6$
$11-1-7-1--1--2-3-2-1=-1-3-$
$16-1-7--2-1--4-4-3-1--1-9-$

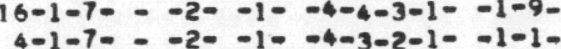
$4-1-7=-2-1-1--4-3-2-1--1-1-$
$9-1-70=-0-1--2-2-2-1--1-1=$ $9-1-7-0--1=-4-3-2-1--1-3-$ $8-1-7=--1=-3-3-2-1=-1-1=$ $8-1-7=-0-1=-4-3-2-1--1-1=$ $9-1-7-\ldots-1--4-3-2-1--1-1-$ $4-1-7-=-1--4-3-2-1--1-1-$ $14-1-7-\ldots--1--3-3-2-1=-1-9-$

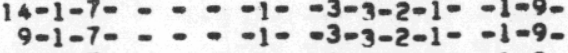
$14-1-7-0-1--4-3-2-1--1-8-$

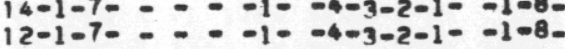
$13-1-7-0--1--4-3-2-1--1-9$ $14-1-7-\ldots--1--4-3-2-1--1-1-$ $16-1-7-1-1-1-4-3-2-1--1-1-$

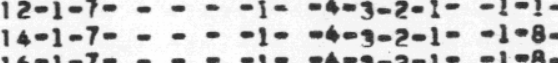
$16-1-70--10-4-3-2-1=-1-1=$ $12-1-7=-:-1--4-3-2-1--1-1=$ $14-1-7-=--1--4-3-2-1=-1-1=$ $16-1-7-0--1--4-3-2-1--1-1=$ $16-1-7-7=--1--4-3-2-1-1-1-1-1-$ $11-1-7-0--1--4-3-2-1--1-1-$ $14-1-7-7=-1--4-3-2-1--1-1-$ $11-1-7=0=0=1=-4-3-2-1=-1=-1-3=$ $12-1-7-=--1--4-3-2-1--1-9-$
$14-1-7-=-10-4-3-2-1--1-8-$ $16-1-7-=-1--4-3-2-1=-1-9-$ $14-1-7---1--3-3-2-1--1-8$ $16-1-7-0-1--4-3-2-1--1-9-$
$5-1-7-0-1--4-3-2-1--1-1-$ $3-1-7-1-0-1--4-3-2-1--1-1-$
$8-1-7-0-1-4-4-2-1--1-1-$ $8-1-7-0--1--4-3-2-1--1-1-$ $12-1-7-0-1--3-3-2-1=-1-1-$
$16-1-7-0=-1--3-3-2-1=-1-1-$ 9-1-7- - - - -1- -4-3-2-1- -1-1 $12-1-7-0-1--4-7-2-1--1-1-$
$9-1-7-7-0-1--4-3-2-1--1-1-$

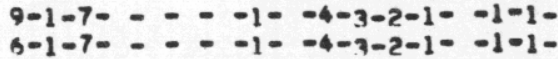
14-1-7- - - -1- -4-3-2-1- -1-9. $8-1-7-0:-1--4-3-2-1=-1-9-$
$16-1-70=-1--4-3-2-1=-1-9-$ $14-1-7-\ldots-1--4-3-2-1=-1-9$ $16-1-7---1--4-3-2-1--1-9$ $12-1-7-5-6-3-3-1-3-3-3-2-1=-1-$ $15-1-7-\div--1--4-3-2-1--1-9$
$8-1-7-1--1--4-3-2-1--1-1$ 0.35 0.50 0.26 0.26
5.23 5.23
2.26 2.26
0.67 $0.6 ?$ 1.17 0.000 0.22 0.63 0.10 0.18 0.10 0.51 0.10 0.10 0.100 0.15 0.58 2.01 0.91
1.61 1.61 2.79 2.19 4.66 0.75 0.0 0.10 0.76 2.24 0.56 1.83 2.24 0.56 1.64 0.10 0.10 2.32 1.92 1.97 0.10 0.10 0.33 0.18 0.10 0.84 0.100 
LASL Uranium Hydrogeochemical and Stream Sediment Reconnaissance Data Listing

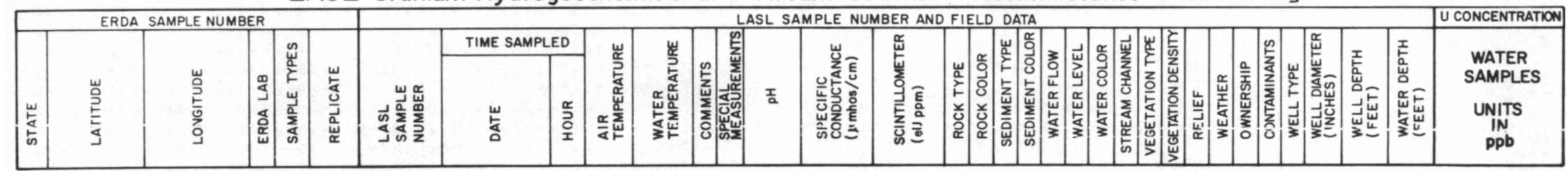

$56-44 \cdot 1427-106.0463-2-030 \quad n-201773-10 / 10 / 76-8-12-1000-0-6.8-1100-$

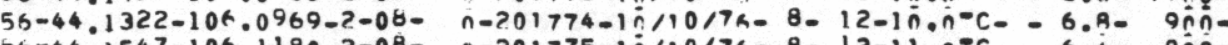

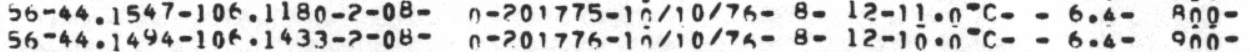
56-44.1747-106.1358-2-03- n-201777-10170/74-9-14-11.0.-C- - 6.4- 10n0$36-44.1744-106.1116-2-08-00-201778-1 n / 10 / 79-9-14-11 . \hat{n}-C_{-}-6.9-11 \hat{n} 0-$ $56-44.1902-106.1373-2-08-\quad n-201780-10 / 10 / 76-9-14-12.0-C-C-6.9-1 n 00-$

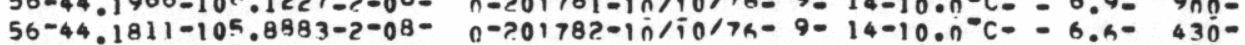

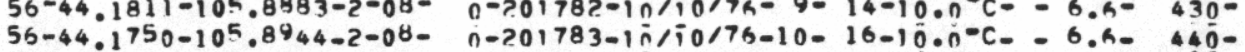

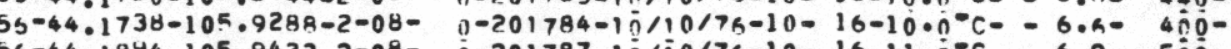

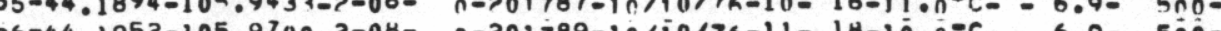

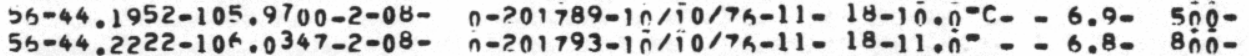

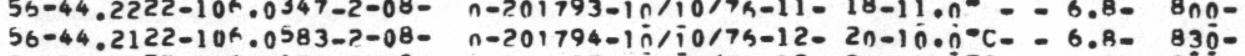
$55-44.2175-106.1175-2-08-0-701797-1 n / 10 / 7 a-12-20-11 \cdot n-C=-6.9-900-$

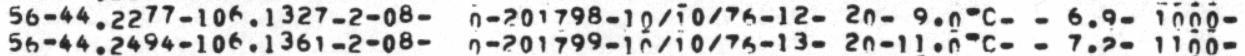

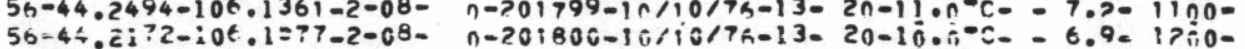

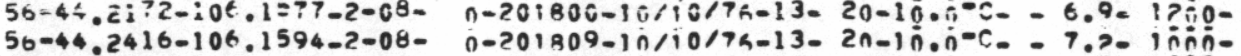

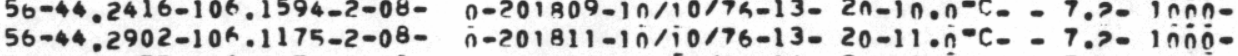
$56-44.2655-106.107 ?-2-08-$ n->01R12-1n/i0/7h-14- 20-9.0̈-C- - 7.2- 950-

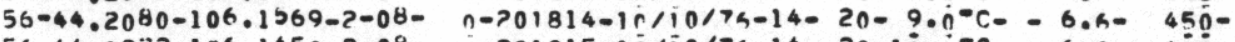
$56-44 \cdot 1922-106 \cdot 145 n-2-08-\quad n-201815-1 n / 10 / 7 n-14-2 n-1 n+n^{-} C-C-6.4-440-$

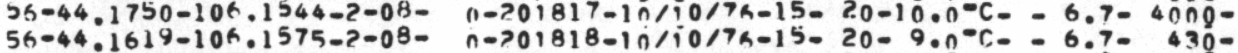

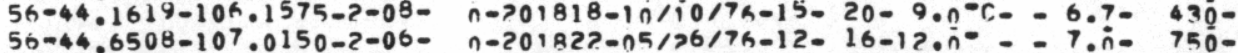
$5 h-44.6358-107.025 n-2-08-\quad n \rightarrow>01830-05 />66 / 7 n-12-18-12.5^{\circ} C-C-6.7-140-$ $50-44.6297-107.0280-2-07-n-7.01931-05 />6 / 75-13-19-7.0-05.9-4011-$ $56-44.6708-107.015 n-2-08-0-201834-05 / 76 / 7 h-14-22-14.01--2.8-8 n 0-$ 56-44.6691-107.0683-2-08- n-701835-05/76/76-14- 25-1n.n-C- - 7.ñ- 375-

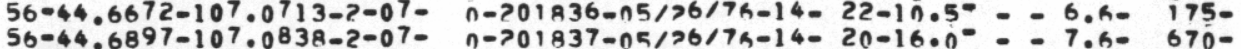
$56-44.7050-107.0780-2-08-j-201838-05 / 26 / 79-15-20-14 . \hat{n}^{-}=-6.8-60^{-}=$ $36-44.7333-107.0730-2-08-n-201840-05 / 36 / 75-16-22-20.0-1=6.8-850-$ 56-44.0408-106.7652-2-08- n->01842-05/>7/7h-1n-23-18.ñ-C- - 6.5- 45ñ56-44.0269-106.7655-2-07- n-?01843-05/77/76-11-23-12.0- - - 6.5- 265$56-44.0619-106.7544-2-10-0-201846-05 / 77 / 74-13-26-23 . n=-6.4-15 n 0-$ $56-44.0866-106.8025-2-06-0-701851-05 / 37 / 76-16-22-18.0-=-6.9-460-$ $56-44.0738-106.7955-2-06-n-201852-05 / 77 / 76-16-24-14.00=-6 . h-340-$

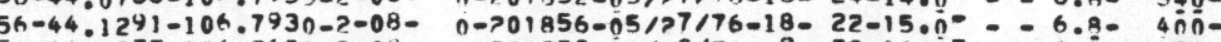
$56-44.1977-106.7691-2-08-n-701858-05 />8 / 78-9-23-160 n^{\circ}-0.60 n-154-$ 56-44.2163-10r.7583-2-08- n-201860-05/28/76-10- 25-14.5-C- - 6.h- 11no-

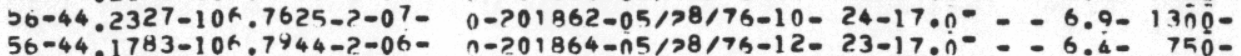

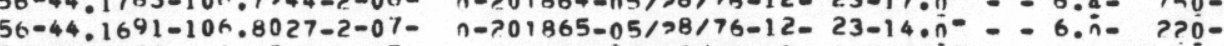
$56-44 \cdot 1877-106.83 n ?-2-07-n=>01866-n 5 / 38 / 7 h-13-21-7 \cdot n^{\circ}-5 \cdot 5-50-$ $56-44.1569-106.9180-2-00-\quad n->01867-n 5 / 28 / 76-15-19-13.0^{-}=-5.7-1800-$ $50-44.1500-106.9697-2-07-00-201869-05 / 38 / 74-15-22-14.00^{-}=5.7-550-$
$4-1-7---1--4-3-2-1--1-1-=$ 6-1-7- - - - - $-4-3-2-1--1-9-$

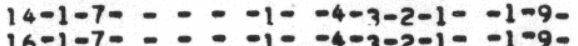
$16-1-70=--1--4-3-2-1--1-90$ $12-1-7-\ldots--1--4-3-2-1--1-9$

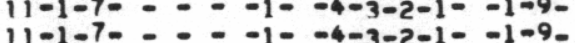

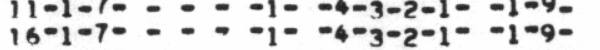
$16-1-7-\ldots-1--4-3-2-1--1-9$ $12-1-7-0-1--4-3-2-1--1-9-$
$19-1-7-=-1--4-3-2-1--1-9-$ $19-1-7-1-0-1--4-3-2-1--1-9-$
$17-1-7-0-1--4-3-2-1--1-9-$ $1-1-7---1--4-3-2-1--1-8-$ $12-1-7-15-1-7-1=-1--4-3-2-1--1-9-$
$13-1--4-3-2-1--1-9-$ $16-1-7-1=-1--4-3-2-1--1-9-$
$1>-1-7-1--1--4-3-2-1--1-9-$ $17-1-7-1-=-1--4-3-2-1--1-9-$
$14-1-7-0-1--4-3-2-1--1-9-$

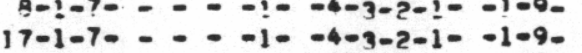
$16-1-7-\ldots--1--4-3-2-1--1-9-$ 17-1-7-- - - - - -4-3-2-1- -1-9$12-1-7---1 \div-4-3-2-1--1=9-$ $12-1-7-1--1--4-3-2-1--1-9-$
$17-1-7-1-1--4-3-2-1--1-9-$

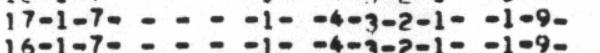
$3-1-6-6-6-2-3-1--2-3-2-1=-1-$ $4-1-7---3-1--2-3-2-2--1-3-$ $5-1-8-4-8-4-4-1-2-2-3-2-2--1-$
$4-1-70--4-1--2-2-2-2--1-3-2 i-$ $4-1-7---4-1--2-2-2-2--1-3-2 i=$
$5-1-7---4-1--2-3-2-1--1-8-$ $5-1-7---4-4-1--2-3-2-1--1-8-$
$5-1-6-5-6-3-4-1-2-2-3-2-1--1-$ $0-1-6-4-6-2-4-1-1-2-3-2-1--1-$ $5-1-7-0-4-1--2-2-2-1--1-4-=80$ 5-1-7- - - -1- -2-2-3-1- -1-4-10-75 $4-1-7-0-0-4-2-1-1--1-4-2-$ $11-1-6-5-6-3-4-2-2-4-3-3-1--1-=$ $15-1-6-5-6--3-2--3-5-3-1--1-0$
$6-1-7--0---1--4-3-2-1--1-80$ $6-1-7---1--1-4-3-2-1--1-8=$ $7-1-4-6-8-2-3-1-1-1-2-4-1--1-$ $6-1-7-4-6-3-3-1-1-1-3-3-1--1--$ $3-1-6-1-7-3-3-1-2-2-3-2-1=-1-3-2 n-$
$4-1-7-0-10-2-3-2-1-1-4-30$ $4-1-7-1-0-1--2-3-2-1--1-4-?$
$6-1-6-0-0$ $16-1-6-1-0-1-4-2-2-1--1-3-100$ $0-1-6-4-6-2-3-1-2-4-3-3-1--1-$
$2-1-7-6-6-2-3-1--1-2-2-1--1-$ $9-1-4-4-4-4-4-1-2-2-3-3-2--1=$ $11-1-4-4-4-4-4-1-2-2-3-4-2--1-$
$3-1-6-4-6-2-3-1-2-2-3-4-2--1-$ $4-1-8-6-8-3-4-1-2-2-3-3-2--1-$ $11-2-7-5-8-4-4-1-2-1-4-5-1--1-=$
0.17

0.17

0.16

0.10

0.15

0.10

0.15

0.45

0.38

0.10

0.18

0.10

0.10

0.10

0.10

0.10

$0.1 \mathrm{c}$

1.89

0.25

2.26

0.63

0.10

2.99

0.10

0.10

0.48

0.5 ?

0.10

0.39

0.40

8.5 ?

0.10

2.68

0.52

1.97

0.74

1.72

1.31

0.46

2. 11

7.97

0.30

0.55

0.10

0.10

0.10

0.23 
LASL Uranium Hydrogeochemical and Stream Sediment Reconnaissance Data Listing

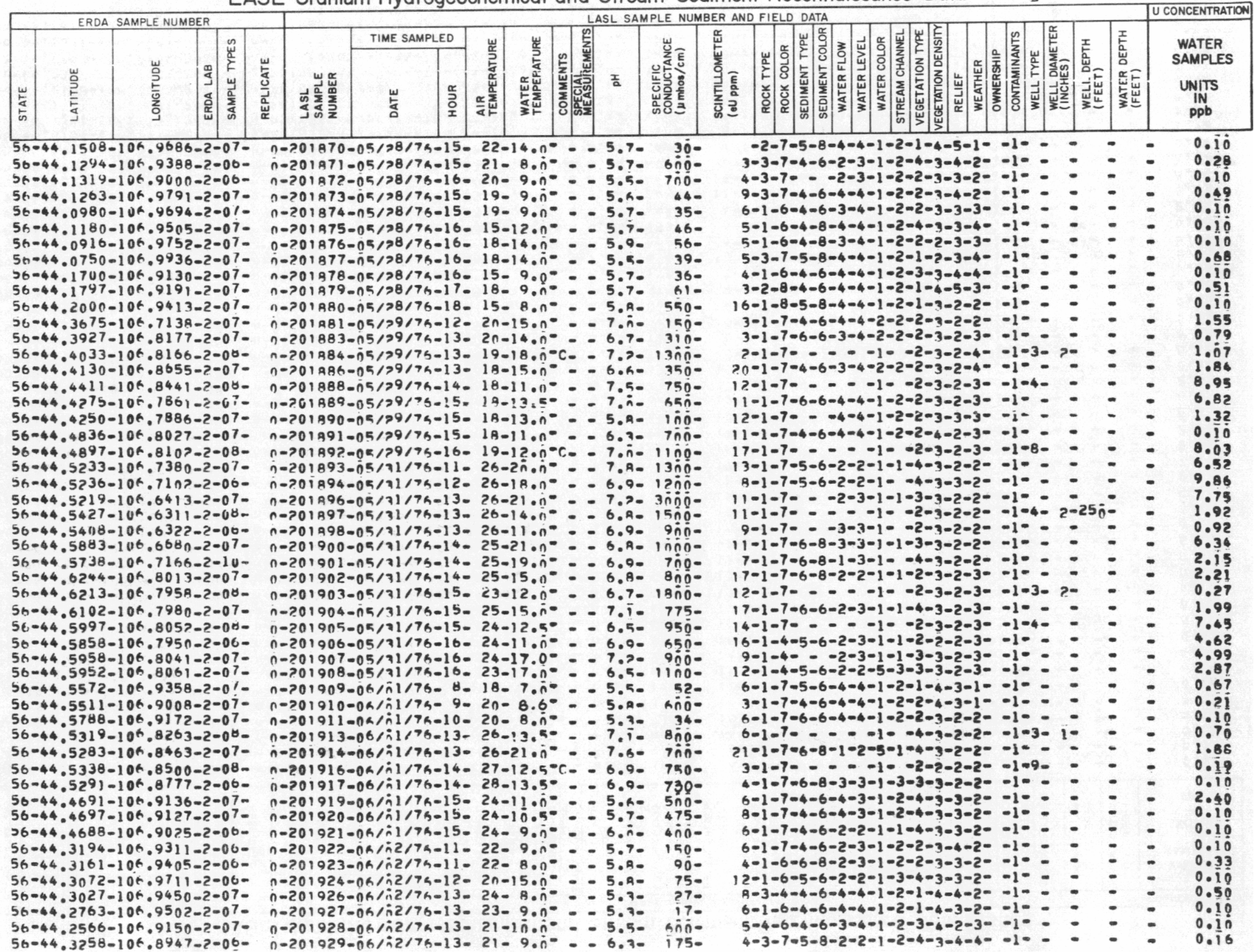


LASL Uranium Hydrogeochemical and Stream Sediment Reconnaissance Data Listing

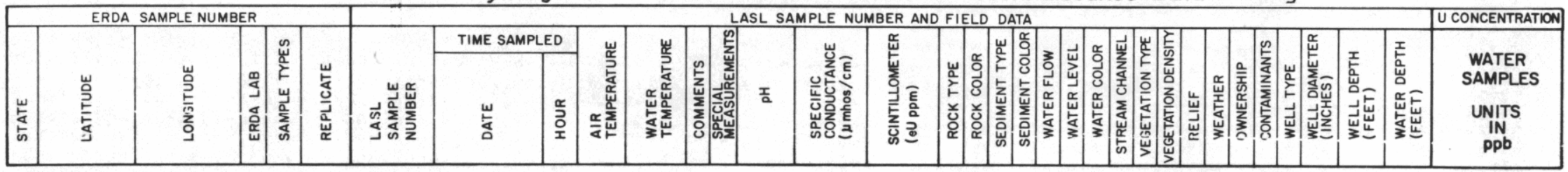

$56-44,3238-106,8338-2-00-$

$n-201930-n a / i 217 a-13-2 n-8 . n^{-}-0.301800-$ $56-44.0230-105.4741-2-08-\quad 0-201931-071>0 / 74-13-23-12.00=-6.2-1600-$ (36-44.0210

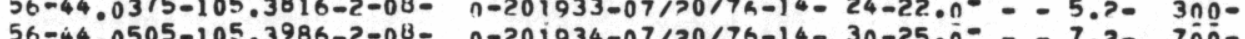
$56-44.0505-105.3986-2-00-n=201934-07720776-14-3 n-25 . n=-7.20$ - $7 n 0-$ $56-44.0738-105.3994-2-10-0-201935-07 / 20 / 74-15-3 n-26.00-0.6-3-2 n 0-$

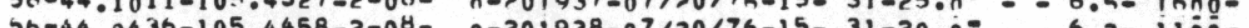
56-44.0436-105.44582-040 n-201938-0740 56-44.0597-105.4283-2-08

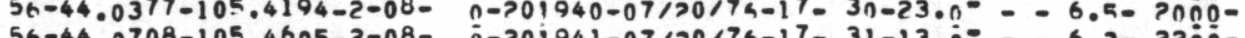
56-44.0708-105.46n5-2-08- n-201941-077>077n-17-31-13.n- - 6.3- 2?nn$56-44.1152-105.4505-2-0800-201943-07170 / 75-17=29-15 . n=-6.7-1$ - 1 nno56-44.0163-109.3094-2-08- n-201944-n713177n-10-22-17.n- - 6.5- 11 - n$56-44.0863-105.2572-2-08-n=>01949-07 / 23776-11-24-17 \cdot n-c-0.7-6 n 0-$

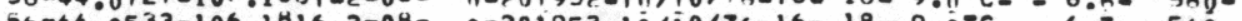
$56-44.0316-106.2019-2-08-$ n-? $01955-10 / 10174-17-17-11.0-2-6.8-550-$

$2-1-7-6-6-2-3-1-2-4-3-4-3--1--$ $3-1-7-\ldots---4-3-2-2--1-1-$

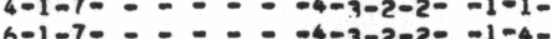
$4-1-70$
$4-1-1-60$ $6-1-6-4-8-2--4-3-2-2--1-=$

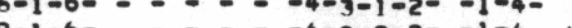

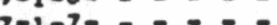
$4-1-6-\ldots-0-4-3-2-2--1-1-6-208-170$ $6-1-6-\ldots-0-4-3-2-2--1-1-0$. $1-1-6-1-2-0-4-3-2-5-1-1-$

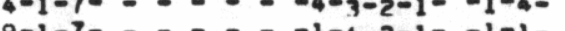
$14-1-7=-10-2-1=-1-4-5-1--1-1-$ $14-1-7=-2=-1-1-3-2-5-2--1-9$.

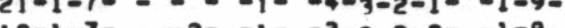


LASL Uranium Hydrogeochemical and Stream Sediment Reconnaissance Data Listing

$56-44.2819-105 \cdot 2041-2-08-$ $36=44.2925-105 \cdot 0997-2-08-$ $3 x-44.3555-105.0591-2-08$ 3h-44. $3555-105.0591-2-08$ $56-44.3466-105.0447-2-01-$ $5 n-44.4106-105.0450-2-10=$ $5 n=44.4186-105.045 n-2-10-$ $36-44.3052-105.0300-2-08-$ $56-44.2491-105.0716-2-08-$ $30-44.1994-105.0888-2-080$ $36-44.725 n-105.6869-2-08-$ $36-44.2819-105.6800-2-09-$ $36-44.2847-105.71119-2-04-$ $56-44.7027-105.4755-2-06-$ $56-4.0555-105.8333-2-07-$ 56-4. $56-44.0244-105.5569-2-10-$ $36-44.6969-105.5180-2-08-$ $56-4.6833-100.6030-2-000$ $56-44.6277-105.6800-2-080$ $56-44.6438-105.6419-2008-$ $56-44.6527-105.6 \times 63-2-09=$ $56-44 \cdot 7413-105.6178-2-08-$ $36-44.9100-105.5738-2-00-$ $56-44.9977-105.5083-2-08-$ $56-44.3316-105.568-2-060$ 36-44.3663-105. $557>-2-000$ $56-44 \cdot 1902-105 \cdot 7227-2-16-$ $56-44.2222-105.7369-2-10-$ $56=44.2097-105.6997-2-07-$ $56-44.1905-105.6469-2-10-$
$56-44.9894-105.0586-2-08-$ $56=44.9847-105 \cdot 3277-2-08-$ $56-44.7800-105.8233-2-07$ $56-44.6811-105.8300-2-06-$ $56-44.9538-105.6630-2-08-$ $56-44.3533-105.6086-2-08$ $56-44.3430-105.4541-2-08$ $56-44.3136-105.6669-2-10$ $56-44 \cdot 3327-105 \cdot 76 \cap A-2-16$ $56-44.3469-105 \cdot 7294-2-06-$ $56-44.2905-105.6338-2-10-$ $56-44.3305-106.6061-2-06$ $56-44.3397-106.7444-2-08$ $56-44.1941-104.6311-2-07$ $56-44 \cdot 2769-10+\cdot 5647-2-10-$ $56-44.0311-105.7177-2-08-$ $56-44.0244-105 \cdot 7238-2-08$ $56=44.1163-105.7086-2-08$ $56=44.1419-105.5313-2-08-$ $n-200001-03 / 27 / 7 a-12-11-9 . n^{-} c_{-}-6.9-22 n \overline{0}-$ $-200015-02 />8 / 76-14-4-7 . n^{-}=-6.6-3 n \pi 00-$ 作 0-2000

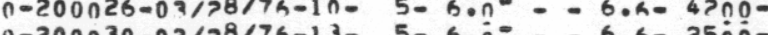
0-200030-0 $0-200032-03 / 3817 h-14-6-6 . n=-6.4-\operatorname{sinno-}$ n-200033-03/>877a-14- $5=6010=0$ $0=200038-03 / 30 / 76-9-7-9.00--6.4-14 n 0-$ n-200n43-03/70176-13- $7-7 . n=-6.704$ nnn-

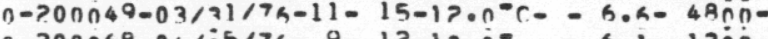

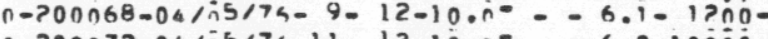
$0 \rightarrow 000 n 72-04 / n 5 / 7 h=11-13-1000^{-}-$- 6.8-1nnnO$n=200100-02 / 30 / 75-14-11-100 n-=7.4-5 n 00-$

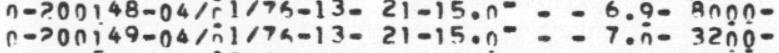
$0 \rightarrow>00 i 64-03 / i 2175-12-4-5 . i^{-}-6.4-33 n 0^{\circ}-$

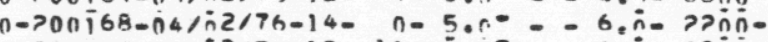
$n=200174-04 / i 3 / 7 a-12-11-1 n \cdot n^{-}-0 . n-29 n 0^{-}$ $n-200184-04 / i 3 / 7 a-10-12-7 \cdot n^{-}--6 . h-44 n 0^{-}$ $n=200187-n 4 / n 3 / 76-11-13-10.0-=-6.5-33 n n-$

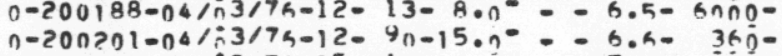

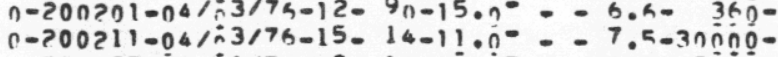
$n-200>23-04 / \pi 6 / 7 h-9-16-10 . n^{-}=-6.0-49 n^{n} 0^{-}$ $n=200>46-04 / 6 / 76-10-14-9 . n^{-}-0.5-3 n n 0-$

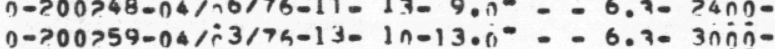

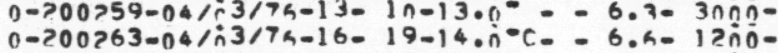
$n=200265-n 4 / 34 / 7 h-9-11-5 \cdot 0_{-C}^{-C}-6 . h-1 n n n \theta-$

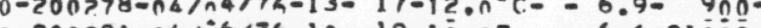
$0-200>91-04 / 26 / 76-16-19-10.00^{\circ}--6 \cdot h-310000-$ $0-200>98-04 / i 6 / 7 a-11-22-13 \cdot n^{\circ} \mathrm{C}-6.9-1 \operatorname{nin} 0-$

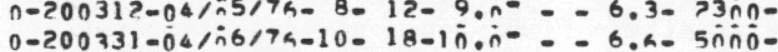
$0-200357-04 / \hat{n} 7 / 76-10-10-9.0^{-C} C_{-}-6.2-3$ Cnño- $^{-}$ $0-200366-14 / 106 / 76-12-\quad-\quad C-$

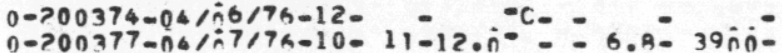
$0-200380-04 / i 7 / 76-12-12-12.0^{\circ}-05.9-470^{-}$ $n-200385-04 / \hat{7} 7 / 75-14-14-13 \cdot n^{*}=-6.9-4790-$

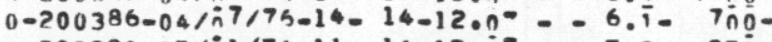
$0=200390-05 / \mathrm{i} 1 / 76-11-16-15 \cdot 0^{\circ}--7.2-950-$ $0=200391-04 / 70776-12-8-14 \cdot 0^{-C C}-7.8-$ ann$n \rightarrow 00395-04 / 70 / 7 h-14-10-14 . n^{-C} C_{-}-6 . h-3 i 50-$ $n-200400-05 / i 1 / 7 a-11-18-16 \cdot n^{-}-0.6-220 n^{-}$

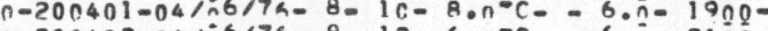
$0=200402-n 4 / i 6 / 7 h=8-12-6 \cdot n=C-6=0 . n-2100-$ $0-700407-04 / 26 / 7 A-10-11-9 . n-C=-6.9-3 \ln n \pi n-$

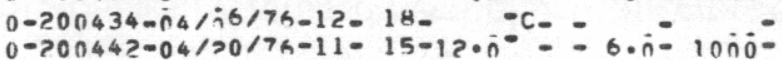

8-1-7- - - - - -4-3-1-2- -1-4- 4 $1 \geq-1-7-4-7-2-3-1--4-3-2-2--1-4-$
$9-1-7-4-7-1-2-5--4-3-2-3--9-$ $6-1-6-4-6-1-3-1--4-3-2-1--1-3=$

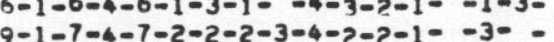
$9-1-7-4-7-2-5-2-3-4-2-2-1=-3-=$ $19-1-6-4-6-1-3-1--4-2-2-3--1-$ $19-1-6-4-6-2-3-1=-4-3-2-3--1-1=$ $9-1-6-4-6-2-3-1--4-3-3-1--1-1-$ $6-1-6-4-6-2-3-1=-3-3-3-2--1-4-$ $8-1-7-4-7-1-2-1=-4-3-2-1--1-$ $13-1-6-4-6-1-2-1--4-3-2-1--10$ $9-1-7-4-7-2-2-3--4-3-2-1--1-=$ $10-1-6-4-6-1-2-2-3-4-2-2-2--1=$ $9-1-6-4-6-1-2-1--4-2-2-3--1-=$ $15-1-6-4-6-2-2-1--4-2-2-3--1-1-$

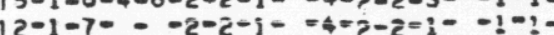
$12-1-6-4-6-2-3-1--3-3-2-1--1-1-6=$ $19-1-7-4-7-3-3-1--4-3-2-1--1-3-$ $15-1-6-4-6-1-3-5-4-4-3-2-1=-1=$ $13-1-7-0-1-5-5=-4-7-2-1--1-1-$ $3-1-6-4-6-2-3-1--1-2-3-2--1-1=$ $10-1-6-4-6-3-3-1--4-7-2-1--1-1=$ $10-1-6-4-6-3-3-1=-4-3-2-1--1-1-$ $12-1-6-4-6-1-2-1=-4-3-3-1--1-=$ $9-1-6-4-6-1-2-1--4-2-3-1--1=$ $15-1=6-4-6-1-2-1=04-2-3-1=-1=$ $12-1-6-4-6-1-3-1--4-3-2-2--1-20$ $9-1-6-4-6---1--4-3-2-2--1-9-$ $16-1-6-4-6-3-3-1--4-3-2-3--1-1-$ $1 n-1-7---2-2-3--4-2-2-2--1-9-$

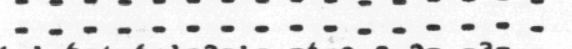
$10-1-6-4-6-1-2-1--4-3-2-2--3-=$ 3-1-6-4-6-1-2-1- -4-3-2-2- -1- $13-1-6-4-6-1-2-1--4-7-2-2--3-=$
$9-1-7-5-8-2-2-1--4-3-3-1--1-=$ $9-1-7-5-8-2-2-1--4-3-3-1--1=-$
$6-1-6--1--1--4-3-1-1--1-4-$

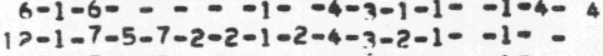
$12-1-7-5-7----4-5-2-1--1-$ $1>-1-6-4-6-3-3-1--4-2-2-3--1-3-$ $9-1-6-4-6-1-3-1--4-2-3-3--1-3-$
$12-1-6-4-6-1-3-3--4-2-2-2--1-3-$ $9-1-7-2-6-2-5-5=-4->-2-1=-1=$
WATER
SAMPLES UNITS Ppb 62.2 32.50 19.6 19.60 28.9 28.90 34.40

51.20

$\begin{array}{r}2.30 \\ \hline\end{array}$

18.10

204.30

33.8

42.50

2.20

19.80

21.6

37.5

44.0

24.0

31.6

18.20

1.20

96.2

67.90

18.7

9.30

0.20

21.60

12.10

29.60
15.30

20.40

34.60

46.20

5.00

29.40

16.60

39.00

25.10

29.50

15.30

15.30

19.20 
LASL Uranium Hydrogeochemical and Stream Sediment Reconnaissance Data Listing

\begin{tabular}{|c|c|c|c|c|c|c|c|c|c|c|c|c|c|c|c|c|c|c|c|c|c|c|c|c|c|c|c|c|c|c|c|c|}
\hline \multirow[b]{3}{*}{ | } & \multicolumn{5}{|c|}{ ERDA SAMPLE NUMBER } & \multicolumn{26}{|c|}{ LASL SAMPLE NUMBER AND FIELD DATA } & \multirow{3}{*}{\begin{tabular}{|c|} 
U CONCENTRATIOW \\
WATER \\
SAMPLES \\
UNITS \\
PPb \\
PPD
\end{tabular}} \\
\hline & & & & w & & & TIME S & & & & & & & & & & & & & & & & & & & & 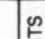 & & $\frac{\tilde{r}}{w}$ & & $I$ & \\
\hline & 㟋 & 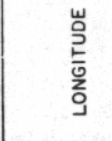 & 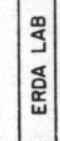 & 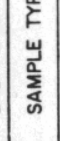 & 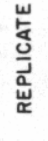 & 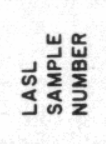 & 岁 & $\begin{array}{l}\text { 올 } \\
\text { 오 }\end{array}$ & 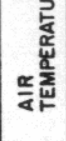 & & 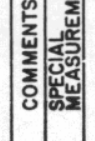 & $\frac{\mathbf{I}}{\mathbf{a}}$ & 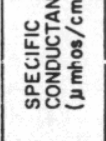 & & & 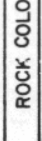 & 点 & 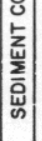 & 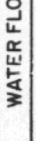 & 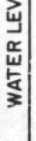 & $\begin{array}{l}0 \\
0 \\
\alpha \\
0 \\
\frac{\alpha}{3} \\
\frac{3}{3}\end{array}$ & 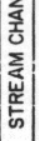 & 至 & 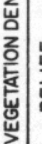 & $\frac{\underline{u}}{\vec{w}}$ & 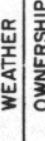 & 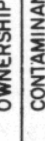 & 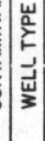 & 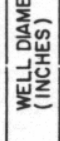 & 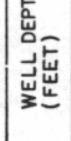 & 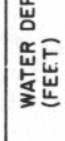 & \\
\hline
\end{tabular}

$56-44.9850-106.3113-2-08-0-200444-04 / 20 / 7 n-12-16-12.0-0=-6.0-400 \bar{n}-$ $56-44.9702-106.3094-2-08-\quad n-200447-04 / 30 / 76-12-18-8 \cdot 0-0-6.9-4000-$ $3644.40750105 .504-2508$ $36=44.6930-106.3408-2-08-$ $36-44.6566-100.2936-2-10-$ $36-44.7416-106.3169-2-08-$ $56-44.7513-106.2744-2-08-$ $56-44.7513-106.2744-2-08-$
$56-44.8283-106.3436-2-16-$ $56-44.8147-106,2673-2-10-$ $36-44.8586-106.0605-2-04-$ $56-44.8586-106.0605-2-08-$
$56-44.1119-106.1863-2-08-$ $56-44.1119-106.1863-2-08-$
$56-44.0669-105.06488-2-08-$ $56-44.4605-105.6813-2-08-$ $56-44.4605-105.6813-2-08-$
$56-44.3847-105.6611-2-08-$ $56-44.3847-105.6611-2-08-$
$56-44.3963-105.6608-2-08-$ $56-44.4069-105.4713-2-06-$ $36-44.74: 13-105.8852-2-02-$ $56-44.6683-106.0783-2-07-$ $56-44.0486-106.0477-2-07-$
$56-44.4427-106.3544-2-06-$ 56-44.3333-106. $2211-2-07-$ $56-44.8797-106.3157-2-08-$ $56-44.9358-106.3627-2-08-$
$56-44.7986-106.4205-2-110-$ $56-44.7986-106.4205-2-10-$
$56-44.8138-106.3833-2-10-$ $56-44.8138-106.3833-2-10-$
$56-44.7872-106.4511-2-10-$ $36-44.9750-106.3986-2-04-$ $56-44.6941-104.4561-2-10-$ $56-44.7152-106.4261-2-10=$
$36=44.6852-106.4719-2-10=$ $56-44.6330-106.4600-2-08-$ $56-44.6488-104.4638-2-10-$ $56-44.5283-10 \% .4636-2-n 8-$ $56-44.5227-106.4461-2-08-$ $56-44.5208-106.4113-2-10-$
$56-44.5886-106.4683-2-10-$ $56-44.5886-106.4683-2-10-$
$56-44.7180-106.5638-2-06-$ $36-44.5786-106.5188-?-10-$ $56-44.7355-106.6341-2-06-$ 56-44.7991-106.7441-2-10$56-44.7869-106.6827-2-10-$ $56=44.7947-106.6155-2-06-$ $36-44.27111-106.7694-2-06-$ $56-44.2766-106.7655-2-07-$ $56-44.2969-106.8050-2-07-$ $56-44.2833-106.7861-2-00-$ $56-44.3288-106.6672-2-0 \%=$
$56-43.6447-106.4677-2-10=$ $56-44.8325$ $56-44.5116-106.2583-2-10$ $0 \rightarrow 200477-04 / 14 / 76-11-17-13 \cdot 0^{-C} \mathrm{C}-$ - 6.R- $27 \mathrm{i}^{\circ}-$ (1) $200484-04714776-12-16-150 n-=6 . R-39 n n-$ $n=>00489-04 / 14 / 7 a-13-20-11.0-0-6.5-3600-$ n-200490-04 $n-200491-04 / i 4 / 76-15-17-15 \cdot n^{\circ}-0.5-3900-$

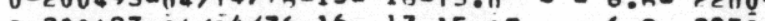

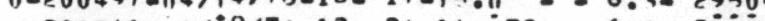

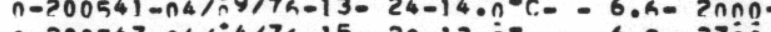
$n-200547-04 / n 4 / 7 h-15-2 n-13 \cdot n^{-}-6.7-37 n 0-$ n-200562-041i0/7h-13- 14-1!.n-c- - 6.2- 35non-200563-04/10176-14- 1n-1non-C- - 6.5- 45no-

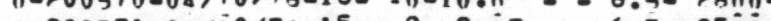
n-2.00571-04/10/7a-15-9-9.0--6.5- 35ño$n-200574-04 / 12776-7=10-9 . n-=6 . n-2800-$ $0-200613-04 / 4776-16-13=9.0--6.4-5 n 000-$ 0-200k20-04/n9/7h-10- 18-12.0- - 6.h- 7nnO$0-200624-04 / 29175-11-23-16 \cdot n--0.6-42 n 0-$ n-200hk1-04/13/7h-12- 22- $0 . n-c=-6 . h-4600-$ $n \rightarrow>00676-n 4 / 14 / 75-10-14-12 . n^{\circ}=-7.5-90100-$

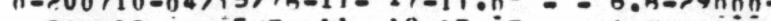
$0-200713-04 / 15 / 7 a-11-18-15 \cdot n^{-}-0^{-}-6.2-40 n 0-$

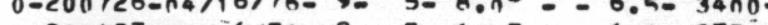
$0-200727-04 / 16 / 7 h-9-5-6 . n^{\circ}-0.4-3750^{-}$ $0-200730-04 / 66776-9-4-6.0-20.7-28 n 0-$ $n->00736-04 / 16 / 7 h-100$ 8-100n- - 6.3-785no$n-200742-04 / 21 / 76-10-12-9.0^{-}-6.3-3 i n 0-$ $0-200745-04 / 31 / 7 h-11-11-12.00=-6 . h-44000$ $0-200746-n 4 / 2177 h-11=13-120 n=-6.9-16 n n 0-$

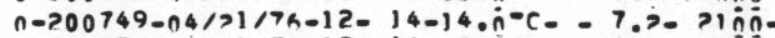

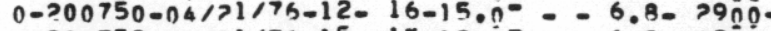
$0-200759-04 / 3177 h-15-15-13 \cdot n-2.3-4300-$

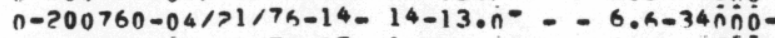
$0-200761-04 / 71 / 75-15-14-11.0^{-}=-6.3-17 n 0-$ $0-200763-04 / 21776-15-15-12.0^{-}=-6.3-1$ - 1 - $00-$

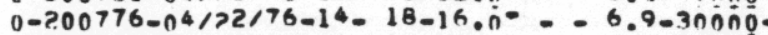
$0-200787-04 / 72 / 76-16-18-15 \cdot 0^{\circ}-0.3-1250^{\circ}$ $0-200789-04 / 24 / 76-10-19-9.0-0.6 .5=11 n 0-$

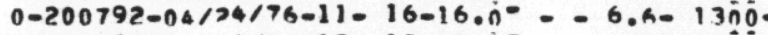
$0 \rightarrow>00793-04 / 74 / 74-12-18-17 \cdot 0^{\circ}-0.3-75 n \hat{n}$ $n=20080 n=04 / 24 / 76-13-20=19 \cdot n^{-}=-6.8=21 n 0$ $n=200812-04 / 33 / 76-11-10-6 . n-C=-6.5-34 n 0-$ $0-200915-04 / 73 / 7 a-15-12-10 \cdot n^{-}-6.4-2150-$ $n=200816-n a / 73 / 76-15-8-10 . n^{-}-0.5-1350$ $0-200918-04 / 33 / 76-16-10-9.00-0.4-1800-$

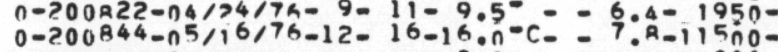
$0-200857-04 / 74 / 76-15-18-2 n_{0} n^{-}-0.9-34 n \pi n 0^{-}$ $0-200865-04 / 75 / 76-12-19-17.0^{-}-0.3-2450-$
$15-1-7---2-2-1--4-2-2-1--1-1$ $\begin{aligned} & 9-1-7---1-3-3-1--4-5-2-1--1-1- \\ & 15-1-7-4-7-1-2-2-04-3-3-2--1-3-\end{aligned}$

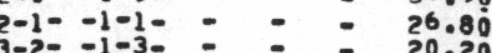
$12-1-7---2-2-1--4-2-2-3--1-3-6-300-=40.90$ $9-1-7-5-7-1-2-2--4-2--2--1--\div \quad 105.70$ $7-1-7--2-3-1--4-2-2-2--1-1-\div-12.70^{\circ}$ $9-1-7--2-3-1--4-2-2-2--1-1-\div=13.30$ $9-1-7-4-7-1-2-2--4-2-2-2--1=$ $9-1-7--2-3-2--4-2-1-2--1-21.30$ $6-1-6-4-6-3-3-1--4-3-1-1--1-9-\quad-\quad 41.00$ $12-1-6-4-6-3-3-1--4-3-2-2--1-3-=2908$

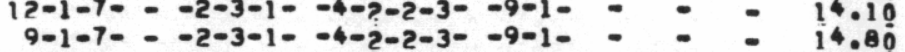
$16-1-7--2-3-1--4-5-2-3--1-1-=-13730$ $12-1-7---2-3-2--4-2-2-3--1-1-\quad-\quad-13.20$ $12-1-7--2-2-1--4-2-2-3--1-\div=-20.80$ $6-1-6-4-6-2-3-1--4-2-3-3--1-1=$ $16-1-6-4-6-1-2-1-2-2-2-2-1--1-\square-75.10$ $12-1-6-4-6-2-2-1--2-2-2-1-3-3-\div-36.10$ $14-1-6-4-6-2-2-1--3-2-4-2--9-\div ;=-13.40$

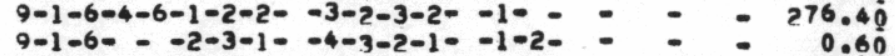
$10-1-60--2-3-1-4-4-3-2-1--1-2-:=0250$

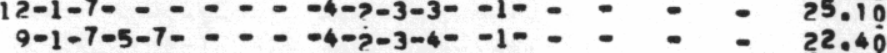
$9-1-7-5-7-\div=-4-2-3-4-1-\div=22.40$ $10-1-7---2-2-1--4-7-2-4--1-1-\quad-\quad 70.90$ $18-1-6-4-6-1-3-1--4-3-2-2--1--\quad-\quad-26.30$

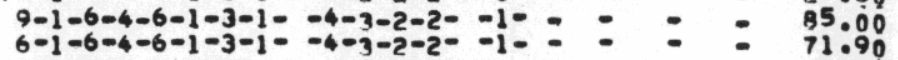
$9-1-6--2-2-3--4-3-2-2--1-9-\quad-\quad 21.60$ $6-1-6-4-6-1-2--4-3-2-2--1-=26.30$ $3-1-6--3-3-1--4-3-2-1--1-2-\quad-\quad 705.90$ $12-1-6--3-2-1=-4-3-2-1--1-2-\div ;-87.60$

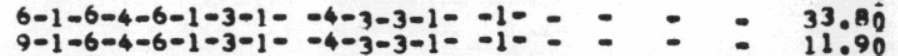
$10-1-7-5-7-2-2-1--4-2-3-2--1--\quad-\quad-20.20$ $4-1-7-4-7-1-2-3--4-2-1-2--1-0-17.78$

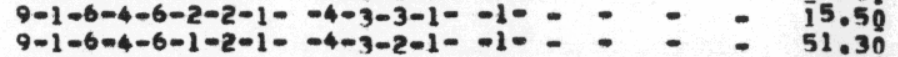
$9-1-6-4-6-1-2-1--4-3-2-1--1-\div ;$
$12-1-6-4-6-1-3-1--4-3-2-1--1-$ 6-1-6-4-6-2-2-1--4-3-3-1--1-- - - $25.80^{\circ}$

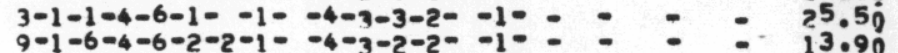
$1>-1-6-4-6-2-3-1--4-3-3-2--1-\quad-\quad-19.30^{\circ}$ $11-1-6-6-8-2-2-1-2-4-3-3-2--1--\quad-\quad 17.20^{\circ}$ $3-1-6-4-6-1-2-1--4-3-1-1--1-5:=$
$6-1-7-5-6-1-3-1--4-3-3-2--95:-17.80$ $9-1-6-4-6-1-3-1--4-3-3-1--1-\div=-$
$6-1-7-5-7-2-2-2--4-2-2-2--1-$ 
LASL Uranium Hydrogeochemical and Stream Sediment Reconnaissance Data Listing

占

\begin{tabular}{|c|c|c|c|c|c|c|c|c|c|c|c|c|c|c|c|c|c|c|c|c|c|c|c|c|c|c|c|c|}
\hline \multirow[b]{3}{*}{ 岁 } & \multirow{3}{*}{ 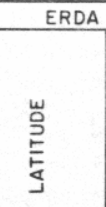 } & \multirow[b]{3}{*}{ 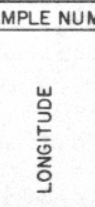 } & \multirow{3}{*}{\multicolumn{2}{|c|}{ 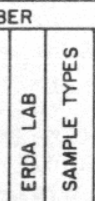 }} & \multirow[b]{3}{*}{ 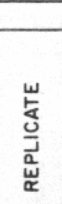 } & \multirow[b]{3}{*}{ 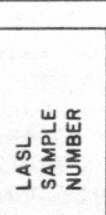 } & \multirow{2}{*}{\multicolumn{2}{|c|}{ TIME SAMPLED }} & \multirow{3}{*}{ 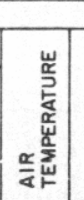 } & \multirow{3}{*}{ 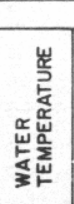 } & \multirow{3}{*}{ 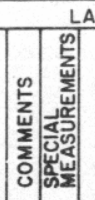 } & \multirow[b]{3}{*}{ I } & \multirow[b]{3}{*}{ 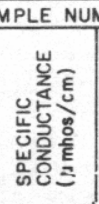 } & \multirow{3}{*}{ 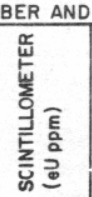 } & \multirow{3}{*}{ 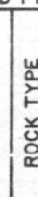 } & \multirow{3}{*}{\begin{tabular}{l|l|l} 
& \\
& & \\
0 \\
0
\end{tabular}} & DATA & & & & & & & & & & & Ucor \\
\hline & & & & & & & & & & & & & & & & & \multirow{2}{*}{ 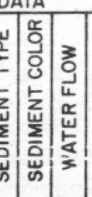 } & \multirow{2}{*}{\multicolumn{2}{|c|}{ 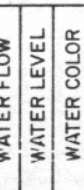 }} & \multirow{2}{*}{ 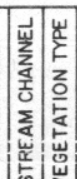 } & \multirow{2}{*}{ 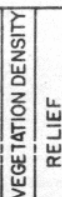 } & \multirow[b]{2}{*}{ 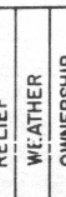 } & \multirow{2}{*}{\multicolumn{2}{|c|}{ 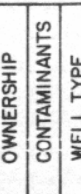 }} & & \multirow[b]{2}{*}{ 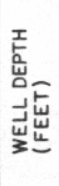 } & \multirow{2}{*}{ 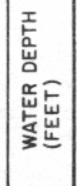 } & \multirow[b]{2}{*}{$\begin{array}{c}\text { WATER } \\
\text { SAMPLES } \\
\text { UNITS } \\
\text { IN } \\
\text { Ppb }\end{array}$} \\
\hline & & & & & & & 造 & 홈 & & & & & & & & & & & 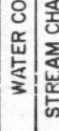 & & & & & & & & & \\
\hline
\end{tabular}

$56-44.5033-106.2702-2-1 C-0-200866-n 4 / 25 / 7 h-13-18-16 \cdot 0-C-0-6.9-430 \overline{0}-$ $36-44.3491-106.5222-2-08-\quad n-200989-05 / i 1 / 7 h-16-2 n-17 \cdot n-C=-6 . n-1 A n \hat{n}-$ $56-44.7611-106.8050-2-08-0-200891-04 / 29 / 74-10-12-9 \cdot 0-r-6.9-16 n 0=$

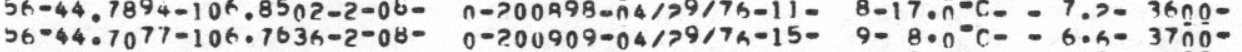
56-44.8752-106.8630-2-08- n-200914-n4/28/7a-10-12-10.n- - - 6.i- $1400-$ $36-44.9830-106.8122-2-08-0-200921-04 / 30 / 7 h-13-18-11 . n-=-6.7-35 n 0-$ $56-44.9838-106.7194-2-08-0-200925-04 / 30 / 79-14-20-12.0-:-6.9-160100-$ $56-44.9627-106.7200-2-08$ - $0-200926-10 / 30 / 76-14015-14.0-0-6.5-47 n 0-$ $56-44.9383-106.6294-2-08-0-200933-04 / 30 / 7 a-16-14-130 n-c=-6.2-45 n 0^{-}$ $56-44.9133-106.6338-2-10-0-200934-04 / 70 / 74-14-17-14.0-0.8 .1-71000-$ $36-44.918 n-106.6166-2-06-0-200935-06 / 90 / 79-14-17-16 . n-=-6.4-41 n 0-$

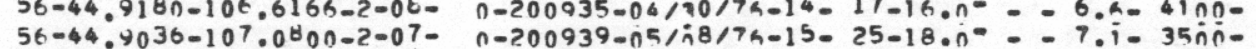

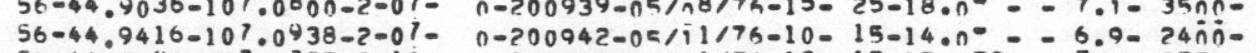
$56-44.9694-107.0325-2-10-0-200944-05 / 11 / 75-12-15-15 \cdot 0-C-$ - 7.7- $7550-$

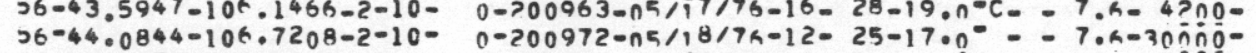

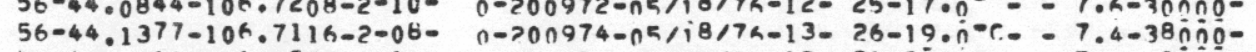

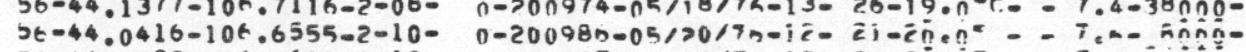

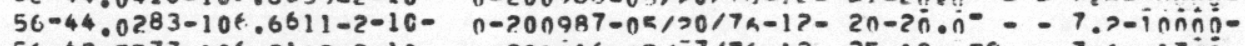

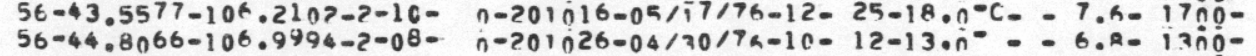
$56-44.8066-106.9994-2-08-$
$56-44.7555-106.9683-2-10-$
$5-701030-06 / 30 / 75-14-11-14.0-:-0.8-13 n 0-$

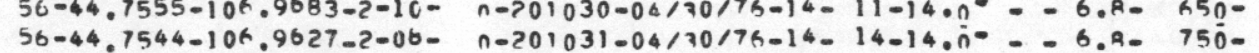
$56-44.7505-104.9750-2-10-0-201032-04 / 30 / 74-15-13-1500^{-}$- - G.A- 550$56-44.7527-10 \mathrm{~h} .9480-2-08-n-201037-05 / \hat{1} 1 / 76-11-13-11.0=-6.8-1950-$

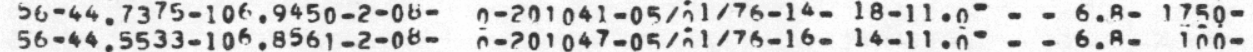

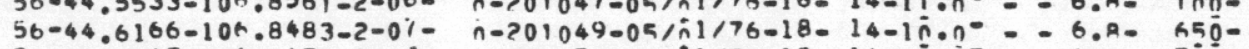

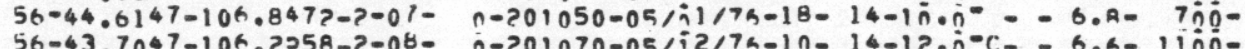

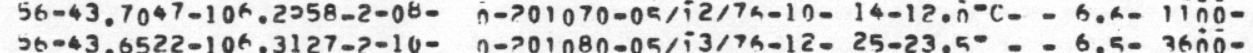
$36-43.6522-106.3127-2-10-\quad n-701080-05 / 33 / 7 h-12-25-23.5-:-6.5-36 n 0-$
$56-43.8613-106.6544-7-06-0$

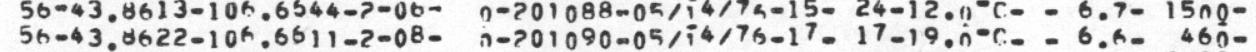
J6-43.9016-106.6572-2-08- n-P01091-05/i7/7G-11- 24-10.5-C- - 6.9- 14 ño-

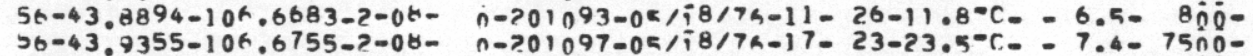
$56-43.9716-106.5022-2-08-$ n-201106-05/i9/7h-15-23-11.5-c- - 6.h- 17ñn$36-44.9194-106.9144-2-1 n-0-201132-02 / 77 / 74-16-20-18 \cdot 0=-2=7.5-4 n n 0-$

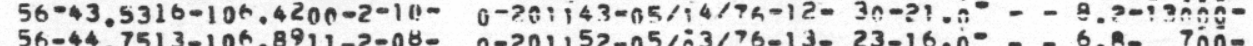

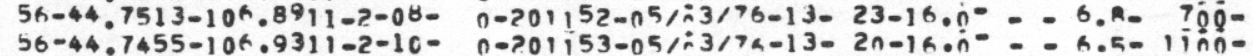
$56-43.5150-10 \mathrm{~N} .7086-2-07-0.9-31500-$ 56-43.5361-106.6800-2-10- n-201195-n5/Aं7/76-19-17-14.0. - - 6.A-245ñ-

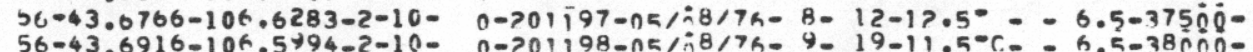
b6-43.6344-10K.6019-2-07- n-701200-05/i8/76-11- 19-19.0 - - 6.8-33riñ$56-44.2750-106.3194-2-1 n-0-201222-05 / 31 / 76-15-3 n-25 . n-0-6.3-2 n n 0=$

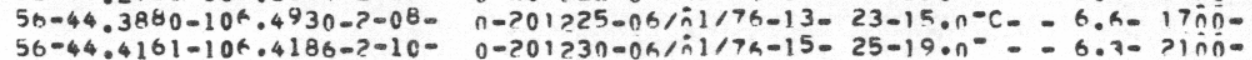
56-44.8072-107.0933-2-07- i-201303-05/13/74-14-26-12.n- - 7.3. 9ก́-
$16-1-7-5-7-2-2-2--4-2-2-2--1--0$ $11-1-7--3-3-1--4-2-2-1--1--30-300-$ $23-1-7-=-3-3-1=-4-2-3-2--5-8=$

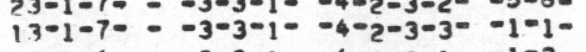
$6-1-6--3-3-1--4-3-2-1--1-3-$ $6-1-6---3-3-1=-4-3-3-2--1-3-$ $9-1-60--3-3-1=-4-3-3-2=-1-3-$ $9-1-6-2-6-3-1--4-3-3-1-1-1-9-$ $6-1-60--3-3-1--4-3-2-1--1-3-$ $12-1-4-6-8-2-3-1-2-4-3-2-1=-1-$ $6-1-7-5-6-3-3-3--4-3-2-3--1-$ $6-1-7-5-6-3-3-3--4-3-2-3--10$
$15-1-7-5-6-1-3-1--4-3-4-4--9-$ $9-1-7-5-7-2-3-1--4-7-2-1=-1=-$ $6-1-7-5-6-1-3-1--4-3-1-1--1=$ $3-1-7---2-3-1--4-7-1-1--1-9$ $6-1-7-5-6-1-3-3--4-3-2-5--10$ $9-1-7-5-7-1-2-2--4-7-3-1--10$

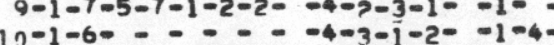
$10-1-6-6-8-2-2-1--4-3-2-2--1-$ $10-1-60-\cdots--4-3-2-2--1-4-$ $11-1-6-4-8-3-2-1=-4-3-2-2--1-$ $5-1-6-0-2-0-4-3-1-1--1-4-$ $16-1-6-0 .--4-3-2-1--1-4-$ $9-1-40---0-4-3-5-1--1-40$ $12-1-6-4-6-2-2-1--4-3-2-1--1=$

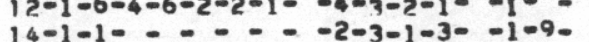
$12-1-1-5-7--2-4--4-5-3-1--1=-$ $11-1-1-\ldots-0-3-2-2--1-4-6-155-60$ $15-1-1-1-2-0 .-3-5-1--1-1-$

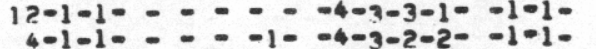
$1-1-7-0-0-04-3-2-1--1-9-3-$ $1-1-7-5-7-1-3-1--4-4-2-1--1=-3$

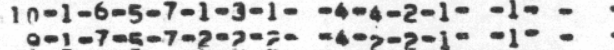
$9-1-6-0-0-4-3-2-2--1-4-$ $19-1-8-6-8-2-2-1=-4-7-2-2=-1=$ $12-1-7-4-7-3-3-1-2-4-2-3-1=-1=-$
$9-1-7-5-6-\cdots-1-4-7-1-1=-1=$ $9-1-7-5-6-2--1--4-3-3-3--1=$ $11-1-6-5-6-1-2-1--4-3-3-1--1=$ $11-1-7-4-6-2-3-1-2-4-3-3-1=-1=-$ $10-1-6-5-6-1-2-2--4-2-3-1-1-1-$
$6-1-6-1-1-2--3-3-3-1--1-3-$ $6-1-6-1$
$3-1-6-:-2:-3-3-3-1--1-3-$ $6-1-1--2-3-1--4-4-1-1--1-$
58.50

19.7 19.70 191.90 91.90
46.20 23.10 9.80 4.80 14.20 $51 \cdot 10$
295.80 34.60 39.70 73.90 23.80 16.60 16.60
84.70 84.70 71.50 32.50 42.20 19.30 19.30 16.40 15.00 52.00 17.50 15.10 15.80 15.30
17.90 12.50 38.30 39.20 39.20
72.80 72.80 66.0 13.00 18.00 45.4 15.70 14.9 24.40
10.30 14.10 30.10 20.40 19 21.60
37.50 
LASL Uranium Hydrogeochemical and Stream Sediment Reconnaissance Data Listing

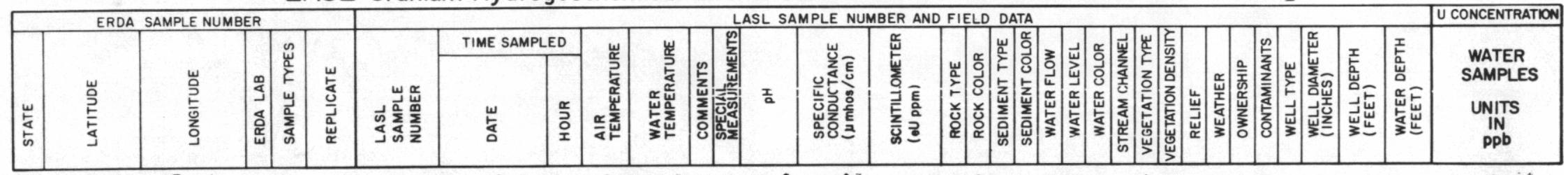

$56-44.7833-107.0411-2-10-0-201306-05 / 13 / 7 a-16-28-24 \cdot n-0-8.00-35 \hat{0} 0-$ $36-43.5541-106.8041-2-06-$ 56-43.5527-104. $7930-2-06-$ $50-43.5472-106.8041-2-06-$ $36=43.6541-104.8708-2-08-$ $56-43.7055-106.8194-2-08$ $66-43.6597-106.8458-2-06=$ $56=4309708-106.7847-2-10=$ $36-430944-106.7541-2-10-$ $36-43.9288-106.7133-2-10=$ $56-43.5638-106.8666-2-08=$ $56-43.8222-106.8555-2-08-$ $50-43.7972-106.8527-2=08$ $56-43.7861-100.8375-2-06$ $56-44.95770105 .1586-2-00$ $56-44.7538-10 \leq .2172-2-08-$ $56-44.6744-105.1263-2-08$ $56-44.3758-105.8008-2-08-$ $56-44,1138-105.8666-2-06-$ $36=44.0694-105.7577-2-06-$ $56-44.0327-105.7811-2-06-$ $56-44.0922-106.0255-2-08-$ $56-44.2155-104.092 ?-2-08-$
$56-44.7297-107.067 ?-2-07-$ $56-44.7297-107.0672-2-07-$
$36=44.2038-106.7522-2-10-$ $50-44.1597-106 \cdot 7675-2-07-$ $56-44.3861-106.7572-2-07-$
$50-44.4383-106.8522-2-0 \%$ $56-44.5786-106.6394-2-00-$ $56-44.5313-106.8466-2-0 \%$ $56-44.0366-106.1763-2-08-$ $56-44.0172-106.1005-2-08-$ $5 h-44 \cdot 1666-106 \cdot 2083-2-08-$ $56-44.0825-106.3583-2-08-$ $36-44.0927-106.3072-2-08-$ $56-44,0833-106.2705-2-08-$ $56-44.0716-106.2608-7-06-$ $56-44.0658-106.2686-2-06-$
$50-44.0350-106.3197-2-08-$ $n-201483-07 / i 5 / 7 n-14-33-23.0-1-2-7.4-550-$ $n-201490-07 / 55 / 7 n-16-35-2000-c=-700-8000-$

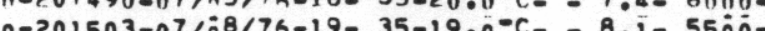

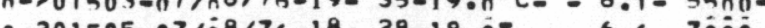

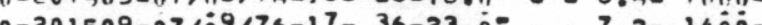
$0-201509-07 / 29 / 7 h-17-36-23 \cdot n-2-7.2-14 n 0-$ $0-201511-0714917 h-18-36-1900-6.8-18 n 0-$ $0-501912-0$ 7

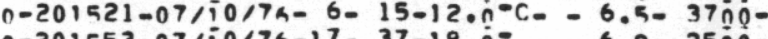

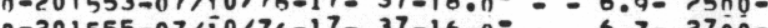
g-?

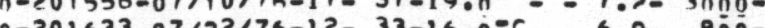
$n \rightarrow 201672-n>1 / 22 / 7 a-17-3 n-14 \cdot n-C-=7.4-$ año(1)-201689-07/=3/76-14-33-13.5- - - i.n- $55 \overline{0}=$ $n-201703-10 / n 8 / 7 a-13-21-11 \cdot n^{-}-0.9-2 n n 0-$ n-201726-1n/ri9/7a- 8- 12-12.ñ - - 6.9- $55 n 0-$ $n=201730-1 n / 89 / 76-9-14-12 . n^{-}-6.9-53 n 0-$ $n-201732-1 n / n 9 / 7 h-9-14-11 \cdot n--6.9-5 n n 0-$ $n-201796-1 \hat{n} / 10 / 7 a-12-20-10 . \hat{n}-r_{-}=6.9-850-$ $n=>0183^{9}-05 / 26 / 7 h-15-21-14 . n^{\circ}-1.4-975-$ $0-201859-05 / 28 / 76-10-23-19 \cdot 0^{-}-0.5-1750-$ $0=201863-05 / 38 / 74-11-27-20.0-2-7.2-33 n 00-$ n作 $n=201915-06 / i 1 / 70-13-27-17.0-:-6020165-$ $n \rightarrow 01957-10 / 10176-18-18-100-0-6.3-165-$ $n=201958-1 n / 10 / 76-18-18-12 \cdot n-C_{-}=6.8-45 n-$ $n=201960-1 n / 11 / 7 k-9-11-1 n \cdot n-C-6.4-8 n 0-$ n-?.01964-1n/17th-10- 14-1non- - 6.8- $1900-$ $n-201967-1 n / 11 / 7 a-10-14-11 \cdot n^{-}=-6.9-2400-$ $n->01969-1 n / 11756-11-16-10.0^{\circ}=-6.9-3 n n 0-$ $0-201971-1 n / 1176-11-16-11.0--6.9-3100-$

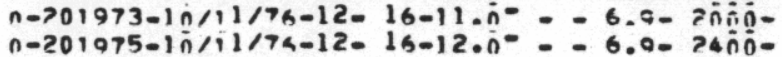

$19-1-6--1-3-2--4-2-2-2--1=-$ $6-1-7-4-6-3-3-1--4-3-3-1--1-=$ $6-1-7-4-6-3-3-1--4-3-3-1=-1=-$ $11-1-7---3-3-2--4-3-3-1--1-7=$

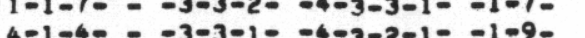
$4-1-4--3-3-1-4-4-3-2-1--1-9-$ $9-1-7-5-6-3-3-1=-4-3-2-1--1=$ $1-1-7-5-6-3-3-1--4-3-2-1=-10-$ $9-1-7-5-6-3-3-1-4-4-2-1--1=$ $11-1-7-5-6-3-3-1=-4-3-2-1--1-$

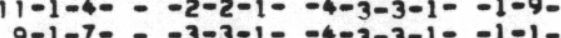
$9-1-7=-3-3-1--4-3-3-1=-1-1-$ $9-1-7=0-3-3-1=-4-3-4-1=-1-1=$ $6-1-7=-3-3-1=-4-3-3-1=-1-9-3-$ $6-1-70=-3-3-1=-4-3-4-1=-9-30$ $8-1-7=-2-1=-4-3-2-1--1=9$ $17-1-7-0-0-1--4-3-7-1--1-8-$ $8-1-7-5-8-3-3-1--4-3-2-1--10-$ $9-1-7-5-6-3-3-1-3-4-3-2-1--1-=$ $14-1-7-5-0-1-04-3-2-1--1-9-$ $14-1-7-1-7-1--4-3-2-1--1-9-$ $12-1-7--5-1--4-3-2-1=-1-9-$
$4-1-6-4-6-3-3-1-2-2-3-3-1=-1-$ $4-1-6-4-6-3-3-1-2-2-3-3-1--1=-$
$7-1-6-5-6--3-1--6-3-2-1--1=-$ $14-1-6-4-6-2-2-1-3-3-3-3-1--1-=$ $15-1-7-6-6-4-4-1-2-2-3-2-2-1-1$
$12-1-7-5-6-3-3-1-2-2-3-2-4-$ $12-1-7---1--1--2-3-2-2--1-4-$
$75-1-7-6-6-3-4-1-2-2-3-2-2-1-$ 21-1-7-- - - - 1- -4-3-2-1- - - $-9-$ $17-1-7--2=-1--3-2-3-2=-1-90$
$16-1-7-0-0-1--4-3-2-1=-1-9$ $16-1-7-0-1-4-4-3-2-1--1-9-$ $16-1-7-1=--1--4-3-2-1--1-1-$ $16-1-7-0-1--4-3-2-1--1-1-$
$17-1-70-1-4-3-2-1--1-1-$ $17-1-7-=-1--4-3-2-1--1-1-$ $16-1-7-\ldots=-1--4-3-2-1--1-1-$
$16-1-70=-1--4-3-2-1--1-1-$
24.10

19.30

20.10

18.10

0.90

23.50

1.60

14.40

16.40

9.40

40.90

41.79

22.70

1.40

7.40

51.10

52.50

59.00

51.70

0.80

1.10

15.50

25.40

59.70

38.10

27.90

16.10

1.50

1.10

1.60

41.90

53.50

63.20

59.00

58.70

59.30 


\section{APPENDIX B}

LISTINGS OF FIELD DATA AND URANIUM CONCENTRATIONS

FOR

SEDIMENT SAMPLES

(Pages 50 through 68) 
LASL Uranium Hydrogeochemical and Stream Sediment Reconnaissance Data Listing

5h-44.3104-105.2327-2-14- $1-200003-03 / 27 / 79-14-9-9.0-C-0-5.2-80=$ $30-44.3644-105.2205-2-15-$ $56-44.4135-105.2277-2-15-$ $36-44 .+405-105.2261-2-14-$ $36=44.3430-105.1123-2-140$ $56=44.3911-105 \cdot 102 ?-2-14-$ $26-44.4588-105 \cdot 0051-2-96-$ $26-44,3466-105 \cdot 0447-2-12-$ $56-44.389+4-105.0561-2-14=$ $36-44,3871-105.0061-2-14-$ $56-44.3986-105 \cdot 0105-2-14=$ $56=44.4364-104.0088-2-14-$ $30-44.41$ dh $-105.0450-2-14-$ $56-44.2644-105.9769-2-90-$ $56-44.2485-105.0705-2-97-$
$56-44.2300-105.01194-2-113-$ $56-44 \cdot 230 n-105.1194-2-13-$ $36-44,2322-105,2136-2-13-$ $56-44.1281)-10=.7016-2-13-$ $56-44.2902-105 \cdot 1516-2-14-$ $36-44,3472-105 \cdot 3461-2-14=$ $56-44,3667-105,3494-2-14=$ $56-44,4411-10=2766-2-14=$ $36-44.4311-105.2691-?-15-$ $56-44,2817-105.6800-2-13-$ $56-44.2733-105.6927-2-13-$ $56-44,2944-105,7263-?-11-$ $36=44.2847-105.7119-2-13-$ $56-44 \cdot 7330-105 \cdot 3233-2-14-$ $56-44,1325-105.2894-?-15-$ $36-44,143)-105.3130-2-14-$ $56=44.0858-105.3563-2-14-$
$36=44.0522-105.345 n-2-15-$ $56-44.084 /-10^{5} .4241-?-14-$ $36-44,7144-105.4580-2-97$ $56-44.1191-105.4761-2-11-$
$56-44.1210-105.4872-2-15=$ $36-44.6911-105.4727-7-11-$ $56-44.7 n 27-105.4755-2-11-$ $36-44.6894-10^{5} .4150-2-15$ $36-44.7083-105.4661-2-96$ $56-44.7100-105.4316-2-97$ $36-44,7261-105.4155-2-97$ $56-44,81127-105.4416-2-14-$ $56-44.797 b-105.4197-2-15-$ $56-44 \cdot 7769-105 \cdot 2805-2-12=$ $56-44.3321-105.3852-2-15$ $36-44,8405-105.3655-2-14$ $36-44,8230-105.2808-?-14=$ $36-44.6747-105.6889-2-13=$ $56-44.756 y-105.6761-2-71=$
$0-200005-03 / 27 / 74-15-10-\quad--$

$n=200013-03 / 28 / 75-11-5-$
$0-200014-03 / 28 / 75-12-5-11.0-C_{-}=6.5-3000=$ $0-700017-03 / 28 / 74-14-5-R_{00-0}-5.7-1800$ $-200018-03,78,75-15-5-9.0-=6.3-1300$ $-? 00023-03 / 28 / 7 h=17-$ $-200 n 26-03 />8 / 7 k-10-5-6 . n-0-6 . k-4200$

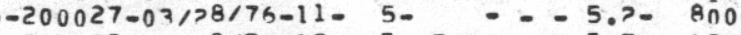
$-200029-03 / 38 / 74-12-5-5.10--5.7-120-$ $-2011330-037>8 / 7 n-13-5=6 . n-0-5 . h=2500-$ $-200031=03 / 78 / 7 n=14=506.0-0.5 .5-360-$ $n=>0 n n 32-03 / 28 / 74-14-6-6.1-=-6.6-70000$ $1=>00036-03 / 70 / 75-8-7-\quad--$

$-200039-03 / 30 / 75-9-7-\quad-C=-$ $-201104 ?-03 / 30 / 74-12-1-7 . n--5.5-130-$ $n \rightarrow>0 n n 46-03 / 30 / 76-13-8-8 . n-=-5.5-90-$ $1-200048-03 / 31 / 74-10-13-9.00-6.9-18000$ - $>00052-03 / 37 / 76-19-9-9.0--5.9-7850-$

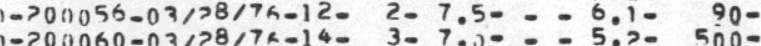
$1=200060-03 / 78 / 7 k-14-3-7.1)=-50.3-500-$ $1=700062-03 / 28 / 7 h-17-3-6 . n=-5.2-140$

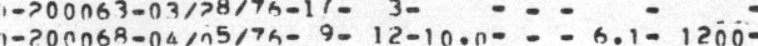
$1=20$ กn $68-04 / n 5 / 7 h-9-12-10 \cdot n=-6.1-12000$

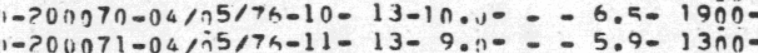
$->00072-04 / 75 / 76=11-13-10.0=-0.8-100000$ $\rightarrow 00082-03 / 29 / 74-13-8-11 \cdot n=-8, n-1200$ $\rightarrow>00083-03 />9 / 76-14-12-$

$\rightarrow>00084-n 3 / 29 / 74-14-1 n-8_{0} n-C_{-}-7.0-500-$ $\rightarrow>00085-03 />9 / 7 k=15-8-R_{0} n-C=-7.4-100=$ - $200087-03 />9 / 75-16-9-2=-0$ $\rightarrow 0009 ?-03 / 30 / 79-10-9-8.11--7.8-100-$

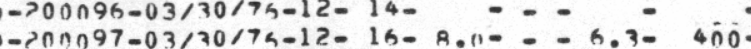

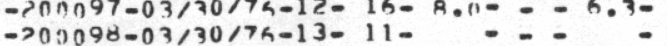
$1 \rightarrow>00099-03 / 70 / 7 h-13-12-8.0--6.1-800-$ $1 \rightarrow 200100-02 / 30 / 79-14-11-1 n \cdot n--7.4-5000-$ $0 \rightarrow 00101-03 / 30 / 76-15-15-$ $0-200102-02 / 30 / 74-15-15-$ $0-200103-02 / 20 / 7 n-15-15-$

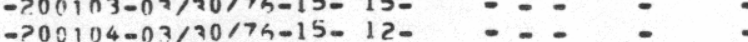
$-200108-03 / 30 / 75-17-9-11 . n--6.3-10000-$ 6- $000109-03 / 30 / 76-17-9-\quad--9_{-}^{-} 0-$ $11-200112-03 / 31 / 76-8-13-6.5=-6.9-800-$ $->00119-03 / 21 / 76-11-24-\quad-D_{-}-$ $0-200122-03 / 31 / 7 k-13-20-14 \cdot n-C-$ - 6.3- 8 noj$1-200125-13 / 31 / 74-13-14-16 . n=-0 . n-17 n 0-$

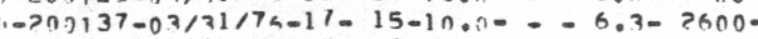
$=200138-04 / 41 / 75-9-18-\ldots$
$6-1-7-5-6-1-3-3--4-3-2-2--9-$
$6-1-7-4-7-0$

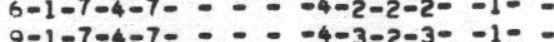
$6-1-7-4-7-1-1-4-3-2-3--1-$
$9-1-7-4-7-1-3-2--4-3-2-3--9-$ $9-1-7-4-7-1-3-2-4-3-2-3-$
$6-1-6-4-6-1-2-2--4-3-2-2--1-$ $6-1-6-4-6-1-2-2--4-3-2-2--1=$ $6-1-6-4-6-1-2-2--4-3-2-2-1-1-$
$3-1-7-4-7---1--4-3-3-2--1-$ $9-1-7-4-7-2-2-2-3-4-2-2-1--3-$ $12-1=6-4-6-1-3-1=-4-3-3-2=-1-$ $9-1-6-4-6-1-3-2--4-2-3-3--1=$ $16-1-6-4-6-1-2-2--4-2-2-3--1-$ $19-1-6-4-6-1-3-1--4-7-2-3--1-$ $19-1-6-4-6-1-3-1--4-7-2-3--1=$ $16-1-6-4-6--\cdots--4-2-3-1--1=$ $9-1-7-5-8-1-2-4-4-3-3-1=-9-$
$9-1-6-4-6-1-2-4--4-3-2-2--1-$ $9-1-7-4-7-1-2-2--4-3-2-1--1-$ $6-1-6-4-6-1-3-2--4-4-2-1--3-$ $9-1-7-4-7-2-3-1--4-3-1-3--1=$

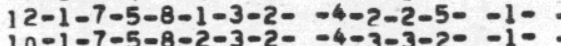
$7-1-7-5-6-2-3-6--4-3-3-2--1-$ $7-1-7-3-8-1--4-3-2-2--1-$ $8-1-7-4-7-1-2-1--4-3-2-1--1-$ $10-1-6-4-6-1-2-1--4-3-2-1--1=$ $13-1-6-4-6-1-2-1--4-3-2-1--1-$ $1-1-7-5-7-1-2-2--4-2-2-2--1-$ $9-1-6-4-7----4-2-2-2--1-$ $12-1-7-5-6-1-2-2--4-2-1-2--1-=$ $9-1-7-4-7-2-2-2--4-3-2-2--1=-$
$18-1-7-4-7-2--7-4-3-2-2-2=1-=$ $9-1-7-4-7-1-2-2--4-3-2-2--1-=$ $12-1-7-4-7----3-3-4-2--1-$ $8-1-7-4-7-2-2-3--3-3-3-2--1=$
$9-1-7-4-7--7--3-7-3-1--1=$ $13-1-7-5-6-2-2-1=-1-3-4-1=-1=$ $9-1-7-4-7-2-2-3--4-3-2-1--1=$ $9-1-7-4-7-\div-4-3-3-1=-1-$
$9-1-7-4-7-0-4-3-2-1--1-$ $9-1-7-4-7-\div-1-4-3-2-1=-1-$
$2-1-7-4-7-0-3-3-2-1=-1-$

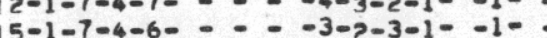
$12-1-7-4-7-1-2-1--4-3-2-1--1-$ $12-1-7-4-7-\cdots--4-3-2-1--1-$ $6-1-7-7-7-2-2-2--4-2-2-1--1=$
$9-1-7-4-7-7-7=-4-3-2-1=-1=$ $9-1-7-4-7-\ldots--4-3-2-1--1$ $9-1-7-4-7-1-2-3-4-4-3-1-1--1=$
$9-1-7-5-5-1-2-2--4-2-2-1=-1=$ $9-1-7-5-5-1-2-2--4-2-2-1=-1=$ $9-1-7-4-7-1-3-2--4-3-3-1-1--1-$
2.90

3.40

3.50

3.70

4.20

3.80

3.10
.78

2.70

3.30

3.10

3.30

3.40

4.70

2.90

3.00

3.90

3.50
3.10

3.30

3.40

3.70

4.20

3.80

3.50

3.90

3.50

5.10

3.00

3.80

2.90

3.70

4.80

3.20

3.50

2.90

3.90

2.80

4.40

3.40

3.20

3.20

4.00

2.90

3.00

3.00

3.20

2.80 
LASL Uranium Hydrogeochemical and Stream Sediment Reconnaissance Data Listing

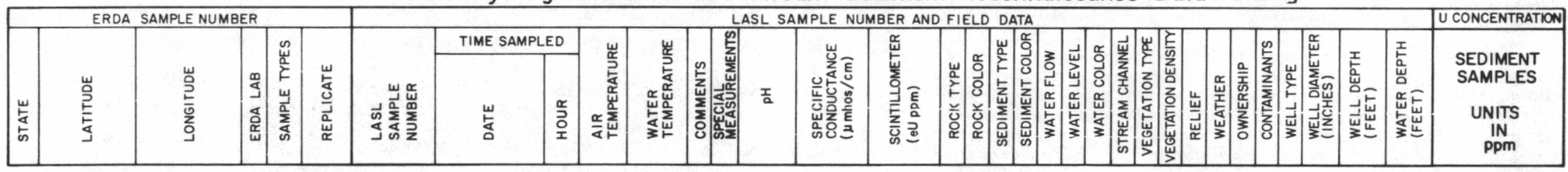

$56-44.7855-105.6761-2-14-$ $36-44.031$ b-1 $05.8105-2-96-$ (1) $56-44.0609+105.8483-5-120$ $36-44.8419-105.8505-2-15-$ $36-44.8491-10=0105-2-96-$ $56-44 \cdot 1072-105.3636-2-15-$ $06-44 \cdot 1594-105.5077-2-14-$

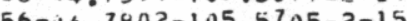

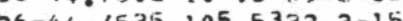

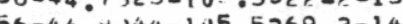
50 $44.02715-105.5050-2-15-$ $36-44.6715-105.5652-2-15-$ $56-44.06910-105.5123-2-15$ 56-44 00ub-105.308n-2-14 56-44.000 105.808 - $2-14$ $30-44.0669-105.665 n-2-14=$ $56-44.6521-105.6703-?-19-$ 56-44.0347 $105.6333-2-14-$ $56-44.8325-105.6494-2-15=$ $56-44.5183-105.6041-2-14=$ $56-44,8413-105.5216-2-15-$ $36-44$, o $15-105.5416-2-15$ (56-44. $56-44$. $y 1811-105 \cdot 5116-2-15$ $36-44$. $56-44,9363-105.617>->-15$ $36-44.9466-105.5916-2-15$ $56-44.8952-105 \cdot 5255-?-15$ $36-44,9569-105.4883-2-15$ $56-44 . y 6 d n-10^{5} .5277-2-15$ $36=44, y 883-105,4847-2-15$ $56-44$.5? 2 ? $-105.6786->-97$ $56-44.5427-105.6713-2-14$ $56-44,5372-105,72,8-2-14$ $56-44.5163-105.7$ J $363-2-15$ $56-44.558)-105.6436->-14$ $36-44,3644-105.7231-2-13-$ $56-44,2872-105.7883-?-13-$ $36-44.2983-105.837 ?-2-96-$
$56-44.2827-105.835 ?-2-13-$ $56-44.2827-105.835 ?-2-13-$ $56-44,1327-105.6505-2-14-$ $56-44,190 ?-105,72 ? 7-2-14=$ $56-44.2055-105.6236-7-14-$ $56-44 \cdot 2 ? 2 ?-105.7369-?-14-$ $5 b-44,+472-105,4166-2-15-$ $56-44,7669-105.4069-2-97$ ग6 $-44,9861-105 \cdot 393 r i-?-97$ $36-44,9833-10=3444-?-96-$ $56-44,+344-10=3166-7-15-$

$-200141-04 / i 1 / 7 h-10-15-13 . n-$ - G.h- 260- $-200146-04 / 01 / 76-12-21-$ - - $11->00154-03 / 31 / 75-10=12$ i $-200213-04 / \pi 3 / 74-16-12=$ $0-200215-04 / 03 / 76-16-13-$ 1) $\rightarrow 00218-04 / 36 / 74-8=20-$

$(1-200>21)-04 / 06 / 74-8-14-$

$16-1-6-4-6-1-3-2-1-4-2-3-1--1--$ $11-? 00145-04 / 01174-12-15-$ $10-200149-04 / 01174-13-21-15011=-709-8000=$

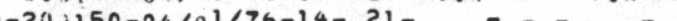
$400353-03 / 71 / 74-15-17-$

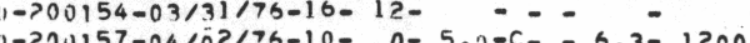
-

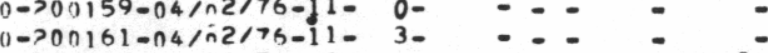
-200164-03/r2176-12- 4-5.10:- - 6.4- $3300-$ $n->00171-04 / 13 / 74-11-14-$ $=00172-94 /$ n $3 / 7 h-11-11=$

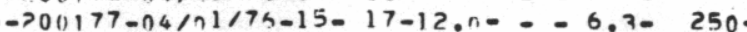
- $20 n 18 h-04 / 33 / 7 h-11-11-8 \cdot n=-6.5-20 n 0-$ -201188-04/n3/75-12-13-8.0- - - $6.5-6000$ $-200192-04 / 133 / 7 k-14-10-12 . n-2-6.1-490$ $\rightarrow 00193-04 / 03 / 76-14=15-15$

$-200200-04 / n 4 / 7 h-9-12-R_{0} n-=-6.8-1600-$ $-200203-04 / \cap 3 / 76-13-13-$ - $200204-04 / 73 / 75-13-11=$ $\rightarrow 00308-04 / 133 / 76-15-11=$ $->00310-04 \ln 3 / 74=15-14-$

$-207252-04 / 06 / 7 h-9-16-$

$->00224-04 / 106 / 74-9-10-$

$->0 n>25-n 4 / 14 / 74-10-20-$

$-200227-04 / n 4 / 7 h-11-16-10011-=-6.4-1500=$ $1-200228-04 / 14 / 7 h-11-18-13.01=-6.30170-$ $1->00>30-04 / 14 / 7 h-12-22-$

$-25.5-90=$ $\rightarrow 00335-04 / 14 / 7 h-13-25-17.18$

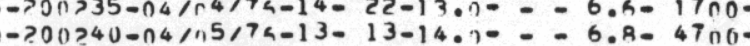
$->0 n \geqslant 4 ?-n 4 / 105 / 76-14-13-$ $12-1-6-4-6-=-2-4-3-4-1--1=-$
$6-1-6-4-6-1-2-4-2-4-1--1-$ $10-1-6-4-6-1-2-2-1-4-2-4-1--1=$
$10-1-6-4-6-1-2-2-3-4-2-2-2--1-$ $10-1-6-4-6-1-2-2-3-4-2-2-2--1=$
$12-1-6-4-6-1-2-4-3-4-3-2-2--1=$ $16-1-6-4-6----4-3-4-2--1--$ $9-1-7-4-7-\ldots--4-2-2-1--1-=$ $12-1-7-4-7----4-3-2-1=-1-$ $12-1-6-4-6-1-2-3--4-2-2-3--1=$ $9-1-6-4-6----4-3-2-3--10$ 2-1-6-4-6- - - - -4-2-2-3- -1$9-1-6-4-6-1-2-1--4-2-2-3-2-1=$ $12-1-7-4-6-1=-4-3-2-1=-9-1=$

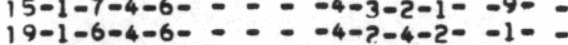
$12-1-6-4-6-1-2-3--4-3-2-2--1-$ $6-1-7-4-7-1-2-2--4-3-2-1--1=$ $80-1-6-4-6-1-3-2--4-2-2-1--1=$ $6-1-6-4-6=1-2-2-3-4-2-3-1--1=$ $6-1-6-4-6-1-2-1=-4-3-2-1=-1-$
$17-1-7-4-7-1-2-1=-4-3-2-1=-3-$ $10-1-7-4-6-----4-2-3-1--9-$ $10-1-7-4-6-2--4-2-2-1=-1-$

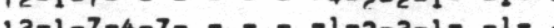
$12-1-7-5-6-\cdots--4-2-2-1--1-1$ $12-1-7-5-6-1--4->-2-1=-1-$ 15-1-7-6-7- - - - -4-2-2-1- -1-

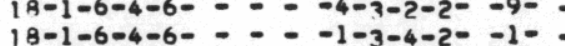

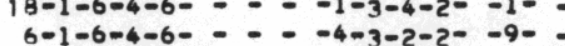
$6-1-6-4-6-\div-1-4-3-2-2--9-$
$9-1-6-4-6-\div-0-3-2-2--10$ $9-1-6-4-6-1-1-4-3-2-2--1-$
$12-1-7-4-7--4-3-2-1--1-$ $16-1-6-4-6-1-2-1--4-3-2-1--1=-$
$16-1-6-4-6-1-3-3--4-3-2-1--1=$ $16-1-6-4-6-1-3-3--4-3-2-1--1-$ $16-1-6-4-6--1--3-2-3-1--1=-$
$9-1-6-4-6-1-2-1--3-2-2-1--1=$ $9-1-6-4-6-3-3-1--4-3-2-1--1$ $10=1-t-4-6-1-2-1--4-3-2-1--1-=$ $18-1-7-4-7-1--4-3-2-1=-1-:$
$8-1-6-4-6-1-2-1=-4-3-2-1-1-1-$ $8-1-6-4-6-1-2-1=-4-3-2-1=-1-$
$6-1-6-4-6-1-2-1--4-2-2-1=-1-$ $12-1-6-4-6-1-2-1--4-2-3-1--1-=$ $12-1-6-4-6-1-2-1--4-3-3-1--1-=$ $16-1-6-4-6-1-2-1--4-3-4-1--1=-$ $13-1-6-4-6----4-3-2-2--1--$ $9-1-6-4-6-1-2-1--4-3-2-2--1=$
$9-1-6-4-6--1-$ $12-1-6-4-6----4-7-2-2--1-=$ $9-1-7-4-7-\div--4-3-3-3--1-=$
$9-1-7-4-7-\div-4-3-2-3--1=$

3.30

4.10

3.70

5.10

4.00

4.00
2.50

2.50
2.80

2. 80

3.00
4.50

4.50

3.10

3.10

3.40
3.40

3.90

4.00

3.60

3.80

3.10

3.80

2.90

3.80

3.70

3.10

3.40

3.80

4.30

4.10

3.00

2.80

3.70

3.40

3.40

3.90

3.30

3.10
3.60

3.30

3.00

3.00

3.70

3.30

6.00

5.00

2.80
3.50

2.90

3.00

-201)797-n4/n6/7a-11-22- … -

$1=200305-04 / n 6 / 75-14-19-$

$\div-$

3.30 
LASL Uranium Hydrogeochemical and Stream Sediment Reconnaissance Data Listing

\begin{tabular}{|c|c|c|c|c|c|c|c|c|c|c|c|c|c|c|c|c|c|c|c|c|c|c|c|c|c|c|}
\hline \multirow[b]{3}{*}{$\frac{a}{5}$} & \multirow{2}{*}{\multicolumn{5}{|c|}{ ERDA SAMPLE NUMBER }} & \multirow{2}{*}{\multicolumn{3}{|c|}{ TIME SAMPLED }} & \multirow{3}{*}{ 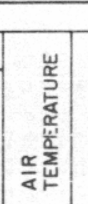 } & \multirow{3}{*}{ 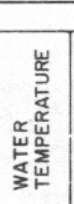 } & \multirow{3}{*}{ 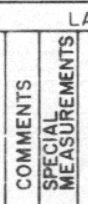 } & \multirow[b]{3}{*}{ I } & \multirow[b]{3}{*}{ 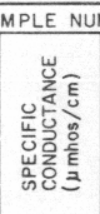 } & \multirow{3}{*}{ 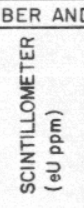 } & \multirow{3}{*}{ 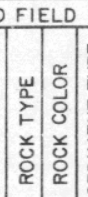 } & \multirow{3}{*}{ 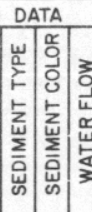 } & & \multirow{3}{*}{\multicolumn{2}{|c|}{ 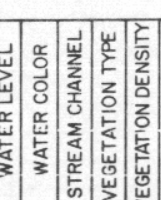 }} & \multirow{3}{*}{\multicolumn{2}{|c|}{ 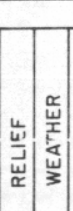 }} & \multirow[b]{3}{*}{ 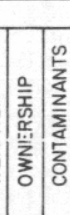 } & \multirow{3}{*}{ 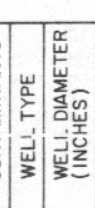 } & & & J \\
\hline & & & & & & & & & & & & & & & & & \multirow{2}{*}{ 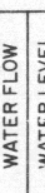 } & & & & & & & \multirow{2}{*}{ 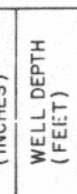 } & \multirow{2}{*}{ 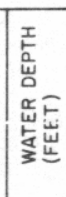 } & \multirow{2}{*}{$\begin{array}{c}\text { SEDIMENT } \\
\text { SAMPLES } \\
\text { UNITS } \\
\text { IN } \\
\text { Ppm }\end{array}$} \\
\hline & 㞻 & 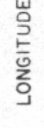 & 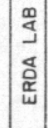 & 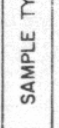 & $\begin{array}{l}\frac{\omega}{4} \\
\frac{d}{a} \\
\frac{a}{\alpha} \\
\alpha\end{array}$ & 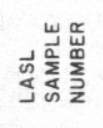 & 占 & 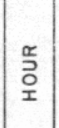 & & & & & & & & & & & & & & & & & & \\
\hline
\end{tabular}

$56-44,7530-105 \cdot 395>-7-15-$ $36-44.1890=105.8233-2-12$ $56-44.0111-105.9469-2-1 b$ $56-44.7702-105.8147-2-14$ $36=44.0711-105.8305-?-13-$ $56-4439050105.435 \%-2-470$ $56-44$. $9205-105.4352-2-97-$ $56=44.0803-105.6091-2-15$ $76-44.7680-105.6777-2-15$

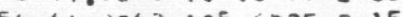
$56-44.7263-10=.6325-?-15$ $56-4.3105=10504438-2-15=$ $56-44.3165=105.4438-20-15=$ $36-44.3135-109.6669-2-14$ $56-44.3244-10=9063-2-14$ $36-44.3194-10=0016-2-14$ $36-44,3327-105 \cdot 7008-?-14-$ $56-44,3469-10=.7294-2-11$ $36-44,290>-105.6338-2-14=$ $56-44$. $2072-104.6316-2-12$ $56-44$. $1941-104.6311-?-12$ $56-44.2769-106.5647-2-14$ $56-44.0083-102.7266-2-14$ $36=44.0663-105.7115-2-14$ $56-44.0761=105.5416-2-15$ $56-44.0877-105.5$ y $25-2-12=$ $56-44.1633-10 \div .5394-2-96$ $56-44.7655-106 \cdot 2677-2-11-$ $56-44$. $9916-104.3116-2-15$ $36=44$. $97011-106 \cdot 2925-2-14$ $56-44,9594=104.2866->-15=$ $26-44,7591-106.2841-2-15$ $56-44.9638-10 \% \cdot 3244-2-15$ $56-44$. $\rightarrow 866-106.477 ?-?-15$ $56-44.4872-105.6033-?-9 i$ $56-44.375 d-10=0.4533-2-11$ $56-44.4019-105 \cdot 3813-2-11-$ $36-44,443:-105 \cdot 3847-2-11$ $56-44,4555-105 \cdot 4100-7-11$ $56=44 \cdot 4738-105 \cdot 3463-2-15$ $56-44 \cdot 4156-105 \cdot 3616-2-15$ $56-44 \cdot 4(194-10 \%=3236-2-15$ $56-44.3938-105 \cdot 3030-2-1$ b-
$36-44.3813-105: 3016-2-15-$ $36-44.3813-10 \div .3016-2-15-$ $26-44.648 r_{1}=10 \% .1355-2-15-$ $56-44.6311-106.1502-2-97-$ $56-44.7197-106.2533-2-15-$ $36-44.7047-106.2913-2-11$ $56-44.6944-100.3205-2-15=$ $36-44.6838-10+.3427-2-15=$ $56-44.0513-106.3425-2-15=$
$\Rightarrow 00310-04 \sin 6 / 76-16-14-$ -9.i1)=-6.3->3100=

$\rightarrow 00315-04 /$ is/7n- $9-14-10.11--5.7-310-$ $-200327-04 / 76 / 75-9-18-17.0-=-6.3-350-$ $-200347-04 / 10 / 74-120$

$-\mathrm{C}-$ $->0$ ) $752-04 / n 6 / 75-16-14-$ $-200355-04 / 17 / 7 h-9-12-$ (150 $\rightarrow 00361-04 / i 7 / 7 h-10-12-$ $\rightarrow 00362-04 / n 7 / 76-11-16-$ $\rightarrow 00371=04,0,6 / 75-12$

$-c^{-1}-$

$-c-$

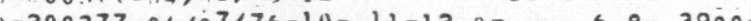
$-200378-04 / 77 / 76-10-11-12.0-=-5.50230-$ $->00379-04 / 47 / 75-11-13-11.0-=-5.9-3200-$ $-20 n 38 n-n 4 / i 7 / 7 h-12-12-12, n=-5.9-470-$ - $200385-n 4 / n T / 7 h=14-14-13 . n=-0.4-4700-$ (-20038h-04/n7/7h-14- $14-12.0=-0.1-7 n 0=$ $->00794-04 / 30 / 7 h-13-10-15.11--6.9-3950-$ $\rightarrow 200400-05 / h 1 / 7 h-11-18-16 . n--6_{0} \cdot k-2800-$ $->00403-04 / n 6 / 7 h-10$
$-200409-04 / n 6 / 76-11-13-10.5-0-6.4-390-$ (1) $-200432-04 / 20174-15$

$0 \rightarrow 200430-04 / 16 / 7 a-12$ -

(18-18010$0-200441-04 / 20 / 74-11-14-1>0+-0 . n-2500$ 1) $)>0(144>-04 / 70017 h-11=15-1200=-6.0=10000$ $n \rightarrow>00445-04 / 70 / 7 n-12-19-$ $n \rightarrow>00446-n 4 / 20 / 7 h-12-19-$
$n \rightarrow 200448-04 / 20 / 7 k-13-17-$ $n \rightarrow 200448-04 / 20 / 74-13-17-$
$(1)>017449-04 / 70 / 76-13-16-$ $-200454-04 / 12 / 75-17=19=$

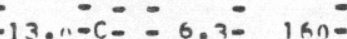
(1) $-200457-04 / 13 / 76-9-19-14.0--0.5-6000$ ) $11-200459-04 / 13 / 75-9-20-12 . n--0.1-175$ $11 \rightarrow 004662-n 4 / 13 / 74-10-20-$ $11 \rightarrow>00463-04 / 13 / 74-10-20-$ $\rightarrow 0(i 465-04 / 13 / 7 h-12-20-$ $n-200467-n 4 / 13 / 74-13-19-$
$1=-200468-n 4 / 13 / 74-13-19-$ i) $\rightarrow 000471=04 / 14 / 76-9-11-$ $\rightarrow 000472-04 / 14 / 76=9-13-$ $n=200474-04 / 14 / 7 h-10-13-$ $0-200476-04 / 14 / 74-11-15$ $0=200478-04 / 14 / 76-11-16-$ $1)=200479-n 4 / 14 / 7 h-11=15-$
$?=200481-04 / 14 / 74-12-15-$
$10-1-7-4-7---1-4-3-2-3--1=$
$9-1-6-4-6-2-3-1--4-3-3-1--1=$ $9-1-6-4-6---1-4-3-3-1--1-=$ $9-1-6-4-6-4-6-1-2-2--4-3-2-1--3-\div=$ $16-1-4-4-6-1-2-3--4-2-3-3--1-=$ $0-1-7-4-7=-10-0$ $9-1-7-4-7-2=-4-2-2-3-1-2$ $15=1-7-4-6=-0-4-2-2-3-1=$ 15-1-7-5-6- - - - 3-2-3-2- -1- 13-1-7-4-6- - - - 1-3-3-2- -1- $18-1-7-4-6-1--4-2-2-1--1-$ - - . - . - . - - $6-1-6-4-6-1-2-1--4-3-2-2--3-$ $3-1-6-4-6-1-2-1--4-3-2-2--1-$ $6-1-6-4-6-1-2-1--3-3-3-2--3-$ $10-1-6-4-6-1-2-1--4-3-2-2--3-$
$3-1-6-4-6-1-2-1--4-3-2-2--1-$ $13-1-6-4-6-1-2-1--4-3-2-2--3-$ $9-1-7-5-7-2-2-1-2-4-3-2-1=-1=$
$12-1-7-5-7-2-2-1-2-4-3-2-1=-1=$ $12-1-7-5-7-2-0-4-7-2-1--1=$ $18-1-6-4-6-1-2-1=-4-2-2-3--1-$
$12-1-7-5-7-1$ $9-1-6-4-6-1-2-2--4-2-3-3--1=$

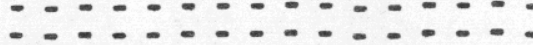
- - - - . - . - . - $12-1-7-5-7-2-2-1--4-2-3-1--1-=$ $9-1-7-4-7-2-4-2-3-1=-1=$ $13-1-7-4-7-2-0-4-7-2-1--1=$ $12-1-7-4-7-\cdots-4-2-2-1--1-=$ $12-1-7-4-7-=--4-2-3-1--1=-$ $10-1-7-4-7--0-4-2-1-1--1=-$ $12-1-6-4-6-2-3-1=-4-3-3-2--1-=$ $6-1-6-4-8-2-2-1=-4-3-3-1-1-1=$ $12-1-6-4-6-2-2-1-=4-3-3-1--1=$
$9-1-7-4-7-7-=-4-3-2-2--1=$ $9-1-7-4-7-\ldots--1-3-3-2--1-$ $9-1-7-4-7-0-0-4-3-3-2--1=$ $16-1-7-4-7=:=-4-3-2-2--1=$
$15-1-7-4-7=-$ $9-1-7-5-7-\ldots-4-7-3-3--1-$ $12-1-7-4-6=-4-2-2-3--1=$ $17-1-7-5-7-=-2=-4-2-2-3-1=$

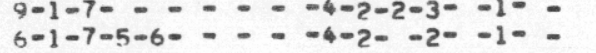
$7-1-7-4-6-1-4-2-2-2--1=$
$9-1-7-4-7-7-0$ 3.80
3.50 3.40 3.80 5.50

5.00

3.30

3.50
3.20
3.80

3.80

3.30
3.50

0.50

4.10

3.10

3.40

3.60

3.80

3.60

3.90

4.30

3.50
3.60

3.60

3.10

3.50

3.30
3.80

3.80

3.40

3.20
3.50

3.60

5.00

2.90

2.90

3.90

2.70

3.00

3.30

3.00

2.90

4.50

3.90

4.30

3.10

2.60

3.20
3.30 
LASL Uranium Hydrogeochemical and Stream Sediment Reconnaissance Data Listing

\begin{tabular}{|c|c|c|c|c|c|c|c|c|c|c|c|c|c|c|c|c|c|c|c|c|c|c|c|c|c|c|c|c|c|c|c|c|}
\hline \multicolumn{6}{|c|}{ ERDA SAMPLE NUMBER } & \multicolumn{26}{|c|}{ LASL SAMPLE NUMBER AND FIELD DATA } & U CONCENTRATION \\
\hline \multirow[b]{2}{*}{$\mid \begin{array}{l}\mid \frac{\omega}{5} \\
5\end{array}$} & \multirow[b]{2}{*}{$\frac{\mathrm{a}}{\mathrm{E}}$} & \multirow[b]{2}{*}{ 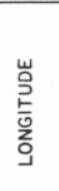 } & \multirow[b]{2}{*}{ 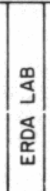 } & \multirow[b]{2}{*}{ 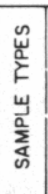 } & \multirow[b]{2}{*}{$\begin{array}{l}\frac{w}{b} \\
\frac{w}{d} \\
a \\
w \\
\alpha\end{array}$} & \multirow[b]{2}{*}{ 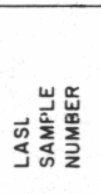 } & \multicolumn{2}{|c|}{ TIME SAMPLED } & \multirow[b]{2}{*}{ 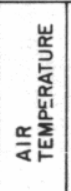 } & \multirow[b]{2}{*}{ 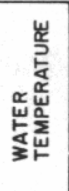 } & \multirow{2}{*}{ 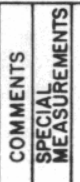 } & \multirow[b]{2}{*}{ I } & \multirow[b]{2}{*}{ 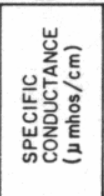 } & \multirow{2}{*}{ 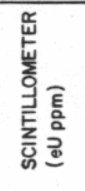 } & \multirow{2}{*}{\multicolumn{2}{|c|}{ 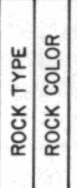 }} & \multirow{2}{*}{\multicolumn{2}{|c|}{ 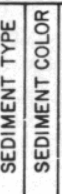 }} & \multirow{2}{*}{\multicolumn{2}{|c|}{ 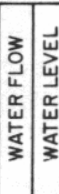 }} & \multirow{2}{*}{\multicolumn{2}{|c|}{ 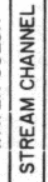 }} & \multirow{2}{*}{ 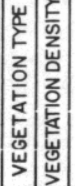 } & \multirow{2}{*}{\multicolumn{2}{|c|}{ 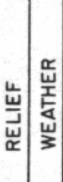 }} & \multirow{2}{*}{\multicolumn{2}{|c|}{ 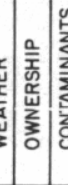 }} & \multirow[b]{2}{*}{ 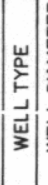 } & \multirow{2}{*}{ 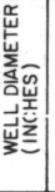 } & \multirow[b]{2}{*}{ 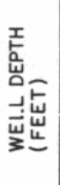 } & \multirow[b]{2}{*}{ 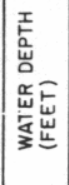 } & \multirow[b]{2}{*}{$\begin{array}{c}\text { SEDIMENT } \\
\text { SAMPLES } \\
\text { UNITS } \\
\text { IN } \\
\text { Ppm }\end{array}$} \\
\hline & & & & & & & 点 & $\begin{array}{l}0 \\
\text { o } \\
\text { a }\end{array}$ & & & & & & & & & & & & & 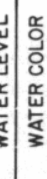 & & & & & & & & & & & \\
\hline
\end{tabular}

$56-44.0425-106.3166-2-97-$ $26-44.6405-104.3180-2-15-$ $56-44.0566-106.2936-?-14-$ ग6-44. $56-44.7327-106.3594-2-13-$ $56-44.1294-106.2919-2-15-$ $36-44$. $1975-10<.2850-2-15-$ $56-44.8283-106.3436-7-14-$ $36-44.8147-106.267 ?-2-14-$ $56-44.8338-106.2641-?-15-$ $36-44.0641-104.2294-2-15-$ $56-44.0661-100.2273-2-15-$ $56-44.7333-105.773 n-2-15-$ $56-44,7269-105.7766-7-15-$ $56-44$. $736-105.8194-2-15-$ $36-44.9883-105.8386-2-15-$ $56-44.7535-105.8080-2-15-$ $30-44.9347-10=.01017-i-15-$
$36-44.9755-105.9091-2-15-$ $26-44.9755-105.9091-2-15-$
$56-44.9419-105.9119-2-15-$ $56-44.9419-105.9119-2-15-$
$56-44.9266-105.9138-2-15-$ $56-44.7566-105.9138-2-15-$
$56-44.3888-105.903 n-7-15-$ $36-44.9661-105.9541-2-15-$ $36-44.7588-105.875 R-2-15-$
$56-44.7621-105.9172-2-15-$ $56-44.762 /-105.9172-2-15-$
$56-44.7919-105.9450-2-15-$ $56-44.1919-105.91450-2-15-$
$56-44.0160-105.9866-2-15-$ $56-44.8160-105.9866-2-15-$
$36-44.7769-109.870 ?-?-13-$ $56-44.0327-10^{5} .8741-2-15-$ $56-44$. $070 n-10=.9263-2-1$ 'j$36-44.786 y-106.1030-2-15=$ $56-44.1516-10^{4} .0733-2-15-$ $36-44.7630-10 t .0816-2-15-$ 56-44.0686-106.0513-2-15$56-44.033$ i) $-106.0519-2-15-$ $56-44.8141-106.0594-2-15-$ $56-44.8291-10 \% .0338-2-15-$ $56-44.7161-104.2347-2-15-$ $56-44.0650-104.1697-2-15-$ $26=44.6761-106.1913-?-15-$ $36-44.0988-106.2027-?-14-$ $56-44.4336-105.5741-2-15-$ $36-44.406 ?-105.5175-2-11-$ $56-44.393 n-105.6461-2-15-$ $56-44.3913-105.6467-?-15-$ $56=44.448 n-105.458 n-?-15-$ $56-44.4625-105.4294-2-11-$ $56-44.5183-105.4361-2-11-$ $36-44.4927-105.41,55-2-99-$ $56-44.4819-105.400 n-7-11-$
$56-44,5580-105.4705-2-49-$ $n-200482-04 / 14 / 76-12-18-\quad-C--$
$n-200483-04 / 14 / 74-12-18-\quad--$ $n=200484-04 / 14 / 7 h-12-16-15.0=-E-8=3900-$ $1 \rightarrow 00 \Omega 485-04 / 14 / 76-12-17-1500=-6.8=>900=$ $1) \rightarrow 00487-04 / 14 / 74-12-17-15.0=-06.9-450-$ $1=? 00488-04 / 14 / 74-13-17=$ $\Rightarrow 00492-04 / 14 / 74-15-17-\quad-$ - $_{-}-$ $-200493-04 / 14 / 74-15-16-15 \cdot n-=-6.4-2200-$
$-200497-04 / 14 / 74-16-17-15.0-=-6.3-2950-$ $n=200498-n 4 / 14 / 7 n-17-14-$ $11) \rightarrow 000499-04 / 14 / 76-17-15-$
$1 i)$
$-000500-04 / 14 / 76-17-14-$ $\rightarrow=00505-04 / 37 / 75-11-16-$ $1=>00503-04 \operatorname{157} 75-11=15-$ -? -

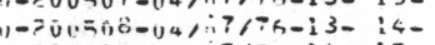
$n->00510-04 / 17 / 7 n-14-15-$ $n-200513-04 / n 7 / 7 n-15-15-$ 1) $-200514-04 / i 7 / 74-15-1$ -200518-nu/nt/7h-16-15(1) $-200521-04 / 08 / 75-14-14$ $1,-200525-04 / 68 / 74-15-11=$ $0=200524-04 / 78 / 74-14-14-$

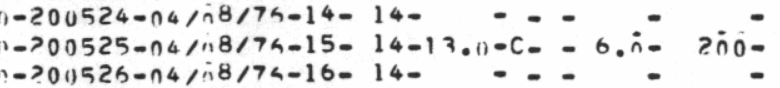
$\Rightarrow>0 n 527-n 4 / 78 / 74-16-18=$ -?00528-n4/n8/7h-17- 18 $n=200530-04 / 78 / 75-17-16-$ $n-200531-04 / 18 / 7 h-17=15-$ $n-20) 537-n 4 / n 9 / 7 k-11-14-$ n-? $000539-n 4 / 09 / 74-11=16-$ $n->00540=04 / i 9 / 74-12-22$ $n \rightarrow 00544-n 4 / i 9 / 76-13-25-$ $n=>00545-14 / n 9 / 76-14-20-$ $1-200549-14 / 29 / 76-15-18-$ $1=201,551-n 4 / n 9 / 74-15-16-14 . n--6.3-15 n 0-$ $-200556-04 / 10 / 7 n-10-8-$
$-200560-04 / 10 / 7 n-13-1 n-11, n=-E$ $\rightarrow 00568-04 / 10 / 76-16-1 n-$ $\rightarrow>07569-04 / 10176-16-9-$ $\Rightarrow 001575-04 / 12 / 7 h-8-12-$
$\Rightarrow 0010576-04 / 12 / 74-8-11-10 . n-C=-6 . n-8$ $1)>00580-04 / 12175-9-14-12.0-=-6.6-2000-$ $1-200583-04 / 12 / 75-10-14-\quad-C=-6=70-$
$n-200584-04 / 12 / 75-10-17-11,0=-0.3-700-$ ; $-200585-04 / 12175-10-19-\quad-C_{-}-$- $10-1-7-5-7-\cdots-4-2-2-3--1--$ $7-1-7-4-7-\ldots--4-2-2-2--1--$ $9-1-7-5-7-1-2-2--4-2--2--1--$ $9-1-7-4-7--1-2-4-2-2-2--1=$ $10-1-7-5-6-1-2-2--4-2-2-3--1-=$

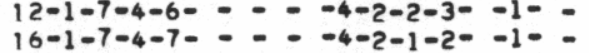
$9-1-7-4-7-1-7-4-7-1-2-2-4-4-2-1-2-4-10-$
$9-1-2-2-1-$ $9-1-7-4-7-1-2-2--4-2-2-2--1-$
$9-1-7-7-2-3-2--4-2-1-2--1-$ $9-1-7-7--2-3-2--4-2-1-2--1-=$
$9-1-7-5-7--0-04-2-2-2--1-=$ $9-1-7-4-7=--4-2-2-2--1=$

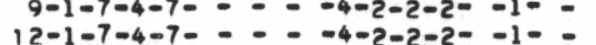
$13-1-7-4-6-\cdots-4-2-2-1--1-=$ 15-1-7-4-6- :- - -4-2-3-1- -1- $15-1-7-4-6-=-2-4-2-3-1=-1-=$ $10-1-7-4-6-2--4-2-2-1-10$

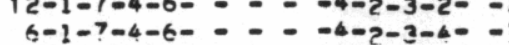
$6-1-7-4-6-:=-4-2-3-4--1$
$13-1-7-4-6-10$ $10-1-7-4-6-\ldots-4-2-2-3--1$ $12-1-7-4-6-\div=-4-2-2-3--1$
$13-1-7-4-6-1-$ $13-1-7-4-6-1-1-4-2-3-2--1$
$12-1-7-4-6-1$ 15-1-7-4-7- - - -4-3-3-2- -1

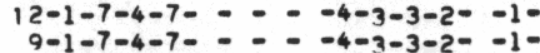
$9-1-7-4-7-=-2-4-3-3-2--1-$
$17-1-6-4-6-=-8-3-3-2--1-$ $12-1-6-4-6-1-2---4-3-2-2--1-$ $9-1-6-4-6---4-3-3-1--1-$ $9-1-6-4-6-1-4-4-3-2-1--1-$
$9-1-7-4-6-2-4-2-4-1--1-$ $12-1-7-4-6-0-4-7-2-1--1=$ $13-1-7-4-6----4-7-2-1--1-$

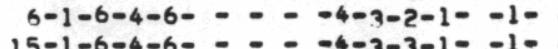
$15-1-6-4-6-2--4-3-3-1--1=$ $9-1-6-4-6-:=-2-4-3-2-1=-1-$
$9-1-6-4-6-=-2-3-4-1=-3-$ $9-1-6-4-6-=-2-4-3-3-2--1-$
$5-1-6-4-6-$ $15-1-6-4-6-2=-4-3-4-3--1-$ $13-1-6-4-6-----4-3-2-4--1-$
$9-1-6-4-6-1-2-4--4-3-3-4--1-$ $15-1-7-5-7-\cdots--4-2-2-3--1-=$ $6-1-7-5-8-2-3-1--4-2-2-3--1-$
$9-1-7-4-7--0$ $9-1-7-4-7-\div-=-4-7-2-3--9-$ $9-1-7-4-7---4-2-2-3--9-$ $15-1-7-4-7----4-2-2-3--1-$ $12-1-7-4-6-3-3-1=-4-2-2-3--1--$ $12-1-7---2-3-1--4-2-3-2--1--$ 9-1-7-4-6- $15-1-7-1-2-3-2--1-$ $15-1-7-4-6-2-2-1--1-2-3-3--1-=$
$9-1-7-4-7--0-4-2-2-2--1-=$
4.60

3.40 7.70 4.80 .90 4.50 3.70
4.20 4.20 4.4 2.90 48 6.70 5.20 40 2.80 3.30 3.00 (50 2.90 4.50 5.30 80 3.20 4.90 
LASL Uranium Hydrogeochemical and Stream Sediment Reconnaissance Data Listing

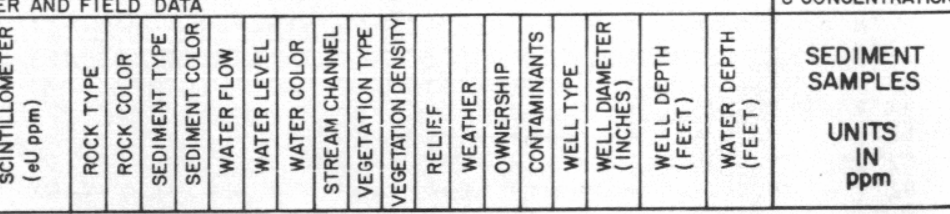

$56-44,5+10-105.405 n-2-15=$ $56=44.5086-105.4175-2-99-$ $56-44 .>741-105.4197-2-99-$ $36-44.6105-105.3997-5-11-$ $56-44.018)^{2}-105.4297-2-99-$ $36-44.0319-102.3005-2-15-$ $56=44.5455-105 \cdot 31>5-2-14-$ $36=44.525 ?-10=.2516-5-15-$ $36=44.6183-105.3022-2-15-$ $36-44.6194-105.9033-2-15-$ $36-44.6127-105.930 n-2-15$ $56-44.6327-105.9858-2-15-$ $56-44.6788-105.9761-2-15=$ $36-44.6797-105.9752->-15-$ $56-44.1383-105.9444-2-15-$ $56-44.6891-10 \% .0086-2-15-$ $56=44$. OHO3-10h. 0086-2-1 $56=44.0812-10 \% .0083-2-15$ $56=44.6683-10 \% .0783-2-12-$ $26-44.6460-10 k \cdot 0477-2-12=$ $56-44.0087-106.0094-2-15$ $36-44.3177-105.9344-2-15-$ $56-44.5361-1 \cup 6.1141-2-15-$ $56-44$. 5.J5?-1 UK. $1175->-15-$ $56-44$, ग82?-104. $1425-7-15-$ $36-4$. bius-1 ut. $173 h-2-97$ ग6-44. गत63-1 Uh. $1794-2-15-$ $56-44.590$ b- $106.1911->-15$ $36-44,5891-10 \%, 1822-2-15-$ $56-44$. ग4 $74-1$ Oस. $1736-?-13-$ $36-44$. ग177-10f. $1305-?-15$ $56-44.4716-10 \mathrm{H} \cdot 247 ?-?-15-$ $56-44.4944-104.2094-2-15$ $36-44.4902-10 f .3038-2-97-$ $56-44.4588-106.3116-2-97$ $36-44.4427-106.3244-2-11-$ $36=44.4177-1$ of. 3333-2-15. $56=44.4661=100.3416-2-13$ $56-44,4522-106.2855-2-15=$ $36=44.4961-106.2611-2-97-$ $56=44.4625-106.1075-2-97-$ $36=44.3544-10 * .1544-2-15$ $56-44 \cdot 3813-106,1805-2-15-$
$56=44.3408-10 \% .2133-2-15-$ $36-44,3333-104.2211-2-12$ $56-44.2788-10 \% .1$ ग0 n-2-15$56-44.253 n-10 \mathrm{f} .175 ?-2-15=$ 56-44.8300-10e. $2233-2-14$ $26=44.7594-106.22227-2-15-$ $56-44,1586-106.1772-2-15$

\section{$?-200586-04 / 12 / 74-10-19$} $-2019587-04 / 12 / 76-11-18$ (1) $=200588-04 / 12 / 76-11-21-$ - $0 . \bar{n}$ - 4ñjo - $200590472175-12-21$ $11-200593-04 / 12 / 7 n-13-28-14 \cdot 0=-0 . \bar{n}-100-$ $-200596-n 4 / 12 / 7 n-14-29$ $n \rightarrow 00500-04 / 12 / 7 a-16-26-$ $v=207403-04 / 37 / 74-12-18-$ ) $-700604-04 / 77 / 76-12-19-$ - $-200605-04 / n 7 / 74-12-16$ - $200506-04 / r 7 / 76-13-21$ $0 \rightarrow 2011607-04 / 37 / 7 a-13-21-$ 1-?00611-04/ $17 / 7 / 5-15-19-$ (1)>00616-04/119/76- 8- 15 (a) - $0517-04 / n y / 7 h-$ o- 15 150 $-203520-04 / 49 / 79-10-18-12.0=-6.4-70 n 0=$ $n=? 0) 624-04 / n 9 / 7 h-11-23-16 . n=-6 . k-42 n 0-$ $n=200631=04 / n 9 / 75-16-18-$ $n=200635-04 / 10 / 76-12-12$ 1) $=200538-04 / 10 / 74-13-12-$ $n=207539-04 / 10 / 76-13-12$ $\therefore=? 00644-04 / 12175-8-15$ $0-200545-04 / 12 / 74-9-15-$ $1=? 00646-04 / 12 / 76-9-17-$ 1) (1) $->00648-04 / 12174-9-15$

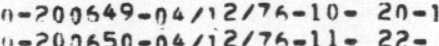
(1) $-200650-1) 4 / 12 / 76-11-22-$ $0=200653-n 4 / 12 / 76-15-25-$ - $200655-04112775-16-23-$ $n \rightarrow 00056-04 / 13 / 76-9-22-$
$n \rightarrow 00658-04 / 13 / 76-9-22$ $11=200661-04 / 13 / 75-12-22-9 . n-C-=6.5-4600=$ $11=>00663-04 / 13 / 79-13-22-$

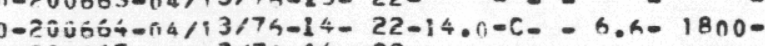
(1) $1=200666-144 / 13 / 76-14-22$ $0 \rightarrow>00670-n 4 / 13 / 76-14-18=$ $1-200472-04 / 14 / 76-10-11=$ $n=200674-04 / 14 / 76-9-16=$
$n \rightarrow 00675-04 / 14 / 76-10=16-$ 1->03576-04/94/7h-10-14-12.n- - 7.5- 9nno1) $-200583-04 / 14 / 7 h-12-16-$ $->00702-04 / 14 / 76-17-14-110-=-6.10400=$ $1=? 00705-04 / 15 / 76-8=10-\quad 0=-6010400$ $1=200706-04 / 15 / 76-8-10=$
15-1-7-4-7- - - -4-2-2-2- -1- 13-1-7-- - - - - -4-2-3-2- -1- 9-1-7-4-7- - - -1-2-3-2- -1- $3-1-7-4-7=-0=-1-2-3-20-1=$

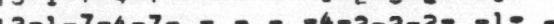
19-1 $9-1-7-5-7-1-2-2--4-2-2-1--1=$

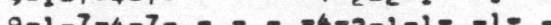
$9-1-7-4-7=-2=0$ $12-1-6-4-6-=0-4-2-2-2=-1=$ $9-1-4-4-6-=0=4-3-2-2--1=$ $19-1-6-4-6-=-0-4-3-2-2--10=$ $16-1-6-4-6-\ldots--4-3-2-2--10=$

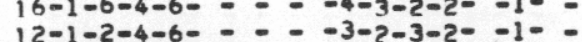
$12-1-6-4-60-0-4-3-2-1--1=$ $12-1-6-4-6-12-6-4-6-0-4-3-2-1--1=$ $13-1-6-4-6-1---3-3-2-1--i=$ $16-1-6-4-6-1-2-1-2-2-2-2-1--1-$ $12-1-6-4-6-2-2-1--2-2-2-1--3-=$ $12-1-7-4-7=---4-3-2-1--1-$ $19-1-6-4-6-=-2-2-4-2-1=$ $9-1-6-4-6-0=0$ $16-1-7-4-7-2-3-2-4-3--1=$ $901-704-7=0-0=0$ $16-1-6-4-6=-1-3-2-2-2--1=$ $16-1-6-4-6-\cdots--4-3-3-2--1=$ $16-1-6-4-6-1=-3-2-3-2--1=-$
$12-1-6-4-6-3-2-3-2--1=$ $12-1-6-4-6-=-1-3-2-3-2--1-$
$16-1-6-4-6-$ $16-1-6-4-6-2--3-2-3-2--1=$ $12-1-7-4-7-1-3-2--3-2-3-2--1=-$ $12-1-6-4-6----3-2-3-2--1-=$

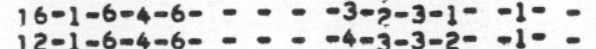
$12-1-6-4-6-1-0-4-3-3-2--10-$
$9-1-6-4-4-\div-3-2-3-3--10$ $9-1-6-4-4-1-0-3-2-3-3--1=-$
$12-1-6-4-6-0-3-3-3-2--1-=$ $12-1-6-4-6--2-1=-3-3-3-2--1-=$ $14-1-6-4-6-2-2-1--3-2-4-2--9-$
$16-1-6-4-6--$ $16-1-6-4-6--1-1-4-4-3-2--1=-$
$12-1-6-4-6-1-3-1=-3-4-3-2--1=-$
$16-1-6-3-6--6--3-4-3-2--1=$ $16-1-6-3-6--1--4-4-3-2--1=$
$16-1-6-4-6-2-3-1--4-3-3-2--1=$ $16-1-6-4-6-2-3-1--4-3-3-2--1=$
$16-1-6-4-6--0$ $16-1-6-4-6-1-0-3-2-3-2-1-$
$16-1-6-4-6-$

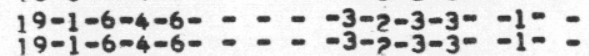
$9-1-6-4-6-1-2-2--3-2-3-2--1-$ $12-1-6-4-6--\cdots-2-2-2-2--1-=$ $19-1-1-4-1--0-3-\dot{2}-3-1--1--$ $12-1-7-5-7-1-2-2--4-2-2-2--1=$
$9-1-6-4-6--1-$ $12-1-6-4-6-\ldots-4-3-2-1--1--$ $\mathrm{ppm}$

2.20

3.00

3.10

4.10

?

7

3.40

3.4

4.90

4.80

.

3.9

3.10

3.70

4.2

4.6

4.90

4.8

3.6

.40

4.70

.20

4.10

4.00

3.70

3.60

3.70

3.60

4.30

3.30

3.70

4.50

.00

2.80

4.30

3.50

3.60 
LASL Uranium Hydrogeochemical and Stream Sediment Reconnaissance Data Listing

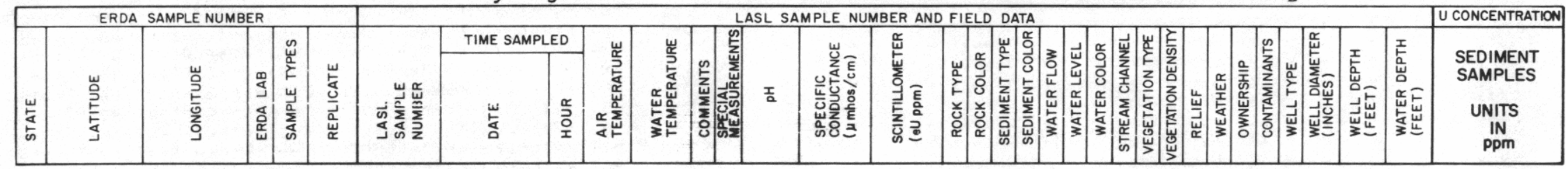

$56-44.1977-106.1580-2-15-$ $56-44.0 ?+1-106.1783-2-15-$ $30-44.7111-106.3486-?-15-$
$56-44.0965-100.3736-i-15-$ $36=44 .+361-104.3591-2-15$ $36-44,7393-104.3588-?-15-$

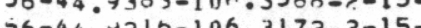
36-44. $36-44$. $7355-104.170 n-?-15-$ $36-44.9308-106.1833-2-15$ $56-44.9003-106.1408-2-15$ $56-44$. $7100-106,1386-2-15-$ $36-44.7916-1$ Uh. 3 अ22-2-97$56-44.1980-106.4205-?-14-$ $36-44.0138-106.3333-2-14-$ $56-44.0388-10 k .4327-2-97-$ $56-44.0522-106.4894-2-1 b-$

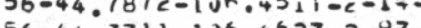
$56=44.7711-100.4527-2=97-$ $56-44.7794-102.4327-2-15-$ $36-44.7560-106.3527-7-15-$ $56-44,7727-10 \mathrm{~N} \cdot 3955-2-15-$ $56-44$. $1911-105.8383-?-15=$ $56-44.6583-104.3727-2-15$ $26-44.0741-100.4561-2-14=$ $56-44.1141-1 \cup 4.4336-?-15-$ $36-44.1116-10 \mathrm{OR} .4 \mathrm{H6} 1-2-15$ $56-44,1152-106.4261-2-14=$ $56-44.6852-10 \mathrm{R} .4119-2-14-$ $26-44.0438-104.4077-?-97-$ $56-44.0488-106.4638-2-14-$ $36-44.5988-106.4233-2-15-$ $36-44.5766-106 \cdot 4155-2-15$ $36-44.585 n-106.4058-2-15$. $36-44.5588-106.4325-2-15$ $56-44.5522-10^{h} .4552->-15$ $36-44,5208-106.4113-2-14-$ $56=44,5886-106.4583-2-14=$ $36=44.5355-10 f \cdot 535 c-2-15$ $56-44.5475-104.6194-7-15=$ $56-44.6761-106.5116-2-14-$ $36=44.6866-106.5397-7-14-$ $56-44.7100-10 \mathrm{~K} .5344-2-11$ $56-44,7111-106.5558-2-11-$ $36-44,1180-106.5638-?-11$ $50-44.7>16-106.575 n->-99-$ $56-44.6455-106.55(1) n-2-15-$ $56=44.0416-106.5283-2-11$ $56-44.04 y 1-104.5063-2-14-$ $56-44.6997-106.6127-2-15-$
$56-44.7177-104.6097-2-15-$
$0=700708-04 / 15 / 76-8-14-$ $-207709-04 / 15 / 76-9-15-$ $0.00711-04 / 15 / 7 h-11-17-$ 007100 - $200714-04 /$ istan-11- 18 - $00715-0475174-11-18-$ $00710-0415 / 76-12=10-$ $0-200719-04 \pi 15 / 76-12-20-$ $0-200720-144 / 15 / 74-12-22-$ $0-200722-04 / 15 / 75-13-21-$ (1)>07723-04/15/76-13-21!) $-200725-04 / 16 / 7 h-8-5-\quad-C_{-}-5_{-}^{-}-$

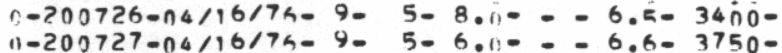
$11-200727-04 / 16 / 74-9-5-6.1:-0-6.6-3750-$
$0-200728-04 / 16 / 74-9-40-0-0$ a-200728-n4/16/74- 9-

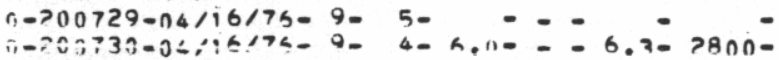
$0,>00731-04 / 16 / 75-9-5-$ $-200732-04 / 16 / 7 n-90$ - 6 $2=200733-n 4 / 16 / 74-10-$
$0=200735-04 / 16 / 74-10-$ $0-200735-04 / 16 / 76-10-$
$0 \rightarrow 00737-04 / 19 / 74-9-9$ $0 \rightarrow>00741-04 / 71 / 74-10-13$

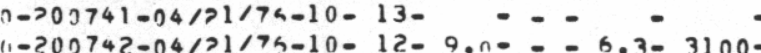
11-200742-04/>1/7h-10- 12

ij $\rightarrow 00744-04 / 21 / 74-10=10-0$

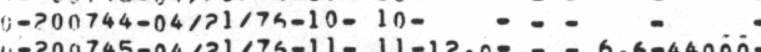
$11=200745-04 / 21 / 75-11-11-1>\cdot 0=-6.5=440000-$ 1-200746-04/21776-11-13-12.0- - - 6.9-16000$n \rightarrow 00750-04 / 21 / 7 n-12-16-15 . n=-0.9-290 n-$ (i) $\rightarrow 000751-04 / 71 / 76-13-14-$ $0=>00753-14 />1774-13-14=$ $0-200754-04 / 21 / 76-13-14-$ (i) $-200755-04 / 71 / 75-14-16-$ $n=200757-04 / 21 / 76-14-14-$

$n->00761-04 / 71 / 7 a-15-14-11, n-=-6.3-1700-$ 1) $-200763-04 / 21 / 74-15-15-12.00=-6.3010000-$ ? $->00765-04 />2 / 75-9-14$

$0->3 C 769-n 4 / 22 / 75-11-20-\quad-C-=-160-$ $-20 n 772-04 / 22 / 74-12-20-13 \cdot n-2-6.6-1600-$ $->00773-14 / 22 / 76-13-18-17 . n-0-6 . n-150-$ $\Leftrightarrow 00774-04 / 22 / 76-14-21-150 n--5.7=45=$ $1=20 n 775-04 / 22 / 76-14-21-17.0--6 . n-1150-$

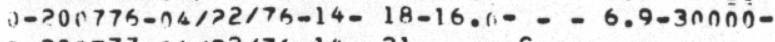
$0-300777-04 / 25 / 76-14-21-$

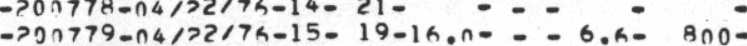

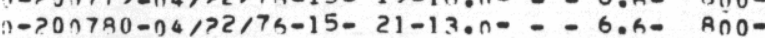

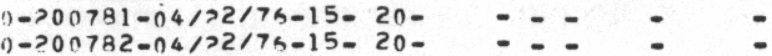

$12-1-6-\div-0-4-3-2-1--1-=$ $15-1-6-6-2=-4-3-2-1--1=$ $9-1-6-4-6=-=-4-3-2-1=-1=$ $9-1-6-4-60=--4-3-3-1=-1=$ $10=1-6-4-60=0-4-3-3-1=-1-=$ $10-1-6-4-6-2=-4-3-3-1--1=$ $3-1-6-4-6-0=-04-3-3-2=-1=$ $7-1-6-4-6-2=-0-4-3-3-2-2=-1=$ $10-1-604-60-0=-4-3-3-2=-1=$

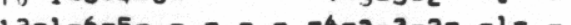
$12-1-6-5-2-1-4-3-3-2-1=$ $9-1-6-5-6=0=0-4-3-3-2=-1=0$ $9-1-7-5-7-\ldots=-4-2-3-3-1=$ $15-1-7-1-10$ 1-7-5-7-- - - -4-2-3-4- -1- $9-10-7-4-7=0=0-10=0$ $9-1-7-4-7-=-8-2-3-4=-1=$ $9-1-7-5-7=0-0$ $18-1-7-5-7-1-1-7-4-7-2-2-4--1=$ $13-1-7-4-7-1=-1-7-3-4--10=$ $10-1-7-5-7-\ldots-04-2-2-4--10=$

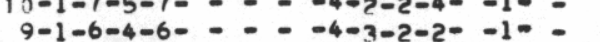
$12-1-6-4-6-\cdots-4-3-2-2--10-$ $18-1-6-4-6-1-3-1--4-3-2-2--1-=$

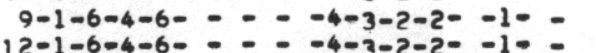
$12-1-6-4-6-1-3-1--4-3-2-2--1=-$
$9-1-6-4-6-1-3-1=-4-3-2-2-1-$ $6-1-6-4-6-1-3-1--4-3-2-2--1-=$ 15-1-6-4-6- - - - -4-3-3-2- -1- $6-1-6-4-6-1-2--4-3-2-2--1-$
$9-1-6-4-6-0-4-3-3-2-1-$ 9-1-6-4-6- - - -4-3-3-2- -1- $9-1-6-4-6-=-1-4-3-3-2-1=-$ $10-1-6-4-6-\ldots--4-3-2-2--1--$ $15-1-6-4-6----4-3-3-1--1=$ $6-1-6-4-6-1-3-1--4-3-3-1--1-$
$9-1-6-4-6-1-3-1--4-3-3-1--1-$ $9-1-6-4-6-1-3-1--4-3-3-1--1-=$
$9-1-7-4-6-1-7--4-2-2-2-1-=$

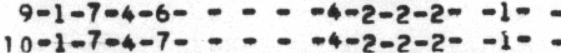
$9-1-7-5-7-3-3-1=-4-2-2-1--1-=$ $10-1-7-5-7-1-2-2--4-2-2-1<-1=-$
$9-1=7-5=7-2=2-1--4-2-3-2--10=$ $9-1=7-5=7-2=2-1-=4-2-3-2-1-1=$ $9-1-7-5-7-2-2-1--4-2-3-2--1=$
$10-1-7-5-7-2-2-1--4-2-3-2-1-$ $10-1-7-5-7-2-2-1--4-2-3-2--1-0$ $10-1-7-4-7----4-2-3-2--1-=$

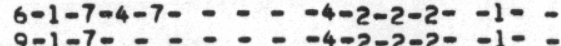
$3-1-7-5-7-2-2-2--4-2-2-2--1-$

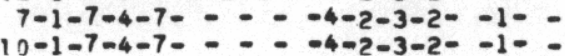

4.0

4.10

3.70

3.40

3.60

3.60

3.20

3.20

3.50

11.20

3.90

3.50

3.40

2.60

3.60

3.40

3.30

2.40

3.60

3.60

3.40

4.20

3.00

3.70

3.50

3.00

3.50

3.30

2.90

2.60

3.50

3.30

3.50

3.50

2.90

3.40

3.00

3.30

3.10

.60

3.00

2.90

2.90

3.00

2.50
2.80 
LASL Uranium Hydrogeochemical and Stream Sediment Reconnaissance Data Listing

$50-44.0911-106.5709-2-14-$ $\begin{array}{ll}0 & -14\end{array}$ $56-44.5577-106.5033-?-15=$ $36-44.1355-106.6341-2-11=$ $56-44,156)=106.6925-?-14=$ $56-44.7991-106.7441-2-140$ $36-44.7869-106.6827-2-14-$ $36-44.7802-106.6038-2-15$ $56-44.7647-106.6415-2-140$ $56-44.7516-106.6033-2-11$ $56-44.0077-10 \% .6802-2-15$. 6 $=44$ d0 d6-1 ve. $668 R-2-15$ $56-44.8077-10+.6702-2-15$ $36-44.7447-10 \% .6155-2-11$ $56-44.4283-10 t, 5936-2-15=$ $36-44=47-114,4078-2-12$ $56-44.4913-1$ Uf. $8033-2-12=$ $36-44.2788-106.7263-2-15$ $56-44.2711-106.7594-2-11$ $56-44.2597-104.8013-?-11$ $56-44.2605-106.8025-?-15$ $56=44.2766-106.7655-2-12$ $36-44.2469-10+.805 n-2-12$ $56-44.3083-106.8144-2-11$ $56-44$. 2स33-106.7861-?-11 $26-44$. $2758-106.8050-2-12$ $56-44$. $3288-106.6672=?-12$ $36-44.2636-106.6377-2-11-$ $36-44.2269-104.6209-2-11$ $36=44.2813-10 \% .6263-2-12=$ $36-44.3097-106.6325-2-15=$ $56-44.8402-10 f .8983-2-12-$ $56-44.8702-106.9683-2-15-$ $56-44.8472-106.8736-2-11=$
$56-44.8527-106.8800-?-15=$ $36=44.4425-106.9911-?-15$ $56-44.8472-106.9 \rightarrow 77-2-11$ $56-43.6005-106.5238-2-14-$ $36-43.5997-106.5216-2-97$ $56-43.6122-106.4994-?-97=$ $56-43.6447-106.467 ?-?-14=$ $36-43.6197-106.3488-?-15-$ $56-43.0288-106.4266-2-97-$ $36-43.6283-10 \mathrm{f} .4227-2-15$ $56-43.6361-106.3727-2-15-$ $36-43.6188-106.3594-2-15-$ $36=43.0188-106.3261-2-15$ $56-44.7930-106.6136-?-15-$ $56-44 \cdot 7611-10+.5336-2-11-$
$56-44.628 n-106.5652-2-15-$
$-200785-04 / 22 / 75-16-18-14 \cdot 0--6.8-1200-$

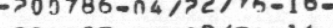
$1-200787-n 4 / 72 / 76-16-18-15 \cdot 0-=-6.3-1250=$
$n \rightarrow 200788-04 / 72 / 76-16-19-$

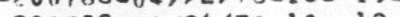

$0-200789-0472475-10-19-9.0=-6.5-1100-$

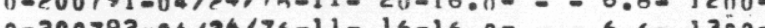
$0-200792-04124176-11=16-16.00=-6.4=1300=$

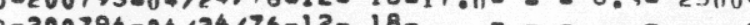

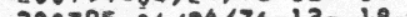

$0-200790-1404040-2150-$ $0-200796004 / 24175-12019-16.0=-6 . \mathrm{h}=13 \mathrm{nO}$ $0-200797-04 / 24 / 76-13-20-$

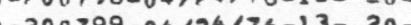

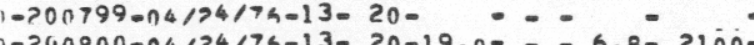
$->00804-04 / 22 / 76-13-21-$ - - $-2 n \cap A n 7-n 4 />2 / 74-16-17-14 . n=-0.8-125 n=$ $=>00808=n 4 / 22 / 74-17=16-12.5-\cdots-6.9=7 n 0-$ $\rightarrow>00 A 11-04 / 73 / 74-11-10-$

$1-200812-04 / 23 / 74-11-10-6.0-C_{-}-6.5-24000-$ $\rightarrow>0 n R 13-n 4 / 23 / 7 R-12-10=8.0=-6.3-1100-$ $\rightarrow-201.914-04 / 73 / 76-12-11-$

- $000215-04 / 73 / 74-15-12-10.00-0-6.4-2150=$ $\rightarrow$ - 0 ) $916-04 / 23 / 74-15-8-10.00-0.5-1350-$ $0-200917-n 4 / 33 / 75-16-8-100 n=-0.5=950-$ $11=200818-04 / 23 / 76-16-10-9.0-=6.4-18000-$ 1) $-2008919-04 / 23 / 7 h=17-12-9.00--6.3-1900-$

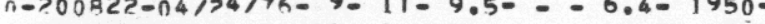
0-?00823-04/24/74-11-10-15.n- - 6.5- 750$-2019224-04 / 24 / 7 h-12-21-21.0--0.8-1000-$ - $00325-04 / 24 / 74-15-19-16.00-$ - 5.90 900$1=? 00256-04 / 24 / 74-16-11-$ -20กR31-04/26/7h-13-8-9.n- - - 6.4- $16000=$ $0-200332-04 / 26 / 75-15-9-950=-6.30950$ $-200833-04 / 76 / 76-13-16-8 . n=-5=6.3-950=$
$-20 ก 834-04 / 76 / 76-16-8-80$ $-200934-047>26776-16=$

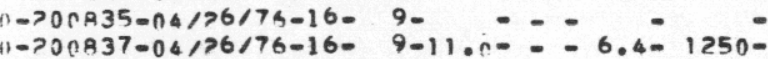
$1=200837-06 / 26 / 76-16-9-11 \cdot 0-=-6.4-1250-$

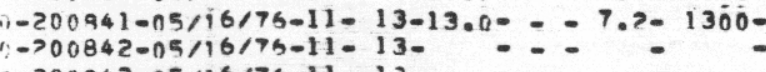

$-200943-05 / 16 / 76-11-13-$

(1-200844-05/16/76-12-16-16.0-C- - 7.8-11500(1-20ก945-na/16/75-12- $18-$ $n=20,146-n 5 / 16 / 76-13-16-$
$1=200847-n 5 / 16 / 76-13-16-$ i) $-200848-05 / 16 / 7$ h-13- 18 $0 \rightarrow>00349005 / 16 / 76-14-18-$ (i) $=200850-05 / 16 / 75-13-18$ $1->00351-n 4 / 24 / 76-13-20-$ $0=200852-04 / 24 / 76-14-20-11,0=-6.7=700=$

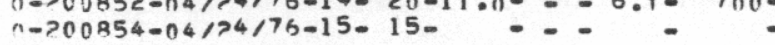

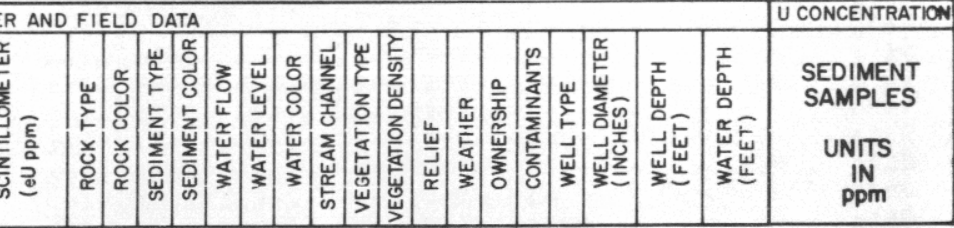

$7-1-7-5-7-3-3-2--4-2-2-2--1-\div=2.79$ $12-1-7-5-7-2-2-2--4-2-2-2--1-=$ $4-1-7-4-7-1-2-3--4-2-1-2--1--$ $9-1-6-4-6-2-2-1=-4-3-3-1=-1-=$ $12-1-6-4-6-1-3-1--4-3-2-1--1-=$ $9-1-6-4-6-1-2-1--4-3-2-1--10-$ $12-1-6-4-6-1-3-1--4-3-2-1--1-=$ $9-1-6-4-6---1-4-3-2-1=-1=$ $9-1-6-4-6-1-3-1--4-3-2-1--1=$ $6-1-6-4-6-2-2-1--4-3-3-1--1-$ $6-1-6-4-6----4-3-2-1--1=-$ $12-1-6-4-6-=-2-4-3-2-1=-1=$ $9-1-6-4-6-\ldots-4-3-2-1--1-$ $6-1-6-4-6-2-2-1=-4-3-3-1--1-$ $9-1-6-5-6--1--4-3-3-2--1-=$ $9-1-6-4-6-2-2-1-2-4-4-3-3--1=$ $14-1-8-5-8-2-2-1--4-4-1-3--1-$ $9-1-6-4-6----4-3-2-3--1=-$ $1-1-6-4-6-2-2-1=-4-3-2-2--10=$ $11-1-6-4-6-0--4-3--2--10=$ $9-1-6-4-6-2-2-1=-4-3-2-2--10=$ $12-1-6-4-6-2-3-1--4-3-3-2--1-$ $16-1-6-5-6-2-2-1-2-4-4-4-2--10-$ $1-1-6-6-8-2-2-1-2-4-3-3-2--10-$ $11-1-6-5-6-3-2-1--4-4-3-2--1=-$ $16-1-6-5-6-2-3-3--4-3-2-1--10=$ $9-1-8-6-8-2-3-1--4-4-2-1--1-$ $6-1-6-4-6-2-2-1--4-3-2-1--1=$ $9-1-6-5-6---1-4-3-2-1--10$
$6-1-8-6-8-2-2-1--4-3-2-3--1=$ $11---5-6----4-3-2-3--10-$ $11-1-6-4-6-2-2-1=-4-3-3-3--10=$ $6-1-6-5-6-\ldots-0-4-3-2-3--10=$ $11-1-6-4-6-2-2-1--4-3-2-3--1=$ $12-1-7-4-6-1-3-1=-4-3-2-2--10=$
$9-1-7-4-6--1-0-3-2-2-1-$

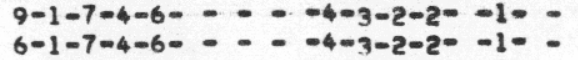
$6-1-7-5-6-1-3-1--4-3-3-2--9-$ -

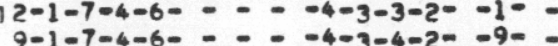

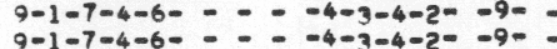
2-1-7-4-6- - - - -4-3-4-4-2- -9$6-1-7-4-6-:=-4-3-2-2--9-$
$9-1-7-4-6-=-4-3-2-2-=9-$ $9-1-6-4-6-\ldots-0-4-3-3-1--1-=$ $6-1-6-4-6-2-3-1--4-3-3-1--1-=$
$6-1-6-4-6-1---4-3-3-1--1-=$
5.90

3.20

3.50

3.50

3.20

3.30

3.10

3.00

3.50

3.60

3.30

3.60

2.80

2.60

$2.50^{\circ}$

2.70

1.60

1.90

2.70

2.50

3.70

3.40

2.60

1.60

2.100

2.90

1.30

2.20

2.70

3.60

$2.80^{\circ}$

2.30

2.90

3.70

3.90

4.00

3.90

3.10

3.30

3.70

3.50

5.20

3.70

3.80

3.20
2.70 
LASL Uranium Hydrogeochemical and Stream Sediment Reconnaissance Data Listing

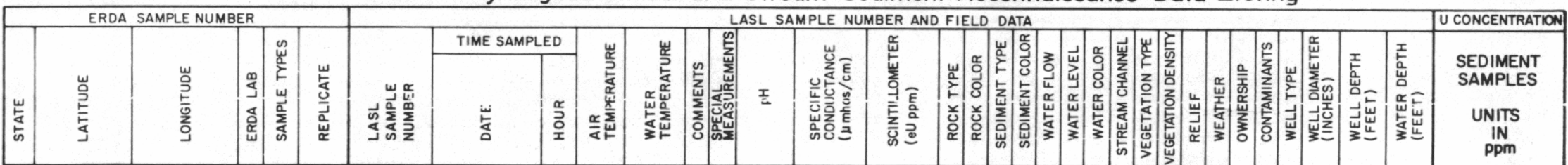

$50=44.8291-104.5727-?-15-$ $56=44.8286-106.5727-2-15-$ $56-44.0327-106.5338-2-14-$ $36-44$. $8655-104.5783-2-14-$ $36-44.1761-106.5023-2-15-$
$36-44.5980-106.3005-2-15-$ $36-44.5986-106.3505-2-15-$
$35-44 .>336-106.3519-?-15-$ $35=44.5836-106.3519-?-15-$
$56-44.5844-106.3500-2-15-$ $56-44.5844-106.3500-2-15-$
$56-44.6150-106.2477-?-14-$ $36-44$. $5116-104.2583-?-14$ $36-44.5033-106.27$ ग?-?-14$36-44.5325-106.2513-2-15-$ $36-44.0591-104.6477-?-11-$
$56=44.0561-106.675 ?-7-15-$ $56-44.0561-104.675 ?->-15-$
$56-44.0944-104.6980->-15-$ $56-44.1283-100.7394-?-15-$ $26=44.0744-104.7469-7-97$ $56-44.0441=100.71833-2-14$ $56=44.6366-106.7359-?-15$ $56=44.7106-106.6027-2-14$ $56-44.2294-106,4766-?-14=$ $56=44.2027=106.4927-?-97$ $36-44.1658-106.498 n=-14$ $56-44.1500-106.4755-2-15-$ $36-44.2880-106.4288-?-12-$ $56-42.7005-106.640 n-2-14$ $56-44.6483-106.8188-?-15$ $56-44.7052-104.755 n-?-15$ $56-44.1222-106.8025-2-14$ $56-44, y 744-106.8294-2-97$ $36-44,7694-106.7777-7-15-$ $36-44.9886-106.7697->-15$ $36-44, y 736-106.7411-?-15-$ $36-44.0967-106.7288-2-15-$ $36=44.8975=106.6544-2-96=$ b6-44.y $y 880-10 k .6733-?-15-$ $56-44$, y133-10R. $6338-2-14=$ $26=44.9338-107.0363-7-97=$ $56-44.9 n 36-107.083 n-7-12-$ $36-44, y 175-107.0958-2-10-$ $56=44.9301-107.0902-?-15$ $36=44.7416-107.0734-2-12=$ $56-44.3741-107.0=65-?-14=$ $56-44.5494-107.0325-7-14=$ $36-44.9733-107.0297-2-15-$ $56-43.61) 58-106.5583-5-15-$ $56-43.0055-10 \mathrm{~K} .5850-2-15=$ $56=43.5822-104.5694->-15-$ $56-43,3627-106.5663-2-15-$ $56-43.3947-106.1465-?-14-$ $56-43.5966-106.1758-2-15-$
1-20ก855-04/24/74-15- $15-$ $n-200856-n 4 / 74 / 7 h-15-15-$
$n-200857-n 4 / 74 / 7 h-15-18-20 . n=-0.9-340 n j-$ $n=? 00858-04 / 24 / 7 h-15-18-16.11-=-6.3-10 n 0-$ $-200859-14 / 24 / 7 h-16-18-$ $-200361-04 / 75 / 74-11-19-$ $->00862-n 4 / 75 / 75-12-2 n-$
$-2010863-n 4 / 75 / 74-12-20-$

1) -20 (1864-04/75/75-12- $20-16,0=-6.3-270-$ $n->03865-n 4 / 75 / 7 k-12=19-17 . n=-=6.3-270-0$ $-200865-04 / 25 / 74-13-18-1 \mathrm{~K} .0-C_{-}-6.9-4300-$ $->00867-04 / 75 / 76-13-18-$

-200869-n4/75/7h-14- 16-1n.n-C- - 6.h- 650$-200871-04 / 25 / 76-15-15-$ $->07875-04 / 75 / 75-15-17=$ $-200876-04 / 25 / 74-15-16-C_{-}-C_{-}-{ }_{-}$ $1000979-04 / 25 / 7 n-16-18-\quad-=-0.5=13000$ -?OกQBO-04/?5/7A-16-17-12.n-:-7.?- $1200=$ - $200982-n 5 / 33 / 74-11-16-13 \cdot n-2-6.1-2700-$

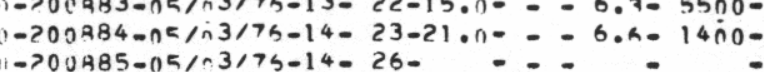
$\rightarrow>00 R 85-05 / 03 / 75-14-26-$

n-20ग926-n5/i3/74-15-22-11.n- - 6.9- $1250=$ $-200887-05 / 27 / 7 h-13-25-19.00-$ - 6.h- $8300-$ 1) $-200992-n 4 />9 / 7 h-1 n-7-\quad-C--\quad-=$ $n-2.00908-n 4 / 29 / 76-15-10-$
$11 \rightarrow 200910-04 / 29 / 74-15-10-7.0-0-6.9-65 n-$ $00918-04 / 30 / 7 h-11-17$ (1) $\rightarrow 00919-04 / 30 / 74-12-17=$ $n-200923-04 / 30 / 76-13-19-$ $n-200924-04 / 30 / 75-14-19-$ $1-200929=04 / 30 / 7 h-14-14-$ $1=200930-04 / 30 / 7 h-15-15$. $-200931-04 / 30 / 74-15-16-\quad:-:=$ $n-200934-04 / 30 / 74-14-17-16.0-0.1-21000-$

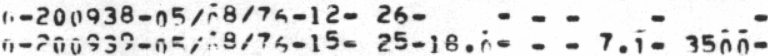
$-200940-05 / 11 / 76-10-17$

$1-200941-05111 / 74-10-17-\quad-=$ 1) $-200942-n 5 / 11 / 75-10-15-14 \cdot n-0-6.9-24 n 0-$ $11-200943-05 / 11 / 74-11-13-12 \cdot n--6=-3-120-$ $n=? 01944-05 / 11 / 76-12-15-15 \cdot n-C=-7 . ?-2550-$ 11-?0J945-0a/11/74-12-10$1=>00946-05 / 14 / 76-10-27=$
$-200947-05 / 14 / 76-10-27=$ $(1) 2019948-05 / 14 / 76-10-27-$

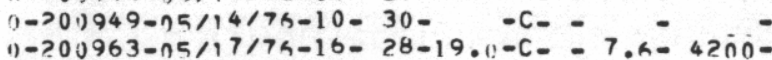
$n=>00964-05 / 17 / 75-16-25-$ - - -
6-1-6-4-6- - - - -4-3-3-1- -1- $6-1-6-4-6-1-2-8-3-3-1--1=$
$9-1-6-4-6-1-3-1=-4-3-3-1--1=$ $9-1-6-4-6-1-3-1--4-3-3-1--1-=$ $9-1-6-4-6-1-3-1--4-3-3-1=-1-$
$6-1-6-4-6----4-3-3-1--10$ $12-1-7-4-7----4-2-3-2--1=$

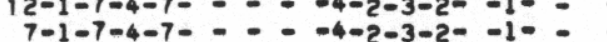
13-1-7-4-7- - - - -4-2-3-2- -1- $15-1-7-5-7-2-2-2--4-2-2-2--1--$ $6-1-7-5-7-2-2-2-1-4-2-2-2-1-1-$
$16-1-7-5-7-2-2-2-0-4-2-2-2--1-$ $9-1-7-4-7-0--4-2-3-2--1-=$ $9-1-7-5-7-2-2-1--4-2-3-3--1-0$

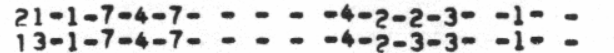
$12-1-7-4-7-0-0-4-2-2-3--10=$ $13-1-7-4-7-0-0-4-2-2-3--1-$ $1-1-i-5-7-2-3-2--4-8-3-3--1=-$
$11-1-7-4-7---1--4-2-3-3--1-$ $11-1-7-4-7--2-2-4-2-3-3--1=-$
$13-1-7-5-7-2-2-2--4-2-3-3--1=-$

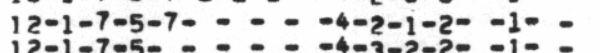
$16-1-7-5-7=0 .-0-3-2-2--1=$ $9-1-7-4-7-\ldots-4-3-3-3--1-=$ $11-1-7-1-1-1-1-3-3-2-3--1=$ $15-1-7-4-7-2-2-1--4-2-3-6--1=$ $12-1-7-4-7----4-2-2-3--1--$ $6-1-7-5-7-3-3-2--4-2-3-3--1-$
$9-1-6-4-6--$ $9-1-6-4-6-1-2-4-3-3-2--1=-$
$7-1-6-4-6-0-3-3-2-1-$ $12-1-6-5-6-\ldots-4-3-3-2--1=-$

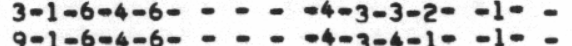

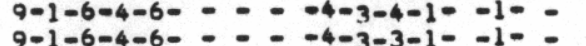
6-1-6-4-6- - - - -4-3-2-1- -1- $15-1-6-5-6-1-3-3--4-3-2-1--1-=$ $15-1-4-4-4-\ldots-4-3-3-1=-1=$ $1 \geq-1-4-6-8-2-3-1-2-4-3-2-1--1=-$ $9-1-7-5-6-=-4-3-3-3--1-$ $6-1-7-6-4--1-4-3-2-3--1=$
$6-1-7-5-6-3-3-3--4-3-2-3--1-$ $15-1-7-4-6-1-2-3--4-3-2-4--1-$ $15-1-7-5-6-1-3-1--4-3-4-4--9-$ -

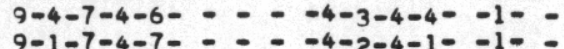

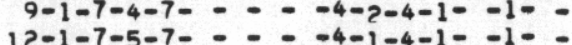
$12-1-7-5-7=--4-1-4-1--1=$

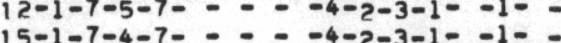
$9-1-7-5-7-2-3-1=-4-2-3-1=-1-$ 15-1-7-5-7-2-3-1- $-4-2-2-1--1-$
$15-1-5-7---1--4-7-2-1--1-$
2.50 3.10 3.30 3.00 2.90 3.20 3.90 4.10 3.40 8.70 6.00 4.30 2.70 2.50 3.70 $2.6 \bar{y}$ 2.80 30 2.90
4.00 3.70 3.70
4.40 4.20 3.60 9.30 3.20 5.50 3.00 3.40 3.90

2.60

2.90

3.20

3.60

4.30
3.70

4.40

4.90

4.20
4.60

3.60
3.30

6.70

3.30

3.50

3.50

5.60

5.60

5.50
4.20 
LASL Uranium Hydrogeochemical and Stream Sediment Reconnaissance Data Listing

$56-47.0222-106.1611-?-15-$ $35=43.6394-104.5455-2-15-$ $26=43.658 n-106.5255-2-15-$ $0.44 .0555-106.6355-2-12$ $56-44.0955-106.6365-2-97$ $06-44.0444-106.7208-2-14$ $56-4400133-106.725 n-2-15$ $56-44.1433-1060725 n=2-15-$ $56-44.1847-106.7138-7-140$ $56-44.1847-106.7138-7-14-$ $36-44.2502-106.68 n n-2-15-$ $56=44.0516-106.5951-2-12-$ $56-44.0333-106.7325=-15-$ $56-44.026 y-10 \mathrm{R} .703 \mathrm{~h}-2-97$ $36-44.2402-105 \cdot 54 B^{2}-2-13-$ $36-44,1 ? 61-106.7236-2-14=$ $36-44.0416-106.6555-2-14-$ $56-44.0283-10 k .6611-?-14-$ $56-44.1021-106.54 n n-2-14-$ $36-44.0622-106.5197-?-77-$ $36-44.0205-104.5297-2=97$ $56-44.0227-102.5033-2-15-$ $36-44.1$ ? $91-10 \% .6400-2-15-$ $36-44.0613-10 \% .5814-2-12-$ $56-44.0766-10 k .5588->-15=$ $56-44.1055-106.5697-2-14-$ $26=44.0388-106.4766-?-15-$ $56-44 \cdot 0444-10<\cdot 4144-2-14-$ $56-44.4233-106.7177-2-99-$ $56-43.2366-106.3227-2-15-$ $56-43.5155-1$ Uf. $3044-?-15-$ $56-43.5083-106.2833-7-15-$ $56-43.5177-106.5177-2-14=$ $36-43.5305-106.5377-2-14-$ $56-43.0271-106.320 n-2-15-$ $56-43.5980-106.2794-?-15-$ $56-43.595 i)-106.2083-2-15-$ $36-43.0 .141-106.2336-2-15-$ 36-43.5991-106.2211-?-15$56-43.7438-10 \% .6011-2-97=$ $36-43.7480-106.5469-2-15-$ $56-43.5952-106.1772-2-15-$ $56-42.5577-106.210 ?-7-14=$ $36-43,5744-10 h .225 ?->-15$ $36-43$.j505-10R.1877-?-14$36-43.5$ ? $11-10+.1744-?-14-$ $56-43$. $\left.255 ?-10^{k}, 14\right) 9-2-1 b-$ $36-43,0361-10 \mathrm{~h} .137 ?-2-15-$ $56-43 . j 525-106.1586-2-15-$ $56-43.570 j-10 k .155 n-?-15=$ $56-44.7555-106.9683-2-14-$
$11-200965-05 / 17174-16-27-$ $0=200967-05 / 17,76-17=29$

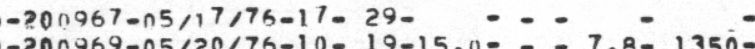
$n-20,1970-051>017 n-10-210$ - : -

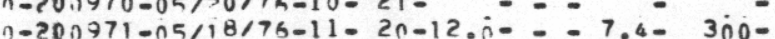
$-200972-05 / 18 / 74-12-25-17, n--7_{0} .6-30000-$

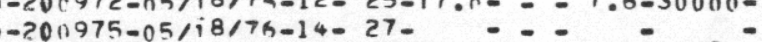

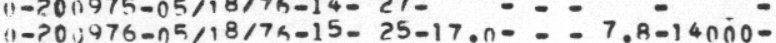
$0=200977-05 / 18 / 75-15-26-2 n, 0-0-7.8-10500-$

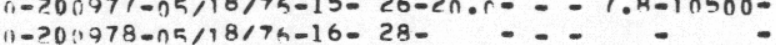
-2011980-05/18/7h-18-25-14.n- - - 8.?- $140^{\circ}$ $-200981-05 / 18 / 7 h-17-23-$ - : in $-200981-05 / 8 / 75-17-230$

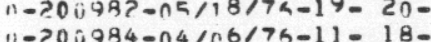

$n=? 0,0985-05 />0 / 74-10-20-16.0-=-8 . n-450-$ (1)?00986-05/30/75-12- $21-20 . n=-$ - 7.R- $8000-$ 11 $-20010987-0=2 / 20 / 7 k-12-20-2 n \cdot 1--7 \cdot 3-10000-$ - 3no" $=>011989-05 / 20 / 76-14-23-$ 1)>00990-05/20/7h-14- 2 n 1->n

$\rightarrow>0094-05 / 25 / 74-12-15-14010=-00-0$ $\rightarrow 00995-n 5 />2 / 74-13-19-\quad-c_{-}-6.3=310=$ $\rightarrow \rightarrow 0\{996-n 5 />2 / 74-14-19-19 . n-=-6.9-15 n 0-$

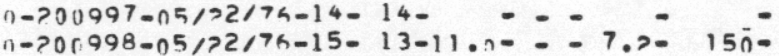

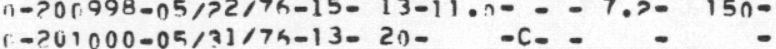
$n-501001-05 / 14 / 76-10-30=$ $n-? 0100 ?-02 / 14 / 7 a-16-31-$ $n=201003-05 / 14 / 74-16-3 n-\quad---$ $n=201004-05 / 14 / 76-17-28-21 \cdot n-0-7 \cdot 8-300-$ $n=201 n 06-05 / 14 / 7 n-17-27-19 . n-0-8 . n-16 n 0-$ $n=201007-05 / 16 / 75-13=20-$
$n=201008-09 / 16 / 79-14=20-$ $n \rightarrow>01099-05 / 16 / 7 a-14-20=$ $-201010-05 / 16 / 76-14-20-$ -ivilil-05/16/76-i4- 15$->01013-n 5 / 16 / 76-17-16-$ $-201014-05 / 16 / 7 h-17-16-\quad-C_{-}-:$
$\rightarrow 001015-05 / 17 / 76-12-27-$ $->01116-05 / 17 / 76-12-25-18.0-C--7.6-1700-$

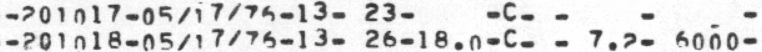
$-201019-05 / 17 / 7 h-13-25-18 \cdot n-C_{-}-8.4=200-$ $n-201720-05 / 17 / 7 n-14-25-$ 0
$-201023-05 / 17 / 75-15-28-$ $-201025-05 / 17 / 7 h-15-27-0-0$ $n-201030-04 / 30 / 76-14011-14 . n=-6.8-650-$

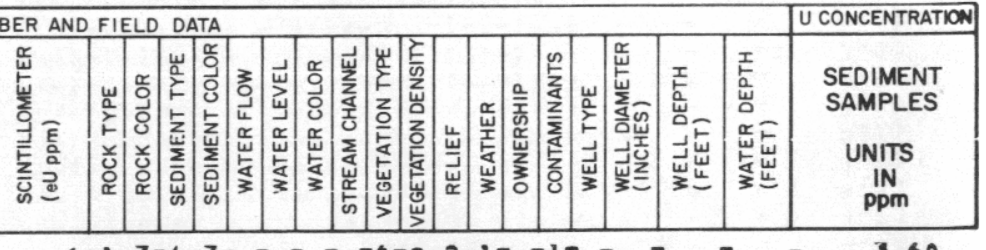

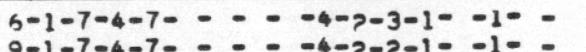
$9-1-7-4-7-\ldots--4-2-1-3--1-$ $3-1-7-5-6-2-3-=-4-3-2-2--1=$ 3-1-7-4-6-- - - -4-3-3-2- -1- $12-1-7-5-6-4-3-4--4-3-3-1--1-=$ $6-1-7-5-6-1-3-1--4-3-1-1--1=$ $9-1-7-5-6-1--4-3-4-1=-1-$ $12-1-7-5-6-1-3-2--4-3-1-1=-1=$ $1-1-7-4-7-1-3-2--4-3-1-1=-1-$ $9-1-7-4-6-4-3-1=-4-3-2-2--1-=$ $9-1-7-5-6----4-3-2-2--1=-$ $6-1-7-4-6-0--4-3-2-2--1=$ $-1-6-4-6--3--3-4-3-2-2--10=$ $6-1-7-5-6-1-3-3--4-3-3-2--1-$ $6-1-7-5-6-1-3-3--4-3-2-2--1-$ $6-7-7-5-6-1-3-3--4-3-2-2-1-1-=$ $6-1-7-5-6-1-3-3-4-4-2-2-2-$
$6-7-6-5-4-1-3-3--4-3-3-2--10$ $12-1-7-4-6---4-3-2-3--1=-$ $6-1-7=4-60=-0-4-3-2-3--1=$ $9-1-7-4-6-0-0-4-3-2-3--1-=$ $9-1-6-4-6-4-4-4-2-4-3-2-3--1-=$ $9-1-7-------4-2-2-3--1--$ $7-1-6-5-8--4-2--4-3-2-3--10-$ $9-1-6-4-6-\cdots--4-2-2-3--1-$

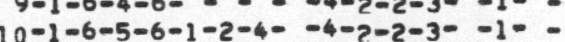
$6-1-7-5-6----4-3-2-5-1--$ $9-1-7-5-7-2-2-1=-4-2-3-1=-1-=$ $6-1-7-4-7----4-2-2-1--1-$ $9-1-7-4-7-\ldots-0-4-2-3-1=-1-$ $12-1-7-5-7-2-2-2-4-2-4-1--1=$ $15-1-7-5-7-1-2-2-04-2-4-10-1=$ $6-1-7-5-6---4-3-3-2-1-$ $9-1-7-4-6=-2=0$ $6-4-7-4-6-\cdots--4-3-3-2--9-$ $9-1-7-4-6-1=-8-3-3-2--9-1$ $9-1-7-4-6-1-2-4-3-3-2--9-$
$9-1-7-4-6-$ 6-1-7-4-6- - - - -4-3-2-1- -1- $6-1-7-4-7-\ldots--4-2-3-1--1-$ $9-1-7-5-7-1-2-2--4-2-3-1=-1=$ $9-1-7-4-7--7--4-2-3-1=-9-$
$9-1-7-5-7-2-3-1=-4-7-2-1=-90$ $9-1-7-5-6-1-2-2--4-2-3-1--9-$ $10-1-7-4-7-\div=-4-7-2-1--1=$ $12-1-7-4-7-\div=-4-2-2-1=-1-$
$9-1-7-4-7-\div-0-4-7-3-1=-1-$ $9-1-7-5-7-2-2-1--4-2-2-1--1-$ $10-1-6-6-8-2-2-1--4-3-2-2--1=$
3.60

3.80

3.70

5.90

3.00

3.90

3.10

1.10

$2,60^{2}$

3.00

8.80

2.80

3.0

4.4

3.00

3.10

3.20

. 00

3.10

.

2.40

(1)

3.00

1.8

1.8

0.0

3

3.0

3.50

3.2

3.0

3.30

4.20

2.80

$2.70^{\circ}$

30

3.00

3.10

$2.90^{\circ}$

3.20

3.10

2.90

3.10
3.30 
LASL Uranium Hydrogeochemical and Stream Sediment Reconnaissance Data Listing

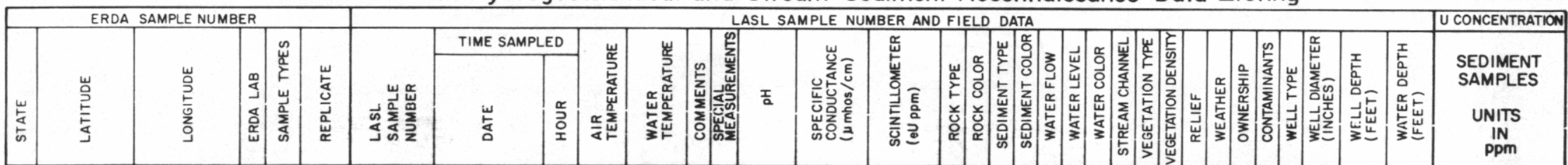

$56-44.1505-10 \mathrm{~K} .975 n-2-14-n-201332-04 / 30 / 7 n-15-13-15 \cdot 1-2-6.8-550-$ $06-44.0433-104.967 ?-2-13-$ $56-44.0527-10 t .895 n-2-15-$ $56-44.6708-106.9222-2-12-$ $36-44$. ngon-10t. $9466-2-14-$

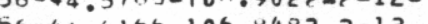
$56=44.5166-106.8483-2-12-$ $56-44.0147-100.8473-3-12-$ (36-4.040 $36-43 . \supset 915-106.7033-2-97=$

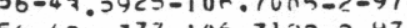
$56-47.0777-104.7123->-97-$ $36-43.5472-10 f .6783-2-97-$ $56-47.0672=104.5363-?-15-$ $56-42.0486-10+.5544-2-15-$ ग6-43.0466-104.6044-?-14$56-42.628 .1-10 f .6141-7-97-$ $36=43.70 y 4=10 \% .3544-\bar{c}-\mathrm{i} 5$ $56-43.7322-106.312 ?-?-14-$ $56-43.0873-106.2150-2-15-$ $56-43.088 n-104.2833-?-15-$ $36-43.0627-106.2706-7-14-$
$56-43.065 n-106.3055-7-15-$ $56-43.665 n-106.3055-?-15-$
$56-43.0607-106.3038-2-15-$
$56-43.0772-104.3250-5-97-$ $56-47.0775-104.3526-5-97-$
$56-43.0644-10 \% .3061-?-15-$ $56-43.0603-106.305 n-2-15-$ $56-43.1475-104.2583-2-15=$ $56-43.6488-10 \% \cdot 325 ?-?-97-$ $56-43.0522-10 \% .3127-7-14-$ $36-43.8008-104.4277-2-97-$ $36-43$. वा155-106. $4 / 38->-14-$ $56-43.7494-106.4488-2-91-$ $36-43.3458-104.396,6->-14-$ כ6-43.8722-10\%. $4294->-15-$ $36-43$. $0613-10+.6644-2-11$ $36-42.06111-10 \% .6627-2-14-$ $56-43.9494-106.71100-2-15-$ $56-43.8877-104.6606-2-15-$ $56-43.7472-10+.6844-2-14=$ $56-43.7725-104.6727-2-15-$
$56-43.7232-1116.0450-2-14-$ b6 $-43.7222-1$ 1) $.645 n-2-14-$ $36-47.7461-104.6016-7-14-$ 26-43.9444-10t. $385 n-2-15-$ $56-43$. $726 y-106.5377->-15-$ $56-43$. $9061-106$. $312 ?-2-97-$ $56-43$. 7h7 $2-10$ t. $5201-2-97$ ग6-47. $9921-10 \% .5750-?-15-$ $56-47.8891-106.5438-2-15-$ $n-201032-04 / 30 / 7 h-15-13-15 \cdot 1-=-6.8-55 n-$
$n-201034-04 / 30 / 74-16-14-12 . n-=-6.3-$ iño$->01036-04 / 30 / 7 \mathrm{~h}=17-16$

$-201 n 38-05 / 11 / 7 h-12-14-12.0-0-6.8-950-$ - $01 n 4 n-05 / n 17 h-13-2 n-12 . n=-6.5-550$ - $20104 B-05,4175-170-14-10.0=-6.5-100$ $->01049-05 / n 1 / 7 h-18-14-10.0=-6.8-550-$ - $01050-05 / n 17 n-18-14-10011=-6.8-700$

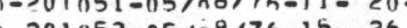
$->01053-05 / 28 / 76-15-26-$ $\rightarrow 01055-05 / 1,8 / 7 a-16-26-$ $->01057-05 / i 8 / 75-18-23-$

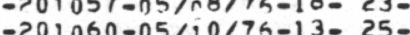
( $-? 01062-05 / 10176-14-24-18.5-=-6.5-11250=$ ->01063-05/10/7h-17- $18-$

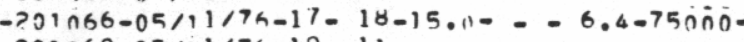
(1) $\rightarrow 01071-05,12174-12-14-15, n-C_{-}=6.4-3850-$ $->01073-05 / 12174-16-11-$ $->01976-05 / 12175-10-18-$ $-201977-05 / 12 / 75-18-17$ $\rightarrow 01078-05 / 13 / 74-11-19$ $-2-10$ -? 01 ก $80-05 / 13 / 76-12-25-23.5=-=6.5=36 n 0=$ $->01083-05 / 13 / 7 k-18-23$ -?01084-05/14/74-11-24-17.5-- - 6.5- $210=$ $->01085-05 / 13 / 7 n-18-21-$ -201086-05/14/74-12-25-21.5-- - 6.5- $220-$ - $201087-05 / 14 / 74=140-58-0$

-201 n88-0a/14/7h-15-24-12.11-C- - 6.7- 15no$n=>01089-05 / 14 / 7 h-16-23-21.5--0.6-1550-$ $-20109 ?-05 / 17 / 7 h-11-24-$ $-2 n 1 n 94-n 5 / 18 / 7 h-11$. ? $n=2 n 1 n 98-n 5 / 18 / 76-18-23-20.0=-=7.5-1625-$ $1 \rightarrow 001100-n 5 / 19 / 7 n-10-18-17, n=-0.3-7 n 0=$ $n-201101-05 / 19 / 7 n-11-19-17 . n-0-8.5-165$ $1 \rightarrow-701102-05 /, 9 / 74-11-20-$ $n-201103-05 / 19 / 76-12-23-$ $-201105-05 / 19 / 76-15-24$ $\rightarrow 01108-n 5 / 19 / 7 h-15-25-$ - $201110-05 / 19 / 7 a-16-24-25 \cdot 0-=-6 \cdot R-700-$ n->01111-05/>0176-10-19- … $---2=$ $-201075-05 / 12174-17-18$ - $201107-n 5 / 19 / 7 h-15-23-$
$11-1-6-4-8-3-2-1--4-3-2-2--1=$ $2-1-6-4-6-2-2-1--4-3-2-2--10$
$6-1-6---1--1--4-3-2-1--10$ $16-1-6-4-6-2-2-1=-4-3-2-1--1-$ $3-1-6-3-6-3-2-1=-4-3-2-1=-1-$ $14-1-6-4-6-3-4-1=-4-3-5-1=-10$ $12-1-6-4-6-2-2-1--4-3-2-1=-1-$ $1>-1-6-4-6-2-2-1--4-3-2-1=-10$ $9-1-1-5-7-1-3-3-4-3-2-1--10$ $14-1-1-5-6-0--4-3-1-1=-10$ $16-1-7-5-6-2-2-3-1-4-2-2-1-=1 \alpha$ $9-1-1-5-6-\cdots-4-2-1-1=-10$ $14-1-1-5-6-\ldots-4-3-2-1-$ $9-1-1-5-1=--4-3-2-2--1-$

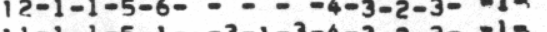
$11-1-1-5-1--3-1-3-4-3-2-3--1 a=$ $21-1-1-5-7-1-2-2-3-4-4-5-4--1 \times$
$19-1-1-5-1-1-1-1--4-2-3-4--1 a$ $19-1-1-5-1--1-1-2-4-2-3-4--1<=$ $14-1-1-5-7-2-3-1-2-4-3-2-2--1=$ $11-1-4-4-1-:-2-4-3-2-2-$
$9-1-4-10$

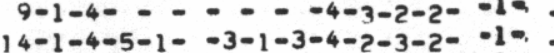
$14-1-4-5-1--3-1-3-4-2-3-2--1-$
$14-1-1-4-6--1--4-4-1-4-2--1$ $9-1-4-4-6--1--4-1-4-2--1=$ $19-1-1-5-7--1-1--4-3-1-2-010$
$12-1-1-5-1-1-2-1-2-4-2-3-2--10$ $12-1-1-5-1-1-2-1-2-4-2-3-2--1=$
$9-1-1-5-1-1-1--2-4-2-3-2--10=$ $9-1-1-5-1-1-1--2-4-2-3-2--1=$
$16-1-1-5-1-1--4-3-2-1-1=$ $12-1-1-5-6--1--4-3-3-1=-1=$ $12-1-1-5-7--2-4--4-7-3-1=-1=$ $11-1-6-5-6---2-4-3-1-1=-1=$ $11-1-6-5-7-1-3-1-3-4-3-2-1=-1=$ $11-1-6-5-6--1-0-4-3-1-1=-1=$ $10-1-1-5-7--2-1-4-4-3-2-1=-1=$ $15-1-1-5-8-2-3-1-3-4-3-3-2--1-$ $11-1-1-5-8-2-3-1-3-4-3-3-2--1 a$ $12-1-1-6-7-3-4-3-3-4-2-3-1--1-$ $12-1-1-4-1--1--4-3-3-1--1-$
$15-1-1--1-3-1--4-3-2-2--1-$ $15-1-1--1-3-1--4-3-2-2--1=$ $6-1-1-5-7-3-3-1-1-4-3-2-2--10$
$1-1-7-5-7-1-3-3--4-3-2-1--10$ $11-1-7-5-7-1-3-3--4-3-2-1=-1=$ $8-1-7-5-8-1-3-3--4-3-2-1--1=$
$1-1-7-5-6-1--0-4-3-3-1--10$ $11-1-7-5-6-1-0-4-3-3-1=-10$
$13-1-3-5-6-0$ $17-1-7-5-6=-0-4-3-2-1=-1=$ $11-1-7-5-6-\cdots-4-3-1-1=-1$ 13-1-7-5-6- - - -4-3-3-1- -10 $11-1-7-5-6--4-3-2-1--1-$
$5-1-7-5-8-1-3-3--4-3-3-2--1-$ i $5-1-1-5-6-1-1--2-4-3-2-2--1=$
3.10 1.70 1.70 2.80 50 2.80 2.30 4.50 4.10 3.40 3.00 4.50
3.20 .40 .00 4.10
3.70 $2.8 \mathrm{~g}$ 6.20 3.80 3.40 2.90 3.00 $4.30^{\circ}$ 4.10 5.50 3.50
4.90 2.80 2.70 2.90 2.60 2.60 4.80 3.80 2.70 2.60
3.10 3.20 3.70 3.90 3.60 3.50 3.60 3.70 3.30 3.20 3.10
2.90 
LASL Uranium Hydrogeochemical and Stream Sediment Reconnaissance Data Listing

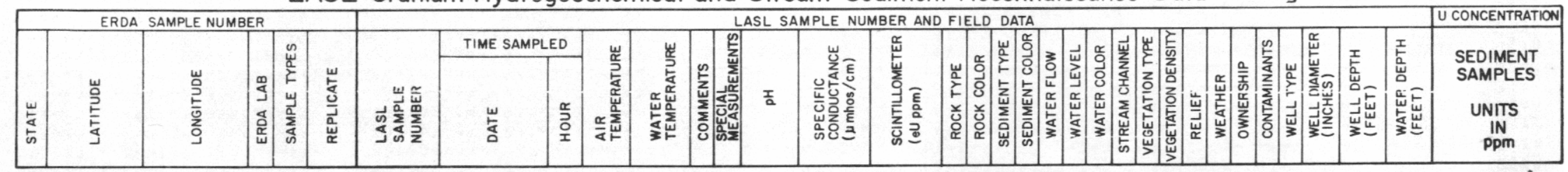

$36-43.8638-10+.546 n-7-14-$ $56-47.89511-10 \mathrm{H} .5566-2-97$

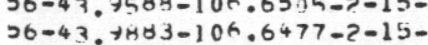
$56-43$. $403-105.6477-2-150$ $06-4.2491-105.5966-2-97=$ $56-44$. ग $35-10$. $56-44$. ग76 - 0 की. $36-44$. 758 s -10 r. $9605-2-12=$ $56-43.5455-104.5338-7-15=$ $56-43.5455-106.538=2-15$ $36-42 .>444-106.5344-2-15$ $56-43.5844-106.5061-2-14-$

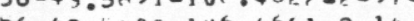
6-43. b40 $-106.4661-2-14=$ $56-43.5005-106.4488-2-97$ $56-43.5216-106.4244-2-15$ $56=43.53: 0=10^{2} \cdot 420 n-2-14-$ $36-4 ? .5480-1$ UR. $4086-2-15-$ $56-43.5666-106.389 n-2-15$ $56-43.5644-106.3588-2-15-$
$56-43.5100-106.3583-?-97$ $56-43.5438-10+.3288-2-15-$ $36-44 \cdot 7455-106.9311-?-14-$ $36-43.7697-106.6052-2-97=$ $56-43.8175-106.5738-?-97-$
$36-43.8269-106.6116-2-97-$ $36-43.8516-106.6065-2-97=$ $36-43.8594-106.7033-2-97=$ $26-43.7233-10^{4} .5516-2-15-$ $56-43.6994-10 t .5338-2-15$ $36-43.6022-106.5150-2-15-$
$56-43.7597-106.4850-2-15=$ $26-43.8452-10 \% .5291-2-97=$ $36-43.8641-10 \% .5083-2-97$ $56-43.7069-106.3866-2-97-$
$56-43.7233-106.42111-2-97-$ $56-43.7233-10 t .4211-2-97-$ $56-43.6980-106.4497-2-15-$
$26-43.6944-106.4075-?-15-$ $26-43.6944-106.4675-2-15-$ $36-43.7202-106.6433-2-15=$
$56-43.7350-106.6869-2-97$ $56-43.1702-106.7080-7-12$ $36-43.7950-10 \mathrm{f} .7227-2-97=$ $56-43.6572-106.7294-2-15-$ $36-47.0486-106.7488-2-97=$ $56-43.6655-106.7044-2-97$ $56-43.6622-10+.6927-2-97=$ $36=43.6475-10 \mathrm{~K} .700 \mathrm{~B}-2-2-97-$ $56-43.6838-106.743 \mathrm{~B}-\mathrm{-2}-15-$ $56-43.6961-10 \mathrm{H} .7269-2-13-$ $36-43.7186-104.7298-2-97-$ $56-43.6316-104.6338-2-15$ - a->n1113-05/20/7n-11-19-19.5-- - 6.3- 20i5$11-201115-05 / 20 / 7 h-12-22-$ $\rightarrow 0117-05 / 21175-11-2 n-$ $-201126-04 / 7 \pi 7 / 75-12-18$ $n-201128-05 / n 717 k-13-2 n=$

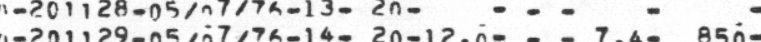
$0-291130-05 / 17175-15-16-1200-7.1-700-$ $n->01132-05 / n 7 / 7 n-16-2 n-18,0=-7,5-40 n 0-$ $-301134-05 / 14 / 74-11-28$

$1-201135-n 5 / i 4 / 7 h-11-28-\quad-0_{-}-$ $-201137-05 / 14 / 7 h-12-33-21 \cdot 1--7_{0} \cdot 8-34 n 0-$ $-201139-05 / 14 / 74-13-31-20.10=-8.2-15 n 00$ $\rightarrow->01140-n a / 14 / 74-13-32-$

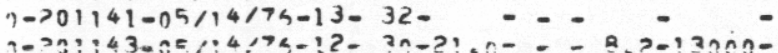
$501144-05114175-15035$ $\rightarrow 001146-05 / 14 / 79-14-31-$ $n-201147-05 / 14 / 7 n-15-31-$ त->्रा149-nas - - - - - - -

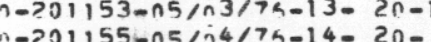
n-PC $157-05 / 14 / 75-14-24$ $\rightarrow 01158-05 / 34176-15=25$ $n-201159-05 / 74 / 74-15-22-$ $1 \rightarrow 01161-05 / n 4 / 75-16-21$ $3-201162-05 / 15 / 76-9-15$ $n=201164-05 / n 5 / 76-10-13-$ $n=201165-05 / 25 / 7 a-10=13-$
$n \rightarrow 201166-05 /$ ins/7n-11: $14-$ $n=201168-05 / 25 / 76-13-12-$ - $201169-05 / n 5 / 7 b-14=15-$ $n \rightarrow 01171-05 / n 5 / 7 h-15-15-$ $0-201173-05 / 75 / 76-16-17=$ $n-201175-05 / 25 / 7 a-17-2 n-$ $0-201176-0,5,-5 / 75-18-17-$ $n-201177-n G / n 5 / 74-8-1 n-$ $n=201178-04 / 75 / 75-9-14-$ - - - $1->01181-05 / n 6 / 74-10-13-$ $n-20118>-n 5 / i 6 / 7 h-13-16$ $n \rightarrow ? 01183-n 5 / 46 / 74-13-18=$ $n-201184-n 5 / i 6 / 7 n-14-17$ 11-?01185-05/n6/74-15- $17=$ $1=? 01186-05 / 106 / 76-15-17=$ $n=201187=02 / n 6 / 76-16-15-$ $n-201189-05 / n 6 / 74-16-15$ $n-? 01190-05 / 26 / 75-17-13-$
$n \rightarrow 001191-05 / i 7 / 74-12-18-$
$11-1-1-4-7-1-2-2-3-4-3-2-2--1=-$

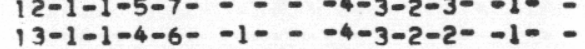
$14-1-1-5-6--1---4-3-2-2--1=$ $3-1-1-5-8--1=-4-3-3-1--1=$ $6-1-1-6-8-4-3-4-2-6-3-3-1=-1=$ $6-1-6-5-8-3-3-4-2-4-3-2-1--1=$ $6-1-6-5-8-3-3-4-2-4-3-2-1=-10$ $10-1-6-5-7-1-3-1--4-4-2-1=-1:$
$13-1-7-4-7-1-1-$ $18-1-7-4-7-0--4-2-3-1--1=$ $15-1-7-5-7-3-3-1=-4-7-2-1=-10$ $12-1-7-4-7--1--4-2-2-1=-1-$ $6-1-7-5-7-2-2-1--4-2-2-1--10$ $15-1-7-5-7-\cdots-4-2-2-1--1=$

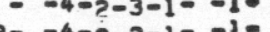
$9-1-7-5-7-2-2-2--40-2-1=-10$ $12-1-7-4-7-1-0-2-3-1=-1-$ $9-1-7-4-7--1--4-2-3-1=-1-$ $12-1-7-5-7-3-3-1=-4-2-3-1=-10$
$12-1-7-5-7--0-0-2-3-1=-10$ $6-1-7-5-7-2-2-1--4-2-3-1--1$ $19-1-8-6-8-2-2-1--4-3-2-2--1-$ $6-1-7-4-6----4-3-3-2--10$ $12-1-7-5-6-=--4-2-2-2=-10$ $12-1-1-4-6-1--4-2-2-2-11=$ $9-1-1-5-6-2-0-4-3-2-2=-10$ 6-1-1-5-6- - - -4-3-2-2- -1-

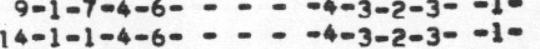
$14-1-1-4-6-6=-1-4-3-2-3-10$
$21-1-1-1-6-2=-10$ $9-1-2-5-2-=-0-4-3-2-3--10$ $6-1-1-5-6-1=-4-3-1-3--10$
$16-1-1-5-6-0-4-3-2-3--10$ $14-1-1-4-6-\ldots-0-4-3-2-3--10$ $14-1-1-4-6-1-0-4-3-2-3-10$
$12-1-1-5-6-10$

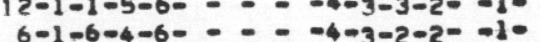
$6-1-6-4-6-0=-4-3-2-2--10$
$6-1-6-4-6-0$ $4-1-1-5-7-\ldots-4-2-2-2--10$ $14-1-7-5-7-\cdots--4-3-3-2--1-$ $10-1-7-5-7-2-2-2-2-4-2-2-3--1=$
$3-1-2-5-6-2-0-4-3-2-2--10$ $16-1-7-5-8-\ldots-4-7-3-3--1=$ $14-1-7-5-8-=-1-4-1-4-3--1=$
$8-1-7-5-7=-0-4-3-3-3--11$ $8-1-7-5-70=-1-4-2-3-3--1$
$16-1-7-4-7-0-10$

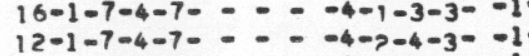

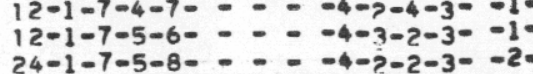
$24-1-7-5-8-2-4-2-2-3--2$

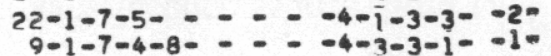

3.60

5.80

0.0

2.70

2.70

3.

5.00

3.40

30

3.30

3.3

3.70

2.60

30

2.90

3.30
2.70

3.6

3.60

3.7

3.70

(3.0

3.30

3.20

2.90

2.80

4.00
3.30

3.50

28

2.80

3.60

4.60

3.80

3.30

4.80

3.60

.5

4.70

5.00

4.50

4.00

3.70

5.60 
LASL Uranium Hydrogeochemical and Stream Sediment Reconnaissance Data Listing

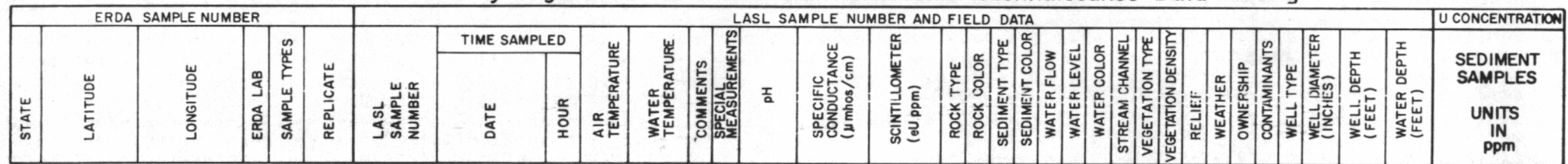

$56-43.0169-104.6438-2-15-$ $26-43.515,106,106.7086-2-12-$ $36-43.6766-104.6283-2-14-$ $36-43.6766-104.6283-2-14-$
$56-43.6916-106.5994-2-14-$ $56-43.6916-106.5994-2-14-$
$56-43.0961-106.6227-2-97-$ $56-43.0961-106.6227-2-97-$
$56-43.6344-106.6019-2-12-$ $56-43.6344-106.6019-2-12-$
$36-44.4333-106.7033-2-99-$ $36-44.4333-106.7033-2-99-$
$56-44.2077-106.3436-2-14-$ $56-44.2077-106.3436-2-14-$
$36-44.21111-106.37111-2-15-$ $36-44.2111-106.31111-2-15-$
$56-44,230 n-106.3716-2-15-$ $36-44.1469-106.3036-2-97-$ $56-44.1894-104.3019-?-15-$ $36-44.1797-104.2911-2-15-$ $56-44.1750-106.2755-2-14-$ $56-44.1688-106.2727-2-15-$ $56-44.2005-106.2938-2-14-$ $56-44.2461-106.3072-2-97=$ $56-44.2805-106.2702-2-15-$ 56-44.275n-1 Ut. $3194-?-14-$ $56-44.3472-106.2583-2-15-$ $56-44$. 3866-106. $4666-?-15-$ $36-44.4252-10 t .4591-2-97-$ $36-44,4447-106.4 \geq 44-2-97=$ $56-44.4161-106.4186-2-14-$
$56-44.4038-106.4075-2-97=$ $36-44,415 n-106.3794-2-14-$ $56-44.4269-106.3755->-14-$ $56-44.0972-105.2583->-97$. $36-44,1097-105.3369-2-15-$ $56-44,1205-105 \cdot 325 A-2-15-$ $56-44.1291-105.4041-2-15-$ $36-44,1644-105.4455-2-15-$ $36-44.2327-105.38 n 5-2-15-$ $56-44,23 \cup n-105.4347-2-15-$ $36-44.0994-105.0691-2-97-$ $56-44.0755-105.0555-2-97=$ $56-44.0936-105.1788-2-15$ $36-44.4711-105.8977-7-15$ $26-44,4485-105.8661-2-15-$ $56-44.4547-105.8583-2-15-$ $36-44.4666-105.7766-2-15-$ $56-44,4416-10<.7569-7-15-$ $56-44,4 ? 25-105.8602-2-1 b-$ $56-44 .+061-105.8286-2-15-$ $36=44.6658-105.0661-7-15-$ $56-44.050 n-105.1055-2-15-$ $56-44,5241-105.170 ?-2-15-$ $56-44.54 y 7-105.2013-?-15-$ $56-44 \cdot 1463-105.1361-2-14-$
$36-44,1430-105.1255-2-15-$ $n=201$ i92-05/i7/76-15- 18

$-\mathrm{C}_{-}-$ $n=201194-05 / n 7 / 76-18-20-21.5--6.9-315000-$ (17-14.n- - - 6.4-245001
$0-201198-05 / 198 / 76-9-19-11.5-c_{-}-6.5-37500-$ $n-201198-05 / 38 / 76-9-19-11.5-C_{-}-6.5-38 n 00-$
$n-201199-05 / i 8 / 76-9-17-\quad-C_{-}-a_{-}$ n-201?00-05/18/7a-11-19-19.n-- - 6.8-330000$->01202-05 / 31 / 7 h-14-18-$ $n \rightarrow 001>11-05 / 31 / 75-12-26-22 . n-0-6.3-250-$ 10

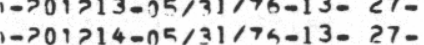
$\rightarrow 01515-n 5 / 31 / 74-13-57-$

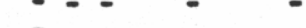
n->01>17-na/21/74-13-29-23.n-C- - 6.n- 9no-

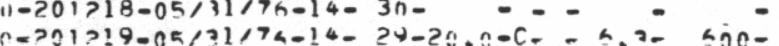

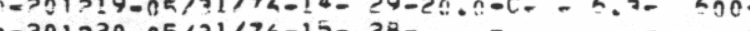
n- $01720005 / 317$ hat $n=501222-05 / 31 / 7 n-15-30-25, i=-E .3-30 n 0-$ $-201>23-02 / 31 / 7 n-16-27$ (1) $-201>28-n k / n 1 / 7 h-13-24$

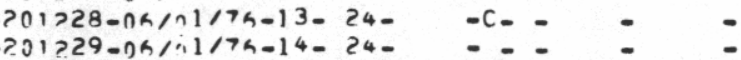
$1=>01>30-n k / 11 / 7 k-15-25-19.11--2.3-21 n \pi-$

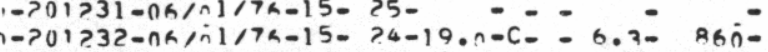
$n->01233-06 / 91 / 7 a-15-25-2 n \cdot n--6.3-2300-$ $-201>35-n 7 / 23 / 7 k-11-26-$ $1:->01237-07 / 23 / 76-12-311-$ $n=? 01>38-n>/>3 / 7 n-12-22-$

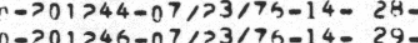
月- $-501>49-07 / 33 / 75-15-27-$ $->01314-07 />3 / 7 h-15-32-$ - $-201317-07 / 24 / 7 k-15-29-$ $-201320-07 / 34 / 7 a-15-29-$ $-201322-n 7 / 34 / 75-17-23-$ $-201328-1 n / 18 / 7 h-11-19-$ - $0133 n-16 / 98 / 74=11-19-$ - $01334-11 / n 8 / 75-12-20-$

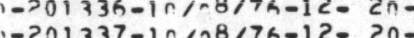
年 $-201340-07 />4 / 75-11-35$ $1->01342-07 / 74 / 76-12-35$ $1->01347-n 7 / 74 / 76-14-36-$ i) $-? 01355-n 7 / 24 / 7 k-17-35$

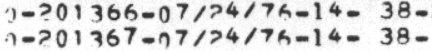

$\left\{\begin{array}{l}13-1-7-4-8----4-4-2-3-1--1=- \\ 12-1-7-4-7-3-3-1-2-4-2-3-1--1-\end{array}\right.$ $2-1-7-4-7-3-3-1-2-4-2-3-1--1=-$
$9-1-7-5-6-1-1-2-4-7-1-1--1-$

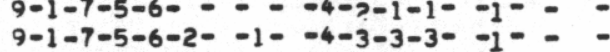
$11-1-6-5-6-1-2-1--4-3-3-1--1-=$ $14-1-6-4-6----4-3-2-1--1--$ $11-1-7-4-6-2-3-1-2-4-3-3-1--1-=$ $3-1-7-4-6----4-3-2-3--10$ $6-1-6-5-6-1-2-2--4-2-3-1--1$ 15-1-7-4-7--1-- -4-2-3-1- -1- $16-1-7-4-6--1---4-7-3-1--1-$ $12-1-7-4-6--1=-4-2-3-1--1-$
$12-1-6-4-6--1-=-4-2-3-1--1-$ $10-1-6-4-6--1--4-2-3-1--1-$ $7-1-6-5-7-1-2-2--4-2-2-1--1-$
$9-1-6-4-6--1--4-2-3-1--1=$ $1>-1-6-5-6-1-2-2--4-2-3-1--1=$

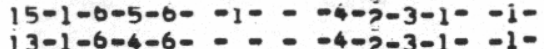
$10-1-6-5-6-1-2-2--4-2-3-1=-1-$ $13-1-6-4-6--1--4-2-3-1--1$
$15-1-6-4-6--1=-4-2-2-1--1$ $15-1-6-4-6--1=-4-2-2-1=-1$ $10-1-6-4-6--1--4-2-2-1--1$
$10-1-6-4-4--1-$ $13-1-6-\cdots-\ldots-3-3-2-2--1=$ $9-1-6-4-6--1-1=-4-2-2-1=-1-$
$9-1-6-5-6-2-3-1=-4-2-2-1=-1-$ $15-1-6-5-6-2-2-2--4-2-2-1=-1=$ $3-1-7-5-6-\cdots--4-3-2-1--1$ $9-1-6-5-6-1-2-4-3-2-1--1-$
$3-1-6-5-6-1-$ 15-1-7-5-6- - - -4-3-2-1- -10 $4-1-6-5-6-1-0-4-3-1-1--1-$
$9-1-7-5-6-0-3-3-2-1-1-$ $9-1-7-5-6-=-3-3-2-1=-1-$ $1-1-6-5-6-=-4-3-2-1--1=$ $1-1-1-4-6-=-4-3-2-1=-1=$

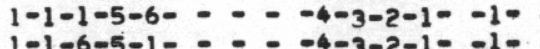
$11-1-1-4-6--1=-\bar{c}-4-3-2-1=-i=$ $16-1-7-4-6--1--2-2-1-2-1--1-$ $14-1-7-5-6--1--2-4-3-2-1=-1-$ $15-1-7-5-6--1--2-4-3-2-1--1=$ $11-1-7-4-6--1=-2-3-3-2-1=-1-$ $9-1-7-5-6--1=-2-3-3-2-1=-1-$ $6-1-7-4-6---2-3-2-1=-1$ $11-1-7-4-6-\div--4-4-2-2-1--1=$ $10-1-7-4-6----2-2-2-1--1-$ $12-1-6-5-6-2-2-2--4-2-2-1=-1=$
$7-1-6-4-6-5-1=-4-2-2-1--1=$
6.5

3.90

3.60

3.7

3.30

4.20

2.20

3.30

4.30

$3.50^{\circ}$

4.49
1.90

3.70

3.70

3.60

4.30
2.90

.70

4.00
3.80

.10

2.70

2.20

2.90
2.90

3.40

3.10

3.20

4.20

5.10

4.10
3.90

.60

3.70

2.90

3.60

.80

.30

3.00

.40

3.90

4.50
3.90

7

2.80

2.50

3.30

2.90

3.50
3.40 
LASL Uranium Hydrogeochemical and Stream Sediment Reconnaissance Data Listing

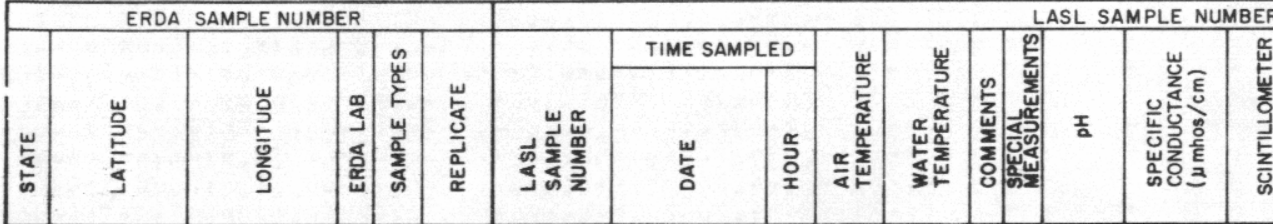

$56-44,1330-105.1616-2-15-$ $56-44.1527-105.1847-2-97-$ $56-44.1722-105.1311-2-97-$ $56-44.0761-105.1369-2-15-$ $56-44.0272-105.1547-2-15-$ $56-44.0650-105.1627-2-14-$ $56-44.0505-105 \cdot 173 n-2-15-$ $56-4.0406010500-2-15-$ $56-44.0166-105.0294-2-15-$

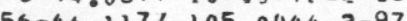
$56-44.1171-105.0044-2-97-$ $36-43.6652-107.009 n-2=14-$ $36-43.6522-107.0355-2-13-$ $56-43.0444-107.053=-2-11-$ $36-43.0594-107.0544-2-11-$ $36-43.6630-107.068 n-2-12-$ $56=4 ? .6647-107.0588-2-12-$ $50-43.6877-10701211-2-11-$ 56-4.6661-10 $7.1094-2-11=$ $36-43.6766-107.0827-2-11-$ 36-43.0544-107.0672-2-11$36-43.0280-107.0588-2-11=$ $56-43.6100-107.0666-2-11-$ $36-43.6222-107.0627-2-11-$ $56-43.6352-107.1151-2-11-$ $56-43.6352-107.1150-2-11-$
$56-43.6722-107.02 n 0-2-14-$ $56-43.6722-107.02 n c-2-14-$
$56-43.6788-107.0338-2-11-$ 56-43.7055-106.7777-2-14$56-43,700 n-106,7763-2-11-$ $56-43.7388-10 \% .7791-2-14-$ $56-43.0801-106.7638-2-97-$ $56-43.6888-106.7791-2-14-$ $56-43.6958-106.7716-2-11=$
$56-43.6458-106.7861-2-114=$ $56-43.6055-10 \% .8122-2-15-$ $56-43.5958-106.8>27-2-11$ $56-43.5875-106.8416-2-11$ 56-43.5708-106.8375-2-14$56-43.5541-106.8041-2-11-$ $56-43.5527-106.7930-2-11-$
$56-43.5472-106.8041-2-11-$ $56-43.5472-106.8041-2-11-$ $56-43.5888-106.8888-2-15-$
$56-43.5722-106.8944-2-15-$ $56-43.5722-106.8744-2-15-$ $56-43.5611-106.8708-7-15-$
$56-43.6208-106.9227-2-11-$ $56-43.6208-104.9227-2-11-$ $56-43.7133-107.0111-2-97=$ $56-43.7161-107.0283-2-97-$ $56-43 \cdot 7177-107 \cdot 0255-2-11=$
$56-43,7111-107,0527-2-14-$

$-201368-07 / 24 / 76-14-37-$ $-201369-07 / 24 / 75-14=36$ $->01370-n 7 / 24 / 76-15=35$ $-201372-n 7 / 26 / 7 a-12-31$

$\therefore:-$ (1) 201375-07/26/79-13-32-25.n- - 6.4- 43 ino $-201376-07 / 26 / 76-13-32$ .

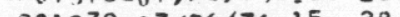
(1001383-07/26/7a-16= $-201451-07 / 048 / 76-10=27-20,0=-7.4-400-$ $-? 01452-n 7 / i 4 / 74-1 n-27-19.0---7.2-2 n 0-$ - $201454-07710474-10-28-10.11--7.4-250-$ 作 $\rightarrow 01457-07 / 24 / 7 n-11-28-11$.il- - - 6.R- 300

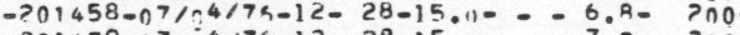
ח ->01460-07/144/74-12-28-19.0- - 7.?- $150-$

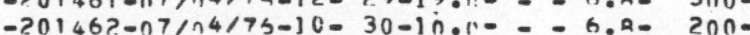
$-201464-07 / 74 / 74-14-31-11 \cdot n=--6 \cdot 8-450-$ $-201466-n 7 / 24 / 76-15-31-1 n \cdot 1)=-$ - 6.2- $2 n 0-$ 1, $-701469-n 7 / n_{4} / 7 h-18-32-13.0=-6.3-350=$

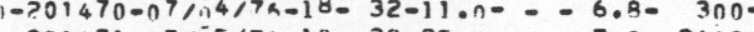
$n-201471-07 / C 5 / 7 h-10-28-25 . n=-7_{0} \cdot R-2600-$

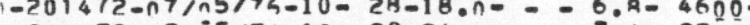

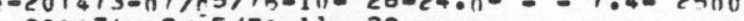
$1=? 01475-07 / n 5 / 7 h-11-29-25.0=-6.8-1100=$ $n=201476-07 / 05 / 74-11-29-19 \cdot 0=-0.80-500=$
$n-201477-07 / 15 / 76-12-30-25.00=-8.7-2000-$ $->01478-07 / 25 / 7 a-13-32-$

$->01479-07 / 155 / 75-13-32-25.0=-7.4=850$

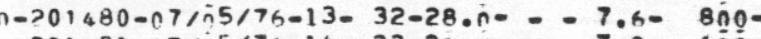

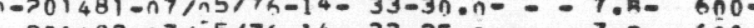

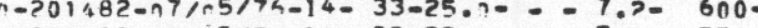
$->01483-07 / 75 / 73-14-33-23 . n=--7.4-550-$ - $201484-07 / n 5 / 74-14-35-22 . n-=-7.2-500$ $n-201485-07 / 25 / 76-15-35$

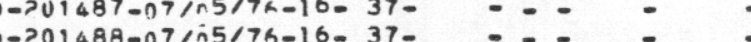
$\rightarrow 001489-n>/ n 5 / 7 h-10-35-19 . n=-0.8-300-$ $n=>01491-07 / 1,8 / 7 k-10=30-$

$-201493-07188 / 75-10=30-1100=-0.0-550$ $-201493-n 7 / n 8 / 7 a-10-30-11 \cdot n=-0.9-55 n-$
$c \rightarrow 201494-07 / 188 / 76-10-30-23.00=-6.5-200-$

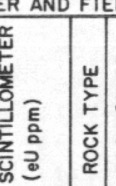

$12-1-6-4-6--0-3-3-2-1--1-=$ $7-1-6-4-6--1=--4-2-2-1--10$
$10-1-6-4-6--1-$ $10-1-6-4-6--1=--3-3-2-1--1-$
$10-1-6-4-8--1--3-2-2-1=-1-$ $9-1-6-4-6-\ldots--3-3-2-1--1-$ $10-1-6-5-6-2-2-2--3-3-2-1--1=$ $9-1-6-4-6--0-3-3-2-1--1-=$ $9=1-6-4-6=0=0-3-3-2-1=-1=0$ $9-1-6-4-6-0-3-3-2-1--1-=$ 7-1-6-4-6- - - - -3-2-2-1- -1- $8-1-7-5-6-2-2-4--4-3-3-1--1-=$ $16-1-7-4-7-3-3-1=-4-3-3-1=-1--$ $16-1-7-5-6-3-3-1--4-3-3-1=-1-$
$9-1-7-0-6-2-2-1--4-3-3-1--10$ $11-1-7-5-6-3-3-1=-4-3-3-1--10$ $11-1-7-4-6-3-3-1--4-3-3-1=-1-=$ $12-1-7-5-6-3-3-1=-4-3-3-1--1=$ $22-1-7-5-6-3-3-1--4-3-3-1--1=$ $4-1-7-5-6-3-3-1--4-3-3-1--1-$ $6-1=7-5-6-2-3-1=-4-3-3-1=-1<$ $12-1-7--3-3-1=-4-3-3-1--1=$ $12-1-7-=-3-1-1--4-3-3-1=-10$ $11-1-7-5-2-3-3-1--4-3-3-1--10-$ $16-1-7-5-6-3-3-1--4-3-3-1--1-$ $12-1-7-5-6-3-3-1--4-3-3-1--1=$ $8-1-7-5-6-3-3-1=-4-3-3-1=-1=-$
$8-1-7-5-6-3-3-1=-4-3-3-1=-1-$ $11-1-7-5-6-2-3-2--4-3-3-1--1-=$ $8-1-7-5-6-3-3-1--4-3-3-1--1-=$ $8-1-7-5-6-2-3-2--4-3-3-1=-1=$ $12-1-7-5-6-3-3-2--4-3-3-1--10-$ $9-1-7---3-3-1--4-3-3-1--1=-$
$6-1-7-5-6-2-2-2--4-3-3-1--1-$ $9-1-7-0-0-4-3-3-1--1-$ $9-1=7=6-8-2-2-2=-4-3-3-1=-10=$ $14-1-7-6-8-2-2-2--4-3-3-1=-1=-$ $16-1-7-5-6-2-2-3--4-3-3-1=-1=$ $6-1-7-4-6-3-3-1--4-3-3-1--1=$ 9-1-4-4-4-:-:-4-3-3-1--1-:$6-1-4-4-6--0-4-3-3-1--1=$ $6-1-4-4-6-3-3-1--4-3-3-1=-1-$ $9-1-7-4-6-\div=-4-3-3-1=-1=$ $11-1-7-5-6-3-3-1--4-3-3-1=-1=$
$9-1-7-5-6-2-3-2--4-3-3-1--1=$
SEDIMENT SAMPLES UNITS Ppm 3.10 3.10 3.30 
LASL Uranium Hydrogeochemical and Stream Sediment Reconnaissance Data Listing

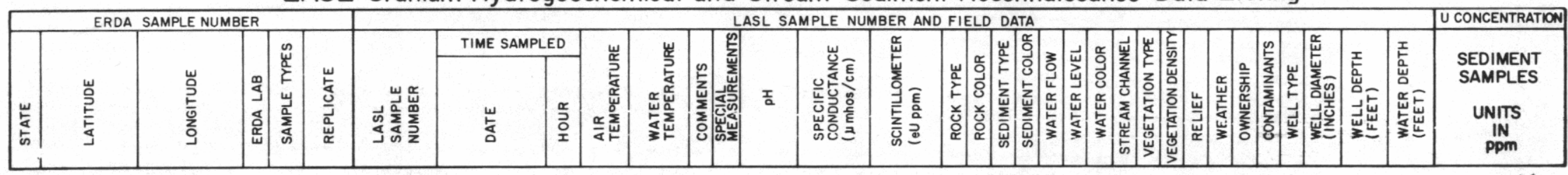

$36-43.7016-107.025 n-2-11-0-201495-n 7 / 88 / 74-10-30-12 . n-\ldots-7.2-500-50$ 56-43.1200-107.0411-2-11- $0-201496-07 / n 8 / 74-11-32-11 n . n-\ldots-7.2-450-$

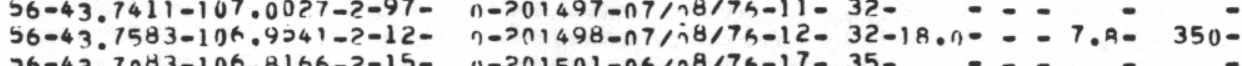

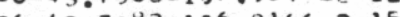
$36-43.7$. $36-43.7535-106.48 n 5-5-15-$ $36-43.6689-106.8156-2-14-$

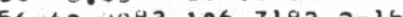
$56-43.9883-106.7183->-15-$ $56-43.9961-104.7172-7-12$ $56-43.9944-10607625-7-12-$ $56=43.9708-106.7847-2-14$ $36-43,9625-106.7041-7-12-$ $56-43.0944-106.7341-2-14=$ $56-43.9283-106.1133-7-14$ $36-43.893(1-106.336)=2-11$ $56-43.9125-104.8444-?-11-$ $56-43.9055-102.8055-2-11-$ $56-43.8261-104.6533-2-97$ $56-43.0255-10+.6501-?-15$ $56-43$. 8? $22-10 \% .7238->-15$ $36-43.8211-104,7288-?-97$ $56-43.8527-106,7177-?-14$ $36-43.5305-104.8625-2-12-$ $56-43.5319-10^{4} .8646-2-12-$ $56-43.525 n-104.9227-2-97$ $56-43.5111-106.725 n-2-15$ $56-43.5316-107,0141-2-15$ $56-43.5255-107.0311-?-11-$ $56-43.5633-107 \cdot 0125-2-97$ $56-43.5583-107 \cdot 0377-?-11=$ $26-43.543 n-107.0613-?-11-$ $56-43,5305-107.0727=?-11$ $56-43.5294-107.0761-2-12$ $36-43.5561-107.0668-2-11$ $56-43,5401)-107 \cdot 11(0-2-12-$ $36-43,5702-107,0834-2-11-$ $56-43.5716-107 \cdot 115 n-2-11-$ $36-43.5950-107.1044-2-11-$ $56-43.1633-167.1113-2-11-$ $56-43 \cdot 1844-107 \cdot 0527-2-11-$ $56-43.7811-107 \cdot 1183-2-11-$ $56-43.7938-107.1033-2-11-$ $36-43.8044-107 \cdot 1113-2-11-$ $56-43.8238-107.0868-2-11$ $56=43.0133-107.0488-7-12-$ $56-43.0583-10^{7} \cdot 0761-7-11-$ $36-43.8402-107 \cdot 0738-2-11-$ $56-43,083 a-107,08 ; ; 0-2-14$ $56-43.8977-107.0766-?-11=$ $n=>01501-n k / n 8 / 7 h-17-35$

$-201502-n 7 / 78 / 7 h-17-35-20=-0.0$
$-201504-07 / n 8 / 7 h-20-28-26.0=-0.9-6000-$

$n->01507-n 7 / i 9 / 76-17-36-15.0-\ldots 6.0-750=$ $n-201508-07 / 29 / 7 a-17-36-1400-=-6.8-700-$ $-201509-07 / 49 / 74-17-36-23.1--7.2014000$ -701510-07/i9/7h-18-36-19.7- - 7.4- 8ño $-201511-07 / 1017 n-18-36-190-18=-6.8-1800-$ - $01512-07 / 39 / 76-18-36-20.11=-0.6 .8-1200-$ $\rightarrow 01513-07 /$ i9/7a-20-27-16.0- - 8.5- $7 n 0$ 1-?01514-07/1,9/74-20-27-19.1- - 8.?- 5 nno-

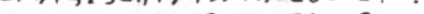
$-201517-07 \operatorname{lin} 9 / 74-21-25$

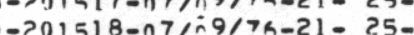

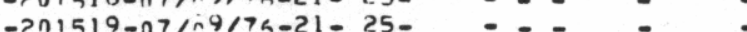

$-201520-07 / 109 / 75-21-25-21 . n-:-8.2-1900=$ :-201522-07/10/74- 7- 18-15.n- - O.A- $1100-$ -201523-07/10/7a- 7- 18-15.n- - - 6.8- $1100-$ $-201524-07 / 10176-7-19-$

- >01525-07110/7h- 7- 18

-7C1528-07/10/74-9-27- - - : -

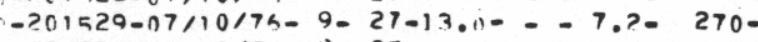
$\rightarrow->0153 n-1711017 a-9-27-$

$->01531-07 / 10 / 7 h-9-27-12 . n--0.9-250-$ $\rightarrow 01532-07 / 10 / 76-10-28-8 . n-$ - - 6.5- $260-$ (1-201533-07/10/74-10- 28- h.n- - - 6.7- 240 $n->01534-07 / 10 / 7 h-10-28-15.0-$ - $6 . h-300-$ $n-201535-07 / 10 / 7 n-11-31-13.0-=-6.8-350-$ n-?0153h-9?/i0/7a-11-31-14.0- - 7.4- 310$-201537-07 / 10 / 7 n-11-31-11.0=-6.3-300-$ n->01538-07/10/7h-11- 31-9.c- - - 6.8- $165-$ $n-201539-07 / i 0 / 76-12-32-i 4.11=--7.7-260-$ $-201540-07 / 10176-13-35-17 . n--6.9-150-$ ->01541-07/10/7h-13-35-15.n- - - 6.9- 2no$->01542-07 / 10 / 76-14-35-19.0--6.5-210-$ $\rightarrow 01543-07 / 1017 h-14-35-12.10-5.8-50-$ - $201544-07 / 10 / 7 h-14-35-10 . n--5.7-50=$ - $61545-07 / 16 / 76-14-36-12.0=-5.2-40-$

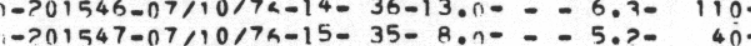
$0 \rightarrow 01548-07 / 10 / 75-15-35-9.0-=-5.5-50-$ $11->01549-07 / 10 / 7 h-15-35-13.0=-6.4-350-$ $\rightarrow 01550-0711017 k-15-35-21 . n-:-6.3-250$ 1->01551-07/10/7h-15-35-15.0- - - 6.2. 300-
- $201505-07 / 108 / 75-18-28-18.11-=-6 . h=7000-$ $-201506-07 / 19 / 7 a^{2}-17=36-$
$8-1-7-5-6-3-3-1--4-3-3-1--1-$ $9-1-7-5-6-3-3-1=-4-3-3-1--1=-$ $8-1-4-4-4--1-1-4-3-3-1--1=$
$9-1-4--1=-3-3-1=-4-3-3-1=-1=$ $12-1-4-4-4--1--4-3-2-1=-1=$ $12-1-4-4-4----4-3-2-1--10-$ $11-1-4-5-7-3-3-1--4-3-2-1--10-$ $9-1-7-4-7-3-3-1--4-3-2-1=-1=$ $9-1-7-5-6-3-3-1--4-3-2-1=-1-$
$1-1-$ $11-1-7-5-6-3-3-1=-4-3-2-1--1-$
$11-1-7-5-6-3-3-1--4-3-2-1=-1-$ $9-1-7-4-6-3-3-1--4-3-2-1--1-$ $9-1-7-5-6-3-3-1=-4-3-2-1=-1-$ $11-1-7-5-6-3-3-1=-4-3-2-1=-1=$ $8-1-7-5-6-3-3-1--4-3-3-1=-1-$
$11-1-7-5-6-3-3-1--4-3-3-1=-1-$ $1-1-7-5-6-3-3-1--4-3-3-1=-1=$
$9-1-7-5-6-3-3-1=-4-3-3-1--1=$ 6-1-7-5-6- - - -4-3-2-1- -1$11-1-7-5-6-\ldots-4-3-2-1--1-$ 8-1-7-5-6- - - -4-3-2-1- -1$11-1-7-5-6-1-1-4-4-2-1=-1=$
$11-1-7-6-8-3-3-1=-4-3-2-1=-1=$ $3-1-4-5-4-3-3-1--4-3-2-1--1-$ $3-1-4-5-4-3-3-1--4-3-2-1--1-=$ $6-1-4-5-6-1=-2-4-3-2-1=-1-$
$12-1-7-4-6-=-3-3-2-1=-1=$ $14-1-4-5-6-\cdots--4-3-2-1--1-1$ $16-1-4-5-6-3-3-1--4-3-2-1--1-$ $12-1-4-5-6-1-1-4-3-2-1--1=$
$14-1-4-5-6-3-3-1--4-3-2-1=-1=$ $11-1-4-5-6-3-3-1--4-3-2-1--1-$ $6-1-4-5-6-3-3-1--4-3-2-1--1-$ $6-1-4-5-6-3-3-1-4-4-3-2-1--1=$
$12-1-4-5-6-3-3-1-=4-3-3-1--1=$ $12-1-4-5-6-3-3-1-4-4-3-3-1=-1=$ $12-1-4-5-6-3-3-1<-4-3-2-1<-1-$ $9-1-4-5-6-3-3-1--4-3-4-1--1=-$ $9-1-7-5-6-3-3-1--4-3-4-1--1<$ $16-1-7-5-6-3-3-1--4-3-4-1--1-$ $16-1-7-6-8-3-3-1--4-3-4-1--1-$ $9-1-7---3-3-1--4-3-4-1--1-=$ $12-1-7-5-6-3-3-1--4-3-4-1--1=-$ $6-1-7-5-4-3-3-1--4-3-4-1--1=$ $3-1-7--3-3-1--4-3-4-1--1-$
$9-1-7-5-6-3-3-1--4-3-4-1--1-$ $8-1-7-5-6-3-3-1--4-3-4-1--1--$
$2-1-7-4-6-3-3-1--4-3-3-1--1=-$ $12-1-7-4-6-3-3-1--4-3-3-1--1=$ $16-1-7-5-6-3-3-1--4-3-4-1--1=-$
$3-1-7-5-6-3-3-1--4-3-3-1--1=$

3.20
2.80
2.10
2.20
2.30
2.100
5.20
4.50
4040
3.10
3.10
4.10
2.60
4.10
4030
2.30
2.10
2.10
2.70
2.70
2.60
2.70
2.70
2.70
2.90
3.90
2.80
3.40
2.00
3.40
2.50
4.00
2.50
2.80
2.60
3.30
2.40
5.30
5.00
3.80
3.70
2.90
3.40
3.90
6.50
3.10
3.00
2.70
2.80
3.50
2.60


LASL Uranium Hydrogeochemical and Stream Sediment Reconnaissance Data Listing

\begin{tabular}{|c|c|c|c|c|c|c|c|c|c|c|c|c|c|c|c|c|c|c|c|c|c|c|c|c|c|c|}
\hline \multirow[b]{3}{*}{ 嵌 } & \multicolumn{5}{|c|}{ ERDA SAMPLE NUMBER } & \multirow[b]{3}{*}{ 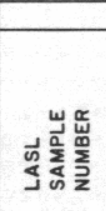 } & \multirow{2}{*}{\multicolumn{2}{|c|}{ TIME SAMPLED }} & \multirow{3}{*}{ 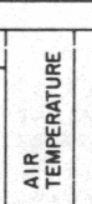 } & \multirow{3}{*}{ 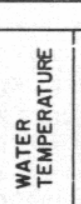 } & \multirow{3}{*}{ 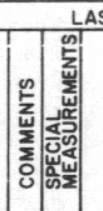 } & \multirow[b]{3}{*}{ I⿳亠口冋口厶 } & \multirow[b]{3}{*}{ 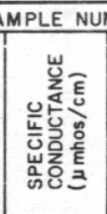 } & \multirow{3}{*}{ 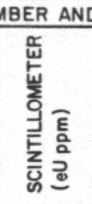 } & \multirow{3}{*}{ 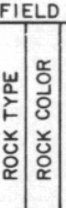 } & \multirow{3}{*}{ 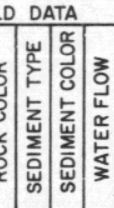 } & & & & & & & & & & $U$ CONCENTRATION \\
\hline & & & & $\psi$ & & & & & & & & & & & & & \multirow{2}{*}{\multicolumn{3}{|c|}{ 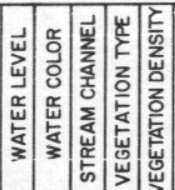 }} & & \multirow{2}{*}{\multicolumn{3}{|c|}{ 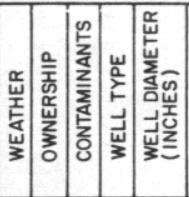 }} & \multirow[b]{2}{*}{ 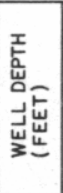 } & \multirow{2}{*}{ 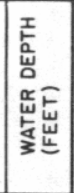 } & \multirow{2}{*}{$\begin{array}{c}\text { SEDIMENT } \\
\text { SAMPLES } \\
\text { UNITS } \\
\text { IN } \\
\text { Ppm }\end{array}$} \\
\hline & 㞺 & 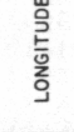 & 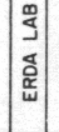 & 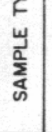 & $\begin{array}{l}\frac{5}{b} \\
\frac{d}{a} \\
\frac{a}{a} \\
\alpha\end{array}$ & & 离 & 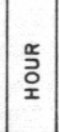 & & & & & & & & & & & & & & & & & & \\
\hline
\end{tabular}

b6-43. YnO3-107.0283-7-12- 1-?61552-07/10/74-16-36-12.0- - 6.5- $270-$ $36-43.8138-106.3361-2-11-0-701554-07 / 10 / 74-17-37-18 . n-0-7.2-2400-$ 56 040 . $56-44.2416-104.9633-2-12=$ $36-44.2427-106.9638-7-15$ $56-44.1294-106.9101-7-12$ $56-44.1855-107.0119-5-12=$ $56-44.1711-107 \cdot 0119-2-11$ $56-44.1452-107.0644-2-11$ $56-44.1452-107.0644-5-11=$ $56-44.1322-107.1205-3-12-$ $0.44 .1061-107.1100-5-15-$ $36-44.0977-107.0938-7-12-$ $10-40131107.1052-2-11=$ $36-44$.11311-107.105?-?-11$56-44.0172-107.0938-7-11-$ $56-44.0255-107.0227-?-12=$ $36-44.0133-104.9936-?-12-$ $56-44.01111-10 \mathrm{0h} .9105-2-11-$ $56-44.0144-106.9483-2-11=$ $56-4,0177-104.9244-?-11$ $56-44.13272-104.0955-2-11-$ $56-44.0238-104.8683-?-11-$ $10-44027701008025-110$ $56-44.0438-10 \% .8305-2-11$ $56-44.0305-106.9261-2-12$ $36-44.0313-10 k .9255-2-12-$ $56-44.0644-106.8761-2-15$ $56-44.0694-102.9005-2-12-$ $36=44.0827-10 \% .8819-2-11-$ $56-44$. $0827-10 \mathrm{~K}, 9055-2-11$ $36-44.0933-10 \% .8727-2-15$ $56-44.0927-10 k .871$ has- $-11-$ $56-44$. 1 n $94-10+.8511-2-11$ $56-44.0171-107.0527-2-12-$ $56-44.0038-107.0705-2-14=$ $36-43.9872-107.0366-2-14$ $56-43.7788-107.0594-2-14$ $56-43,9805-107,1194-2-11$ $56-43 . y 783-107 \cdot 0338-2-14-$ $56-43, y 616-107.0744-?-14$ $56-43.9511-107.057 ?-2-14-$ 26-43.9555-107.0327-?-11$56-43.7577-107.0472-2-11-$ $56-43.9283-107.0911-7-11$ $56-43.7 ? 30-107.078,->-11-$ $56-47,7127-107,0516->-11$ $56-43.9044-107.0338-2-11-$ $56-43.8972 .-1 U^{4} .9875-2-11$ $36-43.8597-106.9666-2-15-$ $56-43.0694-176.9138-?-12$ $n \rightarrow 01558-n 7 / 13 / 7 k-10-24-15$

- $01559-n 7713775-10-24-$ - -

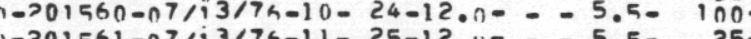
$n \rightarrow 01562-07 / 13 / 7 a-11-25-15 . n-=-5.5055$ 源 $-701564-n 7 / 13 / 7 h-12-26-13 . n-0-5.2-50$ $->01565-n 7113 / 7 a-12-26-17.00--5.7-60-$ . - $201567-n 7113,75-13-26-17 \cdot 0=-5.7-$ $-701569-n 7 / 13 / 79-14-29-20.00=-5.9-$ $-201570-07 / 13 / 7 a-14-29-11.0-=50.0-$ $-201571-07 / 13 / 74-15-29-18,0-0-5,7-$ $\rightarrow 0157>-07 / 13 / 74-15-29-19.00-0.5 .90$ -201573-n7/13/7a-16-23-13.n- - $5.7-$ n-201574-07)i3/75-16-23-20.0- - - 6.3$->01575-071317 k-17=23-13 \cdot 0=-5.9-$ $1=201577-07 / 3175-17-23-12.00=-5.7-$ 作 $n=201579-n 7 / 13 / 7 k-17-23-12 . n-$ - - 5.9$n \rightarrow 001580-07 / 13 / 76-18-23-17 \cdot 0-=-5.7-$
$n \rightarrow 001581-n 7 / 13 / 7 a-18-23-15.0-0-50$ $-201582-n>1,13 / 7 n-18-23-$

作 (160) $n-201585-07 / 13 / 79-19-22-13.11=-5.7-$ $n=201586-0713 / 75-19-22-$

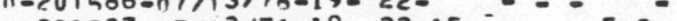
$0=201588-07 / 13 / 76-19-22-13 . n-=-5.9-80-80$ $n-? 01589-n 7 / 14 / 7 a-8-15-1 \hat{n} \cdot n-=-5.5-30-$ $3-201590-07 / 14 / 76-8-15-12.0=-6.3-150$ ค-201591-07/14/74- 8- $15-9.0=-0.3=270$ $n=201592-07 / 14 / 7 h-9-20-14 \cdot 1)=-5 . ?-? ?-$

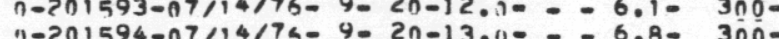
$n-201594-07 / 14 / 74-9-2 n-13.0)-0.8-3 n 0-$
$n-201595-07 / 14 / 74-9-20-11 . n-0-6.5-35 n-3$ $n-201595-07 / 14 / 7 h-9-20-11 . n-0-6.5-35 n-$
$n-201596-07 / 14 / 7 h-9-20-12 . n-0-6.1-340-$ $0=201597-n 7 / 14 / 74-10-20-10.0=-6.5-400-$ $n=201598-07 / 14 / 9 h-10-20-9.0-=-6.10290$ $n-201599-07 / 14 / 74-10-20-10.0-=6.5-300-$ $n-201600-07 / 14 / 74-10-20-9 . n=-6.3-270-$ $n-701601-07 / 14 / 74-11=25-9 . n-=-6.8=350$ $n=201602-n 7 / 14 / 74-11-25-11 \cdot 01-=-6.5-375-$ $n-? 01603-n 7 / 14 / 7 h-11-25-1 \mathrm{~h} \cdot n=-6.8-330^{\circ}$ $n-201604-n 7 / 14 / 74-11-25-$
$n-201605-n 7 / 14 / 74-12-25-15 . n-:-6.4-325-$
$12-1-7-5-6-3-3-1=-4-3-4-1--1-$ $8-1-7-5-6-3-3-1--4-3-3-1--1-$ 1 $2-1-7-5-8-3-3-1=-4-3-4-2=-1=$ $12-1-7-5-8-3-3-1-2-4-3-4-2-1-$ $6-1-7-5-6-3-3-1=-4-3-4-2--1=$ $3-1-7-4-6-3-3-1--4-3-2-2--1-=$ $4-1-7-4-6-3-3-1--4-3-4-2--1-=$ $4-1-7-4-6-3-3-1=-4-3-4-2--1=$
$6-1-7-5-6-3-3-1=-4-3-4-2--1=$ $4-1-7-4-6-3-3-1--4-3-4-2--1=$ $6-1-7-4-6-3-3-1--4-3-4-2--1-$ -6-3-3-1- $-4-3-4-1=-1-$ $9-1-7-4-8-3-3-1=-4-3-4-1--1=$ $9-1-7---3-3-1--4-3-4-1--10=$ $9-1-7-7-6-3-3-1=-4-3-4-1--10$
$1-1-7-5-6-3-3-1--4-3-4-1--1=$ $12-1-7-5-6-3-3-1--4-3-4-1=-10$ $8-1-7-5-6-3-3-1--4-3-4-1--1-$ $6-1-7-0-3-3-1=-4-3-4-2=-1=$
$9-1-7-5-6-3-3-1-0-4-3-4-1--1=$ $8-1-7-5-6-3-3-1=-4-3-4-1=-1=$ $8-1-7-5-6-3-3-1=-4-3-4-1-1-1=$
$11-1-7-5-6-3-3-1-04-3-4-2--1=$ $8-1-7-5-6-3-3-1--4-3-4-2--1-=$ $8-1-7-5-6-3-3-1-4-4-3-4-2--1=$
$8-1-7-5-6-3-3-1--4-3-4-1--1=$ $11-1-7-5-6-3-3-1--4-3-4-1--1-=$ $11-1-7-5-6-3-3-1--4-3-4-1--1-$
$9-1-7-4-8-3-3-1--4-3-4-2--1-$ 3-1-7-4-8-3-3-1- -4-3-4-2--1- $9-1-7-4-6-0--4-3-4-1--1-$ $6-1-7-4-6-3-3-1=-4-3-3-1=-1=$ $9-1-7-4-6-3-3-1--4-3-4-1=-1=$
$9-1-7-4-6-3-3-1--4-3-4-1--1=$ $9-1-7-4-6-\cdots--4-3-4-1--1-$ $3-1-7-4-6-3-3-1=-4-3-4-1=-1-$ $9-1-7-5-6-3-3-1--4-3-4-1--10$ $11-1-7-4-6-3-3-1=-4-3-4-1--1=$ $12-1-7-4-6-3-3-1--4-3-4-1--1=$
$8-1-7-5-6-3-3-1=-4-3-2-1--1=$ $8-1-7-5-6-3-3-1--4-3-4-1--10=$ $9-1-7-5-6-3-3-1--4-3-4-1=-1-$ h-1-7-5-6-3-3-1- $-4-3-4-1--1=-$
$11-1-7-5-6-3-3-1--4-3-3-1--1=-$ $12-1-7-4-4-3-3-1=-4-3-4-1=-1=-$ $11-1-7-5-6-3-3-1=-4-3-4-1=-1-=$
$9-1-7-5-6-3-3-1--4-3-3-1=-1-=$ $4-1-7-5-6-3-3-1--4-3-4-1--1-$ $9-1-7-5-6-3-3-1--4-3-3-1--1=-$ $6-1-7-5-6-3-3-1=-4-9-3-1=-1-:$
$8-1-7--3-3-1=-4-3-4-1=-1=$ $8-1-7--3-3-1=-4-3-4-1--1=$
$6-1-7-5-6---1--4-3-4-1--1-$ $6-1-7-5-6-1-2-4-3-4-1--1-$
$9-1-7-5-6-3-3-1--4-3-4-1--1=$
1.80

1.80
1.80

4.70

1.80

3.90

1.40

3.20
3.50

2.10

3.10

3.90

2.30

3.40

3.50

$3.80^{\circ}$

3.50

4.60
3.70

3.70

3.70

3.70

4.20

2.00

1.40

3.20

3.30

3.70

3.10

3.40

4.50

1.80

2.40

4.20

2.00

2. 00

3.10

0.80

2.70

2.20

2.80

2.90

2.90

$2.50^{\circ}$
$2.40^{\circ}$ 
LASL Uranium Hydrogeochemical and Stream Sediment Reconnaissance Data Listing

\begin{tabular}{|c|c|c|c|c|c|c|c|c|c|c|c|c|c|c|c|c|c|c|c|c|c|c|c|c|c|c|c|c|c|c|}
\hline \multirow[b]{3}{*}{$\mid$} & \multirow{3}{*}{ 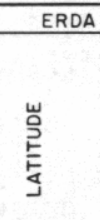 } & \multicolumn{4}{|c|}{ SAMPLE NUMBER } & \multicolumn{24}{|c|}{ LASL SAMPLE NUMBER AND FIELD DATA } & \multirow{2}{*}{ U CONCENTRATION } \\
\hline & & & & $\ddot{\psi}$ & & & TIME $\mathrm{S}$ & & & & & & & & & & wa & & & & & & & & & & 蔍 & & I & \\
\hline & & 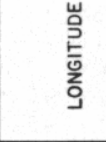 & 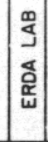 & 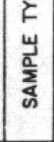 & 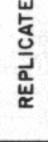 & 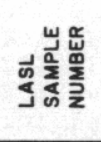 & 岁 & 崩 & 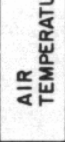 & 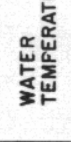 & 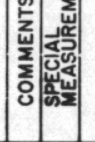 & $\mathbf{I}$ & 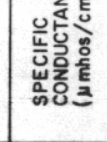 & 訔 & 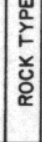 & 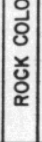 & 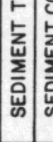 & 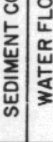 & 焉 & $\begin{array}{l}0 \\
0 \\
\frac{x}{w} \\
\frac{4}{3}\end{array}$ & 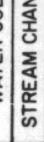 & 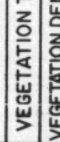 & 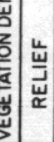 & 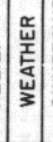 & 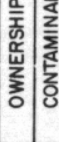 & $\sum_{0}^{2}$ & 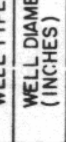 & 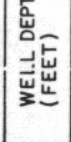 & 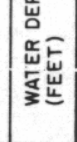 & $\begin{array}{l}\text { SAMPLES } \\
\text { UNITS } \\
\text { IN } \\
\text { Ppm }\end{array}$ \\
\hline
\end{tabular}

$56-43.0694-104.9055-2-11-$ $36-43.8472-106.9694-2-11-$ $56-43.0333-10+.9639-2-11-$ $56-47.4150-106.9611-2-11-$
$36-43.77+1-106.92511-2-15-$ $36-43.7731-106.92511-2-15-$
$36-43.7905-106.9069-2-11-$ $36-43,0206-107.0975-2-11-$ $36-43,0206-107.0975-2-11-$
$56-44,+15,-105.0966-2-15-$ $56-44, y \rightarrow \rightarrow 7-10=.0161-2-97-$ $36-44$. $7477-10=0161-2-97=$ $56-44, y 561-105.037>-2-15-$ $36-44,743:-105.0288-2-15-$ $56-44,7431-105.0288-2-15-$
$56-44,7425-105.0311-2-15-$ $56-44 .+361-105.0611-2-15-$ $56-44.9321-105.150 n-2-15-$ $56-44.2733-105.2450-2-15-$ $36-44,2572-105.2116-2-14=$
$36-44,210,1-105.2139-2-97=$ $56-44,2627-105.1716-2-97=$ $56-44.2338-105.1883-2-15-$ $56-44.2316-105.1569-2-14-$ $56-44.2316-105.1569-2-14-$
$56-44.1627-105.1727-2-15-$ $56-44.1627-105.1727-2-15-$
$36-44,8683-105.0727-2-14-$ $36-44.8683-105.0727-2-14-$
$36-44.053 H-105.0091-2-14-$ $36-44.055 H-105.0391-2-14-$
$56-44.0702-105.035 ?-2-14=$ $56-44.0702-105.035 ?-2-14=$
$56-44.8563-105.0066-2-14=$ $56-44.8563-105.0066-2-14=$
$36-44.8488-105.0725-2-14-$ $56-44.0233-105.0713-2-14-$ $56-44.0180-105.0255-2-14-$ $56-44,1605-105.007 ?-2-15-$ $56-44.7625-105 \cdot 0458-2-14=$ $36-44,7825-104.0616-2-14-$ $56-44.7841-105 \cdot 0863-2-14-$ כ6-44. 81111-105.0863-?-14$56-44$. ठग13-105.1041-2-15$56-44.0627-105.1491-2-15-$ $56-44$. $1905-105 \cdot 2236-2-15-$ $56-44.7605-105.178 n-2-15-$ $56-44.0813-105.2488-2-15-$ $56-44.565310-105.2319-2-15-$ $56-44.7413-105.22 n 2-2-15-$ $36-44.7400-105.1697-2-15-$ $56-44.0447-105.1258-2-15-$ $56-44.0566-10 \leqslant .1858-2-99-$ $56-44.5804-105.1536-2-15-$ $36-44.6783-105.10 \cap 4-2-15-$ $56-44.0977-105.083 h-2-15-$ $56-44,1083-105,0888-2-99-$ $56-44.1319-105.1155-2-97-$ $56-44.0686-10^{2} .0184-2-15-$ $56-44$. $1325-105.0247-2-15-$
$-201506-07 / 14 / 7 a-12-25-12 \cdot n--6.3-$ - $201607-07 / 14 / 7 k-13-26-12 \cdot n=-6.3-$ 作 (1) - $01610-07 / 14 / 7 a-14-28-$ - $201612-07 / 14 / 75-20-20-12.0-=6.0-250-$ - $01513-071250-10-27-$ $001615-07120174-10=27$ $-201616-07,32 / 79-11-37=$

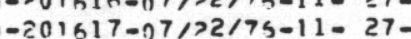
0
$->1618-07 / 72174-11-27-$ $-201623-071>2175-12-30-$ $-501535-177 / 25 / 7 h=13-33-$ $n \rightarrow>01643-n>1>4 / 76-10-30-25 \cdot n-C_{-}-7.5-23000-$ $-201649-07174174-12-36-0$ $n \rightarrow>01649-n>>>4 / 74-13-35-22 . n--6.3-1250^{\circ}$ $-201650-07 / 24 / 75-13-37-$
$-201651-07 / 72 / 7 n-10-27-22 . n=-0.6-19 n 0-$ $-20165 ?-07 / 22174-10-27-21 . n--7 . n-200-$ ->01653-n7/32/7h-1n- 27-25.5- - - 7.?- 6no$n-201654-07 / 22 / 7 h-11-27-22 \cdot 0-=-7 \cdot 1-450-$
$-201655-07 / 22 / 7 a-11-28-20 \cdot 0-0-7.1-700-$

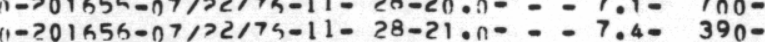
$n \rightarrow 001557-n 7 / 2217 n-12-28-22 . n-0-7.20550-$ $n-201558-n 7 />2 / 7 h-12-28-$
$n-201659-07 />21>4-12-30-19 . n-0-7 . n-7 n 0-$

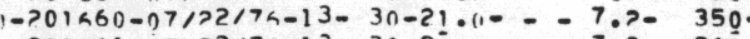
$n=>1661-n>13217 n-13-3 n-2 n \cdot n-0-7.8-260-$ $1-20166>-07 / 22 / 7 h-13-30-22.5=-0.8-6 n 0-$ $n \rightarrow>01663-n 7 / 32 / 7 n-13-30-$ $11-201664-n 7 / 22 / 7 h-14-30-$ $n \rightarrow>01669-n 7 />2 / 75-16-32-$ 0
$n-20167167-n=07 / 253 / 74-11-31-$ $n-201677-0,07 / 23 / 7 n-11-31=$
$n \rightarrow 201676-n 7 / 23 / 7 k-11-31-$ $n-201677-07 / 23 / 7 h-11-31-$ $n-201679-07 / 23 / 76-11-32-$ $n \rightarrow>0168$ ? $-07 / 73 / 74-12-32-$ $n=201684-07 / 23 / 75-13-33-$ $n=201685-n 7 / 23 / 75-13-33-$ $\rightarrow 01687-n 7 / 23 / 7 h-14=33$ $n-201688-n 7 />3 / 7 h-14=33-$ - $-201590-n 7 />3 / 76-15-32$ $n->01692-07 / 33 / 7 n-16-32-$ - $-201593-07 / 33 / 76-16-32$ $1=201694-n 7 / 23 / 75-17=3 n-$
$0->01695-n 7 / 23 / 74-17-3 n=$
$11-1-7-5-6-3-3-1=-4-3-4-1--1-=$
$6-1-7-5-6-3-3-1--4-3-4-1--1-$

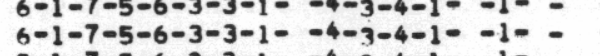
$6-1-7-5-6-3-3-1-4-3-4-1--1=$
$9-1-7-5-6-3-3-1--4-3-4-1--1=$ $9-1-7-5-6--0-4-3-4-1--1-$ $11-1-7-5-6-3-3-1--4-3-2-1--10$ $9-1-7-5-6-3-3-1--4-3-4-1--1-=$
$7-1-6-4-6-0-1--4-2-2-1--1=$ $7-1-6-4-6-1-6-4-6--1-0-4-2-1-1--1=-$
$7-1-6-4-6-1-$ $7-1-6-4-6--1=-4-2-1-1--1=$
$7-1-6-4-6--1--4-2-1-1--1=$ $7-1-6-4-6--1---4-2-1-1--1-$
$6-1-6-4-6--1--4-3-1-1--1-$ $6-1-6-4-6--1=-4-3-1-1--1-$
$9-1-6-4-6--1=-4-3-1-1--1-$ $12-1-6-4-6--1--4-3-1-1--1-$ $4-1-6-4-6--1=-4-2-2-1=-1-$
$6-1-7-4-6--1-2-4-3-4-1=-1-$ $13-1-6-4-6-1=-4-2-2-1=-2-$ $12-1-6-5-8-=-2=-4-2-2-1=-2-=$ $7-1-6-4-6-\cdots--4-2-2-1--1-$

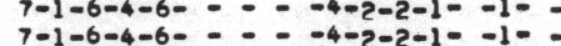
$10-1-6-5-6-2-2-2--4-2-2-1--1=$ $9-1-6-4-6-1-3-5-4-4-2-2-1--1-$
$8-1-7-4-3-5-2-1--1-$ $9-1-7-5-6-1-3-5--4-3-2-1=-1-$ $10-1-7-5-6-1-3-5--4-3-2-1--1=$ $10-1-7-5-6-1-3-5--4-3-2-1--1-$ $12-1-7-5-6-1-3-5--3-3-2-1=-1-$ - $1-7-5-6-1-3-5--3-2-2-1-=10$ $11-1-7-4-6-1-3-5--3-3-2-1=-1=$ $4-1-7-4-6-1-3-3--4-3-2-1--1-$ $7-1-7-6-7-1-3-3--4-3-2-1--1=$ $12-1-7-5-6-1-3-3--4-3-2-1=-1=$ $12-1-7-4-6---4-3-2-1--1-$ 8-1-7-5-6- - - - -4-3-2-1--1$11-1-7-5-6-0=-4-3-2-1--1-$
$9-1-7-5-6-0$ $11-1-7-4-6-0--2-5-2-1=-10=$ $8-1-7-4-6--0-3-3-2-1--1-=$

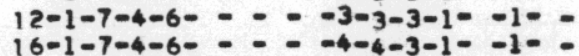

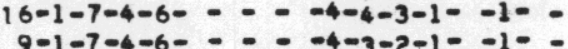

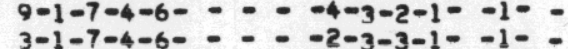
$14-1-7-5-6-\cdots--4-3-2-1--1-$ $11-1-7-5-6-\cdots--2-3-2-1--10=$ $6-1-7-5-6-\div-04-4-2-1=-1=$
$6-1-7-4-6-\div-04-4-3-1=-1-$ $6-1-7-4-6-\div-2-4-4-3-1=-1-$

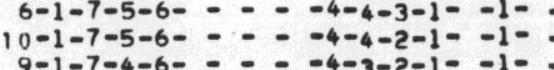

2.00

2.50

2.30

2.50

2.40

2.40

3.10

3.00

2.70

2.80

2.60

2.90

3.20

3.20

2.90

2.70

3.40

3.30

3.80

2.60

2.60

3.40

3.20

3.00
2.10

2.90

2.60

3.10

2.90

1.80

3.80

4.70
2.90

2.50

2.50

2.90

2.80

2.60

3.50
2.70

$2.60^{\circ}$

2.90

3.40

3.00

3.40
2.90 
LASL Uranium Hydrogeochemical and Stream Sediment Reconnaissance Data Listing

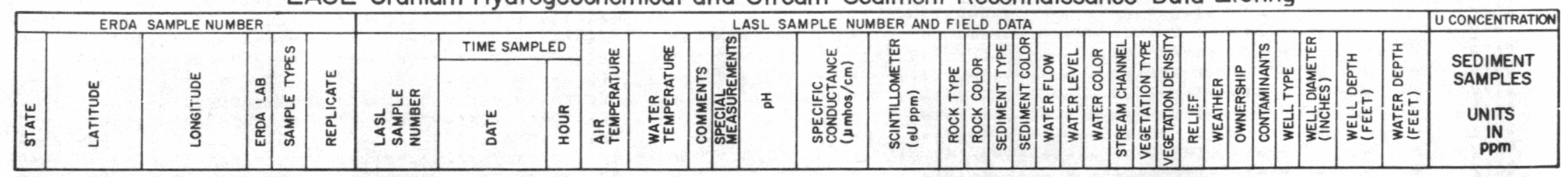

$56-44.7352-105.9422-?-15-n-701698-n 7 / 33 / 79-18-3 n-$ $36-44.3938-105.8027-2-15-n->01701-1 n / n 8 / 7 h-13-21-$ $56-44.3791-105.8069-2-15-$ $56-44.3775-105 \cdot 7902-2-15-$ $36-44 \cdot 1444-105 \cdot 9055-2-15$ $56-44.151 y-105.905 n-2-15-$ $56-44.1733-105.8658-?-15-$ $56-44.1577-105.7858-?-15-$
$56-44.1530-105.7736-2-97-$ $56-44.1530-105.7736-2-97-$
$56-44.1863-105.8458-2-15-$ $56-44.1863-105.8458-2-15-$
$56-44.2263-105.7991-?-15-$ $56-44.2263-105.7991-2-15-$
$56-44.1138-105.8666-2-111-$ $56-44.1138-105.8666-2-11-$
$56-44.0950-105.8227-2-15-$ $56-44.0791-105.7711-2-15-$ $56-44.0694-105.7577-2-11=$ $36-44.0327-105.7811-2-11-$
$56-44.0011-105.7804-2-97-$ $36-44.0069-105.8305-2-15=$ $56-44.0241-105.8419-2-15-$ $36-44.0244-105.8255-2-15-$
$56-44.0783-105.9647-2-15-$ $56-44.0847-105.9866-2-15=$ $56-44.0402-105.9755-2-15-$ $56-44.0327-104.0305-2-15-$ $56-44.0166-106.1153-7-15-$
$56-44.108 i)-104.1055-2-15-$ $56-44.1080-10 f .11055-2-15-$
$56-44.1000-104.0622-2-14-$ $56-44.1000-106.0622-2-14-$
$56-44.1011-106.0538-2-15-$ 56-44.0975-106.0516-2-15$56-44.1747-106.0405-2-15-$ $56-44 \cdot 1822-106.1011-2-15-$
$56-44.1725-105.9316-2-15-$ $56-44,1883-105.9366-2-15$ $56-44,1938-105.9727-2-15-$ $36-44.2105-105.4983-2-15-$ $36-44.2111-106.0133-2-15$ $56-44.220 n-10 \% .0227-2-15-$ $56-44.2166-106.057 ?-2-15-$ 56-44.2902-10f.115?-2-15$56-44.1994-104.9625-7-15-$ $56-44.1772-10+.100 n-2-15-$ $56-44.1305-106.1333-2-15-$ $56-44.095 n-106.1763-2-15-$ $56-44.0488-107.0055-2-15-$ $56-44.6508-107.015,-2-11-$ $56-44.6383-107.0236-2-15-$ $56-44.6297-107.0280-2-12-$ $56-44.6300-107.0244-2-15$ $56-44.6700-107.0052-2-15-$ $56-44.6672-107.0713-2-12-$
$56-44.6897-107.0838-2-12-$ -201704-10/n8/76-13- 21 $\rightarrow 01706-10 / 78 / 75-13-21-$ 1->01708-10/iib/75-14- 22 n-P0171?-1 $1 / 198 / 79-15-22$ $n \rightarrow 01716-10 / 78 / 75-15-22-$
$n->01717-1 n / n 8 / 7 a-16-21-$ $-201721-1 n / 18 / 75-17=20-$ -201723-1n/ri8/75-18- 18 $n=201728-1 n / \operatorname{sig/7a-8}-12$ $n-201733-1 n / n 9 / 7 a-90-14-$ $1)-201735-1 n / 19 / 7 h-10-16-$
$-201736-1 / n / n 9 / 7 a-1 n-1 b-$ $n-201736-1 n / n 9 / 7 a-1 n-1 b-$ $0=? 01737-16 / 99 / 74-10=16-$ $1=201746-10 / 19176-12-19$ $n \rightarrow 01750-1 n / n 9 / 7 a-12-19$ $n=201757-1 n / n 9 / 7 n-14-21-$ $n \rightarrow ? 01758-1 n / n 9 / 74-14-21-$
$n->1) 1763-1 n / n 9 / 7 a-15-2 n-$ $n-201770-1 n / n 9 / 75-18-14-$ ח- $01771-10 / 10 / 74-8-12$ $n=>01779-10 / 10 / 74-9=14-$

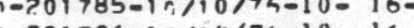
- $01786-1$ n $1117 / 7 n-10=16-$ n-201788-1 ? n- $0179 n-1 n / 10 / 75-11-18=$ 0
$0-201792-10 / 10 / 7 n-11$ $n-201795-101017 n-12-20$ $0 \rightarrow>01810-10 / 10 / 7 h-13-20$ $n=201 A 13-1 n / 10 / 7 a-14-2 n-$ $n=201816-1 n / 10 / 76-14-20=$ $n \rightarrow>01819-1 n / 10 / 7 b-15-2 n-$ (1)>0 $820-10 / 10 / 76-15-20-$ $n=201821-n 5 />66 / 76-12-16-$ $n=? 01702-1 n / i 8 / 76-13-21-$ $1-201726-10 / 119 / 75-8012-12.0=-0.9-5510=$ 1) $-201729-1 \mathrm{n} / 1917 \mathrm{~h}-9-14-$ - - - - $n=201730-1$ ing $9 / 74-9-14-12$-n- - 6.9-53ño$n=201732-1 n / n 9 / 7 n-9-14-11$.ne- - 6.9- $50000-$ -

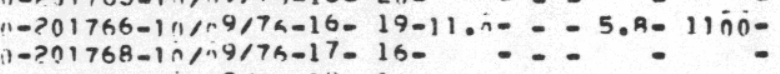

$\therefore$

$\because$

$-$

- -

$-$

- -

$\div:-$

$-$

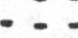

$-\cdot$

$\div-$

$2-$

$\div=$

- -

$\because-$

$4-1-7-4-6-\cdots--4-3-2-1--1--$ $1-1-7-5-6--1--2-3-3-2-1=-1=-$ $16-1-7-5-6--1--2-4-3-2-1--1=:$
$17-1-7-4-6--1--2-3-3-2-1--1=$ $12-1-7-4-6--1--2-3-3-2-1--1=$ $12-1-7-4-6--1=--3-3-2-1--1=-$ $11-1-7-4-6--1<-2-4-3-2-1--1-=$ $14-1-7-4-6--1--2-4-3-2-1--1--$ $16-1-7-4-6--1--2-4-3-2-1--1=$ $14-1-7-4-6--1--2-4-3-2-1--10=$ $6-1-7-4-6--1--2-4-3-2-1=-1-$ $8-1-7-5-8-3-3-1=-4-3-2-1=-10=$ $9-1-7-5-6--1=-2-4-3-2-1=-10=$ $1-1-7-5-6--1--2-4-3-2-1--1=-$ $9-1-7-5-6-3-3-1-3-4-3-2-1=-1-=$ $14-1-7-5-6--1--2-4-3-2-1--1=-$ $14-1-7-5-6--1--2-4-3-2-1--1<=-$ $11-1-7-5-6--1--2-4-3-2-1=-10=$ $\left\{\begin{array}{l}1-1-7-4-6--1--2-4-3-2-1--1:= \\ 17-1-7-4-6--1--2-3-3-2-1--10=\end{array}\right.$ $21-1-7-4-6--1--2-3-3-2-1=-1-=$ $8-1-7-4-6--1--2-3-3-2-1--1-=$ $12-1-7-5-6-1--2-4-3-2-1--1-$ $11-1-7-5-6--1=-2-4-3-2-1=-1=-$ $12-1-7-5-6-3-3-1-3-3-3-2-1--10=$ $25-1-7-5-6---2-4-3-2-1--1--$ $19-1-7-5-6--1--2-4-3-2-1--1=-$ $8-1-7-5-6--1--2-4-3-2-1--1-$ $16-1-7-4-6--1=-2-4-3-2-1=-1=-$
$16-1-7-4-6--1--2-4-3-2-1=-1-=$ $16-1-7-4-6--1--2-4-3-2-1=-1=-$
$16-1-7-4-6--1--2-4-3-2-1--1=$ $16-1-7-4-6--1--2-4-3-2-1--1-=$
$19-1-7-4-6--1--2-4-3-2-1=-10=$ $19-1-7-4-6--1--2-4-3-2-1=-1-=$
$12-1-7-4-6--1--2-4-3-2-1=-1-=$ $14-1-7-4-6--1--2-3-3-2-1=-1-$ $11-1-7-4-6--1--2-4-3-2-1=-10$
$11-1-7-4-6--1--2-4-3-2-1=-1=$ $11-1-7-4-6--1--2-4-3-2-1=-1=$
$14-1-7-4-6--1=-2-4-3-2-1=-1=$ $12-1-7-4-6--1=-2-4-3-2-1=-1-$ $16-1-7-4-6--1---4-3-2-1=-10$
$16-1-7-4-6--1--2-4-3-2-1=-1:$ $16-1-7-4-6--1--2-4-3-2-1=-1-$ $12-1-7-7-8-1-1--2-3-2-1=-1=$
$3-1-6-6-6-2-3-1--2-3-2-1=-1=$ $14-1-6-4-6-1-1--2-4-2-1=-1=$
$5-1-8-4-8-4-4-1-2-2-3-2-2-1=$ $5-1-8-4-8-4-4-1-2-2-3-2-2--1=$
$8-1-6--8-1-1--2-3-2-2--10$ $6-1-6-5-6-6-2-3-3-2--1=$
$5-1-6-5-6-3-4-1-2-2-3-2-1--10$

$5-1-6-5-6-3-4-1-2-2-3-2-1--1-$
$10-1-6-4-6-2-4-1-1-2-3-2-1--1-$

2.80

.5

3.70

4.40

3.60

3.00

3.80

4.000

4.00

2.80

3.30

30

400

5.10

5.40

490

.30

3.70

3.50

4.20

(1)

3.60

4.50

.

3.60

430

4.30

3.60

4.80

.70

3.60

2.80

3.40

3.10

3.90

3.60

4.00

3.60

2.20

4.30

2.50

2.10

.90

20

3.30

2.00

2.50

2.50

$n-201833-02 / 26 / 76-13-20-\quad-C=-$ -

$n-201836-05 / 26 / 74-14-22-10.5--0.6-175-$
$n-201837-05 / 26 / 79-14-20-16.12-0-7.6-670-$ 
LASL Uranium Hydrogeochemical and Stream Sediment Reconnaissance Data Listing

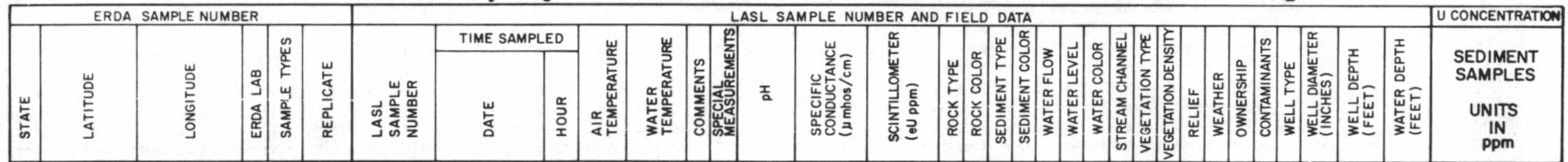

$56-44.7297-107.0672-2-12-$ $56-44.0963-104.7155-?-15-$ $56-44.0105-104.8194-?-15-$

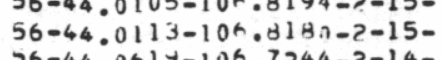
$56-44.0588-106.8077-2-15-$ $36-44.0583-106.8080-2-15-$ $36-44.0583-106.8087-2-15-$
$56-44.0777-10+.7794-2-15-$ $56-44.0866-106.80 ? 5-7-11-$ $36-44.0866-104.80 ? 5-?-11-$
$36-44.0738-1$ U4. $7>55-7-11=$ $36-44.0933-1$ Uf. $7736-2-15-$ $36-44.0933-106.7736-2-15-$
$56-44.1) 847-106.7075-2-15-$ $56-44.1) 847-106.7301-2-15-$
$56-44.1305-10 \% .7338-2-97=$ $56-44.2038-104.752 ?-7-14-$ $56-44.233(i-1) 4.7791-2-15-$ $56-44.2327-1$ Uस. $7525-?-12-$ $36-44,1597-10 \pi, 7075-c-12-$
$56-44,1783-106.7944-2-11-$ $56-44.1783-104.7944-?-11-$
$56-44.1691-1$ UA. $8077->-12-$ $56-44.1691-104.80 ? 7-?-12-$
$36-44.1877-106.8307-2-12-$ $36-44.1877-106.8307-2-12-$
$56-44.1569-106.91811-?-11-$ $56-44.1569-104.9180-?-11-$
$56-44,1425-1$ J4. $9169-2-12-$ $56-44.1425-1$ Jh. $9169-?-12-$
$56-44.150 n^{-106.9697-?-12-}$ $56-44.150 n-104.9697-?-12-$
$56-44.1508-104.9686-2-12-$ $56-44.1508-110 \mathrm{H} .9086-2-12-$
$36-44.1294-104.9388-2-11-$ $36-44.1274-104.9388-2-11-$
$56-44.131 y-104.90 r-10-211-$ $56-44$. $1263-1 \cup 4.9191-2-12-$ $56=44.11980-10 \% .9694-2-12-$ $\left.56-44.11 \forall_{11}\right)-1 u^{4} .9535-2-12-$ $56-44.0916-1$ uh. 9 /5?-2-12$36-44.075 n-104.9736-2-12-$ $56-44.1700-106.913 r,-2-12-$ $56-44,1797-10 \mathrm{~h} .9191-?-12-$ $56-44.2000-10 \mathrm{~h} .9413-2-12-$ $56-44,3675-10 \%, 7138->-12-$ $36-44.3861-10 \mathrm{~K}, 707>->-12-$ $56-44.3927-116.8177-2-12-$ $56-44.4100-1$ Uh. 81 inn-2-94$56-44.413 n-10 \%, 8655-?-12-$ $56-44.4383-10.6 .852>->-1<-$ $56-44.4275-11) 6.7861-2-12-$ $36-44.425 n-166.7886-2-1 \bar{c}-$ $56-44.4836-106.8027-?-12-$ $56-44,5233-106.738 n->-12-$ $56-44.5 ? 36-166.71 n ?-2-11-$
$56-44.5277-106.0872-2-99-$ $36-44.5219-106.6413-7-12$ $36-44.5883-10 f .668 n-7-12-$ $36-44.5738-10 t .716 h->-14-$
$36-44.0074-104.8013-2-12-$ $n-201839-05 / 26 / 74-15-21-14.11--0-7.4-575-$ $n-201841-05 />7 / 76-9-19-$
$n->01843-n 5 / 7>7 / 75-11-23-12.1:=-6.5-265-$ $n \rightarrow>01843-n 5 / 27 / 75-11-23-1201:-0.6 .5-265-$
$1) \rightarrow 01844-05 / 27 / 74-13-21-20$ $0=201946-n 5 / 37 / 7 n-13-26-23.0=-0.4-15 n 0$ $n=$. ח

n- $01948-95 / 27172-15-24-$

- -

$n 01851-05 / 27 / 7 h-16-22-18 . n-=-6.2-460$ a-70135P-n5/27/7h-16-24-14.n- - - G.h- 340$0-701953-n 5 / 27176-17-22-$

$n-201854-n 5 / 27 / 76-17-21$ -

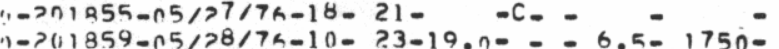
$-201801-n 5 / 28 / 7 n-10025-$ $1=>n 1862-n 5 />8 / 7 h-10-24-17,11-=6.9-1300-$ -201503-05/ $38 / 7 h-11-27-2 n \cdot 0--7.2-33000-$ $1=201866-05 / 28 / 7 n-13-21-7 . n-=-5 . n-50-50$ $n=201866-n 5 / 28 / 7 h-13-21-7 . n-=5 . h-150-$
$1 \rightarrow 01367-n 5 / 28 / 7 a-15-19-13 . n-=-5.7-18 n n-$

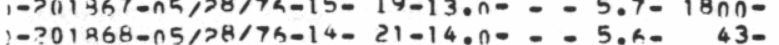

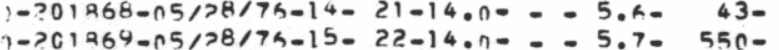

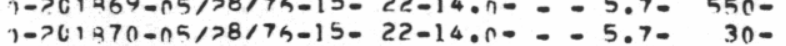
$n \rightarrow>01871-05 />8 / 7 A-15-21-8.0-=-5.7-600-$ $1->01873-05 / 38 / 7 h-16-21)-9.7--5.5-700-$ $\begin{array}{lll}1-201273-n 5 / 38 / 7 h-15-19-9 . n-2-5.6- & 44- \\ 11-201974-05 / 28 / 75-15-19-9.0- & -5.7-35-\end{array}$

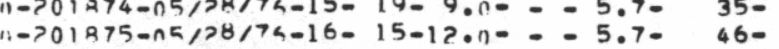
$n-201276-05 / 28 / 74-16-18-14 . n-0-5.9-56-$ $1->01877-n=/>8 / 74-16-18-14 \cdot 11--5.5-39-$ ->01978-nE/>8/7h-16-15-9.n-- - 5.7- 36- $201879-05 / 28 / 75=17-18-9.00-5.7-61-$ - >UाRRO-05/?38/7h-18- 15-8.11- - - 5.8- 550-

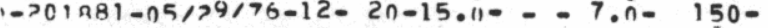
->01RR2-n5/>9/75-13- 20-15.01- - - 6.8- $1100-$ $n=>01883-05 />9 / 7 h-13-2 n-14 . n--0.7-31 n-$

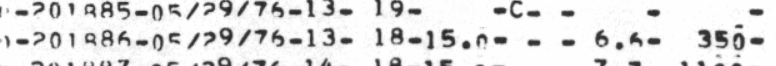
" $\Rightarrow 01887-n 5 />9 / 76-14-18-15.0=-7 \cdot 7-1100-$ $-701889-05 / 29 / 73-15-18-13.5--7 . n-650-$ - $>1890-n 5 />9 / 7 h-15-18-13.0=-5.8-100-$ - $>01891-n 5 />9 / 75-1 b-18-11,00-0-6.3-7 n 0-$ $\rightarrow 01294-05 / 31 / 74-11-26-20.0---7.2-1300-$

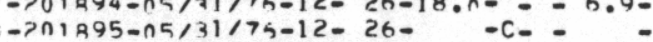
-?01R96-na/21/7h-13-2h-21..:- - 7.2- 3nño$\Rightarrow 01900-n 5 / 21 / 74-14-25-21 . n--0.9-1000-$ - $01001-05 / 31 / 75-14-25-19.0-=-6.9-7 n 0-$ 1) $\rightarrow 01902-05 / 31 / 74-14-25-15.0-=-6.8-8 n 0-$
$4-1-6-4-6-3-3-1-2-2-3-3-1--1--$ $12-1-6-6-6-1---4-3-2-1--1=$ $8-1-7-4-6----2-2-3-1--1-=$ $8-1-7-4-6----2-2-3-1--1-=$ $8-1-7-4-6--3-2--2-2-3-1=-1-=$
$15-1-6-5-6--3-2--3-2-3-1=-1=$ $4-1-7-5-6----2-4-3-1--1=$ $4-1-7-5-6----2-4-3-1=-1-=$ $14-1-7-5-6--2--4-2-3-1=-1-=$ $7-1-4-6-8-2-3-1-1-1-2-4-1--1=-$ $6-1-7-4-6-3-3-1-1-1-3-3-1--1-=$ 14-1-7-4-6- - - - -3-5-2-1--1- 2-1-6-4-6- - - -3-2-2-1- -1- $15-1-6-5-6----4-2-2-1--1=-$ $2-1-6-4-60-0=0-0-3-2-1=-1=-$ $0-1-6-4-6-2-3-1-2-4-3-3-1--1=$ $1<-1-6-4-6-2-5-1-3-3-3-3-1--1=$ $12-1-7-6-6-2-3-1-1-1-2-2-1--1-$ $11-1-4-4-4-4-4-1-2-2-3-4-2--1=$ 3-1-6-4-6-2-3-1-2-2-3-4-2- -1= $4-1-8-6-8-3-4-1-2-2-3-3-2--1-$ $11-2-7-5-8-4-4-1-2-1-4-5-1--1-$ $11-2-7-5-8-4-4-1-2-1-4-5-1--10$ $3-3-7-4-6-2-3-1-2-4-3-4-2--1=$ $4-3-7-2-$
$0-3-7-4-6-4-4-1-2-2-2-3-3-2-3-4-2--1$ $9-3-7-4-6-4-4-1-2-2-3-4-2--1-$
$6-1-6-4-6-3-4-1-2-2-3-3-3--1=$ $6-1-6-4-6-3-4-1-2-2-3-3-3--1$
$5-1-6-4-8-4-4-1-2-4-3-3-4-$ $5-1-6-4-8-4-4-1-2-4-3-3-4--1-$
$5-1-6-4-8-3-4-1-2-2-2-3-3--1-$ $5-3-7-5-8-4-4-1-2-1-2-3-4--1$ $4-1-6-4-6-4-4-1-2-3-3-4-4--1$
$3-2-8-4-6-4-4-1-2-1-4-5-3-1$ $3-2-8-4-6-4-4-1-2-1-4-5-3--1-$
$16-1-8-5-8-4-4-1-2-1-3-2-2--1-$ $16-1-8-5-8-4-4-1-2-1-3-2-2--1-$
$3-1-7-4-6-4-4-2-2-2-3-2-2-1-$ $3-1-7-4-6-4-4-2-2-2-3-2-2--1$
$15-1-7-6-6-4-4-1-2-2-3-2-2-$ $15-1-7-6-6-4-4-1-2-2-3-2-2--1$
$3-1-7-6-6-3-4-2-2-2-3-2-3--1$ $3-1-7-6-6-3-4-2-2-2-3-2-3-1-1-$ $20-1-7-4-6-3-4-2-2-2-3-2-4--1$ $12-1-7-5-6-3-3-1-2-2-3-2-4--1-$ $11-1-7-6-6-4-4-1-2-2-3-2-3--1-$ $12-1-7--4-4-1-2-2-3-3-3--1=$ $11-1-7-4-6-4-4-1-2-2-4-2-3-1-1=$ $8-1-7-5-6-2-2-1--4-3-3-2--1=$
$14-1-7-4-6-6---3-3-3-2--1=$ $11-1-7--0-2-3-1-1-3-3-2-2--1-$ $11-1-7-6-8-3-3-1-1-3-3-2-2--1-$ $7-1-7-6-8-1-3-1--4-3-2-2--1=$
$17-1-7-6-8-2-2-1-1-2-3-2-3--1=$
5,6

2.10

10

2.3

3.5

1.70

2.80

1.5

1.30

1.00

4.20

2.50

3.70

3.00

2.90

2.50

3.60

3.30

1.90

1.80

190

3.20

3.00

3.10

2.80

.50

.70

.70

2.80

3.00

10.30

2.00

.50

2.80

2.50

2.80

2.60

.90

$2.60^{\circ}$

2.50

2.10

2.50

2.10

3.00

3.60
2.70

2.40

10.30

2.70
3.00 
LASL Uranium Hydrogeochemical and Stream Sediment Reconnaissance Data Listing

$56-44.6102-104.798 n-?-12-$
$56-44.5858-104.795 n-2-11-$ $56-44.595+10+.8041-2-12=$ $56-44.5952-1$ uf. $8061-2-12$ $56-44.5572-106.9358-2-12-$ $56-44.5511-106.9008-7-12-$ $56-44.5788-1$ Uh. $9177-2-12-$ $56-44.5947-1$ of $87705-?-15-$
$56-44.5283-106.9463-2-12-$ $56-44.5313-10+.8466-2-12-$ $56-44.5291-106.8177-2-11-$ $56-44.530 n-106.8794-2-1 p-$ $56-44.4691-106.9136-7-12-$ $56-44.4697-10 K .9127-?-1$ k$56-44.4688-166.9025-2-11-$
$56-44.3194-10 \% .9311->-11-$ $56-44.3161-106.9405-2-11-$ $56-44.3072-10<.97,1,-2-11-$ 56 -44.305 $56-44.3025-106 \cdot 9739-2-1>-$ $56-44.30563-106,9003-3-12-$ $56-44.2566-106.915 n-2-12-$ $56-44.3525-106.8947-2-11-$ $56-44.3238-106.8838-7-11-$ $56-44.0738-105 \cdot 3474-2-14=$ $56-44.0972-10=0.3933-2-15-$ $56-44.1022-10=.4735-2-15-$ $56-44.0205-105 \cdot 2766-2-15-$ $56-44.0480-105.2833-2-15-$ $56-44.0327-105.352 ?-7-15-$ $56-44.0433-105.3211-2-15-$ $56-44.0961-105.2555-2-15-$ $56-44.0916-106.2277-2-15-$ $56-440297-10402111-8-150$ $56-44.0297-106.2111-2-15-$ $56-440125-1040430-7-15=$ $56-44.1125-100.2835-2-15-$ $56-44.1122-104.2036-?-15-$ $56-44.0911-106.371,7-2-97-$ $56-440950106.3477-209-150$ $56-44.0925-10 k \cdot 3194-2-15-$ $56.44 .07310-104.2620-2-15=$ $56.44 .073 n-104.2623-2-15$ $56.44 .063110 \% .2635-2-15-$ $56.44 .05307-10+.3333-2-15=$ $56-44.0275-10 \% .3333-2-15=$ $56.44 .0100-104.3641-2-97$ $n=201904-05 / 31 / 7 n-15-25-15 \cdot n--7 \cdot 1-775-$ $->01906-15 / 31 / 74-16-24-11 \cdot n-=-6.9-650-$
$-201907-05 / 31 / 76-16-24-17.0-=-7.7-900-$ $\rightarrow 01908-05 / 31 / 75-16-23-17.0-0-6.5-1100-$ (1-2019n9-0k/ri1/7a- 8- 18-7.11- - - 5.5- 52$1=>01910-n k / n 1 / 7 h-9-2 n-8.6=-5.8-6 n 0-$ $\rightarrow 001911-0 k / i 1 / 7 a-10-20-8.0=-5.3-34-$

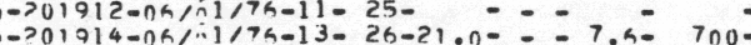
$->01915-n a / n 1 / 7 a-13-27-17 \cdot 0=-6.3-165-$ $\Leftrightarrow \rightarrow 01917-0 \times / 0.1 / 76-14-28-13.5--0.9-730=$ $-201918-n G / C 1 / 7 h-14-28-$

$-201919-0 h / n 1 / 7 h-15-24-11 . n=-5.6-500-$ $-201021-0 k / 01 / 7 k-15-24-900-=-600=$

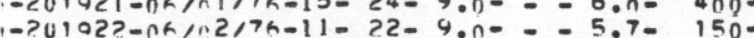
作 (15 $-201925-n 6 / 72 / 75-13-23-\quad-\cdots-5=$

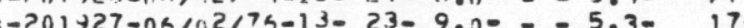
-201928-n6/i2/7h-13-21-10.01)- - 5.5- 6ño-

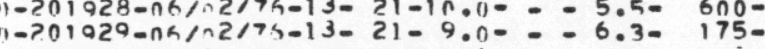
$n-20173 n=06 / i 2 / 7 n-13-20-A_{0}, i=-6.3-180-$ - $201935-07 / 20 / 7 h-15-30-26 . n-$ - - 6.3- 2000 - ?01936-07/20/7h-15- 30- $0194 ?-07 / 20 / 76-17-3 n-$ $->01945-07 / 21 / 76-11-30-$
$-701946-07 / 21 / 7 h-11-30-$ (1) - > $01947-n 7>2176-13-31-$ $->01951-1 n / 10 / 75-16-18-$ $-201956-1 n / 10 / 76-17-17=$

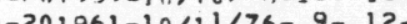
- $01961-1$ i -201963-1n/11/7h-10- 13 (140 $\rightarrow 01966-1 n / 11 / 79-10-14-$ 1n/11/76-11- 16 $->01070-1 n / 11746-11-16-$ ?01972-1n $\rightarrow$ - $1976-1 n / 11 / 7 a-12-16-$ $n-? 01977-1 n / 1174-13-16-$
$n=001978-1 n / 1174-13-17-$
$17-1-7-6-6-2-3-1-1-4-3-2-3--1-=$ $16-1-4-5-6-2-3-1-1-2-2-2-3--1=$
$9-1-4---2-3-1-1-3-3-2-3-$ $12-1-4-5-6-2-2-5-3-3-3-2-3--1-$ $6-1-7-5-6-4-4-1-2-1-4-3-1=-10$
$3-1-7-4-6-4-4-1-2-2-4-3-1--1-$ $3-1-7-4-6-4-4-1-2-2-4-3-1--1-$
$6-1-7-6-6-4-4-1-2-2-3-2-2--1=$ $14-1-7-5-8-1--2-4-3-2--1=$
$21-1-7-6-8-1-2-5-1-4-3-2-2--1=$ $75-1-7-6-6-3-4-1-2-2-3-2-2--1-$ $4-1-7-6-8-3-3-1-3-3-3-2-2--1-$ 1-1-7-5-6- - - -3-3-2-2- -1$6-1-7-4-6-4-3-1-2-4-3-3-2--1=-$
$8-1-7-4-6-4-3-1-2-4-3-3-2--1-$ $6-1-7-4-6-2-2-1-1-4-3-3-2--1=-$
$6-1-7-4-6-2-3-1-2-2-3-4-2-1-$ $4-1-6-6-8-2-3-1-2-2-3-3-2--1-$ $12-1-6-5-6-2-2-1-3-4-3-3-2--1=$ $2-3-4-4-6-4-4--1-4-4-2--1=-$
$8-3-4-4-6-4-4-1-2-1-4-4-2-1=$ 6-1-6-4-6-4-4-1-2-1-4-3-2- -1$5-4-6-4-6-3-4-1-2-3-4-2-3--1-$
$4-3-7-5-8-2-2-1-2-4-3-4-4--1-$ 2-1-7-6-6-2-3-1-2-4-3-4-3- -1- -

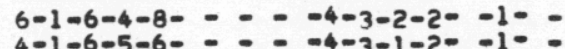
$3-1-6-5-6-\ldots-04-3-2-2--1-$ $1-2-1-5-6-0--4-3-1-1--1=-$ $6-1-4-5-6-2=-4-3-2-1--1=$ $6-1-7-5-6-1-0-4-3-1-1--1-$
$3-1-7-5-6-$ $4-1-6-4-7--0-4-3-3-1--1-=$ $16-1-7-4-6--1--2-4-3-2-1=-1-=$ $-1=$ $16-1-7-4-6--1=-2-4-3-2-1=-1=$ $16-1-7-4-6-0--2-4-3-2-1--10=$ $16-1-7-4-6--1--2-4-3-2-1--1-=$ $17-1-7-4-6--1--3-4-3-2-1--1-$ $17-1-7-4-6--1--3-4-3-2-1-1-1=$
$16-1-7-4-6--1--3-4-3-2-1--1=$ $17-1-7-4-6--1--2-4-3-2-1--1=$ $16-1-7-4-6--1--2-4-3-2-1=-1-$ $14-1-7-4-6----2-4-3-2-1--1=$ $16-1-7-4-6--1=-2-4-3-2-1=-1-=$ $19-1-7-4-6--1--2-4-3-2-1--1-=$ $16-1-7-4-6--1--2-4-3-2-1=-1-=$ $17-1-7-4-6--1--2-4-3-2-1=-1-=$ $16-1-7-4-6--1--2-4-3-2-1--1=$

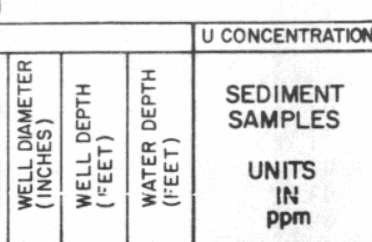

$\underset{\substack{\text { IN } \\ \text { Ppm }}}{\text { ITS }}$

.40

2.80

3.00

4.10

2.40

3.40

3.60

1.70
3.10

2.50

2.10

2.30

3.00

3.80

2.70

2.20

.50

.70

2.50

1.50
2.40

.30

.30

3.00
3.50

2.50

5.80

3.80
3.20

.20

3.00

3.00

.50
3.80

3.80
3.60

60

3.40
.60

5.60

3.00

3.90

3.90

3.80

(3)

3.30
3.70

89

3.00

5.40 


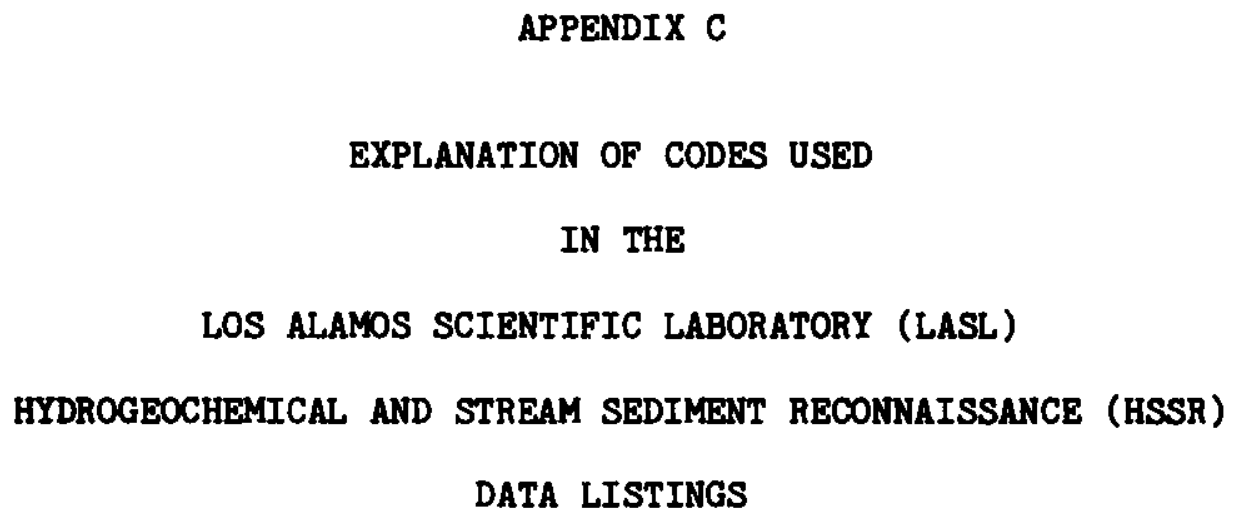




\section{APPENDIX ITEM C-I}

EXPLANATION OF CODE USED

IN OPEN-FILE LISTINGS OF

HYDROGEOCHEMICAL, AND STREAM SEDIMENT RECONNAISSANCE DATA

PROVIDED BY

THE LOS ALAMOS SCIENTIFIC LABORATORY

\section{ERDA SAMPLE NUMBER}

STATE: A two-digit Federal Information Processing Standards (FIPS) code, designating the state from which each sample came. For the states being covered by the LASL, the code numbers are:

$$
\begin{aligned}
& \text { Alaska }=02 \\
& \text { Colorado }=08 \\
& \text { Montana }=30
\end{aligned}
$$

$$
\begin{aligned}
& \text { New Mexico }=35 \\
& \text { Wyoming }=56
\end{aligned}
$$

LATITUDE AND LONGITUDE: Sample location, in degrees and decimal degrees to four places. However, though generally much better, locational accuracy cannot be guaranteed closer than about $300 \mathrm{~m}(1000 \mathrm{ft})$.

ERDA LAB: An Energy Research and Development Administration (ERDA) one-digit identifier designating the national laboratory responsible for taking the sample and the data shown in the listing, as well as providing the analysis giving the uranium and other elemental concentrations, if any. The LASL is designated by the numeral 2 .

SAMPLE TYPE: A two-digit identifier which specifically designates the pertinent properties defining the sample type to which the listed data relate. For explanation of the code used, refer to the attached "Numerical Key and Specifications for Sample Types Taken by the LASL."

REPLICATE: A three-digit sequential number assigned to indicate a multiple sample of a single sample type from a single location. The largest number in use indicates the most recent sample taken, and there will always be smaller sequential numbers representing earlier samples back to 000 , which is the initial sample from any given location. Except in the case of special studies, there will be no replicate samples and this entry will therefore be 000 .

\section{LASL SAMPLE NUMBER AND FIELD DATA}

LASL SAMPLE NUMBER: A unique six-digit number permanently assigned by the LASL to every location sampled in each state. For internal use, these numbers are assigned in blocks to the various areas individually treated and reported upon, and therefore serve to generally locate the samples within the various states as follows.

Location Numbers

from 000001 through 099999

from 100001 through 199999

from 200001 through 299999

from 300001 through 399999

from
State

$=$ New Mexico

= Colorado

$=$ Wyoming

$=$ Montana

= Alaska 
TIME SAMPLED: The DATE that the sample was taken, in terms of the number of the MONTH, followed by the DAY and finally the YEAR, separated by slashes, and then the TIME it was taken on that data to the nearest whole HOUR on a military (24-hour) clock.

AIR TEMPERATURE: The temperature that was measured in the shade at the time of sampling, to the nearest whole degree Celsius $\left({ }^{\circ} \mathrm{C}\right)$.

WATER TEMPERATURE: The temperature that was measured in the sample water (in situ whenever possible) at the time of sampling, to the nearest one-tenth of a degree Celsius $\left(0.1^{\circ} \mathrm{C}\right)$.

COMENTS: A "C" in this column indicates that some secondary comment not included in the listing was recorded at the sample location. This information will be used by the LASL in evaluating the data and, if appropriate, it will be mentioned in the final report.

SPECIAL MEASUREMENTS: An "S" in this column indicates that one or more field measurements in addition to those listed were made at the sample location. A description of any special parameters measured and the measured value at each sample location will be included in the final HSSR survey report on the area.

$\mathrm{pH}$ : The $\mathrm{pH}$, to the nearest one-tenth $(0.1)$ of a $\mathrm{pH}$ unit, that was measured in the water at the sample location at the time of sampling.

SPECIFIC CONDUCTANCE: The conductivity, in $\mu \mathrm{mhos} / \mathrm{cm}$, that was measured in the water at the sample location at the time of sampling.

SCINTILLOMETER: The equivalent uranium (eU), in parts per mililion (ppm), as measured on a flat ground surface within $10 \mathrm{~m}$ of the sample location using a scintillometer fitted with a differential gamma sampler (DGS). The effect of the DGS is to introduce a fixed geometry into the measurement and remove the background.

ROCK TYPE: The single digit in this column provides a general description of the dominant lithologic regime at or near the sample location, as given below.
1 = Sedimentary
2 = Metamorphic
$3=$ Igneous
4 = Unknown

ROCK COLOR: The single digit in this column provides an indication of the observed dominant color of local bedrock exposures at or near the sample location, as given below.

$$
\begin{array}{lll}
1=\text { White/Buff } & 4=\text { Pink } / \text { Red } & 7=\text { Gray } \\
2=\text { Yellow } & 5=\text { Green } & 8=\text { Black } \\
3=\text { Orange } & 6=\text { Brown } & 9=\text { Other }
\end{array}
$$

SEDIMENT TYPE: The single digit in this column provides a subjective evaluation of the dominant sediment type at the sample location, as given below.
$1=$ Boulders
2 = Cobbles
4 = Sand
3 = Gravel
$5=$ Mud
$6=$ Muck

7 = Other 
SEDIMENT COLOR: The single digit in this column indicates the observed dominant color of the bottom sediment (stream channel, lake bed, etc.) at the sample location at the time of sampling, as given below.
$I=$ White/Buff
4 = Pink/Red
7 = Gray
$2=$ Yellow
5 = Green
8 = Black
3 = Orange
6 = Brown
9 = Other

WATER FLOW: The single digit in this column provides a subjective evaluation of the wter movement at the sample location at the time of sampling, as given below.
1 = Stagnant
2 = Slow
$3=$ Moderate
5 = Torrent
4 = Fast

WATER LEVEL: The single digit in this column provides a subjective estimate of water quantity at the time of sampling, relative to its usual condition at the sample location, as given below.
$1=$ Dry
$3=$ Normal
5 Flood
$2=$ Low
$4=$ High

WATER COLOR: The single digit in this column provides a subjective evaluation of suspended load in the sample water as, given below.
$1=$ Clear
3 = Cloudy
$5=$ Algal
$2=$ Murky
4 = Muddy
6 = other

STREAM CHANNEL: The single digit here gives a subjective evaluation of stream channel character at the sample location at the time of sampling, as given below.

$$
1 \text { = Depositing } 2 \text { = Eroding } \quad 3 \text { = Unknown }
$$

VEGETATION TYPE: The single digit in this column provides a subjective evaluation of the dominant plant type in the vicinity of the sample location, according to the key below.
$1=$ Conifers
$4=$ Grass
7 = other
2 = Deciduous
$5=$ Moss
3 = Brush
$6=$ Marsh

VEGETATION DENSITY: The single digit in this column provides a subjective estimate of the amount of plant cover in the vicinity of the sample location, according to the key below.
1 = Barren
$3=$ Moderate
5 = Very Dense
2 = Sparse
4 = Dense

RELIEF: The single digit in this column provides a subjective evaluation of the topography within a few hundred meters of the sample location, according to the key below.
$1=$ Flat
$2=\operatorname{Low}(<15 \mathrm{~m})$
$3=$ Gentle $(15-60 \mathrm{~m}) \quad 5=\operatorname{High}(>300 \mathrm{~m})$
4 = Moderate $(60-300 \mathrm{~m}) 6$ - Other 
WEATHER: The single digit in this column gives the observed climatic condition at the sample location at the time of sampling, as given by the key below.
$1=$ Clear
2 = Partly cloudy
$3=$ Overcast
$5=$ Snowy
$6=$ other

OWNERSHIP: The single digit here gives a broad classification of administrative responsibility or general ownership of the land at the sample location, according to the key below.
1 = Federal
3 = Private
$5=$ other
2 = State
4 = Indian

CONTAMINANTS: The single digit here indicates known or suspected local factors likely to influence analytical results, according to the key below.
$1=$ None
2 = Mining
3 = Agriculture
4 = Industry
5 = Sewage
6 = Power generation
$7=$ Urban
8 = Recreation
$9=$ Other

WELL TYPE: The single digit in this column provides a general description of the type of water well from which the sample was taken (1f, in fact, it was a well sample), according to the key below.
1 = Windmill-stock
4 = Suction pump
7 = Hand bail
2 = Windmill-domestic
5 = Jet pump
3 = Submersible pump
6 = Large turbine
8 - Unknown
$9=$ Other

WELL DIAMETER: The one or two digits (if any) in this column give the measured or estimated inside diameter, in inches, of the casing of the well from which the sample (if taken from a well) came.

WELL DEPTH: The one, two, or three digits (if any) in this column give the total drilled depth from the surface, in feet, of the well from which the sample (if taken from a well) came. Three $9 \mathrm{~s}$ in this column indicates a well. depth greater than $1000 \mathrm{ft}$.

WATER DEPTH: The one, two, or three digits in this column give the depth, in feet, from the surface to the standing water in the well, if known (if the sample was taken from a well). A -1 in this column indicates a flowing artesian well.

URANIUM CONCENTRATION: The value given in this column is the analytically derived value of the uranium concentration found in the water sample in parts per billion (ppb), or in the sediment sample in parts per million (ppm). Sample Types No. 1 through 10 and 21 through 30 are water samples, with their uranium concentrations given in ppb, while Sample Types No. 11 through 20 and 31 through 40 are sediment samples, with their uranium concentration given in ppm. 


\section{APPENDIX ITEM C-II \\ ABBREVIATED NUMERICAL KEY AND SPECIFICATIONS FOR COMMON SAMPLE TYPES TAKEN BY \\ THE LOS ALAMOS SCIENTIFIC LABORATORY (LASL) \\ IN THE NATIONAL URANIUM RESOURCE EVALUATION (NURE) \\ HYDROGEOCHEMICAL AND STREAM SEDIMENT RECONNAISSANCE (HSSR)}

This numerical key provides the necessary tie between the specific type or form of each sample taken in the LASL HSSR program and each individual suite of field and laboratory data to which the sample relates. It defines the various sample types generally to be collected by the LASL in the ERDA HSSR for uranium.

The two-digit key number assigned to each sample type herein designates three distinct properties of the most common types of samples taken by the LASL in the NURE HSSR project. These properties are: (a) The general sample source (spring or stream or dry stream, etc.); (b) The sample medium (water or sediment, etc.); and (c) The treatment given the sample in the field or laboratory prior to its analysis by the LASL.

The key numbers are inserted in the appropriate columns of the specially formatted ERDA sample numbering system to positively identify the sample type for all LASL sample data submitted. An expanded version of this key, which includes sample types less commonly used the the LASL, will be provided whenever data from such samples are submitted.

\section{KEY NO. $\quad$ SOURCE / MEDIUM / TREATMENT}

01 - Spring water sample untreated.

02 - Stream water sample untreated.

03 - Well water sample untreated.

04 - Natural pond water sample untreated.

05 - Artificial pond water sample untreated.

06 - Spring water sample filtered through an 0.45- membrane filter and acidified to a $\mathrm{pH}$ of $\leq 1$ with reagent-grade nitric acid ( $\mathrm{HNO}_{3}$ ).

07 - Stream water sample filtered through an $0.45-\mu$ membrane filter and acidified to a $\mathrm{pH}$ of $\leq 1$ with reagent-grade nitric acid ( $\mathrm{HNO}_{3}$ ).

08 - Well water sample filtered through an $0.45-\mu$ membrane filter and acidified to a $\mathrm{pH}$ of $\leq 1$ with reagent-grade nitric acid ( $\mathrm{HNO}_{3}$ ).

09 - Natural pond water sample filtered through an $0.45-\mu$ membrane filter and acidifled to a $\mathrm{pH}$ of $\leq 1$ with reagent-grade nitric acid ( $\mathrm{HNO}_{3}$ ).

10 - Artificial pond water sample filtered through an $0.45-\mu$ membrane filter and acidified to a $\mathrm{pH}$ of $\leq 1$ with reagent-grade nitric acid ( $\mathrm{HNO}_{3}$ ). 
11 - Wet spring sediment sample dried at $\leq 100^{\circ} \mathrm{C}$ and sieved to -100 mesh through stainless steel sieves.

12 - Wet stream sediment sample dried at $\leq 100^{\circ} \mathrm{C}$ and sieved to -100 mesh through stainless steel sieves.

13 - Wet natural pond sediment sample dried at $\leq 100^{\circ} \mathrm{C}$ and sieved to -100 mesh through stainless steel sieves.

14 - Wet artificial pond sediment sample dried at $\leq 100^{\circ} \mathrm{C}$ and sieved to $=100$ mesh through stainless steel sieves.

15 - Dry stream sediment sample dried at $\leq 100^{\circ} \mathrm{C}$ (if necessary) and sieved to -100 mesh through stainless steel sieves.

96 - Dry natural pond sediment sample dried at $\leq 100^{\circ} \mathrm{C}$ (if necessary) and sieved to -100 mesh through stainless steel sieves.

97 - Dry artificial pond sediment sample dried at $\leq 100^{\circ} \mathrm{C}$ (if necessary) and sieved to -100 mesh through stainless steel sieves.

98 - Other water

These key numbers are to be used only for water (98) or

99 - Other sediment sediment (99) samples coming from a special source and/ or given a special treatment not described for any of the types of samples above. When used in the listings published herein, the source and treatment given the samples so designated are described in the text. 


\author{
APPENDIX D \\ SUMMARY OF STANDARD PROCEDURES \\ USED BY \\ THE LOS ALAMOS SCIENTIFIC LABORATORY (LASL) \\ IN THE \\ US ENERGY RESEARCH AND DEVELOPMENT ADMINISTRATION (ERDA) \\ NATIONAL URANIUM RESOURCE EVALUATION (NURE) \\ HYDROGEOCHEMICAL AND STREAM SEDIMENT RECONNAISSANCE (HSSR)
}




SUMMARY OF STANDARD PROCEDURES
USED BY
THE LOS ALAMOS SCIENTIFIC LABORATORY (LASL)
IN THE
US ENERGY RESEARCH AND DEVELOPMENT ADMINISTRATION (ERDA)
NATIONAL URANIUM RESOURCE EVALUATION (NURE)
HYDROGEOCHEMICAL AND STREAM SEDIMENT RECONNAISSANCE (HSSR)

\section{FIELD PROCEDURES}

Water Sampling

Water samples are taken directly from the source wherever possible, filtered through a $0.45-\mu$ membrane filter into one each, prewashed and sealed, 4l-ml reactor "rabbit" and 25-ml vial (both polyethylene), and both are then acidified to a $\mathrm{pH} \leq 1$ with $8 \mathrm{~N}$, reagent.-grade, $\mathrm{HNO}_{3} \cdot$ All sample containers are doubly labeled with preprinted, adhesive labels carrying the same sample location number preprinted on the field data form. Springs are sampled as near to their point of emergence as possible; stream waters are taken from fast-flowing current away from the bank; ponds (including small lakes and reservoirs) are sampled from just below the surface, away from the bank; and well waters are taken near the wellhead if the well is pumping or from a holding tank if not.

\section{Sediment Sampling (Wet or Dry)}

Enough fine-grained, organic-rich, water-transported sediment to yield a composite sample of $25 \mathrm{~g}$ after processing (as indicated below) is taken from beneath the water level (where water exists) at three closely adjacent spots at each location. This is done with a polyethylene scoop, after the water sample (if any) is taken. The sediment is put into a new, clean, and originally sealed, rip-top polyethylene bag and properly double-labeled for delivery (with the field data form) to the contractor's drying facility. After drying at $\leq 100^{\circ} \mathrm{C}$, each sample is sieved through stainless steel sleves to -100 mesh. The -100 mesh fraction is put into a prewashed, 25-ml polyethylene vial, appropriately double-labeled (using labels from the data form), and sealed for shipment to the LASL.

\section{Field Measurements}

The air temperature, taken in the shade at the time of sampling, is recorded to the nearest whole degree centigrade. The water temperature is measured in the source water and recorded to the nearest one-half degree centigrade. All temperature measurements are made with quality, precalibrated thermometers. The $\mathrm{pH}$ of the source water is measured with a calibrated, portable $\mathrm{pH}$ meter or multi-range $\mathrm{pH}$ paper, and recorded to the nearest one-tenth of a $\mathrm{pH}$ unit. The specific conductance $(\mu \mathrm{mho} / \mathrm{cm})$ of the 30 urce water is measured with a calibrated, temperature compensated $\left(25^{\circ} \mathrm{C}\right)$ portable meter after the attached sample cup has first been rinsed three times in the source water. The scintillometer readings, taken on a flat, dry spot within a few meters of the sample location, are measured with a portable scintillometer. Two readings are recorded, the first with a radiation shield in place (blocking out ground radiation), and the second with the shield removed. The readings (in counts/s) are converted by computer to give the equivalent uranium ( $\mathrm{eU}$ ) value set forth in the data listing. 


\section{Field Observations}

These represent the best subjective judgment of the field sampler on location, and include very general descriptions of the local bedrock, sediment, water, vegetation, terrain, weather, possible contaminants, and water well configuration, if applicable.

Sample Location Verification

Each contractor is supplied field maps with the desired sample types and locations symbolically premarked at the LASL. The maps are normally USGS Quadrangles (either $7.5^{\prime}$ or $15^{\prime}$ ), but where not available, Forest Service, State Highway, or other reasonably detailed maps are provided. As each location is sampled, a unique sample location number, preprinted on transparent adhesive labels provided with the identically numbered field data forms, is pasted over the precisely marked site on the field map. The latitude and longitude of each location is computed by the sampling contractor. Every location is later checked (and corrected if necessary) at the LASL by overlaying computer produced location plots on the field maps used. The latitudes and/or longitudes are corrected if the overlay locations are displaced by more than $300 \mathrm{~m}$ from the locations marked on the field maps. When a desired location cannot be sampled as specified, an alternate sample type or location as near as possible to the original one is picked, and the new sample type and/or location is/are marked on the field map and properly labeled as above.

\section{ANALYTICAL PROCEDURES}

\section{Water Samples Analyzed for Uranium by Fluorometry}

In a controlled laboratory environment, a NaF (98\%)-LiF (2\%) flux pellet is prepared and placed on a platinum dish. The 25-ml water vial is vigorously shaken and a $0.20-\mathrm{ml}$ aliquot of water is withdrawn and dropped onto a flux pellet, then evaporated under a heat lamp. The sample flux is then heated until fused. After it cools, it is excited with ultra-violet radiation in the fluorometer, and the measured fluorescence is read, recorded, and put through a computer routine using standards and blanks run at the same time to obtain the uranium concentration. Water samples which have uranium concentrations in excess of $10 \mathrm{ppb}$ (the upper limit of detection of the fluorometry system without recalibration) are reanalyzed using the delayed neutron counting technique described below. Water samples which have uranium concentrations below $0.2 \mathrm{ppb}$ are concentrated by a factor of 10 using evaporative methods and are then analyzed by fluorometry to a lower limit of $0.02 \mathrm{ppb}$.

Water Samples Analyzed for Uranium by Delayed Neutron Counting (DNC)

Only waters with $>10 \mathrm{ppb}$ uranium are assayed using DNC. Samples taken in the 4l-ml rabbits are thoroughly cleaned (exterior) before analysis. Samples received in 25-ml vials (used exclusively in some of the early work) are transferred to clean, labeled, $41-\mathrm{ml}$ rabbits before being analyzed. Each water sample is weighed, and its weight (less that of the rabbit) and location number are recorded. The rabbits are then loaded into a 25-sample transfer clip. The reactor pneumatic transfer system and background radiation levels are checked, and the system is calibrated using four standards. The transfer clip is installed on the pneumatic feed line, and the count control is set 
(typically, a 60-s irradiation, a 30-s delay, and a 60-s count is used, but this can be changed to accommodate abnormally high or low uranium concentrations). The samples are cycled through the system and the uranium concentration is automatically measured, computed in $\mathrm{ppb}$, and entered into the data base.

Uranium Analysis of Sediment Samples

All sediment samples are analyzed for total uranium by DNC. A split of each sample (dried and sieved as described) is transferred to a clean 4-ml rabbit, weighed (less the tare), and recorded along with the appropriate location number. The readied rabbits are loaded into a 50-sample transfer clip. The reactor pneumatic transfer system and background radiation levels are checked, and the system is calibrated as above. The transfer clip is installed and the count control is set (typically, a 20-s irradiation, a 10-s delay, and a 20-s count is used). The samples are cycled through the system and the uranium concentration is automatically measured, computed in ppm, and entered into the data base. 


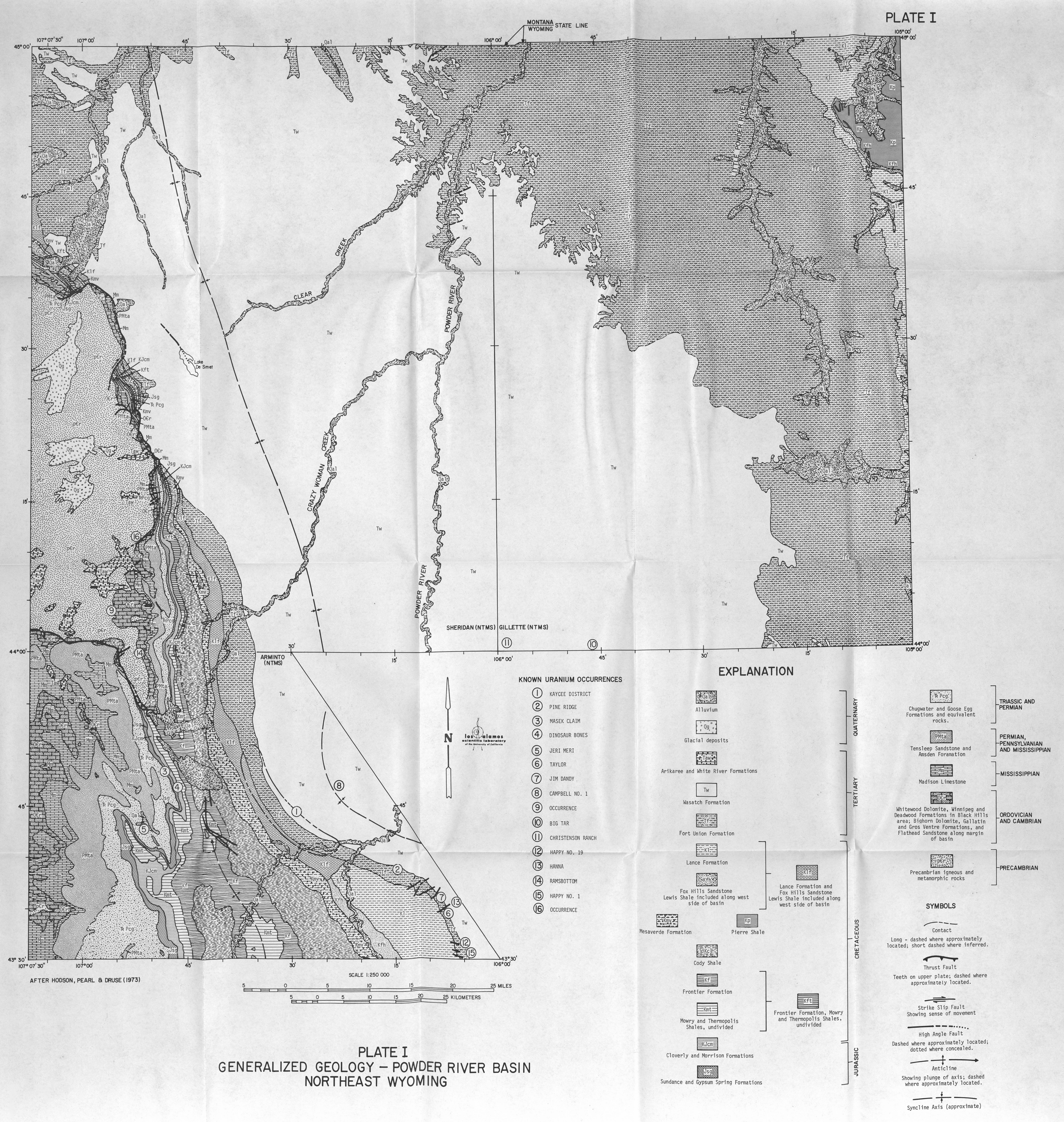





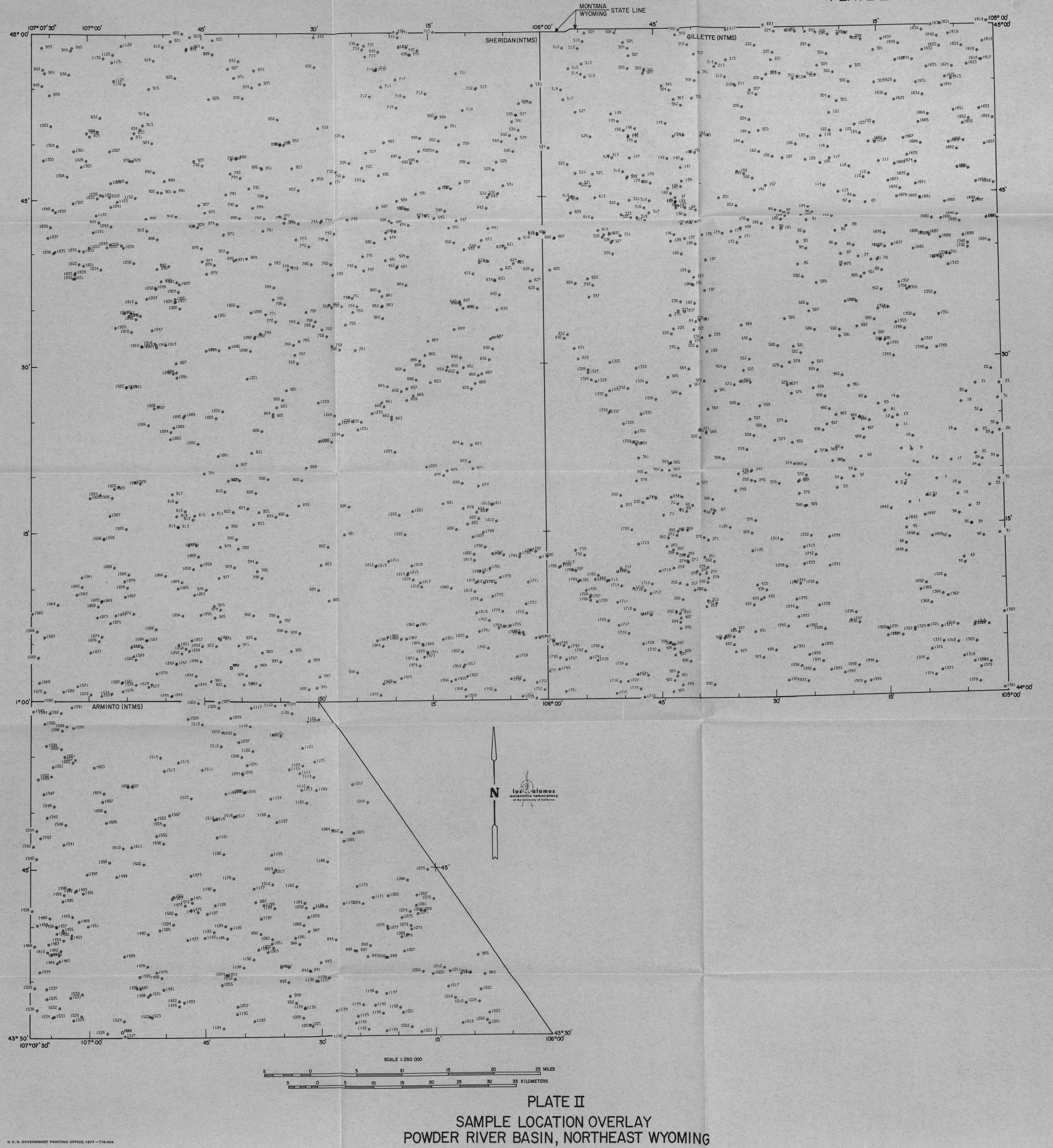



PLATE III
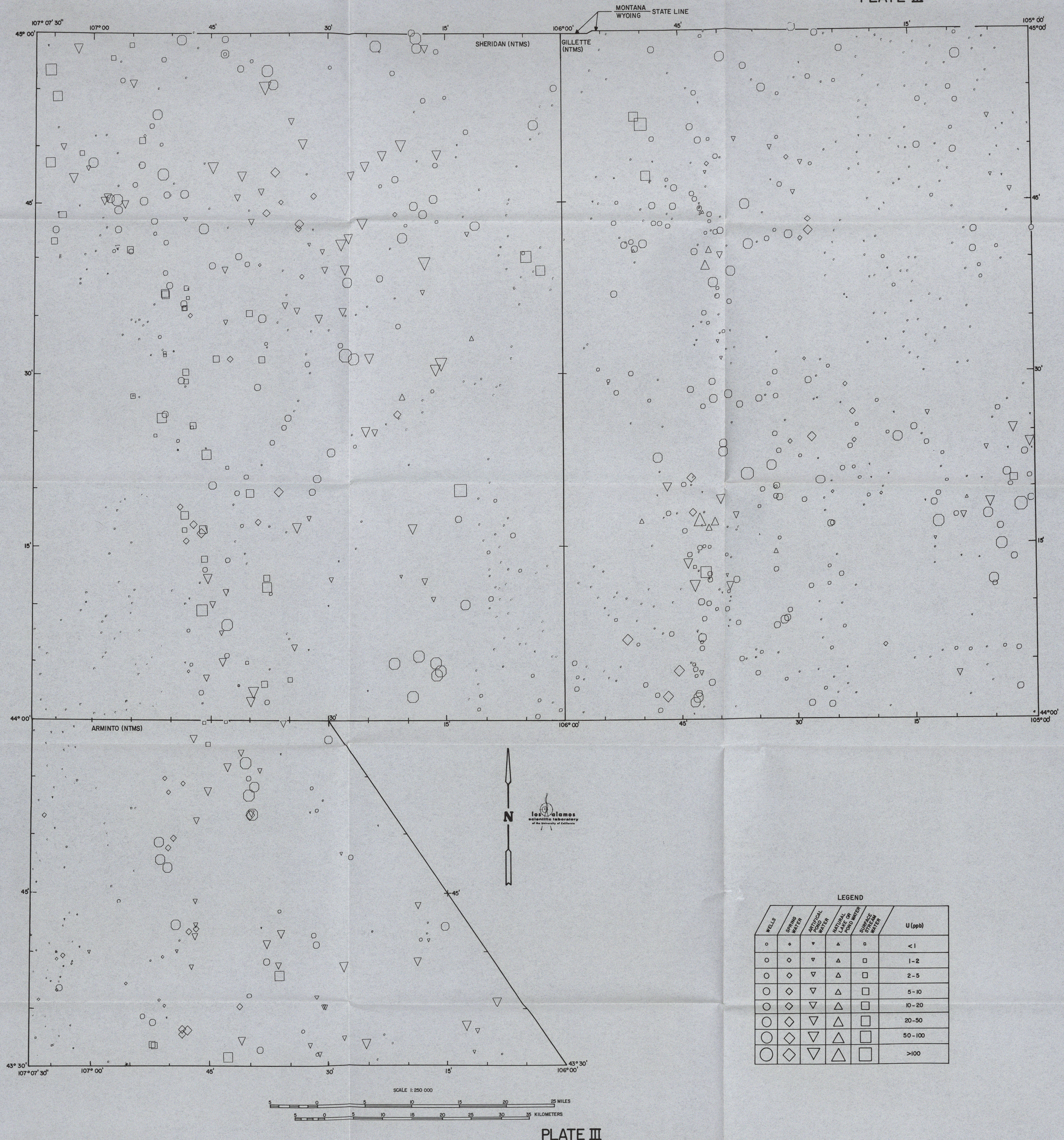

URANIUM CONCENTRATION OVERLAY FOR WATER (ppd) POWDER RIVER BASIN, NORTHEAST WYOMING 



